\title{
The Role of the Atlantic Corridor Project as a Form of Strategic Community of Practice in Facilitating Business Transformations in Latin America
}

A thesis submitted in partial fulfillment of the requirements

for the Degree of Doctor of Project Management

By

Alejandro C. Arroyo

MBA in Marine Resource Management, and MSc in Maritime Economics and Operations

School of Property, Construction and Project Management

Royal Melbourne Institute Technology University

May 2009 


\section{Certification}

I, Alejandro C. Arroyo, do hereby certify for this thesis that:

a. Except where due acknowledgement has been made, the work is that of myself alone,

b. The work has not been submitted previously, in whole or in part, to qualify for any other academic award;

c. The content of the thesis is the result of work which has been carried out since the official commencement date of the approved research program, and

d. That any editorial work, paid or unpaid, carried out by a third party is acknowledged.

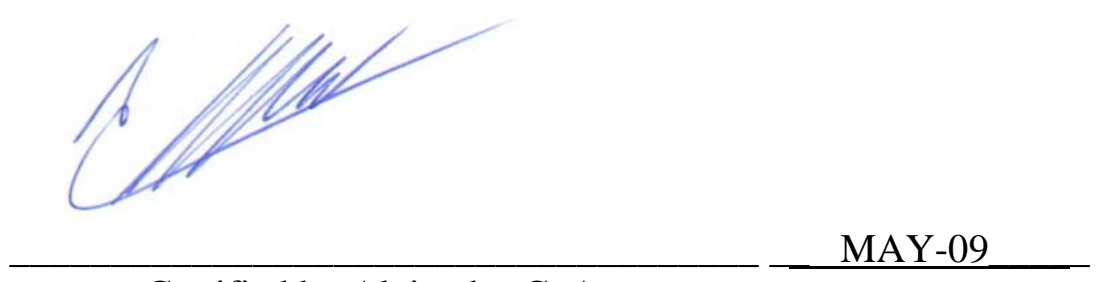

Certified by Alejandro C. Arroyo 


\title{
Acknowledgements
}

I would like to acknowledge the following people without whom this work could not have been completed.

\author{
Dr. Derek Walker
}

for being my mentor, coach, and guide throughout the doctorate program and for his comprehension and permanent encouragement to complete this work

\section{Silvina Henriksen Arroyo}

for being my confidant, sounding board, and anchor and, for helping me overcome countless difficulties along this long journey 


\begin{abstract}
This thesis examines the way that a change management entity facilitated a deep and lasting business transformation, undertaken as a major program of projects that were engaged in business process re-engineering, logistics supply change management and formation of joint ventures and forms of alliance. It takes a project management perspective of this change agent with a strong focus on how it achieved change through leadership, co-learning strategies and collaboration. This study takes place within the context of a unique historical period of economic transition for Latin America.

By the time the globalization wave started to hit South America in the late 80s, both its national economies and various logistic segments were used to operating within a protective and restrictive business context. Regional companies enjoyed operating within bilateral treaties and under the scope of a wide array of subsidies and protective regulations that let them grow without having to face the complexities of a truly competitive free market. On the contrary, foreign companies either found regional entry barriers simply too high or that these barriers deterred them from expanding across a new potential market.
\end{abstract}

During the early 90s, when the globalization phenomena combined with an economic opening process across the region, South America faced a deep business transformation process expanding along its coasts. The great South American common market known as Mercosur, began to experience a potential threat on the part of the global (and therefore more resourceful) world-class shipping companies, port and logistic operators who considered this region of the world as their next necessary step toward achieving larger economies of scale to help them remain globally competitive. South America in general, and the Mercosur market in particular, became strategic markets that these global firms could not overlook without running the risk of losing their global competitive advantage.

The globalization wave brought about two trends that forms a critical context to the essence of this research into the Mercosur Atlantic Corridor Project (the Corridor). These were: 
1. The opening process of the Mercosur economies toward a less regulated and a more liberal concept of trade which originated within a window of opportunity for the global operators to target the region; and

2. The national and regional operators' relative competitive weakness and their firm perception on the way the globalization wave might impact their business. This led to a remarkable regional business transformation process.

The Corridor was established as a regional reaction to the global threat by an increasing number of national and regional companies (both logistic and logistic-related firms) that decided to join forces to face (by then) an uncertain and threatening business environment. These operators came from a wide array of sectors within the shipping and logistic world: ocean and river transportation companies; marine and river port operators; air-station operators; trucking and railway companies; warehousing and distribution operators; freight-forwarding firms; air-cargo freighting operators; consultancy and surveying services; law and insurance services; exporting and importing companies; ship-building and naval repair firms; traders and brokers; etc.

The Corridor provides an example of a business transformation project involving extensive knowledge transfer and trust building as core elements and that identifying this through the research was a key outcome. The research examines the Corridor's ability to generate trust first and then to facilitate knowledge transfer among stakeholders. Not less relevant is the identification of leadership, collaboration, culture, and corporate strategy as vital conductors for stakeholders to share critical internal information and join forces to face extra-regional threats.

The research focuses on the Corridor as a single entity using five case studies of different complexity by exposing distinct angles of business transformation through the participation of a rather wide sample of companies and segments.

The case studies provide readers with the necessary elements to clearly visualize the extent reached by the globalization process across the region as well as help them identifying the views, opinions, and insights of all those who participated in a series of interviews and provided useful feedback to the research aims. A cross case-study analysis also helps to not only better 
understand the "whys" and "hows" of the various alliances, partnerships, and joint ventures but also the project management approach taken by these organizations in facing the new business environment. Different business realities under a common business environment help us understand the way trust, knowledge, and leadership played among stakeholders as well as the way culture played its role along the process and how the Corridor project facilitated this. This process provided fertile ground to examine the way the Corridor became a valid community of practice from where other communities of practice originated and developed by combining different aspects of knowledge, strategy, collaboration, cooperation, leadership, trust, and culture - all under a project management perspective.

A qualitative research methodology has been chosen in view of the specificity of the data gathered and the subjective nature of the information surveyed along with the author's direct participation and observation. This dual role has made possible the gathering of data from the inside of the Corridor Project as well as the identification of critical information arising out of a number of interviews and informal conversations.

The Corridor Project developed as a dynamic community of practice with project management office characteristics where a number of projects were born and evolved through their different life cycles to later turn into different business transformation outcomes. The research suggests that the Corridor could become a model to put into practice in other regions of the world where production and consumption asymmetries are found or where infrastructural asymmetries could call for a Corridor-like project to overcome trade imbalances or increase regional trade - all this leading to a series of business transformation opportunities to let corporations become more competitive by making a strategic use of knowledge sharing and transfer.

Keywords: Globalization; Knowledge Management; Community of Practice; Leadership; Collaboration; Trust. 
Table of contents

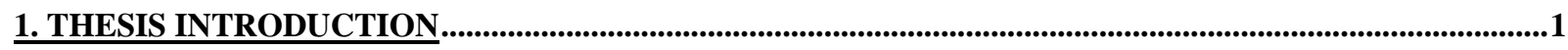

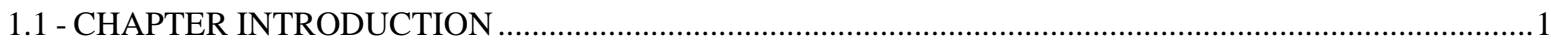

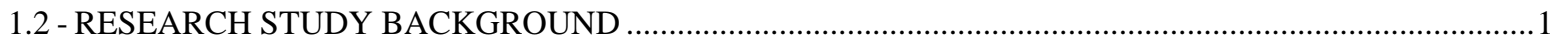

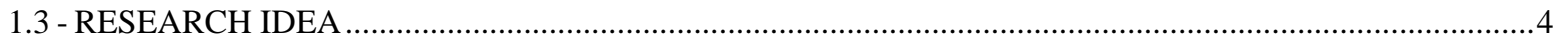

1.4- AIMS AND OBJECTIVES THAT FRAMED THE RESEARCH PROBLEM ............................................

1.5- INITIAL RESEARCH PROPOSITION AND RESEARCH QUESTIONS ...............................................

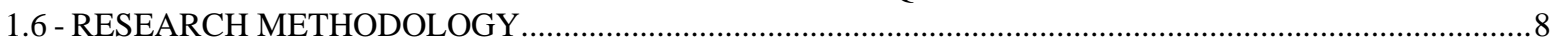

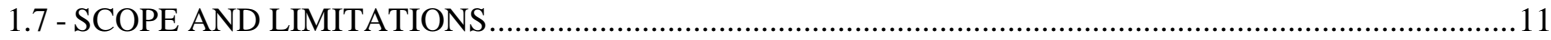

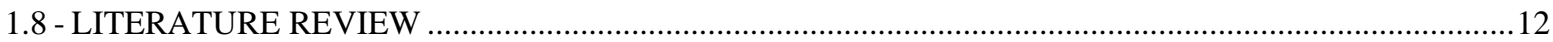

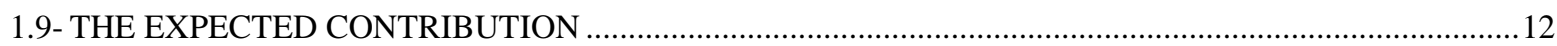

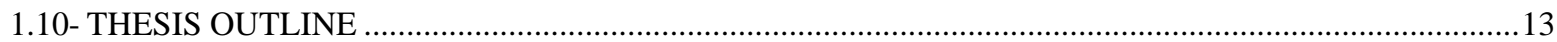

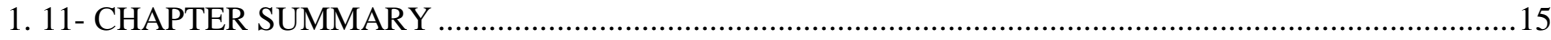

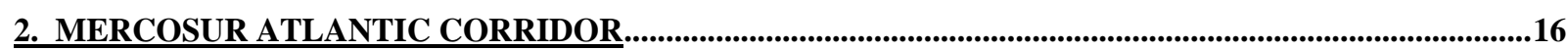

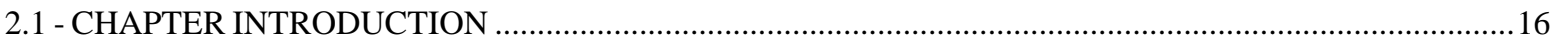

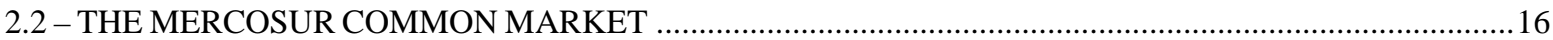

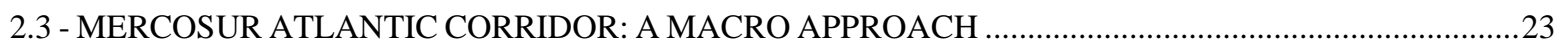

2.4 - MERCOSUR ATLANTIC CORRIDOR: A MICRO APPROACH ...........................................................27

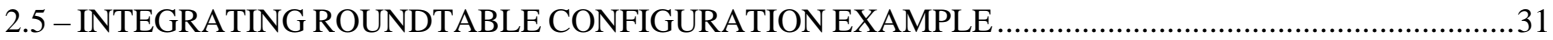

2.5.1 -Justification for the Corridor as an Entity Facilitating Strategic CoPs .................................................32

2.5.2- The Corridor Performing as a Strategic CoP .......................................................................................37

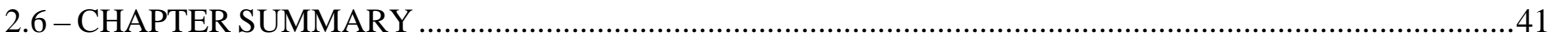

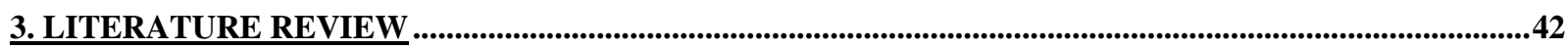

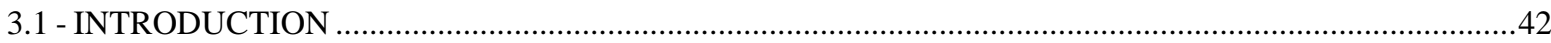

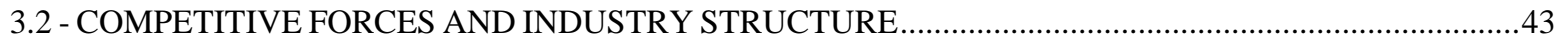

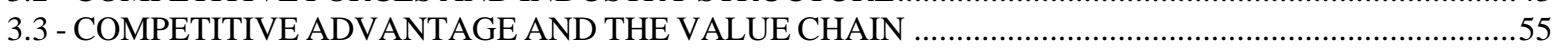

3.4 - THE THEORY OF THE FIRM AS IT APPLIES TO THE MERCOSUR REGION .....................................62

3.5 - LEADERSHIP AND STRATEGY THEORY TO MEET STAKEHOLDER NEEDS .................................70

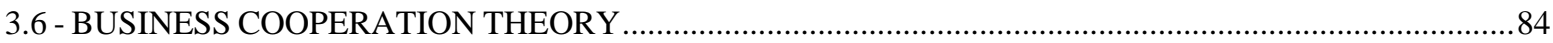

3.7 - KNOWLEDGE MANAGEMENT AND SITUATED LEARNING THEORY .....................................89

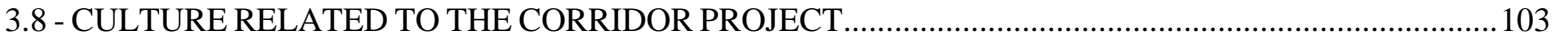

3.9 - PROJECT MANAGEMENT CONCEPTS APPLIED TO THE CORRIDOR PROJECT ............................109

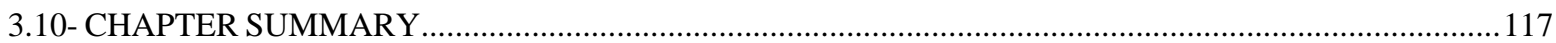

\section{THE CORRIDOR PROJECT: WORKING THROUGH COLLABORATION AND KNOWLEDGE}

TRANSFER .......................................................................................................................................................120

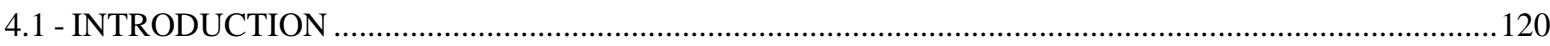

4.2 - THE CORRIDORAS A KNOWLEDGE TRANSFER DEVICE …....................................................121

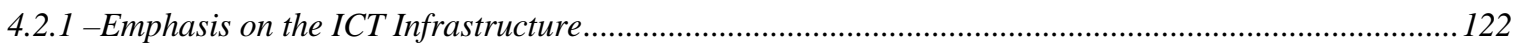

4.2.2- Emphasis on the Human Infrastructure ......................................................................................124

4.3 - COMPETITIVE ADVANTAGE THROUGH COLLABORATION AND COOPERATION .....................140

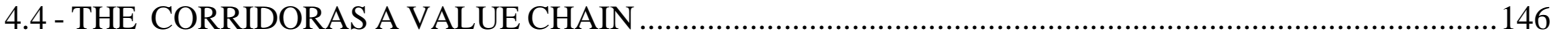

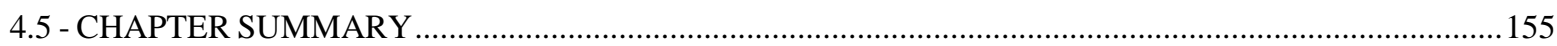

5. DISSERTATIONS, PROPOSITIONS AND RESEARCH METHODOLOGY …………................................162

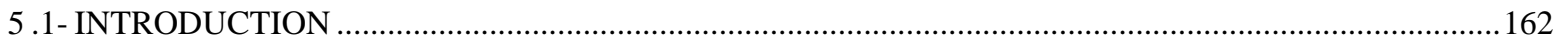

5.2 - RESEARCH PROPOSITION, STUDY AIMS, AND RESEARCH QUESTIONS .................................163

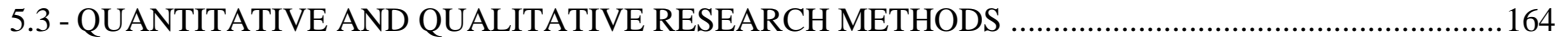

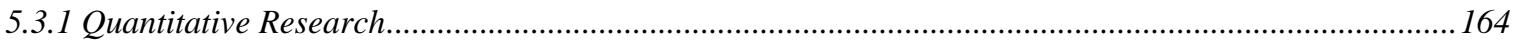

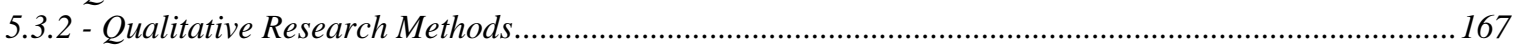

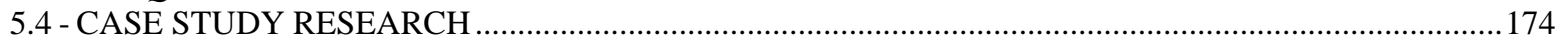




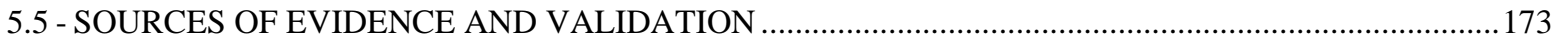

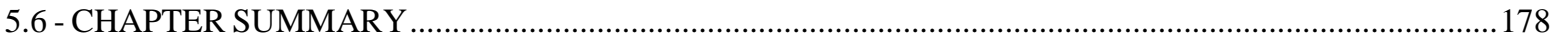

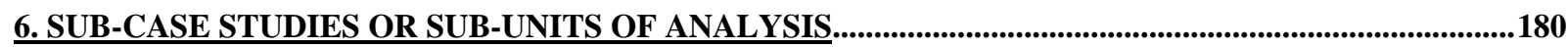

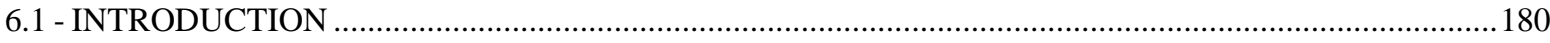

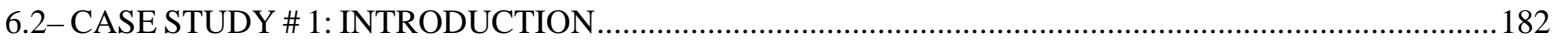

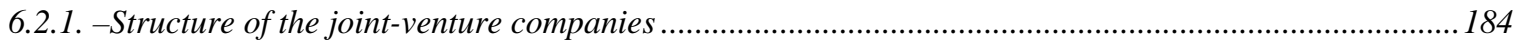

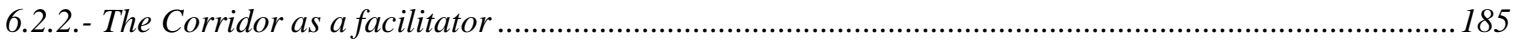

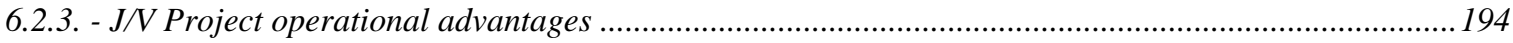

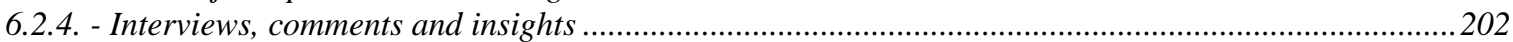

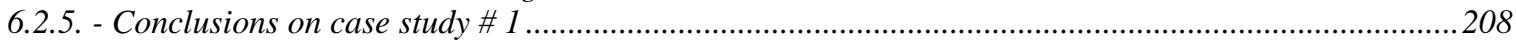

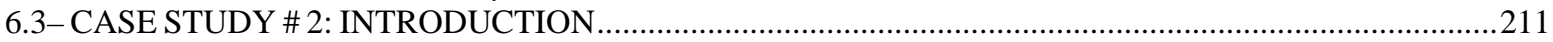

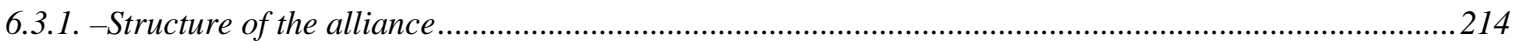

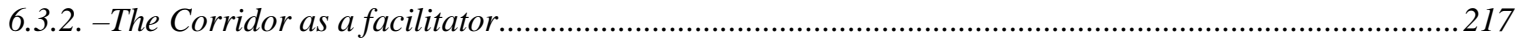

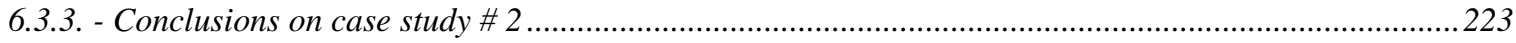

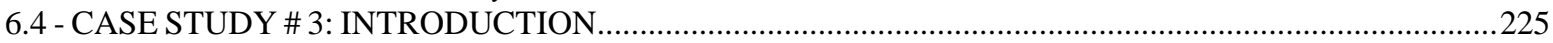

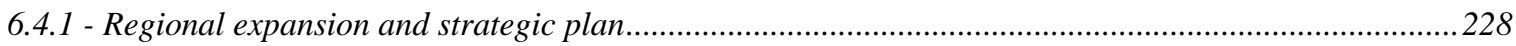

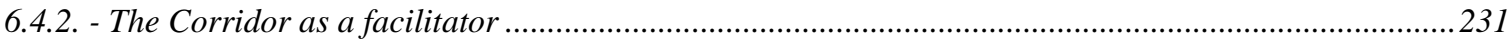

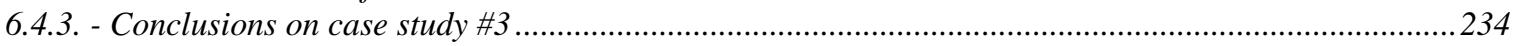

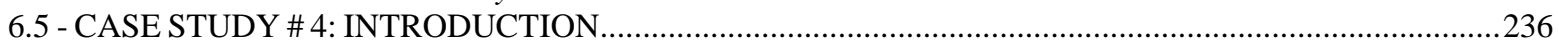

6.5.1. -Buenos Aires' Hinterland potential: A matter of knowledge and vision ............................................239

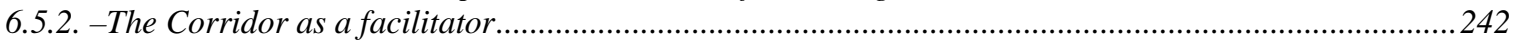

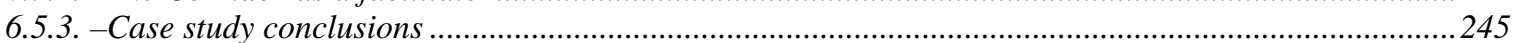

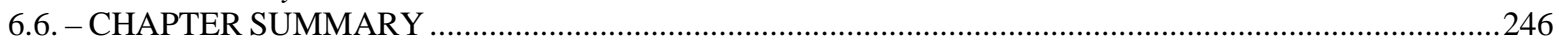

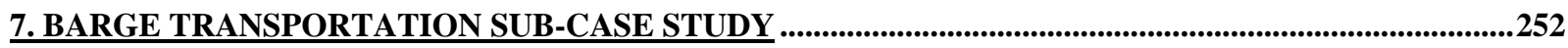

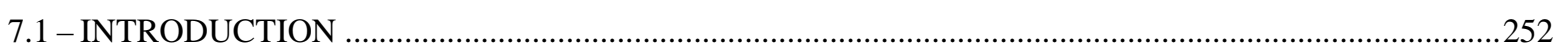

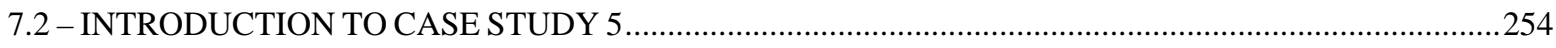

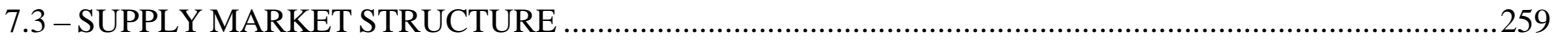

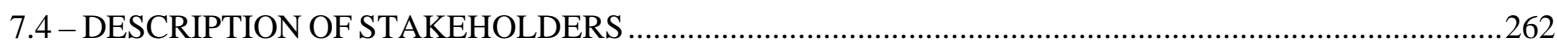

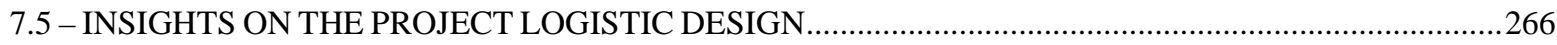

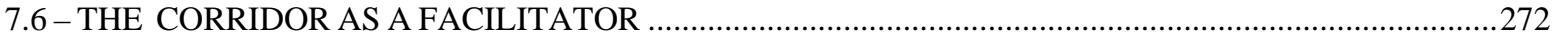

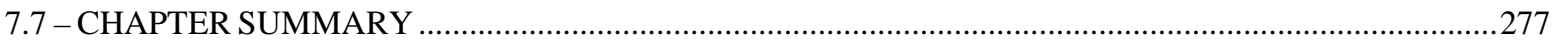

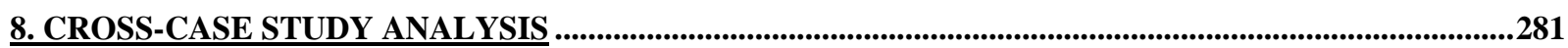

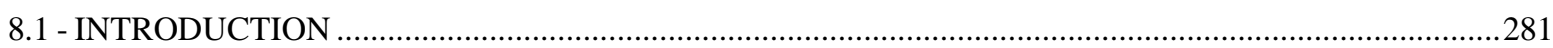

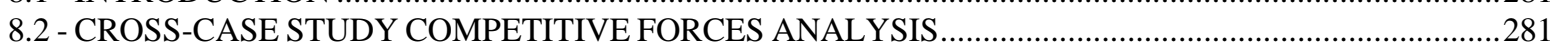

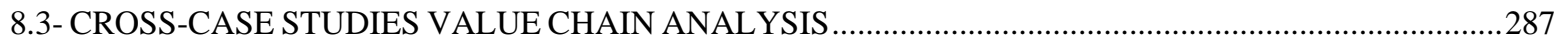

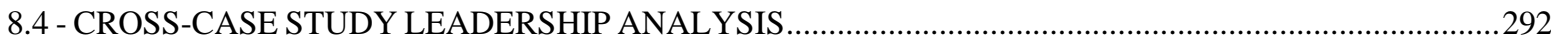

8.5- CROSS-CASE STUDY KNOWLEDGE MANAGEMENT ANALYSIS .................................................296

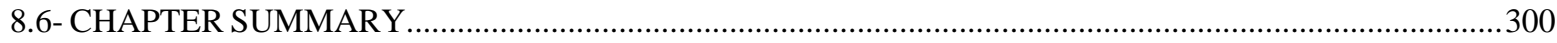

9. CORRIDOR'S PROJECT MANAGEMENT IMPROVEMENT NEEDS ....................................................303

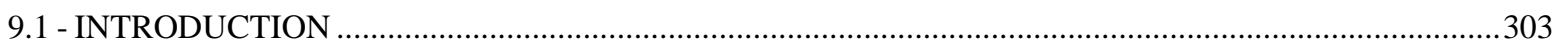

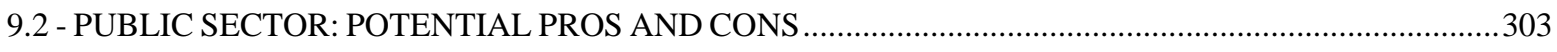

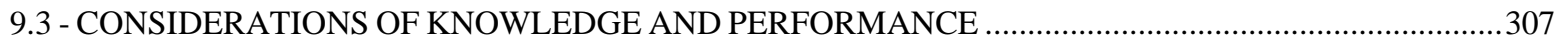

9.4 - CORRIDOR PM AND PUBLIC SECTOR ASPECTS CONCLUSIONS .................................................309

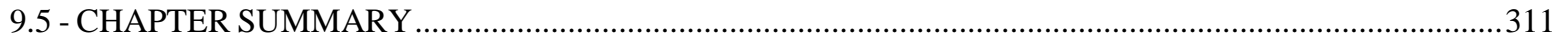

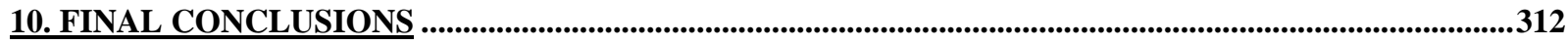

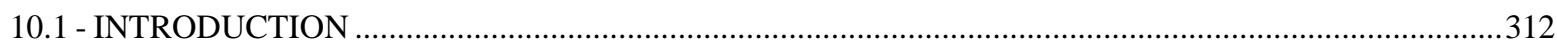

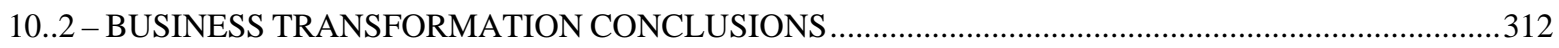

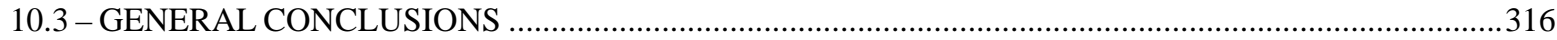

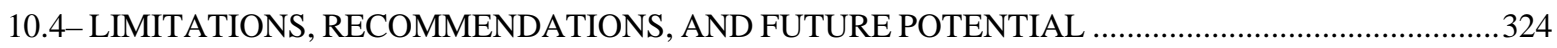

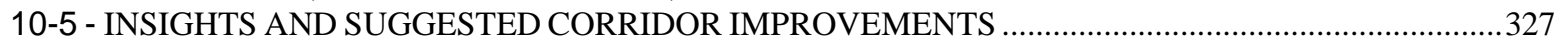

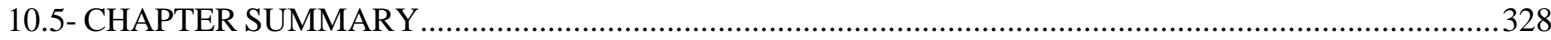




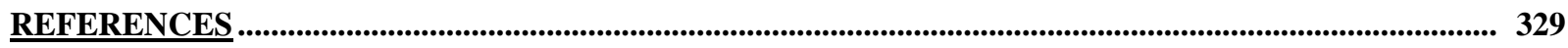

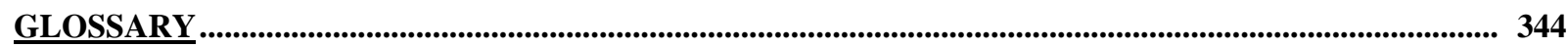

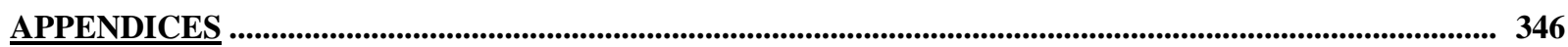

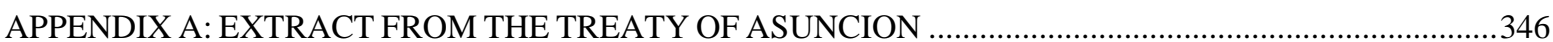

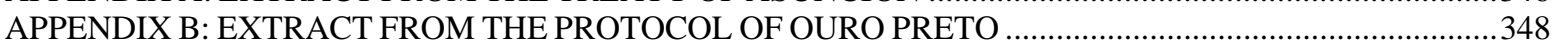

APPENDIX C: EXTRACT FROM THE CORRIDOR'S PROTOCOL OF INTENTIONS .................................350

Table of figures

Figure 1.1: DPM Learning Model and Outcome........................................................................... 3

Figure 1.2: Summary of Research Approach ............................................................................. 10

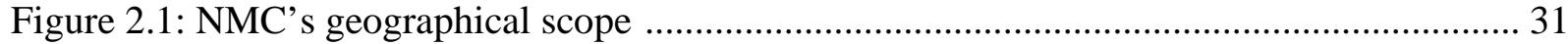

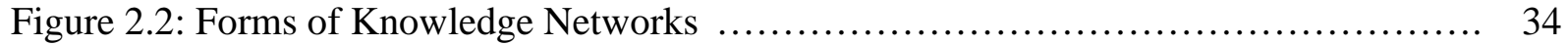

Figure 2.3: MERCOSUR Atlantic Corridor Consortium network ; 2004 ................................... 39

Figure 3.1: Understanding Corridor Development.................................................................... 44

Figure 3.2 Authority Sources and Leadership Style - Adapted from Tannenbaum and Schmidt

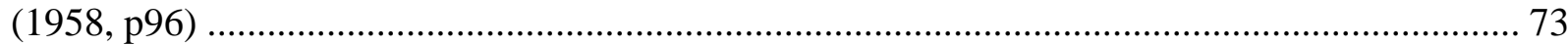

Figure 3.3 Leadership Styles and Follower Maturity: Adapted from (Hersey et al., 1996, p208) 74

Figure 3.4 Cultural Map of Latin America Cluster of Countries) ............................................... 109

Figure 4-1: The Corridor as a tool for collaborative sustainability ......................................... 121

Figure 4.2: Summary of the Role of the Corridor in response to the shock of global competition

and being shaped by the Region's culture, history and geography.......................................... 161

Figure 6.1: Joint Venture Formation Scenario to Gain Win-Win Outcomes from a

Rationalization and Effective Asset Re-Alignment Project ........................................................ 190

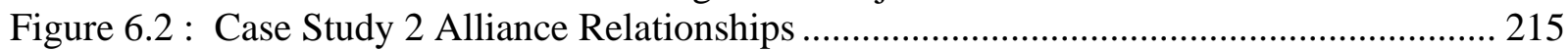

Figure 6.3 - Three stage ownership of case study railway organizations ................................... 227

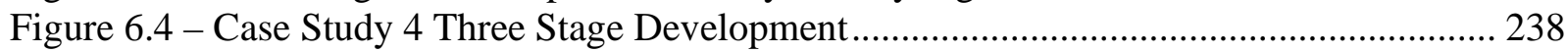

Figure 7-1: Barge carrier positioning barges in the River Plate.............................................. 260

Figure 7.2 : Typical barge convoy represented by multi-purpose barges based on informality and

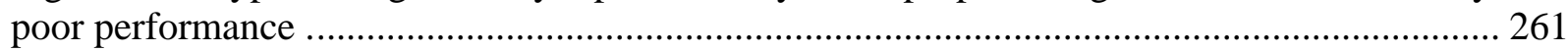

Figure 7-3: Joint Venture Showing Critical Primary Players and Instrumental Secondary Players

Figure 7-4 : Second Hand Barges Getting Ready to Start Loading Operations Alongside Berth in

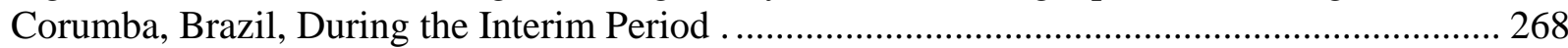

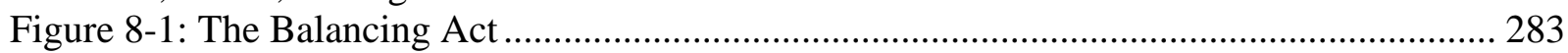

Figure 8.2 - Trust and Leadership Form and Style Influences ................................................ 293

Figure 9.1: The Government Participant Missing Link................................................................ 309

Figure 10.1: View of the Corridor's Role in JV Development .................................................... 323 
Table 4.1: Absorptive capacity example. ................................................................................. 133

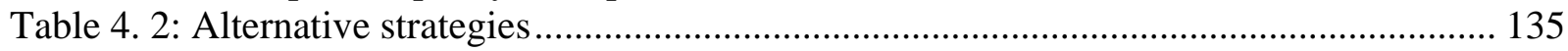

Table 6.1- Sources of Data for Case Study \#1 ........................................................................... 189

Table 6.2 - Shipping company's variable costs structure for 20 foot containers in South America for Export (above) and Import (below).................................................................................... 199

Table 6.3: Shipping company's variable costs structure for 40 foot containers in South America.

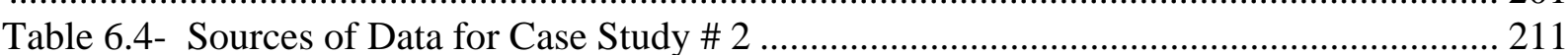

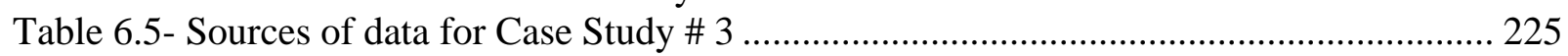

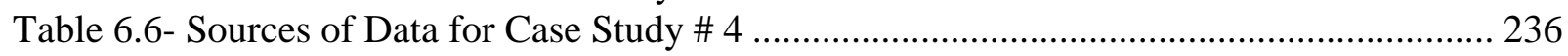

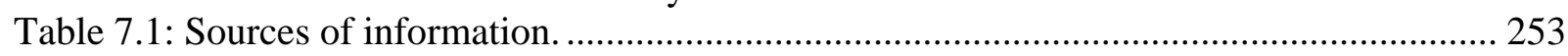

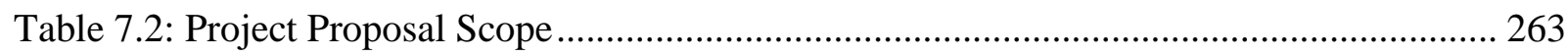

Table 8.1: Value Chain Analysis - Relevance of Porters’ Elements to Case Studies.................. 287

Table 10.1: Corridor: Transformation Facilitation Role Drivers and Inhibitors ......................... 314

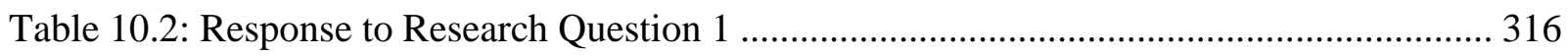

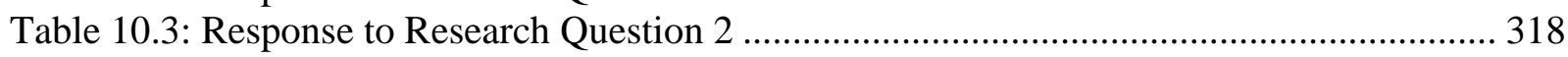

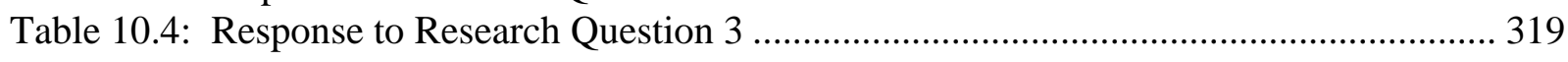

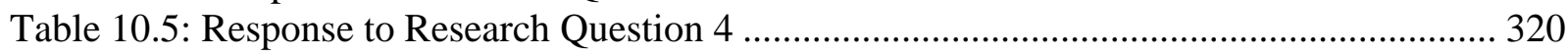

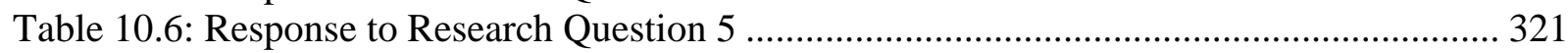

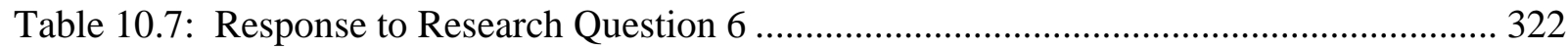




\section{Thesis Introduction}

\section{1 - CHAPTER INTRODUCTION}

This chapter commences with a brief description of the Doctor of Project Management (DPM). It then explains how the author used the core coursework phase of the DPM (exposure to the literature through the combination of reflective learning, action and field practice relating to the DPM's four areas of knowledge) to explore an innovative linkage between theory and practice that framed the choice of research topic-resulting in this thesis. An explanation on how the research idea evolved then follows, allowing the present research topic to develop, which was influenced by the coursework and by peers views and opinions together with field practice that facilitated interaction and experimentation that made this dissertation possible. An outline of the aims and objectives of this thesis is then provided, along with the research proposition and research questions. The next section concentrates on the overall research strategy and how it fits into a project setting. This is then followed by a description of the dissertation's scope and limitations, literature review within the defined fields of knowledge, and its expected contribution to better PM practice. The chapter concludes with a brief outline of the coming chapters and a final summary of the present chapter.

\section{2 - RESEARCH STUDY BACKGROUND}

The Doctor of Project Management (DPM) is a mixture of coursework (33\%) and research (67\%) where coursework is delivered on-line or in executive mode and research is undertaken in the candidate's workplace focused on a project management context (Graduate School of Business 2002, p2). The DPM program relies to a great extent on the research capabilities of the candidate and a great degree of commitment taken by the candidate to combine coursework, workload, and reflection on practice. All this will eventually lead the candidate to naturally link the research papers he/she has been working on throughout the years with aim of improving project work and project management practice. The program is intended to take project managers from their existing high levels of project management mastery to new dimensions of reflection on practice; through introducing academic rigour to the candidate's reflective learning process and linking his/her project management case study projects and individual knowledge and perspectives. 
The DPM program introduces the candidate to four new areas of knowledge that are at the cutting edge of project management practice and uses this new knowledge as a vehicle to develop project management wisdom (Graduate Business School 2002, p3). These are: Knowledge Management (KM) and innovation (Area 1); project management leadership topics such as stakeholder engagement, leading versus managing, cross cultural and team culture and strategy in a PM context (Area 2); Project procurement and Ethics (Area 3); and a further elective course, often tailored to the DPM candidate's research interest (Area 4).

The DPM combines reflective learning action on these four new areas of knowledge with work practice experiences focused on fieldwork and research on projects (in this case from all over South America), to bring about a genuine and useful linkage between theory and practice. This has resulted in the DPM experience being a difficult exercise to complete in view of the South American region's own cultural particulars and managerial characteristics, as discussed in the later chapters. On-site project management constraints, far from constituting obstacles for research, often provided fertile ground to experiment on and continuously exercise reflective learning while gathering rich feedback aiming at improving project management practice.

The author's experience of conducting the research study as described in this dissertation illustrates an interesting interaction between coursework, reflective learning action, and work practice that fits consistently into RMIT's professional doctorate aims and objectives for candidates to prepare a dissertation that is primarily based on the understanding of the overlap and interaction of action and its consequences leading to a consistent piece of research. The author's contribution is mainly based on considerable field experience and a permanent search of those linkages between theory and practice that result in worthwhile consideration of the betterment of project management practice in complex situations. Views and opinions of project management peers at RMIT along with the coursework outcomes have exerted a clear influence upon the author with respect to taking into account not only different perspectives or professional opinions, but also to include the cultural factor and the extent to which knowledge management is valued and put into practice. 


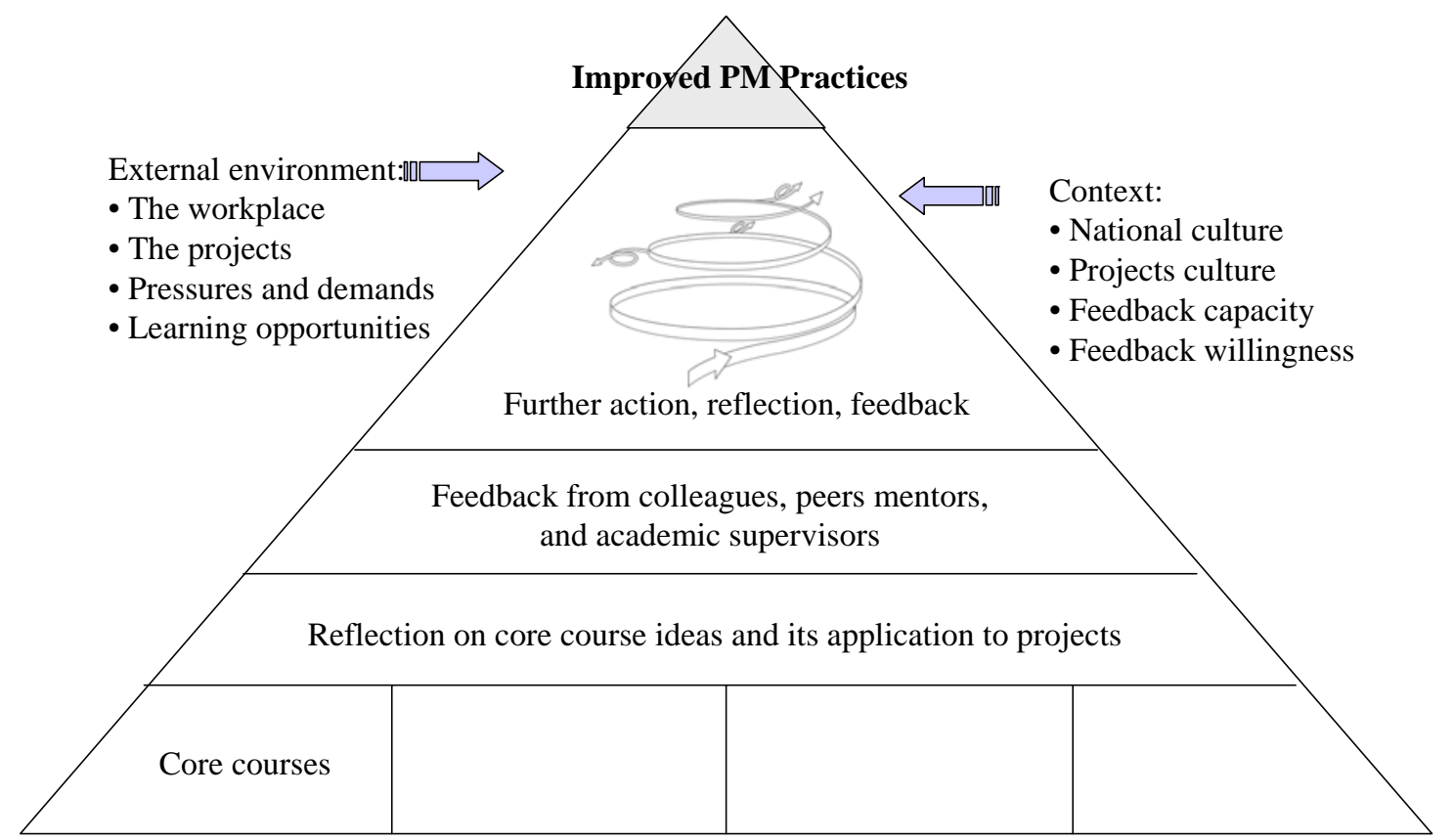

Figure 1.1: DPM Learning Model and Outcome

Figure 1.1 illustrates the learning model undertaken using the DPM in this thesis and its outcome. The author brings experience and insights from various large industrial projects in South America centered on hydrocarbons, mining, and international transportation industry segments where both his direct observation and direct participation resulted in the pyramidal model illustrated in Figure 1.1. Coursework provided a foundation upon which subsequent interactions of reflection and feedback provided a fruitful outcome between coursework, peers views and impressions, reflective learning using an action research approach. Action research is described by McNiff and Whitehead (2000, p203) as starting with the question "How do I improve my work? It requires the researcher to be central to the research process, to embody selflearning and self-transformation through that learning, not aiming for closure but ongoing development, participating in the research rather than being an independent observer and focusing on the process being educational. There are a number of forms of action research that will be discussed later in Chapter 5 . This work uses a case study in which the author was a participant. The most important aspect of the dissertation however, is possibly represented by what Kurt Lewin, in his classic formulation of field theory (Lewin,1951) defined in the sense that behavior is influenced by its environment and the context within which it occurs (Reason \& Bradbury 2001, p38) - a topic of utmost importance in project management. Coursework at 
RMIT provided the foundation of the pyramid upon which, the workplace largely served as the site for undertaking research (Malfroy 2004, pp68-70). The results are improved project management practice employed by the candidate and disseminated more widely to colleagues and the PM profession.

As CEO of a consulting organization that had, through reputation and role in the Corridor project, enabled me to influence colleagues and peers within the Corridor entity.

\section{3 - RESEARCH IDEA}

The research idea arrived at for this thesis was chosen from one of several projects that I have been involved with that were of interest in terms of my DPM journey of reflection.

The basic idea of the present dissertation originated through combining my experience in one of the major projects he participated in with the ideas generated from the Project Procurement and Ethics course. During this coursework I had the opportunity to investigate two key management fields: sustainable development (that is, considering not only the financial, but also the social and environmental impact to ensure long term viability) with all its ethics components, and alliances and partnerships as a way to focus on valueenhancement and resource use effectiveness through genuine corporate collaboration. By then, I was immersed in South America's largest gas fields project in Peru (the Camisea project) and in two gas plant expansions project in south eastern Bolivia (San Alberto I \& II). The coursework exerted a definite influence on the way I started to look at the mode the coursework subjects were dealt with at the respective job-sites. Thus, gradually I took advantage of my direct participation in both projects to observe relevant events in detail, gather related data, and talk to people on-site with appropriate knowledge and insights to help make sense of what was taking place there. These insights included the way that leadership was exercised; the way indigenous communities were hired and treated; the way project activities impacted on the environment and the way the contingency planning worked or did not work; the feelings and attitudes of people; cultural insights and personal perceptions hidden behind people's way of looking and behaving before unfriendly contexts etc. By then, I mentally constructed ideas for a potential contribution for better 
project management practice in terms of ethics and sustainable development, while carefully looking into the extent to which project partnerships and alliances might result in better site management practice. I scrutinized real events and situations, observed at the various job-sites, and contrasted these with coursework learning materials along with RMIT peers' views and opinions, always with the purpose of bringing to light a different perspective to confront these rather unique situations.

The knowledge management (KM) coursework was of paramount importance to me as it gave me the opportunity to relate knowledge transfer and sharing concepts within the MERCOSUR Atlantic Corridor project (henceforth referred to as 'the Corridor')- a project where corporate interactions within various dimensions led to alliances, partnerships, economies, and change. Further, this project was significantly complex and unique enough to incorporate ideas and from coursework topics the author had completed within the field of Project Procurement and Ethics. Finally, the "leadership" coursework constituted one of the pillars of the dissertation in view of its intrinsic importance as regards to transactional and transformational dimensions together with the leader-follower interrelationship in a project that involved large multinational players in the mining and steel industry segments - always in South America. Finally, it was the combination of the above mentioned coursework topics which fitted consistently into a regional project that embodied a large number of players (participant individual people and firms) and therefore diverse scenarios. In this way, I naturally found a dissertation topic that allows the inclusion and interaction of coursework subjects and a real connection with a project which is characterized by diversity, complexity, and uniqueness - all within a region where normally project management practice experience recurrent barriers to good practice.

\section{4- CORRIDOR AIMS AND OBJECTIVES THAT FRAMED THE RESEARCH PROBLEM}

The aim of this study is to establish how a huge geographical region along with a growing number of players can re-engineer its business methods to encourage intra-regional cooperation by focusing on an efficient knowledge management while exercising a different leadership style to that normally adopted in the region. Improving the culture of cooperation of the Corridor partners turns out of paramount importance encouraging further effective medium and long-term 
collaboration to improve the MERCOSUR economies and competitiveness as well as valuegeneration of its participating organizations. This aim entails gradual acceptance and putting into practice a deep regional business transformation by increasing the number and quality of participants who - to a greater or lesser extent - saw themselves compelled to overcome a number of barriers that were imposed by contextual circumstances (to be more fully discussed in Chapter 2).

Therefore this thesis focuses on, and seeks to understand, the following Corridor project objectives that led to conceptualizing it as a strategic community of practice $^{1}(\mathrm{CoP})$ and therefore helped shape the research questions:

1. Improving cooperation between Corridor participants to enable the creation of a knowledge marketplace aiming at gaining competitive advantage for individual organizations and Corridor participants;

2. Defending participants' market share and further improving it by developing a timely reaction capacity to global business trends impacting on the region;

3. Fostering collaboration to overcome regional infrastructure deficiencies and further achieve both regional and global-oriented economies of scale;

4. Facilitating the identification of business opportunities at the regional level and promoting the access of both service and infrastructure-related foreign direct investment; and

5. Strengthening intra-regional trade by linking remote locations with consumption poles within the region while increasing overall regional economies of scale.

The first objective has to do with knowledge sharing and transfer as a vehicle towards reducing negative impacts to business cooperation and enhancing trust and cooperation between the Corridor participants through the so called "integration round-tables". These periodical gatherings served as fertile ground to encourage corporations get in touch with one another and share knowledge in search of achieving a common competitive advantage under some sort of win-win scenario. The second objective is directly related to the effects globalization trends had on the region where the Corridor, along with its communities of practice (CoP) — see Section 2.5 for support of this assertion, induced participants to face business transformation successfully and

\footnotetext{
${ }^{1}$ See Section 2.5 for a full explanation of this term
} 
coordinate their regional responses by learning how to cope with change, while making good use of innovation strategies. The third objective raises once more, the importance of regional cooperation and value enhancement through CoP, by not only focusing on achieving regional economies of scale but also enlarging these and expanding their global reach. The fourth objective aims at promoting better project management practice upon identifying niche markets where direct investment helps improving and expanding the existing level of infrastructure as a way to become the whole system more viable, sustainable and competitive at the regional level to be able to face the global trends. The last objective brings to the discussion table what probably was the very first target of the Corridor, which was always aimed at bringing remotely located organizations and resources closer to consumption centers by making use of innovative procurement, leadership, change, and cross-cultural management techniques and practices.

\section{5- INITIAL RESEARCH PROPOSITION AND RESEARCH QUESTIONS}

Based on the Corridor's aims and objectives that framed the research idea, the research proposition is that the MERCOSUR Atlantic Corridor Project becomes an instrumental entity where regional stakeholders find a useful system and the adequate tools to formulate and implement a business reengineering strategy towards achieving larger economies of scale. As such, the system entails stakeholders to engage in a cultural transformation journey, aiming at overcoming trans-national operational deficiencies and gaining regional competitive advantage. Consequently, a number of research questions naturally present themselves in connection with the proposition and the embodied aims and objectives of the dissertation:

- $\quad$ Research Question 1:

What are the variables granting the Corridor project the ability to generate trust first and facilitate knowledge transfer among stakeholders?

- $\quad$ Research Question 2:

How do Corridor stakeholders share vital internal information and join forces to face extraregional threats?

- $\quad$ Research Question 3:

Why do Corridor stakeholders show their current attitudes and motivations toward committing themselves with partnerships, alliances, and joint-ventures?

- $\quad$ Research Question 4: 
How does culture play a role in easing or impeding the development of the Corridor vision throughout the region?

- $\quad$ Research Question 5:

What factors hold a more relative influence on stakeholders to allow vertical or horizontal integration to occur?

- $\quad$ Research Question 6:

What is the actual awareness degree among stakeholders with respect to sustainable development and its future relevance over the region?

\section{6 - RESEARCH METHODOLOGY}

The selected research approach is that of a single case study in view of its uniqueness and great number of variables that are embodied in the project. As such, this case study is both descriptive and explanatory. It contains an exploratory phase as there is no similar project in the world with the exception of the North Atlantic Maritime Corridor, which is more recent, more modest in its objectives, and more limited in its scope of action. Case studies may be exploratory, descriptive, or explanatory (Yin 1994, p4). Even though each case study strategy has its distinctive characteristics, there are large areas of overlap among them (Sieber 1973). The type of research questions posed in the dissertation makes it necessary to deepen the explanatory and exploratory dimensions, though without excluding the descriptive stage in view of the project rareness or uniqueness. A case study is an empirical inquiry that investigates a contemporary phenomenon within its real-life context, especially when the boundaries between phenomenon and context are not clearly evident (Yin 1994, p13).

The MERCOSUR Atlantic Corridor project is developed as a single case study though it embodies various units of analysis, which, owing to their magnitude and extent, are presented as sub-case studies or sub-units of analysis. These subunits of analysis are not intended to serve as literal or theoretical replication, but to help demonstrate the propositions by addressing the research questions within a pattern-matching dimension, and by incorporating different angles and conclusions arising out of the subunits. This situation leads the dissertation to look more like a multiple-case design despite containing the three basic elements of a single-case design: critical test ; unique case ; revelatory case (Yin 1994, p45). The evidence of multiple cases is often 
considered more compelling, and the overall study is therefore regarded as being more robust (Herriot \& Firestone,1983). It is also a case that because of its uniqueness or rareness perhaps, this can be considered to be also revelatory as it allows knowledge, culture, procurement, ethics, and leadership to interact and innovate within a transnational environment. Yin (1994 p40) argues that the revelatory condition exists when an investigator has the opportunity to observe and analyze a phenomenon previously inaccessible to scientific information.

Multiple data collection methods are utilized including literature review in three languages, individual interviewing of key players at regional level in an open-ended fashion, desk-based investigation of monthly minutes, and archival records analysis - further to what the author regards as of paramount importance: direct observation and direct participation. These are the main sources of evidence, though a more detailed explanation will be made available in Chapter 5 of this dissertation - as well as of the research strategy which is been chosen to be of a qualitative nature. A qualitative research strategy requires your direct, personal engagement with the field, its actors and the archives, and one that challenges you to grapple with "real world" complexity, interaction and change (Burton \& Steane 2004, p161). A grounded theory approach was selected as the most convenient way to analyze the data. This is because of its strength of inductive generation and discovery of theory from collected and analyzed field data that leads to interpretation of a phenomenon in its contextual surroundings (Burton \& Steane 2004, p170).

The research findings of this dissertation are designed to avoid any kind of harm for the participants. While some of participants agreed to be named others did not, so respect for the integrity of those participants was maintained with both confidentiality and anonymity. The research is conducted by the full observance of the principles of informed consent, honesty, conflict of interest and privacy (Burton \& Steane 2004, pp63-70). Ethics approval was sought and obtained through the normal RMIT procedures.

The research approach can be summarized in Figure 1.2 as follows. 


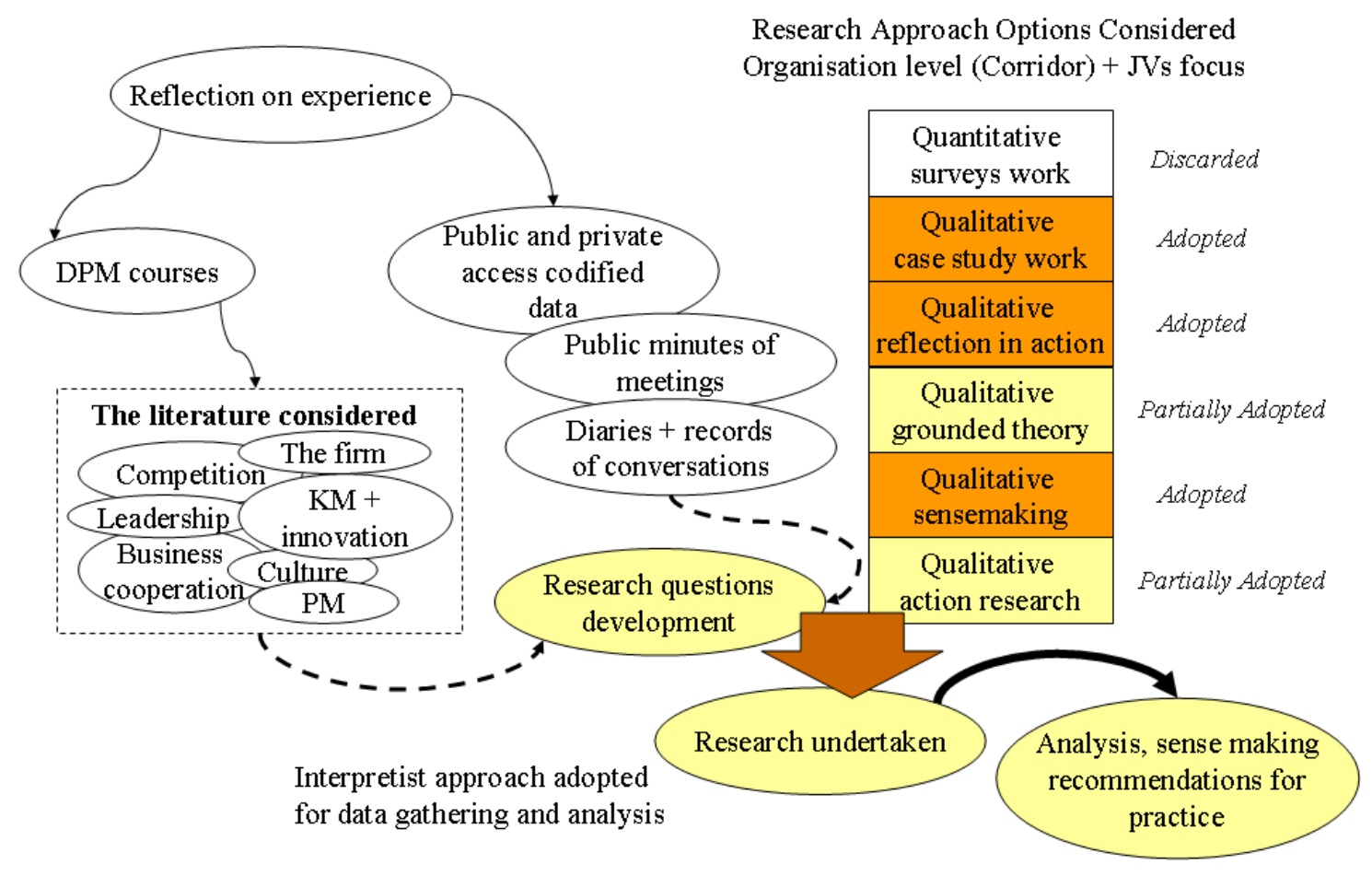

Figure 1-2 Summary of the Research Approach

The principal focus for the research was how the Atlantic Corridor project functions as an entity and a means to develop business transformations of it members. This was best conducted by viewing the Corridor as a single case study entity investigated through a series of sub-case studies of joint ventures (JVs) that were facilitated through the Corridor.

The explication of the research questions came after much reflection during exposure to theory while undertaking the DPM core courses. They were distilled from further review of literature already accessed during the coursework stage and this combined with preliminary data gathered over a number of years allowed me to crystallize the research questions so that the research proposition that is linked to the nature of the Corridor as an entity and how it was shaped by global and local events to provide a vehicle for business transformation that provided participant firms with a competitive advantage to sustain their business interests. The proposition led to a series of aims identified in Section 1.4. These in turn presented an opportunity, given access to data illustrated in Figure 1-2 above, to scope the research into a manageable scale that could be undertaken by a single researcher within the expectations of the DPM thesis requirements. 
Various research approach options have been described and illustrated in Figure 1-2 above. The case study approach was selected with some reflection in action as a participant that allowed me access to data as well as main players involved to verify and validate opinion expressed, data gathered, conclusions drawn and implications implied from analysis of the case studies. Interpretation of data to draw conclusions from the data was based on use of some grounded theory approaches to highlight themes and meanings and I used a sense-making approach to develop conclusions. These approaches are explained in more detail in Chapter 5.

\section{7 - SCOPE AND LIMITATIONS}

I believe in capitalizing on the distinctive opportunity to perceive reality from the viewpoint of someone inside the case study rather than taking the role of a typically passive external observer. However, I am also well aware of problems the condition of participant-observer may bring with respect to potential biases, and therefore I intend creating transparency of the findings and conclusions by adding multiple sources of evidence. I also recognize and acknowledge that being familiar with the subject of the dissertation (and in the struggle to introduce only relevant valuable material and discard what I deem too elementary or basic) I might have left out some crucial background or contextual information at any given stage.

Evans (1995) describes this tendency as the so-called "95 per cent syndrome" where a critical 5 per cent may be left out because it seems obvious. Even though my direct participation and observation of many of the crucial events that have shaped this project (and despite the potential emotional ties I might have held throughout its development) this did not hinder me from utilizing multiple sources of evidence as a practical way to offset such a potential threat and bring balance to the research study.

The research methodology is of a qualitative nature, where a limited number of open-ended interviews were conducted. However, my direct participation and observation introduced a number of inferences which were duly recorded and later validated by multiple-source evidence. Even though the Corridor members developed into a growing number of stakeholders, meaning that a quantitative approach would have been possible to combine with a qualitative research, this would have turned out impractical and too time-consuming for collecting quantitative data. 


\section{8 - LITERATURE REVIEW}

I chose English language literature sources as the most appropriate, with some references found from Portuguese and Spanish language literature. The research study embodies a number of leading subjects that help shape the research idea, its aims and objectives. These include:

$\begin{array}{ll}\text { - } & \text { Theory of the firm and competitive advantage; } \\ \text { - } & \text { Leadership: transformational and transactional; leader-follower. } \\ \text { - } & \text { Change and Innovation management, procurement. } \\ \text { - } & \text { Knowledge management: knowledge sharing and transfer. } \\ \text { - } & \text { Cross-cultural management: transnational impact. }\end{array}$

The literature review helps substantiate the case study data along with the subunits of analysis with respect to the above main segments of study. This adds value to understanding the Corridor's search for enhanced competitive advantage and enlarged regional economies of scale.

\section{9- THE EXPECTED CONTRIBUTION}

This thesis contributes useful PM research that Winter, Smith, Morris and Cicmil (2006, p642) highlights the need for. More specifically, they stress the need for theory for PM practice directions 2, (projects as social processes), 3 (value creation as the primary focus) and 4 (broader conceptualizations of projects) as well as research direction 5 (towards practitioners as reflective practitioners). This thesis addresses those research directions.

Their aim was to encourage PM researchers to focus on projects as part of a lived experience with human factors significantly affecting project outcomes (direction 2) — this thesis is focused very much on participant experiences and perceptions. They also highlight direction 3 as a way for PM researchers to think beyond project success in terms of time, cost and quality but as a means of creating benefits and value-business transformation and value chain improvement is also highlighted in this thesis. Direction 4 relates to broader conceptualization of projects and this thesis looks at the Corridor as a program of projects. Finally their direction 5 calls for reflective practitioners and I this helped me to become one through this research study. 
Therefore, following the approach suggested by Winter et al. (2006) explained above, this research into the Corridor provides a number of expected contributions to facilitate improved PM practice that can be identified as follows:

- $\quad$ Providing an opportunity to explore and better understand how leadership, culture, and knowledge sharing and transfer interacted and produced both tangible and intangible regional benefits.

- $\quad$ Providing a useful mechanism that challenges the typical leader-follower relationship and brings about a new approach for the betterment of the primary stakeholders strategic position within the region.

- $\quad$ Establishing a new organizational model at regional level that facilitates understanding and, better confronting, the often unstoppable global business trends for the benefit of both primary and secondary stakeholders.

- $\quad$ Showing the way regional physical and psychological constraints or limitations can be overcome and implemented in other geographies under similar parameters to produce both tangible benefits and intangible improvements.

\subsection{0- THESIS OUTLINE}

Chapter one sets the context of the research by focusing on the role played by the interaction of coursework and on-field practice, the development of the research idea, and the formulation of the thesis' aims and objectives. It further refers to and briefly explains the initial research proposition and research questions to later provide the framework of the chosen research methodology along with the author's view of what the scope of the work is and the existing potential limitations.

Chapter two discusses the MERCOSUR Atlantic Corridor from an historical perspective by briefly describing its evolution, and the context into which it is immersed to let the reader understand its magnitude and potential. It explains how it works in practice and identifies various linkages with leadership and social network theory that result valuable to adequately address the research questions. Chapter 2 forms a contextual basis from which the research data can be made better sense of. 
Chapter three reviews the existing literature concerning knowledge management, leadership, cross-cultural and change management, and procurement, all with respect to a large and unique regional or trans-national project of this type. Gaps in the literature are identified and subsequently utilized to address every research question.

Chapter four describes the Corridor in terms of its role as a program/portfolio tool that enables projects to be realized through facilitating collaboration, cooperation, and knowledge sharing. It also establishes the importance of the Corridor as a value chain promoter.

Chapter five addresses and analyses the various research methodologies available and proceeds to justify the chosen methodology in view of its suitability with respect to the research questions, always within the framework of the thesis aims and objectives. It provides a comprehensive approach to data collection; it explains how the process leads to classify and sort out the findings; and it further provides an explanation on how bias is overcome and how accuracy issues are dealt with.

Chapter six refers to a number of sub-cases that form part of a selected cluster, where specific short-term barriers or constraints were cooperatively overcome, leading stakeholders to gain competitive advantage and enlarge economies of scale. The findings arising out of the data collection process are contrasted with theory and pertinent conclusion are assessed and presented. The chapter also presents alternative world scenarios where the Corridor concept might be put into practice elsewhere under similar parameters.

Chapter seven focuses on a complex long term case study where the existing constraints entail stakeholders carefully evaluate not only the way to gain competitive advantage and enlarge economies of scale, but also how to cope with their long term strategic positioning problem and intangible resource management threat. As with the previous chapter, findings and theory are contrasted and discussed while an outline of lessons learned are provided. 
Chapter eight develops a cross-case study analysis by considering Porter's (1985) three main strategies and value chain model as a way to identify the actual contribution of the Corridor to the different roles played by both the primary and support activities.

Chapter nine addresses the Corridor's needs of improvement from a project management perspective and with a particular emphasis on the role played by the public sector, along with its potential impact on the regional development.

Chapter ten summarizes the research findings, highlights a number of insights, and provides a number of suggestions in the form of final conclusions. A series of appendices display samples of supporting evidence associated with this research study.

\section{11- CHAPTER SUMMARY}

This chapter has provided all the necessary elements to inform the reader of the content and structure of the following chapters. It presented the research initial idea, followed by the thesis aims and objectives, and followed this with the detail about the research proposition and research questions. The proposed methodology is identified with guidance about how bias will be overcome and the scope of the work was also described. Finally, a thesis outline is provided along with a brief description of what each chapter contains as a way to facilitate the reader to comprehend the sequence of the work. 


\section{MERCOSUR ATLANTIC CORRIDOR}

\section{1 - CHAPTER INTRODUCTION}

This chapter commences with an introduction to the main facts that gave birth to the MERCOSUR Atlantic Corridor by describing and discussing first the nature of the MERCOSUR common market itself, and to later briefly describe two different perspectives on the Atlantic Corridor Project: a macro-approach and a micro-approach. These different views help the reader more fully understand the potentialities of the project which will be more fully explored and analyzed in the upcoming chapters. The chapter concludes with a summary of this chapter's main points.

\section{2 - THE MERCOSUR COMMON MARKET}

South America's salient feature, besides its vast continental mass, is the existence of a very large central region scarcely inhabited and loosely associated to the continent's own economy and that of the world at large. The center of the continent encompasses several important ecosystems sequentially succeeding each other from the equator up north to the deep polar southcomposing a remarkable inland mass encompassing the Amazon Basin, the Cerrados, the Pantanal, Chaco, Pampas, and Patagonia. It is very well endowed with good agricultural soils, mineral reserves, forests, oil, gas, and large rivers meaning water, waterways, and hydropower potential in the range of hundreds of thousands megawatts.

South America's major economic and population centers and areas are concentrated in its coastal areas, facing outwardly from the continent. As a consequence, the intra-continental connections between its oceanic borders are both limited and inadequate in most cases. However, the trade between its Atlantic and Pacific economies are modest when compared to its actual potential. This situation developed from historical, cultural, and geographical factors-for example the natural barriers represented by the Andes cordillera and the Amazon rainforest, or the various existing languages and dialects across bordering nations and even within the same country. 
The advent of the MERCOSUR common market, the Andean Pact, and other important integrative initiatives have been gradually changing the traditional mindset and has led toward a program of projects that contribute to a shared destiny for the many countries of South America. This brings to light an urgent need for better communications and transportation systems. The MERCOSUR common market had a promising genesis in 1986 when four of its original members decided to join efforts to enhance overall intra-regional economies of scale and increase their joint negotiating power as a bloc. Argentina, Brazil, Paraguay, and Uruguay were the founding partners whereas Bolivia and Chile joined later under a differentiated status. Currently Venezuela, Mexico, and Peru are considering in joining the bloc, subject to political consideration - and later South Africa as the first outer regional member.

MERCOSUR, the “Common Market of the Southern Cone,” was created in March 1990 by the Asuncion Treaty and was meant to create a common market among its four signatories (Argentina, Brazil, Paraguay, and Uruguay) by December 31st 1994 (Appendix A). This common market would include a gradual elimination of all custom duties among its signatories, the creation of a common external tariff along with the adoption of a common trade policy, and the harmonization of economic policies.

The Treaty of Asuncion and its supplementary Protocol of Ouro Preto brought about the creation of a number of institutions to assist in the process of attaining these goals (Appendix B). Since its beginnings, MERCOSUR has achieved more than any of the previously existing economic bloc organizations (e.g. Latin American Free Trade Association ; Andean Pact ; Caribbean Community) as it has formalized and expanded cooperation and trading relationships to become a vibrant and viable economic integration organization. For a substantial period of time, its members enjoyed unprecedented expanded trade and great prosperity (Cohen \& McClusky 1996). Indeed, according to the International Trade Reporter intra-MERCOSUR trade increased 314\%, to \$17.1 billion, during 1990-1996 and reached a peak of \$20.5 billion in 1997-1998.

Institutionally, the MERCOSUR has agreed on a common external tariff covering 85\% of imports currently being traded by its members and reached agreement on a substantial number of trade matters. Thus, it can be argued that it has generated a significant amount of excitement 
among the elites of its member states, who step-by-step leant toward the idea of economic integration as both feasible and desirable. The bloc managed to recruit two new regional members (Chile and Bolivia) and later entered into a substantial cooperative relationship with both the European Union and a number of different organizations and individual extra-regional countries. Even Argentine ex-President Carlos Menem - as explained frequently in the

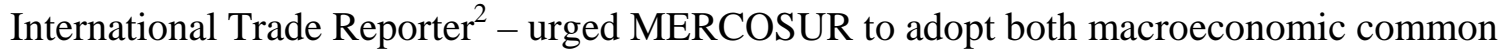
patterns and a single regional currency.

This optimistic environment has changed somewhat since 1999 as severe economic difficulties became apparent first in Brazil, then in Argentina and the other member states. This had a tremendous impact on the MERCOSUR and its development. A clear assessment made at the time by the Brazilian ex-President Fernando Henrique Cardoso who stated that while there was a lot to be sorry about, there was one issue that trumped all others-inflation. Prices in Brazil in 1993 rose an astonishing 2,500\% . This kind of hyperinflation is difficult to imagine for someone who has never lived through it. It dominates business and daily life. On pay day, people lined up outside supermarkets, desperate to spend their money before it lost its value. Prices on basic goods such as rice (the Brazilian staple) could double in just a day. All contracts, bank accounts, tax bills and salaries had to be adjusted for inflation. This was an imprecise process encouraging tremendous corruption and, as described by Cardoso (2006 ; 173-7), was economic hell.

Further, the regional economy seemed also to be trapped in a stop-go policy cycle that imposed a detrimental impact on regional trade. Also at that time the MERCOSUR faced a hemispheric competitor - USA - that was actively sponsoring the Free Trade Association of the Americas, better known as FTAA or ALCA for all Latin America. However as de Jonquiers (2001) argued, “... regardless of the region's economic difficulties and potential competition from another organization, MERCOSUR members apparently remain committed to the concept of regional economic integration in general and to the maintenance and development of MERCOSUR in particular. Indeed, regional trade organizations like MERCOSUR have emerged as a driving force in the global economy."

\footnotetext{
${ }^{2}$ Comments held on various occasions during Carlos Menem,s first term from December 1989 to December 1994.
} 
An introduction to the MERCOSUR Common Market would not be complete without at least mentioning the importance of key factors that actually shaped this organization. In this regard, it may be argued that many Latin American countries that have attempted economic or political integration have had little in common with each other. Argentina, Brazil, Chile, and Uruguay, on the other hand, seem unusually complementary to each other, politically, economically and historically. They have many things in common, starting with geography: three out of the four (Paraguay / Argentina / Brazil) share extensive common borders, as do Brazil and Uruguay. Uruguay and Argentina were once even politically united as they were part of the United Provinces of the River plate from 1810 through to $1880^{3}$. The political systems of all four countries had undergone recent substantial democratization and had taken part - as argued by Coffey (1998 pp257-9) - in the process of economic liberalization that took place in the region in the 1970's and 1980's. All four countries actually traded with each other, and had a history of economic cooperation, including joint administration of transnational infrastructure projects.

In spite of prior cooperation between them in the construction of Corpus and Ytaipu dams in the late 1970's, relations in the early 1980's between Argentina and Brazil turned out difficult. Argentine military governments tended to foster rivalry with Brazil and their foreign policy towards that country leant toward stressing competition rather than supporting cooperation. Brazilian governments, on the other side, responded with strong protectionist measures aimed at Argentine exports, whose quantity and value had fallen greatly since 1980 (Alterini \& Boldorini, 1995). This situation changed after the military regimes of Argentina and Brazil were replaced by democratic governments by the mid 1980’s.

Both the Brazilian and Argentine governments began to see the advantages of further cooperation and integration which led to the signing of a number of agreements in quick succession. Coffey (1998) further argues that this turn of events was in fact unavoidable and that the relationship between Argentina and Brazil could be described as similar to that of France and Germany at the time of the creation of the European Economic Community: both countries had a long history of inter-relationships, sometimes hostile, and the exports of one (France, or in this case Argentina)

\footnotetext{
${ }^{3}$ See - information available at http://www.wtove2003/com/ing_argentina.shtml
} 
tended to be mostly agricultural, while those of the other (Germany, or in this case, Brazil) tended to be primarily industrial.

Economic data extracted from the CIA Factbook (2002) suggests that this degree of economic complement seems to validate the existence of the common market. Brazil and Argentina are South America's largest countries, both in territory and population, each having large and developed consumer markets. Argentina is rich in natural resources, and counts on a highly developed export-oriented agricultural sector - while its industrial sector is regarded to be weaker than other sectors, despite depending upon a strong and competitive export-oriented automobile industry. Its service sector is considered large, accounting for 66\% of the country's GDP in 2001. Its exports - estimated in USD 26.5 billion in 2000 - included edible oils, fuels and energy, grains, foodstuff, vehicles, and mining concentrates more recently. Its imports - which totaled USD 23.8 billion in 2000 - chiefly included machinery and equipment, motor vehicles, chemicals, metal manufactures, and plastics. Argentina’s main foreign partner was Brazil, followed by the United States, Chile, Spain, China, and Germany.

Brazil, on the other hand is a much larger economy than Argentina, has well developed agricultural, mining, manufacturing and service sectors. Its main manufacture exports are textiles, footwear, chemicals, cement, lumber, iron ore, steel, aircraft, motor vehicles and machinery. Its export value in 2001 was USD 57.8 billions and it imported USD 57.7 billion. Argentina was Brazil's second most important trading partner in 2001, directly after the USA and ahead of Germany, Japan, Italy and the Netherlands.

Other MERCOSUR members, Uruguay and Paraguay, have different strengths that the bigger members do not, namely a very well developed financial and service sector in the case of Uruguay, and an export-oriented energy producer in the case of Paraguay. Even though there are a number of segments where all countries overlap and compete with one another, it becomes apparent that complementation works as a uniting factor and pushes the system toward further integration. Thus, Brazil’s extensive manufacturing sector has a natural market among Argentina’s, Uruguay's and Paraguay’s populations. 
Argentina's extensive natural resources and agricultural sectors also have ready made markets in Brazil. Uruguay has had extensive economic connections with Argentina with particular emphasis on the financial sector, while Paraguay's electrical generation industry very profitably supplements Argentina's and Brazil's massive energy needs. It can be argued that these economic connections and long history of interaction prepared the ground for better understanding and communication among the members. This, at a later stage, would bring about the creation of the MERCOSUR Atlantic Corridor Project as a way to face the ever-changing economic trends that the globalization process operates throughout the region. It is difficult to imagine a Corridor Project prospering without having its members, institutions, companies, and people go through some sort of economic and historical evolution where both historical and emotional ties influenced practical issues that needed to be resolved for the block to trade and prosper in a cooperative venture that influenced the ever-deepening relationships. Some analysis of logisticrelated economic information has to be first and briefly introduced before proceeding to the main concept of this thesis.

The MERCOSUR common market was established to accomplish the following goals:

* Elimination of tariff and non-tariff barriers;

* Adoption of a policy for a common external tariff (CET);

* Coordination of macroeconomic and sector trading policies; and

* Member-country commitment to the free movement of services, labor, and capital.

The MERCOSUR functions within the greater frameworks of the Latin American Integration Association (ALADI) and GATT (General Agreement on Trades and Tariffs), which permit members to provide preferential treatment within customs unions, while prohibiting additional tariffs to be levied on outside countries. The trade opening-up program focused on eliminating customs rights on foreign trade and prohibit member countries from unilaterally impeding mutual trade by targeting an end of duty requirements and non-tariff restrictions.

MERCOSUR's Atlantic coast stretches 3,500 miles along eastern South America, and the combined geographic area of more than 4.5 million square miles is considerably larger than that of the United States. It is the fourth largest integrated market in the world after NAFTA (North- 
American Free Trade Agreement), the European Union, and Japan. The distribution of population throughout South America's territory is not homogeneous. A large portion of it is concentrated over the coastal zones, while its central part exhibits a low demographic density below one inhabitant/square kilometer. The total population is approximately 350 millions, about $60 \%$ belonging to the MERCOSUR countries and predominantly located along its Atlantic seaboard over 5,000 kilometers. Vivacqua \& Stehling (1997) state that the MERCOSUR economy is large, with a GNP close to one trillion USD, while its output concentration is a factor that should also be considered.

In the case of Brazil, its major industrial and urban centers are located within a coastal stretch roughly 600 kilometers wide. In the case of Argentina, its major industrial output and urban settlements are located within the Buenos Aires province with the port of Buenos Aires as its main gateway to the world. To conclude, three sub-regions can be identified along the Atlantic seaboard, which contains the bulk of the MERCOSUR economy:

- The north-northeast of Brazil with a population close to 50 millions and a GNP over USD 100 billions/year;

- The south-southeast of Brazil, containing a population of more than 80 million generating over USD 700 billions per year; and

- The River Plate region comprising the great Buenos Aires, Montevideo and Asuncion areas, generating a GNP over USD 200 billions/year.

It becomes apparent that there are great concentrations of both supply and demand levels in these areas that are well identified and geographically isolated but they show little physical connection such as being linked by road, rail etc. Given the long distances between these concentrated areas of potential inter-area trade, one would expect the opposite- -higher physical links. The MERCOSUR's size along with the existing long distances between its various industrial and urban poles generates sizable transportation flows over long distances. Additionally, the need for the region to accomplish larger transportation economies of scale to therefore increase its negotiating power with other world blocs makes logistical integration a key tool toward fulfilling this need. Building a suitable logistic system seems to be a key enabler towards meeting the 
needs and goals of the MERCOSUR. The next section will introduce the reader to the main concepts of the Corridor as an intent to explicit the macro-ideology upon which same is based.

In this way the MERCOSUR concept can be seen not merely as a political and economic initiative, but rather as a radical change vision being translated into a mission and goals to be realized through a comprehensive and lengthy program of change and logistics projects.

\section{3 - MERCOSUR ATLANTIC CORRIDOR: A MACRO APPROACH}

On September 1st, 2000, the Summit of the Presidents of South America released a communiqué emphasizing the importance of counting on an integrated infrastructure (transportation / energy / telecommunications). The communiqué directly addressed the continent's transportation infrastructure envisaging the optimum use of different modes (land / air / maritime / inland waterways) in order to facilitate the border traffic of people, vehicles, and cargo. The presidents called for an infrastructure integration plan to be developed by the Working Group on Bi-oceanic Land Corridors. In essence, this demonstrated a significant emphasis of the first South American summit placing on trade and development of transportation infrastructure along the region's prevailing corridors (Agencia Brasil 2000). Appendix “C” displays the Protocol of Intentions for the Corridor and gives the reader a good general idea of its aims.

The trade corridor in South America emerged as an important agent of economic development with transportation being its fundamental subsidiary component. Despite the tendency to restructure the state to become more normative and assume a regulatory posture, it may be stated that Latin American governments in general, are still pressing forward to invest in transportation infrastructure. Though the Summit did address and identify strategic infrastructure needs, in reality the investment capacity keeps being heavily targeted toward highway construction and not toward more integrative and cost-reducing maritime, inland waterway, and intermodal transportation. In this regard Vivacqua and Stehling (1997 pp4-6) stated that trade among MERCOSUR partners had been increasing rapidly_from USD 5 billions a year from 1995 to a projected 15 billions in 2000. Land transportation generally prevails despite the very long 
distances between countries ${ }^{4}$. In the case of Brazil, the largest economy in the region, land transportation accounts for more than $70 \%$ of the total transported volume within Brazil.

Vivacqua and Stehling (1997) further contend that Brazil’s internal transportation costs rank among the highest in the world — 60\% above the USA, twice that of Canada. Furthermore, most cargoes in and out the northeastern region in Brazil, are carried by truck to and from the River Plate which lies 5,000 kilometers away. At this point an obvious question can be posed reflecting on one of the many challenges the Corridor has to face. Why is coastal navigation, an intrinsically more economical mode of transportation, not utilized in large scale alongside the Atlantic coast of South America? This question is especially relevant when all the physical and geographical elements for such development seem to be in place-a large expanding economy, an ever-growing population distributed along the coast, and large volumes of cargo to be carried..

The answer could be that since the 1950s the two larger economies of the region - Brazil and Argentina - favored highway transportation to consolidate their respective development of a strong automotive industry. Notwithstanding the above, the consortium of the MERCOSUR Atlantic Corridor resumed the strategic vision of making the existing coastal and inland waterway transport systems more competitive and integrating them with other transportation modes and infrastructural nodes.

Panama is one hypothetical hub that might well replicate the Corridor's main integrative function. This initiative aimed to expedite and facilitate logistics integration among the MERCOSUR countries while taking advantage of the privatization program of port facilities, and the ever-expanding investment in terminals and transfer equipment and facilities. The master goal, without prejudice to a number of other goals that emerged through the process, has been the transfer of some tens of million tons of cargo from the intra-coastal highway network to a coastal navigation system capable of connecting to an inland waterway sub-system. Consequently, regional level decision-makers at the intervening ports decided that they should jointly develop a variety of process and change management initiatives to:

\footnotetext{
${ }^{4}$ With poorly paved and maintained road systems, land transport continues to be less efficient and effective than fluvial transport along the vast inland waterways
} 
- Lower operational costs, rationalizing the ship-to-shore interface, and contributing to enlarge regional economies of scale;

- Act locally as coordination centers between the local inland transportation network and coastal navigation through the organization of a local, integrated, multimodal transportation sub-system monitored by the port;

- Organize an operational association among the several ports along the coast to promote integrated door-to-door operations via coastal navigation and by linking their respective inland transportation suppliers;

- Act locally as a trade and investment promotion center; and

- Promote the creation of potential land-bridges linking the Atlantic with the Pacific across the member countries by optimizing all the available logistic means at regional level.

South America has been developing a unique style of overcoming the obstacles to trade presented by the existing imbalances of its transportation systems as a result of facing a legacy of un-integrated and non-standardized highway, railway, river, ocean, and coastal transport networks. It can be acknowledged that in the absence of a top-down comprehensive intermodal approach to regional transportation, public and private interests have coalesced in the form of integration roundtables to make transportation more efficient, respond to globalization trends, help enlarge economies of scale, enable alliances, partnerships and joint-ventures, and gain overall competitive advantage. The above mentioned integration roundtables are centered on a regional port and indicate an evolving conception on integrated port networks in Latin America analogous to the port networks in ascendancy in Europe (van Klink 1997).

It becomes apparent that through its integration roundtable ${ }^{5}$ model, the MERCOSUR Atlantic Corridor approach has emerged as a regionally endogenous form of problem solving currently spanning several South American countries. Moreover, this model seems easily transferable to other regions in the world where considerable imbalances between production and consumption poles or between efficient and inefficient transportation systems and infrastructural nodes exist. If these imbalances are translated into large cargo flows that can be rationalized and optimized (by

\footnotetext{
${ }^{5}$ A form of community of practice (CoP) that will be explained in more detail in Chapter 3
} 
cooperating, exchanging knowledge, becoming regionally organized, and/or facilitating trade and investment through enlarging economies of scale and gaining competitive advantage) then the model may become a very powerful tool. Replication of this model might then be useful to stimulate trade growth and development, overcoming structural obstacles to enlarged economies of scale and gaining competitive advantage by narrowing existing technological, operational, and managerial gaps between countries and regions.

This model may possibly be applied to the following regions and countries where there are a number of imbalances in available facilities, technology and management:

- South-central USA and the region comprised between Central America and the Caribbean where two common markets work independently and remarkable gaps are evident (CAFTA: Central America Free Trade Agreement; CARICOM: Caribbean Community). A difficult geography, lack of infrastructure, high level of obsolescence, and great cultural diversity are just a few aspects that can be overcome to achieve greater economies of scale and competitive advantage.

- Australia-New Zealand and the region made up of Indonesia, Philippines, and Southeast Asia. This is a vast and densely populated area where technological, managerial, and cultural gaps become apparent, whereas it holds a tremendous growth potential. Like the previous example, geography is extremely complex, there is ground for a considerable upgrade in terms of logistics infra and supra-structure, distances are even more important, and culture plays even a more important role if well understood and managed toward accomplishing trade enlargement and generating efficiencies.

- Western Mediterranean countries and the northern Africa region - where not only ocean shipping and ports turn out to be pivotal for trade development, but also the design and setting of land-bridges that might help reaching the heart of both continents by making a strategic use of ground logistics and air logistics. Here, similar to the previous example, effective transfer of successful models and managerial experiences across Europe, might result in enlarged economies of scale and betterment of overall competitiveness of the entire trading bloc. 
There are many regions and sub-regions across the globe where structural gaps and considerable growth potential may be identified. The above - as well as many other possible examples presupposes that the intervening parties have a strategic interest along with their understanding of the long term implications arising out of the decision-making process. Geopolitical interests may also play a key role here too but this aspect is not the focus of this thesis.

\section{4 - MERCOSUR ATLANTIC CORRIDOR: A MICRO APPROACH}

The MERCOSUR Atlantic Corridor comprises an organization that gathers a significant number of companies which hold regular meetings where their respective problems are made public before the participating members. These problems and issues are discussed, assessed, while potential solutions are proposed, examined, and tested in the respective field.

The scope of the organization spans across the MERCOSUR and participants come from a wide range of activities — such as logistic operators, ocean and river port operators, railway companies and operators, barge and tugboat operators, trucking companies, port services firms, warehousing and distribution companies, air-cargo operators and airport terminals, customs brokers and national customs authorities, shipyards, banking, insurers, exporters, importers, traders, shipbrokers, government, education, legal firms, environmental consultancy companies, etc .

Meetings are held once a fortnight. Information sharing amongst participants is conducted through an on-line intranet system that is accessed through what is called integration roundtables to become explicit, whereas knowledge relating to comments and conclusions are later distributed in a face-to-face mode. These on-line forums link a growing number of cities and ports throughout the MERCOSUR countries. They integrate them into a node where regional discussion on operational and commercial issues affecting the growth of business takes place, in tone with the on-going globalization process. The main goal of these meetings is to find solutions or alternative ways to address the many problems encountered in the MERCOSUR region as a way to increase trade by sharing and transferring knowledge, and making regional supply and demand centers getting closer and meet their needs and potential in a more efficient manner. 
A major advance in the consortium concept emerged with the creation of cross-national roundtables established in Argentina, Uruguay, and Brazil, to later expand into Paraguay, Bolivia and Peru while discussions continue to be held to let other countries join the system - either regional or extra-regional ones. Thus a truly continental organizational economic development model is expanding based on the assembly of key actors involved in the logistic process with the goal of reducing operational bottlenecks and costs while increasing trade opportunities and fostering strategic alliances at the same time.

The integration roundtables are normally established at a port community and fall under the responsibility of a "regional coordinator", who carries out the following functions:

- Continuously expanding the Corridor's membership and the regional network of affiliated roundtables;

- Calling the roundtable meetings and acting as a motivator so that the expected results are accomplished;

- Continuously stimulating the exchange with other roundtables in their multiple forms - teleconferences; business road shows, reciprocal consulting; intranet;

- Continuously stimulating the affiliation of companies to the data bank, with special concern with its updating, as well as its use by third parties; and

- Developing proposals and contracts offering services on regional and business promotion and consultancy on matters of commerce and logistics.

Meetings can often promptly resolve a given problem just by assembling the necessary actors. In the case of larger, more fundamental or complex economic development issues involving larger regional areas or economic interests, the roundtables can initiate the process of dialogue and, with staff resources, mediate and facilitate data and information exchange in search for potential alternative solutions.

An illustration that can be provided is that of the meeting originating in Vitória, the leading port and capital of the Brazilian state of Espírito Santo, where the MERCOSUR Atlantic Corridor's focus lies on generating regional development. To do so, it was necessary to convene integration roundtable meetings that included leading players in the region's basic industries, warehousing 
companies, transportation services, governments, custom houses, labor, media, shippers and many others. The purpose was to resolve a wide array of issues. Examples of these issues include port congestion, ship-to-shore transfer imbalances, interruptions in the cold-chain for reefer containers, or an increasing rate of transportation-related claims. This illustrates how the roundtable meeting can help create the conditions for companies to interact, exchange data, produce information and knowledge and therefore help solve problems affecting key players through promoting this form of corporate socialization. This process was expected to generate added value and perhaps most importantly, a point of cooperative behavior could be reached, to result in potential strategic alliances, partnerships, alliances, mergers or even acquisition among firms in gaining competitive advantage and facing whatever peril or change that the globalization wave may bring in terms of technology, innovation, competitiveness, and scale of business.

The specific micro-objectives of the Corridor can be summarized as follows to:

- Create a favorable space for the development of integrated logistic systems under the concept of door-to-door logistics;

- Stimulate the participation on the basis of a win-to-win system, where all the participants have something to win from their presence;

- Attract a growing number of ground transportation companies, railway companies and operators, ocean shipping firms, ocean and river port terminal operators, shipping agencies, warehousing and distribution companies, exporters and importers, unions, universities, authorities, etc.;

- Create innovative logistic systems leading to achieve greater productivity and larger trade economies of scale;

- Solve explicit problems and remove obstacles through formulating and implementing solutions to those specific problems, and fostering partnerships and alliances among participants; and

- Transforming ports and its associated communities into logistic integration nodes between its natural hinterland and the members of the Corridor.

The MERCOSUR Atlantic Corridor has currently only one similar organizational entity, that is similar in being a geographic extension of a region but with a limited or modest objective. The 
Northern Maritime Corridor (NMC) takes place in the north Atlantic by gathering eight maritime nations together aiming to improve a number of issues that they commonly face. The NMC is the chosen term for a sea based transportation corridor that stretches from Northern Norway and North West Russia to the Continent, connecting the coastal areas of the North Sea and the Northern Periphery; 20 regions in eight countries (Germany, The Netherlands, Flanders, Scotland, The Faeroe Islands, Iceland, Greenland, Sweden and Norway). The vision of the NMC as stated on its web site ${ }^{6}$ is to provide: "A means of efficient, safe and sustainable transportation, connecting coastal areas and enhancing regional development in the North Sea Region and the Northern Periphery Area”. The vision suggests a more focused objective which becomes apparent when coming to examine what its goals are:

$>$ Promote the short-sea-shipping links;

$>$ Improve seafood transport and logistics;

$>$ Enhance the maritime transport in the petroleum sector; and

$>$ Betterment of maritime safety.

These goals suggest a much more concentrated list of objectives with a much smaller scope of action; however, it also highlights the importance of two aspects this thesis will focus on: networking as a fertile ground to enhance knowledge sharing and transfer through the participation of both public and private actors. The main difference between the NMC and the MERCOSUR Corridor is perhaps given by the stress the NMC places on the region to region cooperation concept, vis-à-vis a supra-regional and almost continental approach on the MERCOSUR.

Figure 2.1 shows the large geographical scope of action of the NMC which despite involving eight nations, only concentrates on functional targets whereas conducting a regional approach, these being the main differences with the MERCOSUR Atlantic Corridor. Such a limited functional scope of action suggests that being maritime nations and counting on a higher relative operational and technological development than the existing in the MERCOSUR Corridor, their needs differ from the latter while a region-to-region approach makes more sense on a given

\footnotetext{
${ }^{6}$ See URL http://www.irogaland.no/ir/public/openIndex/view/list_nmc2006.html?ARTICLE_ID=1140187268312 accessed April $5^{\text {th }} 2009$.
} 
number of segments - and not a supra-national treatment where major imbalances are nonexisting.

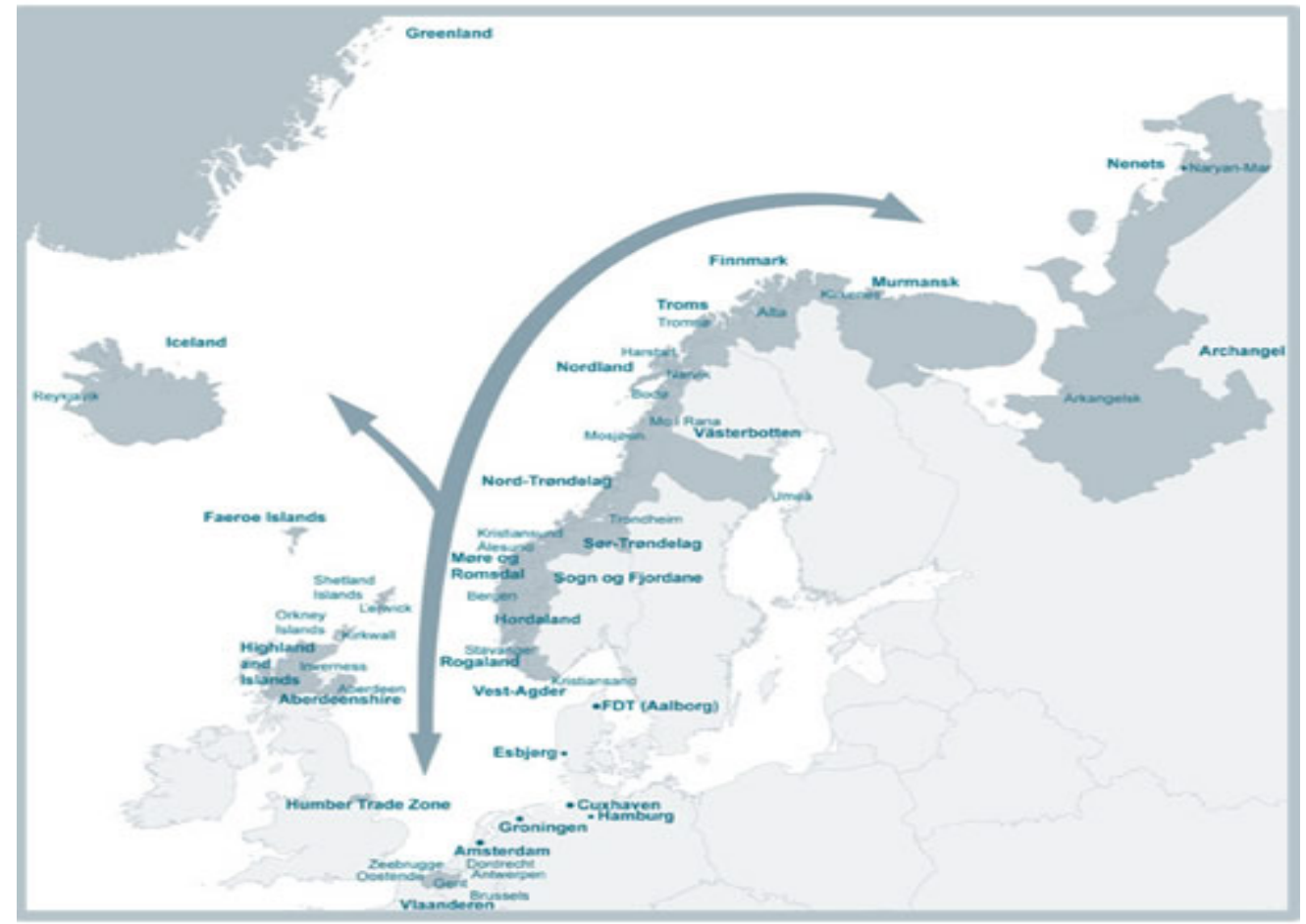

Figure 2.1 : NMC's geographical scope

This example turns out useful to determine one, and possibly the most important, pre-condition that justifies a more ambitious corridor such as the MERCOSUR addressing development imbalances between sectors, regions, and countries.

\section{5 - INTEGRATING ROUNDTABLE CONFIGURATION EXAMPLE}

This section is presented in two subsections. Sub-section 2.5.1 relates to a justification of supporting the view taken of the roundtable discussion infrastructure as one, supported by the Corridor's IT and organizational infrastructure, can be viewed as a form of strategic community of practice CoP followed by sub-section 2.5.2 which explains how the Corridor performs as a kind of strategic CoP. Wenger McDermott and Snyder (2002) describe a CoP as an organizational form where a group of people join together to pursue a common interest. 


\subsection{1 - Justification for the Corridor as an Entity Facilitating Strategic CoPs.}

By definition, a community of practice (CoP) is a natural part of organizational life and they are expected to develop through their own initiative, whether the organization recognizes them or not. Even though it is not the intention to develop the discussion of this type of entity much further at this point, it is important to highlight that a CoP's sustainability depends, to a great extent, on the voluntary engagement of their members and on the emergence of internal leadership - informality and autonomy being fundamental components of the system.

However, and as it will be exposed throughout this dissertation, this has a direct link with both the context these CoP were intended to established and consolidated and the cultural dimension of the various participating regions and countries. It has been the author's experience as a direct participant that even though there is a voluntary component in any CoP, there is also a driving element that is determined by the particulars of the business environment it operates. Geographical differences and sector particulars by industry became apparent, as well as idiosyncrasy and business contextual gaps.

My role during the early years of the Corridor project was to recruit new members around South America's south cone countries, as well as identify potentially new places to establish additional roundtables that help link production and consumption poles in a more efficient way. Identifying vulnerable sectors as well as targeting realistic reengineering possibilities across the region, was only part of the task. Making these regional players aware of the upcoming globalization impact, especially those far located from the main industrial poles, turned out to be perhaps the most difficult part in that old regional rivalries and on-going distrust had to be somehow overcome.

Such a role was carried out by fulfilling certain parameters in terms of budgeting priorities and a very specific time framework - both variables being traditional PM processes. This role allowed me to appreciate a number of different PM related realities that introduced complexity of undertaking PM processes across a vast geography, as well as to conceptualize the nature of the existing priorities as well as to identify potential intra-sector synergistic opportunities. 
Wenger et al. (2002, pp6-7) argue that success in global markets depends on communities sharing knowledge across the globe. Naturally, in order to maintain its global character a varied array of features such as culture, leadership, geography, and religion - among many others and further to knowledge sharing and transfer itself - had to be considered by those corporations that decided to join the Corridor as an alternative to face the future. The Corridor did not look for global success but that it was set up as a reaction to the global threat of world scale newcomers holding much larger economies of scale and an almost endless resource base.

Pettigrew (1997) suggests that when comparing organizations from different sectors, issues of industry context must not be overlooked. Firstly, any investigation or comparison between managerial processes in different sectors must consider the political, economic, social, technological, legal, environmental and structural factors inherent to each sector. Secondly, an organization itself is historically the product of its own politics, economics and social factors that contribute to the use of processes, practices and philosophies. It is precisely this understanding that leads Pettigrew to suggest an outer and inner context that collectively helps to determine the feature of a practice. These features adopt very different and dynamic forms when both a regional context and an ever-fluctuating business environment is considered from a knowledge sharingtransfer and CoP perspectives with respect to Latin American countries.

Wenger et al (2002, pp12-14) further argue that some CoPs grow spontaneously whereas others may require careful seeding and further pushing and close follow-up. In this regard it is an accepted opinion that organizations can do a lot to create an environment into which they can prosper by encouraging participation, removing barriers, assigning resources, and giving them a say in the decision-making process (Storck and Hill, 2000 pp63-74).

Walker and Christenson (2005, pp287) provide a useful comparison of the features a community of interest, CoP, project Team, Project management Office (PMO), Project support office (PSO), Corporate management office (CPO), and Centre of Excellence (CoE). These terms used are mainly defined and discussed later-readers may refer to Section 3.9 for project office forms. They use the three dimensions of social capital offered by Nahapiet and Ghoshal (1998) of structural, relationship and cognitive dimensions. The 
structural dimension refers to the strength, configuration and nature of ties between individuals and groups. The Relationship dimension refers to trust, norms and cultural aspects later elaborated upon in Section 3.8. The cognitive dimension relates to shared understandings, through shared codes and language (technical or national) also discussed further in Section 3.7 and Section 3.8.

\begin{tabular}{|c|c|c|c|c|c|c|c|c|}
\hline $\begin{array}{l}\text { Knowledge network } \\
\text { type }\end{array}$ & $\begin{array}{l}\text { Structural } \\
\text { dimension }\end{array}$ & $\begin{array}{l}\text { Relational } \\
\text { dimension }\end{array}$ & $\begin{array}{l}\text { Cognitive } \\
\text { dimension }\end{array}$ & $\begin{array}{l}\text { Dominant } \\
\text { learning } \\
\text { level }\end{array}$ & $\begin{array}{l}\text { Degree of } \\
\text { hierarchy }\end{array}$ & $\begin{array}{l}\text { Organisational } \\
\text { knowledge } \\
\text { retention }\end{array}$ & $\begin{array}{l}\text { Level of potential } \\
\text { wisdom generation }\end{array}$ & $\begin{array}{l}\text { Corporate support } \\
\text { resources required }\end{array}$ \\
\hline $\begin{array}{l}\text { Community of interest } \\
\text { (CoI) }\end{array}$ & No & Yes & Maybe & $\mathbb{L}^{a}$ & Very low & Very low & Low-medium & Nil \\
\hline $\begin{array}{l}\text { Community of practice } \\
(\mathrm{CoP})\end{array}$ & Yes/maybe & Yes & Yes & $\mathbb{L}^{2} / \mathrm{GL}$ & Low & Low/medium & Medium-high & Nil-moderate \\
\hline Project team & Yes & Yes & Yes & $\mathbb{L} / G^{a}$ & High & Low/medium & Low & Moderate \\
\hline $\begin{array}{l}\text { Project management } \\
\text { office (PMO) }\end{array}$ & Yes & Yes & Yes & $\mathbb{L} / \mathrm{GL}^{\mathrm{a}}$ & High & Low/medium & Low & Moderate \\
\hline $\begin{array}{l}\text { Project support office } \\
\text { (PSO) }\end{array}$ & Yes & Yes & Yes & $\mathbb{L} / G^{\mathrm{a}}$ & High & High & Medium & High \\
\hline $\begin{array}{l}\text { Corporate project } \\
\text { management office } \\
\text { (CPO) }\end{array}$ & Yes & Yes & Yes & IL/GL $/ \mathrm{OL}$ & Very high & Very high & Medium-high & Very high \\
\hline $\begin{array}{l}\text { Centre of excellence } \\
(\mathrm{CoE})\end{array}$ & Yes & Yes & Yes & $\mathbb{L} / \mathrm{GL} \mathrm{OL}^{\mathrm{a}}$ & Very high & Very high & High & Very high \\
\hline
\end{tabular}

Figure 2-2: Forms of Knowledge Networks

Figure 2.2 can be viewed in light of the Corridor. It has some features that suggest that the Corridor could be considered a form of PMO as is later argued in Section 3.9. However, Figure 2.2 illustrates, supported by the context descriptions of the Corridor and further discussion on the literature of CoPs offered in Section 3.7, that the roundtable discussions facilitated by the Corridor can be primarily seen as a CoP. Structural, relational and cognitive dimensions clearly support this being in agreement with Figure 2.2.There was evidence found in this thesis of both individual and group learning as members brought back knowledge acquired into their organizations. Organizational learning within the Corridor itself as well as participant organizations may be described as high because of the continued refinement and improvement gained through experience. In terms of wisdom, which Walker and Christensson (2005, pp276) refer to as 'the judicious choice of alternatives', there is plenty of evidence that will be presented in the analysis of Corridor initiative case studies presented in Chapter 8 that supports the Corridor is a form of CoP. The last column of Figure 2.2 shows corporate support resources required and in this case the Corridor required active participation and people contact so it could be seen to be medium to high. Nevertheless, this provides adequate justification to view the Corridor 
roundtable discussions as being a form of $\mathrm{CoP}$ and that the Corridor itself holds many attributes shared by various forms of PMOs facilitating CoPs.

Probably one of the tallest barriers to overcome, not only in South America but in the entire Latin America region, turns out to be of cultural nature and is linked to an existing and growing degree of skepticism. This is due to formulas or techniques that have been successfully applied in distant environments being promoted but where both macro and micro variables (the context) show very little similarities with those at regional level. Added to this was the existing historical mistrust and rivalry between nations in the region.

This has been my experience when attending the integration roundtables, where speakers have proposed foreign examples as potential models to be implemented or adapted to the regional reality, when these proposals have been categorically rejected due to existing practicality gaps in terms of business environments, cultural aspects, and the general perception that the upcoming economies of scales and technology of the global firms were potential threats. This can be contrasted with the opportunities to grow, adapt, and even expand by reengineering structures, rationalizing their operating assets, or engaging in some type of vertical integration process.

In general, it may be affirmed that the urgency of short run results within the MERCOSUR region attracted a great part, if not all, the attention of the corporations at the expense of longer term issues of possibly much more strategic value. From this viewpoint, and according to the author's experience, these CoPs had to go through difficult times and often needed permanent back-up and follow-up support to divert their members' attention from the burning daily problems of keeping their attention focused on the subject matter a given CoP was working with.

The CoP concept in not new, neither is the need for organizations to become more intentional and systematic about managing knowledge in an ever-changing business environment. However, it becomes apparent that companies operating in world markets need to make good use of people who stand out in their CoP, no matter where they come from, meaning that corporations no longer struggle for market share only, but that they also compete for talent. 
Cappelli (2000, p103) argues that in terms of talent and from a retaining talent perspective, professional relationships and social ties are important reasons why people stay with a company. Cohen and Prusak (2001, p19) argue that companies can win the war for talent by offering employees the opportunity to build a sense of community with workplace colleagues. One of the Corridor's objectives relates to talent creation as a way to let innovation and change emerge in a number of industry segments that made their living in a highly restrictive market context.

Hamel and Prahalad (1994, pp56-58) discuss genetic reengineering as another way to introduce more genetic variety into a population from a talent recruitment viewpoint, aiming at bringing in new members who cross-breed with the old and alter the existing genetic pool. Also relevant here, Dixon (2000, p11) states that “The common knowledge that is generated internally, by talented employees in the act of accomplishing the organization's task in new and innovative ways, is where knowledge sharing can really pay off”.

It is often argued that the knowledge of experts is an accumulation of experience, (a kind of "residue" of their actions, thinking, and conversations) that remains a dynamic part of their ongoing experience (McDermott 1999, pp103-117). Therefore this type of knowledge, which is much more a living process than a static body of information, forms an integral part of a CoP's activities and interactions further to serving as a living repository for that knowledge.

Talent seems to be a fundamental component and CoPs turn out being useful tools to retain talented employees for the benefit of the organization. However, it also poses a strategic obligation on the part of the corporation to regularly supply challenging targets to keep talent busy while intending gain competitive advantage. This is a topic where this dissertation intends to deepen the existing literature, with particular emphasis on the way CoPs contributed to sharing, creating, and transferring different knowledge. The development of these CoPs became, step by step, challenging projects for both the companies and their talented employees to address.

A CoP should not only be a helpful tool to generate new ideas and drive innovation but also create an alerting system with respect to the probably various defective genes an organization should get rid of before launching into new challenging strategic paths. This transformational 
perspective of the Corridor proved useful as the new business context approached the region. Such an alerting mechanism tended to identify where talent was missing or had deteriorated.

Fernie, Green, Weller and Newcombe (2003, p180) are of the opinion that strong ties, identified by high-trust is of paramount importance in project management alliances and joint-ventures. Lengthy timeframes and close relationships are ideal for the sharing complex tacit knowledge. Weak ties, however, limit the exchange of knowledge and even information, as is clearly exposed within the Corridor project. However, it is also conceded that the knowledge created by highly socialized or strongly tied groups is unlikely to be innovative or supportive of talent emergeing this suggests that CoPs are likely to produce better results if they find themselves somewhere in the middle and far from the extremes poles of being high trust / no trust, or strong ties / no ties.

\subsection{2 - The Corridor Performing as a Strategic CoP}

Given the special characteristics of the Corridor and the heterogeneous membership of the roundtables, it turns out interesting to highlight that even though trust and socialization were variables in permanent evolution, their members rarely forgot that they were actually competitors and therefore tended to keep a certain strategic reserve of information in a defensive fashionthough probably they were compelled by the uncertainty generated at the outset of the project by the business environment. Further to the founding firms (PETROBRAS / CVRD / BND), the Corridor embraces a growing number of members comprising a wide spectrum of activities and regions:

$>$ LIBRA DO NAVEGACAO, Santos Shipping company.

SADIA, Sao Paulo Foodstuff industry.

$>$ VOLKSWAGEN, Buenos Aires .Automotive industry.

$>$ PORTO DE SAO SEBASTIAO, Sao Sebastiao. Container Terminal.

$>$ INFRAERO, Sao Paulo Airport Authority.

$>$ DOCENAVE, Rio do Janeiro Shipping company.

$>$ C.P. DE BAHIA BLANCA, Bahia Blanca..................Ocean bulk Terminal

$>$ PUERTO MADRYN, Port Madryn....................... Ocean mutipurrpose.

$>$ FIESP, Sao Paulo......................................SME Chamber.

$>$ ABIA, Sao Paulo.......................................Food Chamber. 
$>$ MERCOSUR TRADE CENTER, Buenos Aires. Trading company.

> IMPSA, Mendoza. ..Industrial firm.

> Maruba, Buenos Aires Shipping company.

> TRANSROLL NAVEGACAO, Santos .Coastal Feeding co.

$>$ CSAV, Santiago Shipping company.

ALL, Sao Paulo .Railway company.

$>$ NUEVO CENTRAL ARGENTINO, Cordoba. Railway company.

$>$ BELGRANO CARGAS, Buenos Aires Railway company.

$>$ FERROSUR ROCA, Olavarria Railway company.

$>$ SIDERAR, Campana Steel company.

> URUCUM MINERACAO, Ladario. Mining company.

$>$ INTERSINDICAL PORTUARIA, Santos Port union.

$>$ TECON SALVADOR, Salvador Container Terminal.

$>$ TRP, Buenos Aires. Container Terminal.

$>$ ROSARIO PORT TERMINAL .River bulk termnal.

$>$ NUEVA PALMIRA PORT, Nueva Palmira. .River bula Terminal

$>$ TANGO LOGISTICS, Curitiba. .Ground transportation

$>$ TERMINAL 7, Buenos Aires .Empty container yard

$>$ ATZ, Zarate River auto Terminal.

$>$ PUERTO DESEADO, Patagonia... .Seafood Terminal.

> COMODORO RIVAVADIA, Patagonia Ocean oil terminal.

The integration roundtables were born in Brazil. Two pioneering engineers had foreseen that the upcoming global trends required extreme changes to allow the region's businesses to preserve their businesses within national and regional owners instead of selling off assets with no effective reaction on their part to counter that threat. The project was born in the Brazilian city of Vitoria, to later expand into the costal ports of Brazil, and inland cities of strategic importance within that country, in terms of output and consumption scales. Later, numerous other locations with a relative higher degree of logistic complexity proved to be of interest and these were embraced within the Corridor. 
A second stage emerged with the regionalization of the roundtables and the Corridor concept itself, by expanding the idea into Argentina and the other southern cone countries-Uruguay, Paraguay, Bolivia, and Chile. Later, Peru was added to the list of participating countries as its importance was recognized in terms of being a land-bridge to the Asia-Pacific Rim of high strategic profile for a number of massive cargo segments.

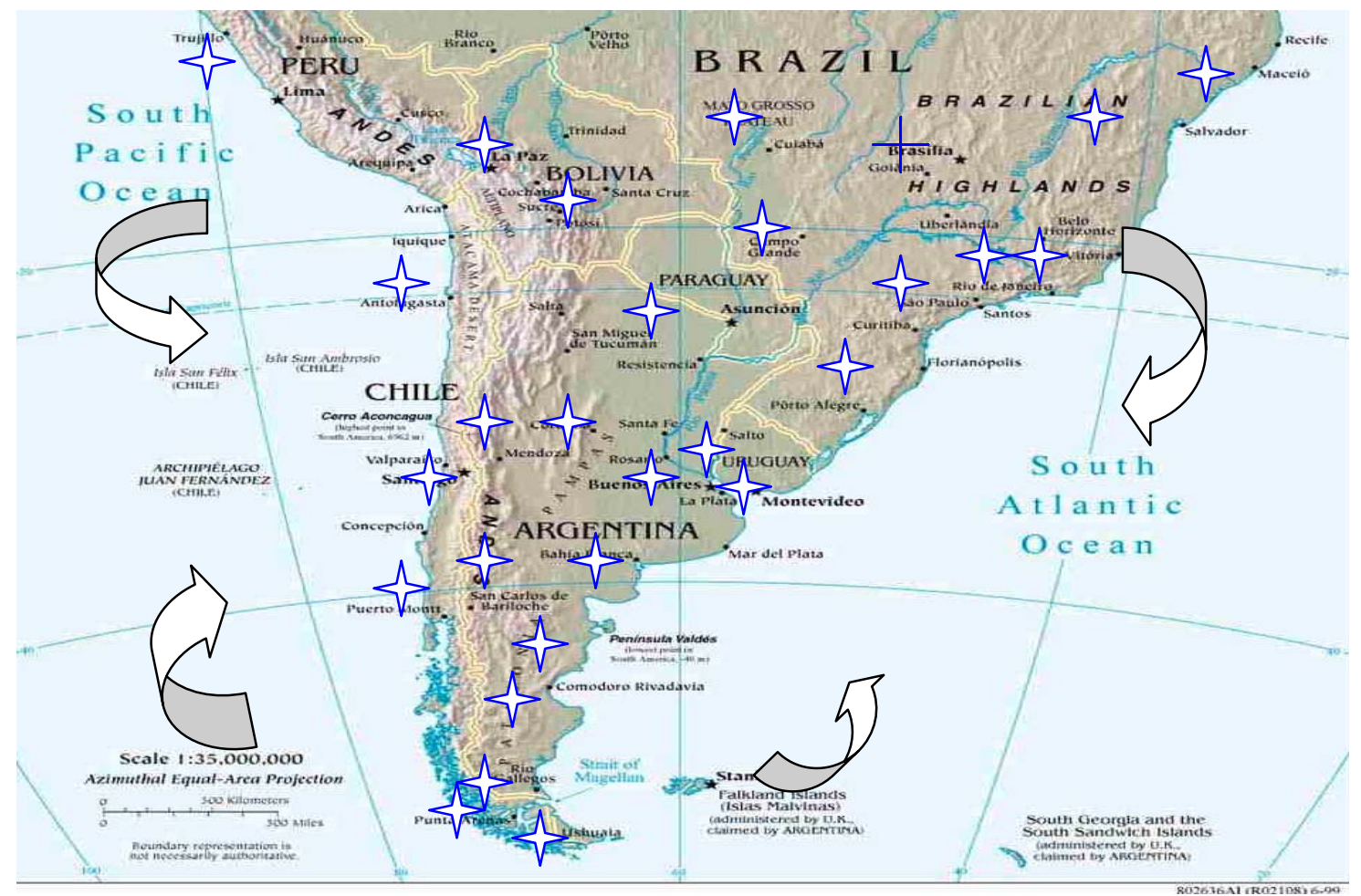

Figure 2.3: MERCOSUR Atlantic Corridor Consortium network; 2004.

Figure 2.3 illustrates the wide geographical area as well as the scale of the Corridor roundtable groups. These are represented by star symbols on the map.

Meetings are held regularly, are directed by a sort of manager or broker and its outcomes are made available via an intranet throughout the regional network as shown on Figure 2.3. These encounters can often resolve a given problem just by assembling the necessary actors in a single journey.

In the case of greater, more fundamental or complex economic or operational development issues, the roundtables can initiate the process of dialogue and, with staff resources, mediate and 
facilitate data and information exchange in search for potential alternative solutions by bringing together actors from both the public and private sectors.

Probably one of the roundtable's top priorities lies on adding value through the development of information databases capable in itself to generate trade leads and business development further to generating a fertile ground for potential investment currents. In this regard it is important to highlight that even though the roundtables have experienced a rapid expansion process leading to sustained value adding, it is precisely the high rate of growth that presents a possible weakness in terms of available technology to back up such an expansion.

Even though the Corridor rests on an intranet system which is regularly up-graded to cope with an ever-increasing demand, the spirit of this dissertation lies more on the concepts of knowledge sharing and transfer, leadership, strategy and CoPs, and not on the technology or information systems utilized to let knowledge sharing and transfer occur. No matter how well developed an intranet system may be as a device aimed to transfer knowledge; strategic or critical issues may turn state-of-the-art technological devices into a relatively valueless proposition should priorities not be well defined within the organization. The research in this dissertation finds that face-toface interaction among the Corridor's members was critical in making transformations happen, whereas technology seems to be restricted to an enabler or enhancer for this to happen.

Examples of knowledge management activities include the search of solutions to better handling hazardous chemicals or perishable goods in Brazilian terminals; harmonizing procedures for a smooth railways interconnection between Argentina and Brazil; upgrading aids to river navigation as to make night operations safe to reach Bolivian terminals; standardizing air-cargo terminal handling fares across the region; adopting similar regional criteria towards controlling and surveying ships flying flags of convenience; easing customs clearance procedures in Argentina to allow transit cargoes to Chile to proceed faster.

Information technology (IT) plays an important role within the Corridor and its upgrade can be regarded as an on-going challenge to better capture the generated knowledge across the regional roundtable forums. However, a bigger challenge to the development of the roundtables remains in 
that a given number of issues were and are yet deemed to be strategic and therefore not subject to disclosure, sharing, or transfer. Competitive advantage is often regarded to be at stake if a given piece of information was made available for others to assess and make use of it for their own advantage (e.g. terminal handling charges for a given shipping joint-venture; bunker prices or place of supply for a given air-cargo freighting company; operational advantages given by a railway firm to a major global shipping company to distribute containers across the region; fixed ocean freight rate levels for major volume customers; reliable new-tonnage supplier; shipbuilding contract particulars; achieved load/unload rates at a given hub terminal; etc).

Section 2.5.1 provided a justification for viewing the Corridor's Roundtable discussion groups as CoPs that were supported by both IT, and more importantly leadership, within a project management environment or context for facilitating and participating in a major transformational change by supporting and helping the development of these CoPs.

\section{6 - CHAPTER SUMMARY}

This chapter examined the constituting elements of the MERCOSUR common market by referring to the Asuncion and Ouro Preto Treaties that gave birth to this organization. It followed by giving an historical perspective and a brief picture of its potentiality of development by highlighting the existing relatively high degree of complementation of the member economies as well as the apparent political willingness to pursue the previously set objectives.

The chapter continued by addressing the Corridor from a macro-perspective, through underlying its role as agent for economic development. It also stresses its importance as an active tool to overcome the existing imbalances between production and consumption poles within the MERCOSUR, further to proposing other possible scope of its application in the world. The chapter concluded with a section that also deals with the Corridor from a micro-perspective and addressed both the role and the way the integration roundtables work, and it explained its objectives and the way they interact with its potential outcomes. 


\section{LITERATURE REVIEW}

\section{1 - INTRODUCTION}

This chapter begins by setting the study's context with organizational competition strategy providing one of several relevant theoretical frameworks that help to make sense of and to be used to analyze data used in this research study. Figure 1-2 illustrated the logic behind the thesis in terms of research question and methods and the need for literatures accessed and discussed. The cluster of theoretical areas also was shown. Business transformation evolves from business strategy that responds to competitive forces and the role provided by the Corridor. Action that translates strategy into the realized result also involves leadership to create value and effect change and PM skills. Further, it requires knowledge exchange and so the theoretical areas of culture and knowledge management are relevant to enact change. This context affects corporate strategy adopted by Corridor participant firms and so a brief explanation of the various strategy schools as well as the way that Porter's (1985, 2001) five competitive forces and the way they have influenced the various logistic industry segments across the studied region is provided.

The chapter continuous by assessing the theory of the firm while trying to ascertain what is behind the apparent and ever-existing need global companies display for market expansion, and how that process impacts on the regional companies and national economies. The resource based view of the firm informs a dominant assumption behind this thesis. It also helps us better understand the impact of structural variables as well as the role that individual expectations play with respect to the limits to the growth of the firm.

The chapter then establishes differences between a transactional and transformational leadership style, as well as clearly establishing the main particulars and characteristics of leadership and management in a Corridor-like business environment. It introduces the reader to the concept of uncertainty by highlighting its importance in this project, and develops a discussion on strategy and its various implications within the Corridor project and its players. It also addresses theory relating to how organizations cooperate as a way for companies to gain control over and 
establishe a given market. This highlights the importance of both uncertainty and market interdependence from a cooperation perspective and within a South American context.

The chapter then continues with a section that addresses the role to be played by the knowledge management (KM) and how it links with the Corridor project. In doing so, it both describes the project and relates to different types of knowledge creation and knowledge transfer. It also highlights the concept and utilization of a CoP within the integration roundtables across the region.

Figure 1-2 in Section 1.6 illustrates the research approach and how the combination of reflection on practice, reflection in practice, and reflection on DPM courses led me to the theories presented in this chapter. Therefore, it makes sense to not only present a traditional literature review that stands apart from theory but to also indicate where reflection on the experience of being an active participant has relevance to theory presented. It is anticipated that this will help the reader to appreciate how theory and reflection shaped hypotheses or propositions that were later tested.

Finally, as PM is argued to play an important part in effecting transformational change, there is a section that identifies recognized project management tools and techniques. The chapter is then concluded with a chapter summary.

\section{2 - COMPETITIVE FORCES AND INDUSTRY STRUCTURE}

Courtney, Kirkland and Viguerie (1997) argue that in turbulent and rapidly changing circumstances, traditional strategic planning horizons are dangerously constricting and confining. They categorize four levels of uncertainty with corresponding strategic responses:

- Level 1, with a clear enough future can be planned for using the traditional strategic toolkit as described by the positioning school of thought (Mintzberg, Ahlstrand and Lampel, 1998, Chapter 4);

- Level 2, with alternative futures lends itself to concentrating on a few discrete outcomes that define the future using analytical tools game theory etc; 
- Level 3, comprises a range of possible outcomes but no natural scenarios where scenario planning can be use; and.

- Level 4 is in the realm of true ambiguity with no basis to forecast the future so the main tools used for planning would be emergent by drawing analogies, recognizing patterns and using non-linear dynamic models. Transformational organizational change will respond to the perceived uncertainties and dynamic capabilities being developed by firms.

The Corridor context, as described in detail in Chapter 2, involved massive uncertainty and high levels of turbulence so Level 1 above was certainly not the case facing Corridor participants with Level 3 highly likely and Level 4 in extreme cases with Level 2 being the case for several Corridor participants. This appreciation of uncertainty and required response influenced the strategy adopted in individual cases and by individual players. The case studies chosen help to how illustrate this occurred (see Section 8.2 for analysis and Figure 8.1 as an illustration).

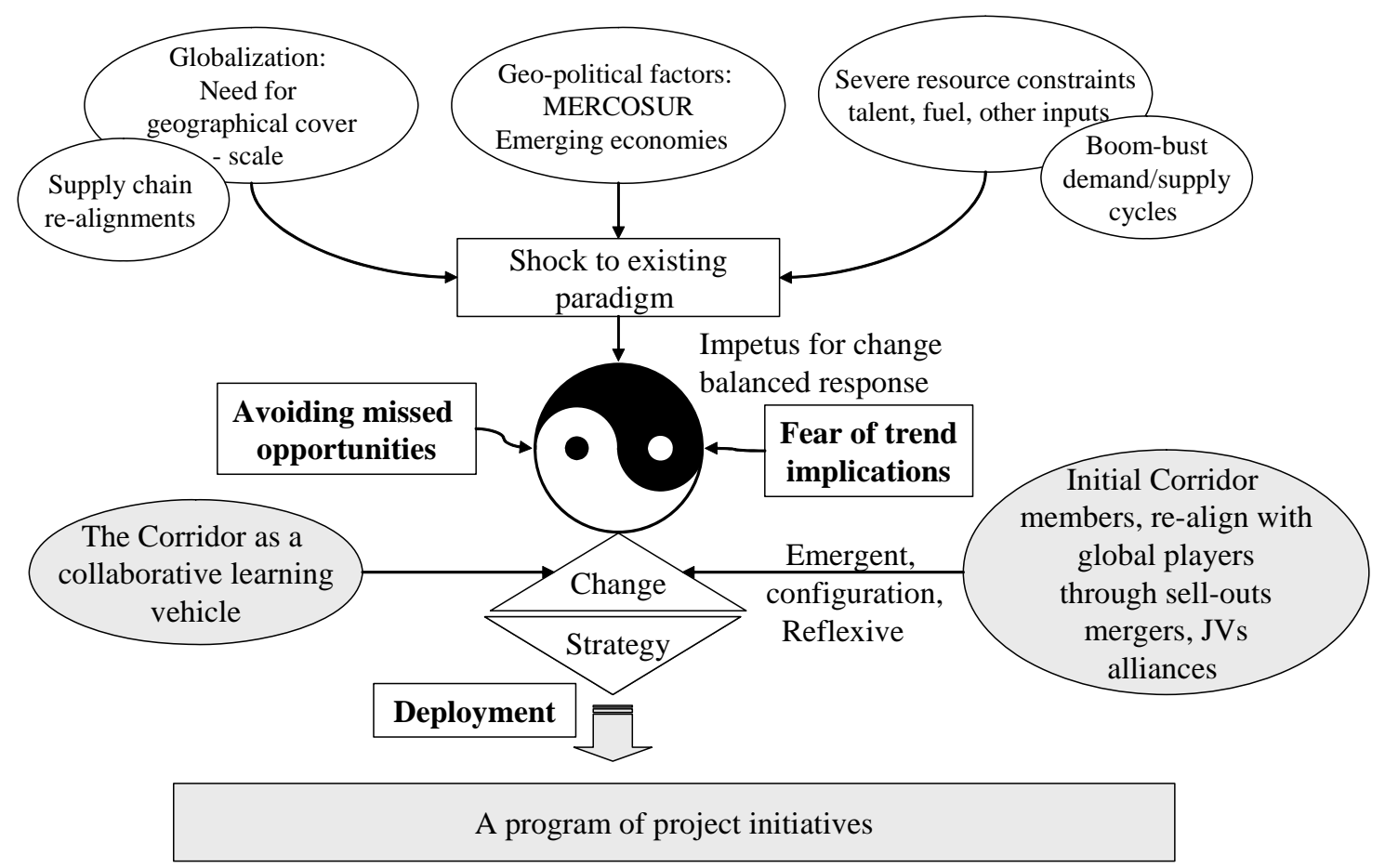

Figure 3.1: Understanding Corridor Development 
Figure 3.1 helps explain how the corridor evolved. This chapter will discuss the nature of globalization, geo-political factor and the way that the emerging economy has placed pressure upon the prevailing business paradigm in the MERCOSUR. This provided the kind shock often that triggers change and innovation (Kotter, 1995). This shock provided the impetus for change that Kotter (1995) refers to. The reaction can be explained by what Schein (1993) refers to as two types of anxiety. Anxiety 1 is the fear associated with an inability or unwillingness to learn something new because it appears too difficult or disruptive while anxiety 2 is the fear, shame or guilt associated with not learning something new - generally due to an explicit statement about the need for survival to learn the new things. Corridor participants related to type 1 anxiety as the fear of not being able to cope with the implications of the perceived emerging trend of globalization and the other drivers indicated as priming the 'shock' treatment feelings. Anxiety 2 was for them about the fear of missing opportunities that forming alliances and cooperative initiatives may present. Original Corridor members engage with global players in a number of ways that leads to collaboration and cross-business learning as well as joint venturing, alliances and even mergers and acquisitions. Both Regional and global players influence each other through the Corridor experience.

This chapter outlines the strategic response that became the Corridor initiative. The strategy was (in part at least) what Mintzberg, Ahlstrand and Lampel (1998) call emergent and configuration strategy. This provided a reflexive strategy in coping with the evolving situation facing them. Mintzberg et al (1998) describe and discuss 10 schools of strategy that fall into three groupsprescriptive, descriptive and configurative.

The prescriptive set of three strategic schools (the design school, the planning school and positioning school) is more concerned with how strategy should take place than with how it actually does take place. This view is very much about a deliberate top down, somewhat command and control, approach. This was popular in the 1980s and 1990s where those supporting these strategy schools believed that they could, and should, shape their preferred future. In part we see some evidence of this with corridor participants but generally strategy is used in the sense of designing the Corridor concept as a strategic response to the forces illustrated in Figure 3.1. The descriptive school sees strategy as an analytical process. 
The next descriptive set of six strategic schools is more concerned with describing how strategy does in fact take place and this help us understand how the Corridor functions with a diverse group of participants that individually often adopt varying strategic approaches. The Entrepreneurial School responds to market chaos and extreme turbulence and it focuses exclusively and extremely on a top-management perspective. This school sees strategy as being centred upon vision through the innate intuition, judgement, wisdom, experience and insight of the entrepreneur who formulates the strategy to meet the envisioned challenge. The fundamental basis of this school is rooted in innovation. The cognitive strategy school sees strategy as a mental process and take an interest in the way that a strategist's mind works by using cognitive psychology to do this - how they make decisions, how they judge evidence and how they communicate what they believe to be sound strategic decisions.

The learning school believe that strategy is an emergent process. This view of strategy presents a departure from the CEO (or highest echelon of management) being the strategy formulator, as is the view with the prescriptive strategy school, rather it implies a flow of influence stemming from across the organization from top to bottom. Strategy and changes emerge from the organization as a whole through its evolved culture and how the interplay and actions between leaders and followers affects procedures and governance. This chapter will later focus on more of this aspect.

The power school sees strategy as a process of negotiation linked to influence and politics. There was much evidence of power and its deployment being a key shaper of the Corridor concept as well as explaining observed behaviors. The politics school also believes that ability to influence and negotiate is also reflected by the sense of commitment or compliance experienced by those taking part in negotiations.

A related school to this is the cultural school that sees strategy as a collective process. Culture can be studied from either the outside in, or inside out. It can also be understood from the national/regional as well as organizational point of view to help explain what is observed. The environmental school sees strategy as a reactive process. This school sees the environment as the 
defining actor, together with leadership and the organization, shaping strategy through the organization's reaction to forces external to it. Mintzberg et al. $(1998, \mathrm{p} 288)$ argue that this school evolved out of contingency theory with ideas such as 'the more stable the environment, the more formalized the organization's internal structure'.

This last strategic group, comprising the configuration strategic school, is concerned with strategy as a process of configuration and transformation. Mintzberg et al. (1998, p305-306) states that Configuration School proponents believe that most of the time an organization can be described in terms of a kind of stable configuration of its characteristics for a distinguishable period of time adopting a particular structured by adopting its strategies to meet the challenges it faces from its external environment.

There are periods of stability and periods of extreme turbulence when disruptive change requires a quantum leap to another organizational form. These successive states of configuration and transformation occur over time similar to the stages of organizational transformation triggered by turbulent shocks described by Greiner in (Greiner and Schein, 1988; Greiner, 1998).

The key to strategic management therefore is to sustain stability most of the time when that maintains competitive advantage and to be able to shift gear to being transformational to CoPe with disruptive change to position the organization ready for the next fairly stable period. This is where the other strategy schools have their usefulness because the process of strategy making can be seen as Mintzberg et al. (1998, p305-306) argue as “one of conceptual designing or formal planning, systematic analyzing or leadership visioning, cooperative learning or competitive politicking, focusing on individual cognition, collective socialization, or simple response to the environmental forces: but each must be found at its own time and in its own context" - "the resulting strategies take the form of plans or patterns, positions or perspectives, or else ploys, but again for its own time and matched to its own situation”. Given the predominance of turbulence and uncertainty, and the need to step in and try to shape the future from this discussion of Courtney et al, (1997), an emergent strategy combined with a strong focus on the configuration strategic school 
approach was generally adopted to help shape the future and respond to events illustrated in Figure 3.1.

Understanding the various strategic schools of thought is useful for helping us understand how the Corridor concept evolved and the behaviors of those that participated in it in the program of many project initiatives. The dominant and prevailing view of the dynamics of competition is that any industry, whether it is domestic or international or produces a product or a service, the rules of competition are embodied in five competitive forces: the entry of new competitors, the threat of substitutes, the bargaining power of buyers, the bargaining power of suppliers, and the rivalry among existing competitors (Porter 1998, pp4-11). Porter argues that the collective strength of these five competitive forces determines the ability of firms in an industry to earn, on average, rates of return on investment in excess of the cost of capital plus the value of the managerial input. Naturally, the strength of the five forces may vary from industry to industry and may evolve as a given industry changes.

Put in logistic terms and referring this to the MERCOSUR region, each of the five forces may be clearly represented in the following manner:

1. Entry of new competitors: global shipping companies; global port operators; worldscale freight forwarding companies and logistics operators; global air cargo carriers; global coastal feeding and barging operators; extra-regional railway and trucking conglomerates;

2. Threat of substitutes: innovative distribution channels; new technology making all previous approaches obsolete; new type of containers; new stowage or transfer technology; better pricing or faster timing in ground transportation vis-à-vis barging operations; efficient railing vis-à-vis congested air stations; global accounts with extraregional headquarters; concentration of shipbrokers beyond the region;

3. Bargaining power of buyers: large global multinationals on the export or import process representing enormous volumes in the container trade; large brokers or nonvessel operator common carriers ${ }^{7}$ gathering great volumes of cargo; mineral and grain

\footnotetext{
${ }^{7}$ NVOCC: non-vessel operating common carrier are normally independent logistic operators gathering a number of shipper and exerting pressure on the carriers to obtain lower freight rates. Their bargaining power may naturally vary according to what their volume may be at a given point as well as the intended time-period.
} 
global dealers taking advantage from a raising cycle in the value of their commodities and exerting great pressure on services; global ocean carriers holding large economies of scale and better port pricing; shippers associations;

4. Bargaining power of suppliers: large oil companies exerting pressure on a strategic input for almost all the transportation chain; large international port terminal consortia concentrating global interests; large steel manufacturing firms choosing priorities between supplying oil and gas customers or the ship-building industry on regional basis; limited number of barges leading to a general rate increase on the waterways and costal system; and

5. Rivalry among existing competitors: national, regional, and global ocean shipping companies struggling for a market share by reengineering their services and organizational structures; ocean and river container terminals striving to prevail and control trades by lowering fares and upgrading technology; port hinterland competition among regional ports by creating rail access or expanding into distributing-related services; trucking, railway, or barging operations struggling for controlling a market segment ; global air-cargo carriers and regionally-based companies; provinces and departments disputing the control and operation of strategic corridors linking consumption and production poles, regions and countries.

The five forces may determine industry profitability because they influence the prices, costs, and required investment for a firm to remain competitive. Large exporting or importing multinationals may influence the freight rate levels an ocean shipping company may charge, as does the threat of substitution. Similarly, buyers' power may have an impact on the level of investment a given carrier must deploy in order to match their customer expectations on service level, quality, and delivery times.

The bargaining power of suppliers may become critical for most transportation operators, especially during times where the oil market shows instability and uncertainty for an input of a high strategic value such as fuel oil. The intensity of rivalry has a direct connection with the available supply or the number of companies present in a given market, and with the freight level logistic companies may charge their customers at a given moment. These factors are crucial 
elements for the Corridor. As well as the threat of entry they place a rather clear limit to freight rate levels and extensively shapes the degree of investment needed on technology, structure, and management to deter entrants. A commonly held view about industry profitability is that profits are a function of the balance between supply and demand, where the profitability emerges as a consequence of the demand being greater than the supply.

Although true and as argued by Porter (1998, pp8-11), this focuses only on short-term fluctuations in supply and demand, whereas long-term profit projections are somehow dictated by the industry structure. Industry structure may determine how quickly competitors add supply as well as it may trigger a price war and reduce profitability every time there is an excess of capacity - a phenomena that take place too often in the container trade for instance. However, this varies from industry to industry.

Durell (2005) argues that in the shipping industry for example, the industry structure plays a key role as regards to what is known as the shipping cycle. When the balance of supply and demand is more or less in equilibrium, ocean freight rates are reasonably stable. In this state, the main incentive to build new ships is to replace aging tonnage with newer vessels that are more economic and efficient to operate. With an average vessel life of 20 years, about $5 \%$ of the world's fleet is either scrapped each year or sent off to what is know the laid-up market - some remote sheltered locations that have safe anchoring in the Norwegian fiords or in Greek waters.

If the global demand for shipping starts to exceed its long-term average growth rate, then tonnage capacity becomes constrained. The first thing that happens is that the scrap rate drops to about 1\%. Then if demand really ramps up, as happened in 2003 and 2004, supply just can't keep up. New ships must therefore be ordered, and that creates backlogs for shipbuilders, which will generally require a couple of years to catch up with demand, creating logical market disruptions and impacting on their entire value chain. Problems then begin to surface as ship-owners seem to mimic the actions of momentum investors ${ }^{8}$ piling into the latest hot stock as their new-building frenzy leads to an oversupply of expensive new shipping tonnage in the market.

\footnotetext{
${ }^{8}$ These are stock market investors who follow a herd instinct by piling their money into the latest 'hot' stock
} 
The problems of oversupply are exacerbated by governments giving massive grants to shipbuilders to secure jobs and tax breaks to ship-owners, such as 100\% depreciation in the first year of operations as well as operational subsidies as is the case for the American merchant marine ${ }^{9}$. In a normal cycle, ocean freight rates plummet, not only because of the oversupply of tonnage but also because demand eventually slackens and this leads us into the "bust" part of the classic boom-bust cycle.

An explanation for the apparent indefinite continuation of a "boom" in shipping is that the enormous growth in demand from China and India for energy and raw materials will be sustained for many years if not decades, thus sustaining the demand for ships through flooding the world markets with products which eventually triggers demand for bigger vessels capable of reaching distant markets. Such a cycle brings about (in response to an extraordinary excess of demand arising out of China and India's constant growth) a sort of markets interdependency. This cycle has evolved to such a degree that even the far away South American (east and west) coasts have witnessed the arrival of global shipping companies that saw an opportunity to expand their services beyond the east-west-east traditional shipping routes.

The "laid-up market”, where older and lesser economic and efficient ships are anchored, is often slow to react before a market boom situation because vessels have to be put into seaworthy conditions first, minimally crewed, and repositioned in the trades of interests. This is similar to other large capital provision industries such as the building development industry where developing new-buildings takes some time to realize and often trigger a real estate oversupply impact on the market. Along with the shipping expansion, global port operators also saw an opportunity to accompany this expansion and also explore distant markets. In fact most logistic segments of the supply chain attempted to gain global reach in the same way. The industry structure seems therefore, to play an important role in the interaction of Porter's five competitive forces and especially when those industries are of global nature or global reach (e.g. ocean shipping; ports; ship-building).

\footnotetext{
${ }^{9}$ The American merchant marine runs an annual program of subsidies aimed at helping its merchant marine remain competitive on its operations around the world, and foster shipowners place their construction orders on American shipyards.
} 
Probably the three most widely cited books on competitive analysis from the 1980s were Michael Porter’s “Competitive Strategy” (1980), “Competitive Advantage” (1985), and “Competitive Advantage of Nations” (1989). According to Porter, strategies allow organizations to gain competitive advantage from three different bases: cost leadership, differentiation, and focus. Porter calls these bases generic strategies. Cost leadership emphasizes producing standardized products at a very low per-unit cost for consumers or segments that are price-sensitive.

Differentiation is a strategy aimed at producing products and services considered unique industry-wise and directed at consumers who are relatively price-insensitive. Focus means producing products and services that fulfill the needs of small groups of consumers. Porter's strategies imply different organizational arrangements, control procedures, and incentive systems. Larger firms with greater access to resources typically compete on a cost leadership and/or differentiation basis, whereas smaller firms often compete on a focus basis. This is where the issue of achieving larger economies of scale within the Corridor proves to be of paramount importance for all those companies concerned about being relegated to perform secondary services as a minor industry player or being outsourced by the global and more resourceful firms. Achieving project management efficiencies and developing skills and competencies to compete with global players becomes essential in order to honor the often urgent deadlines set by a volatile business environment. It also became a strategic reality for all these companies, who needed to compete on a cost leadership basis, to start searching for potential allies interested in entering in some sort of alliance, partnership, or joint-venture.

Porter (1985) stresses the need to achieve larger economies of scale, accomplish a higher level in technology, having preferential access to raw materials - among other factors - to carry out a successful cost leadership strategy. In this regard it is important to point out that most if not all of the players within South America (no matter what type of logistic segment in the supply chain) had to face the arrival of global players showing strong cost leadership strategy attributes. As such, the newcomers' economies of scale turned out to be substantially bigger. Consequently their bargaining power was much stronger for gaining access to strategic raw material and resources (e.g. fuel oil ; building and operation subsidies). This resulted in these global players 
being more accessible in the region, therefore far more efficient, and their technology also made a distinguishing difference due to the daily running costs of their more modern ships, better equipped terminals, faster transfer equipment, etc.

Porter (1985) also highlights that a firm that can achieve and sustain differentiation will be an above-average performer in its industry if its price premium exceeds the extra costs incurred in being unique. As such, the differentiation strategy logic lies in the attributes chosen by a firm to differentiate it from its rivals. Naturally, such a choice entails being able to afford the required additional investment. For those (by then totally isolated) companies operating in South America this investment could be seen as unrealistic. They stood on the verge of facing truly global, aggressive, and resourceful firms capable of successfully adopting both a cost leadership and differentiation strategy in their intent to capture most of the main existing market segments while outsourcing those of minor potential.

The last generic strategy is focus, the choice of a narrow competitive scope within an industry. This presented a potential and threatening scenario that served as a triggering element to constitute the Atlantic Corridor of the MERCOSUR for their members to expose their views, combine their resources, enter into restructuring alliances, leading all to face the new era of market globalization in the logistic industry.

Finally, Porter (1985) also refers to a last option defined as "stuck in the middle" or the manifestation of a firm's unwillingness to make choices about how to compete. Such a scheme turns out nowadays simply unthinkable since a firm that chooses to adopt this strategy, would compete at a disadvantage because the cost leaders, differentiators, or focusers will be better positioned to compete in any segment.

However and surprisingly, this has been the case for various large regional shipping companies and port terminals operating under the flag protection scheme ${ }^{10}$. Restrictive laws had let them grow large and remain "stuck in the middle”, facing no threats, and within a market environment

\footnotetext{
${ }^{10}$ Flag protection schemes were implemented by most countries in South America during the 70s and 80s to enforce a share of their imports and exports be carried by vessels flying their national flags.
} 
that lacked of competition and discourage the utilization of any of Porter's generic strategies for a long period of time (e.g. Lloyd Brasileiro; Empresa de Lineas Maritimas del Estado - ELMA; Flota Fluvial del Estado; Libra Navegacao) ${ }^{11}$. Interestingly, the same concept of market protection leading to a stuck in the middle corporate attitude is still established in developed countries such as Canada or the USA, where the lack of competition brings about the utilization of older tonnage and obsolete technology - contrary to on-going world trends (e.g. coastal trade in the USA; barge trade within Canada's Great Lakes).

Naturally and with the advent of both the globalization process and the creation of the MERCOSUR market, flag protection schemes and bilateral treaties drew to a close and new market rules emerged to make room for new players to enter and lead the way to both market and industry changes. These new trends impacting on regional shipping and port business is well developed and explained in a policy research project report undertaken by the Lyndon Johnson School of Public Affairs of the University of Texas at Austin (Boske, pp35-60).

Thus the Porter forces model that he still felt applicable and appropriate for the e-economy as for example (Porter, 2001), can be used to help explain the shipping cycle as it occurs in the Latin American context, which is the context of this dissertation. Further, the formation of alliances and pseudo and real joint ventures has been offered by a range of strategy thinkers and academics such as (Doz and Hamel, 1998) as a strategic response.

This entails cooperating with competitors, co-opting them and learning how to internalize knowledge from them. Doz and Hamel (1998) document cases study examples from a range of industries. Walker and Hampson (2003) show how building construction organizations can form strategic alliances. Oum, Park and Zhang (2000) provide extensive examples from the air transport industry and Ferdows (1997) illustrated how foreign factories can be strategically linked in a value chain that fits very well with the way that Corridor participants arranged themselves in their value chain.

\footnotetext{
11 State-owned and private shipping companies in Brazil and Argentina that got bankrupt or sold out.
} 
All these examples support the earlier part of this section that the resource based view of the firm helps explain behaviors such as those that led to the development of the Corridor. We see that these alliance-type relationships not only allow more effective sharing of risk through superior application of scale and depth of resources but also application and transfer of knowledge to help dynamic competencies develop and be engaged so that these Corridor organizations have better reflexivity and adaptation capacity to rapidly changing global competition forces. Many of these aspects will shortly be discussed in more depth.

\section{3 - COMPETITIVE ADVANTAGE AND THE VALUE CHAIN}

This thesis' context resides in a transformation of logistics companies that sought competitive advantage. Logistics and supply chain management (SCM) is generally linked in the mind of practitioners and academics (Sadler, 2007). Further, following the research direction 3 of Winter et al. (2006) in which value is privileged over focus on techniques and tools, SCM as a technique is re-framed in terms of value chain management. This view is supported by the leading literature for example Sherer (2005, pp77) states that “A broader concept, value network advocacy, better describes the needs of business today.” Later she states "Today we should be focusing on networks, not linear chains or flows. This applies to the physical process and even more so, the movement and sharing of information. Information has become the key driver of advantage. Information hubs should be the basis of communication Information technology today can support this move.” (Sherer, 2005, pp81). This provides one of but many examples from the literature on how SCM has been evolving towards a value focus as is the focus of this thesis. Readers wishing to find a current literature review and extensive discussion on the trends should refer to Sadler (2007).

It is useful to apply Porter's model of the value chain to better understand the process through which the various regional logistic segments proceed when facing new global trends. Porter (1985, pp36-61) argues that the value chain is made up of value activities (the distinct activities a firm performs) and margin (the difference between total value and total cost arising out of performing the value activities). 
Value activities can be classified in to two broad types, primary and support activities. Typically, primary activities are defined by the creation of a product or service (inbound logistics / operations / outbound logistics / marketing and sales / service), whereas support activities (human resources / technology / procurement etc) functionally support the primary activities. This leads to the creation of margin.

An important aspect that forms part of the support activities is the firm's infrastructure which is precisely one of the issues that renders value to the Corridor. The infrastructure created through the Corridor is useful in providing and making available tools for companies with smaller relative infrastructure resources. Those regional companies facing difficulties in tackling new global market trends and experiencing their respective infrastructure levels as a burden rather than as an advantage, found in the Corridor roundtables both competitors and complementary firms experiencing the same problems.

The Corridor gave them the opportunity to search for new ideas, new resources, and new concepts which in general contributed to enrich and strengthen their own value chains in a wide array of forms. These forms often entailed restructuring and adapting companies to face new scenarios by implementing project management processes and techniques. These new organizational forms manifested themselves as strategic alliances, joint-ventures, and different degrees of partnerships. It even brought about a number of mergers and acquisitions among members, as a way to enlarge economies of scale for them to remain competitive with larger global infrastructure resources.

Both primary and support activities of the participating members tended to improve by exposing what their limitations and problems were, and (most of the times), finding alternative action to take by combining efforts and gaining one another's experiences.

For the purpose of letting the reader better comprehend the extent of the role played by each of the primary and support activities among the Corridor participants, it is useful to follow Porter's value chain classification as a way to clearly conceptualize the "why”, "what", and "how" of the Corridor along with the roundtables. 
Porter refers to five generic categories associated with the primary activities:

1. Inbound logistics: all those functions connected with receiving, storing, and disseminating inputs to the product, such as material handling, warehousing, inventory control, vehicle scheduling, and returns to suppliers. However for companies involved in rendering logistic services, there is no product to be manufactured, but that just a pure service is to be delivered. This presents a different perspective. For an ocean shipping company, inbound logistics is represented by the handling of their entire fleet of containers spread all over the world. This involves activities associated with trucking operations, customs operations, warehousing and distribution operations, stuffing and un-stuffing ${ }^{12}$ of containers allowing a good turn-around-time (TAT) of the container itself $^{13}$, and naturally the positioning and repositioning concept which comes associated with the concept of balance costs. Having a low TAT along with a large fleet of containers disseminated over a rather large geography, is considered within the industry as a very dangerous situation - both operationally and financially. An efficient bunkering operation at strategic world locations is also regarded as a key value chain variable, with fuel oil being the most expensive input for running a shipping fleet. Smooth handling of spare parts and accessories reaching navigating convoys at remote locations along with an efficient bunkering operation at the right time and location, turn out simply essential for a waterways operator. For an air-cargo company, bunkering is also of paramount importance, as well as reliance on efficient ramp services for a smooth operation. For a port terminal, inbound logistics is very much related to its infrastructure in terms of transfer facilities, cold-storage supplies, fluids and tools for maintenance, spare parts logistics, automation and satellite communication equipment. Further examples can be presented but what is important at this point is to highlight the distinction for a logistic company between Porter's first generic category of the primary activities and the operation itself, and to understand the role that economies of scale may play in this context. Global firms relying on global purchasing power and global distribution will easily be differentiated with those

\footnotetext{
${ }^{12}$ In shipping the term 'stuffing' is used for filling literally with stuff of all kinds

${ }^{13}$ Time a container takes to complete a round-trip from port to door delivery and back to port.
} 
nationally or regionally-oriented companies showing much less versatility and maneuvering capacity. This illustrates the need for firms to be reflexive towards the context in which they operate;

2. Operations: capital concentration here is a destabilizing factor for regional shipping companies. This is because economies of scale displayed by the global carriers are far larger than those achievable at regional level. According to the Economic Commission for Latin America and the Caribbean (Hoffmann 1988), concentration itself has affected everything including ship size, the number of port calls, and changes in shipping services offered. Haralambides (2000) stated at a conference that “The overall degree of containerization, currently about $60 \%$ of general-cargo traffic, is expected to reach $70 \%$ or higher by the year 2010. Total container traffic now exceeds 200 million TEUs (twenty equivalent units) per year”. He also stated that between 1991 and 2000, container shipping capacity increased at an average annual rate of $10.5 \%$, compared to 2.5\% in worldwide GDP, $6.5 \%$ in international trade, and 2.1\% in world merchant fleet. This suggests that an expansion of the global carriers toward achieving larger economies of scale reinforced by a globalization of world trade is lowering the entry barriers for global carriers to many world markets. Therefore, the Corridor concept appears to be a strategic project arising out of a defensive reaction to the expansionist process of global corporations. Whether this results in more competitive operations or not is a subject to be dealt with in the coming chapters;

3. Outbound logistics: this is normally associated with collecting, storing, and physically distributing the product to buyers - a typical logistic function. This is the very nature of a logistic operator's business comprising: distributing products to the end buyers, conducting efficient warehousing operations, and designing a precise scheduling for air-traffic or marine-traffic services, etc. ECLAC ${ }^{14}$ argues that changes in shipping services have come about as shippers have begun to expect higher quality services, including shorter transit times, more direct services, guaranteed delivery times, doorto-door ${ }^{15}$ services, low incidence of damage, and the use of electronic communications

\footnotetext{
${ }^{14}$ Economic Commission for Latin America and the Caribbean (ECLAC). Concentration in Liner Shipping :its causes and impacts for ports and shipping services in developing regions by Jan Hoffmann (Santiago, Chile; August $17^{\text {th }}$ 1998). P32

${ }^{15}$ Definition given to a container service going from the supplier's premises up to the end receiver's plant.
} 
and systems that have received ISO 9000 certification. Naturally, outbound logistics again has a direct link with infrastructure and therefore with economies of scale. Larger and more resourceful firms are in a better position to achieve larger economies of scale that lowers their base-cost, and through this, attain competitive advantage;

4. Marketing and Sales: activities such as pricing, channel selection, channel relations, advertising, promotions, and sales force deployment are of strategic importance to induce a customer to favor a given service at the expense of a less resourceful one. Typically, global companies that gained larger economies of scale based on the eastwest-east trades (eastbound and westbound services), found themselves in a much better position to expand their services to cover the north-south-north trades (northbound and southbound services) to national or regional companies going global. Global companies do this by making use of their already existing marketing and sales services. ECLAC argues that globalization has affected both trade direction and volume, blurring the distinction between north-south-north and east-west-trades. Reliance on transshipment means that a portion of the north-south trade is transported by east-west carriers for part of the journey. In addition, seasonal cycles, trade imbalances, and route differences add to the difficulty in defining trade directions; and

5. Service: activities associated with providing service to enhance or maintain the value of the product, such as installation, repair, training, parts supply, and service adjustment. Again at this point, relying on larger economies of scale allowed the operator to become more competitive based on lower running costs and repositioning costs worldwide for an entire fleet - either for ships, aircraft carriers, trucks, or railways.

Porter (1985, pp40-45) also refers to support activities. These played a fundamental role for the MERCOSUR operating companies at the time of facing the arrival of those global players who held greater economies of scale, as well as an immediate apparent greater capacity to achieve a differentiated competitive advantage form in procurement; technology development; human resources; and firm's infrastructure. 
Being global and having access to the key locations for acquiring fuel oil (FO) ${ }^{16}$, or having a world coverage for rapid deployment of flying in spare parts, as well as obtaining both available space and competitive pricing for ships' dry-docking ${ }^{17}$ on regular basis, are variables that define a differentiation competitive advantage for the global and more powerful firms, at the expense of those showing lesser volumes and number of assets to negotiate with. In the case of truly international industries such as ocean and air transportation, global mobilization of assets to the key supplying locations delivers a strategic difference and helps improve profit margin within the value chain model.

Technology development can be represented by a number of factors. These include the higherrelative speed of vessels, a faster loading/unloading capacity of gantry cranes ${ }^{18}$, a well developed on-line cargo tracking on the part of the NVOCCs ${ }^{19}$, GPS-based satellite system to track down containers over a certain hinterland, etc. The combination of technology and a large client-base of global reach, provides a differentiation competitive advantage that is very difficult to offset for a national or regional firm whose market boundaries were so far well defined and not subject to expand and therefore forced somehow to innovate.

Human resource management affects competitive advantage in any type of logistic company, through its role in determining the skills and motivation of employees and the cost of hiring and training. Large and global shipping companies recruited their future executives from within the MERCOSUR region and sent them over to their corporate universities located across the USA, Europe, and Asia, not only for professional training purposes but also to be indoctrinated in the firms' corporate culture and to become familiar with the company's operating systems and ways that 'things are done'. This is directly connected with knowledge management (KM) and where

\footnotetext{
${ }^{15}$ Fuel oil can be supplied in different parts of the world at different prices for which it turns out crucial for the ocean carriers to access to those spots first, and count on enough volume to obtain price differentials enabling them gain competitive advantage on the north-south routes.

${ }^{17}$ Vessels normally go dry dock once a year for a period of 30 days for operational maintenance, further to a compulsory inspection every 4 years.

${ }^{18}$ Specialized port cranes utilized to load and discharge full-container ships worldwide.

${ }^{19}$ Non-Vessel Operating Common Carriers: definition utilized within the USA for those companies that count on a large number of clients whose cargoes get shipped worldwide on third party vessels.
} 
perhaps the Corridor's participants knew how to better respond to the globalization trends. This will be dealt with later, both in this chapter (see Section 3.7) and throughout the dissertation.

Finally, the firm's infrastructure turns out to be of paramount importance when assessing the real threat that globalization has posed to the regional Corridor firms. The threat to Corridor participants lay in the capacity of global companies to deploy a tremendous amount of assets and resources of all kinds including:

- number of trades operated by a single shipping firm;

- global sales and operational offices;

- number of trucks and trailers operated by a small number of ocean carriers;

- integration between railways and shipping lines;

- expansion of global port terminals and deployment of innovative and capital-intensive transfer equipment ${ }^{20}$; and

- highly specialized ships deploying state-of-the art navigation and operation technology.

It becomes apparent that both the primary and support activities played a fundamental role in reshaping the region's value chain - as defined by Porter - in that every single operator within the MERCOSUR has witnessed changes in its business environment in a quiet but dramatic fashion. In that process, many companies have vanished whereas others have been sold out to the foreign and global operators. However, there were a significant number of firms that found in the Corridor an opportunity to face a threatening scenario. Porter's theory helps in analyzing the way that forces act and interact and they make value chain analysis, along with implications that may be experienced so that discrete threats and opportunities can be better identified by the value chain segments.

This section drew to particular attention in context-specific terms, how viewing logistics from a value chain as opposed to simply a supply chain, has relevance to this thesis. As stated in Section 1.8 with reference to the Winter et al. (2006) research direction 3 , research is needed into how value is created and delivered in projects

\footnotetext{
${ }^{20}$ Rubber-typed cranes, fork-lifts, straddle carriers and further handling equipment that is utilized to transfer cargoes from port sheds to trucks.
} 


\section{4 - THE THEORY OF THE FIRM AS IT APPLIES TO THE MERCOSUR REGION}

The nature of the firm can be viewed from a number of perspectives. The resource based view (RBV) sees organizations as a set of competencies and resources providing competitive advantage that can be successfully used (Conner and Prahalad, 1996). Competencies have been argued as being a vital resource (Prahalad and Hamel, 1990) including knowledge (Conner and Prahalad, 1996). Organizations, such as those in the Corridor, needed to respond to turbulence and market uncertainty through developing a set of dynamic capabilities that allow them to be flexible, lean, responsive to disruptive change and become learning organizations so that they can interact more effectively with both upstream and downstream supply chain members (Spender, 1996; Teece, Pisano and Shuen, 1997; Eisenhardt and Brown, 1999; Eisenhardt and Martin, 2000).

The theory of the firm aims at assisting in the theoretical investigation of one of the central problems of economic analysis - the way in which prices and the allocation of resources among different uses are determined. These probably are the main triggering variables to constitute the Corridor project. The conditions of equilibrium analysis require that there be some force that prevents the indefinite expansion of output of any firm. If a situation of pure competition is assumed, the limit to output is found only in the assumption that the unit cost of a product or service must rise at some point when even additional quantities of it are produced or services are rendered —putting more resources in lower unit cost ceases to generate additional revenue to cover that investment and indeed revenues may actually decrease with additional input of resources to effect efficiencies that are aimed at lowering unit costs.

Typically, this theoretical limit seems to be evident when analyzing the case of all those global ocean carriers that commit themselves to building larger and larger vessels in pursuit of achieving greater economies of scales to reduce their slot $\operatorname{cost}^{21}$. Many if not all of them are probably well aware that when shipping supply exceeds demand only a few ports worldwide can be relied upon to accommodate the needed facilities to provide a berth for these ships that seem to be built larger

\footnotetext{
${ }^{21}$ The cost assigned to carry one TEU or twenty equivalent unit on board a ship. e.g. a 20 footer container on board a ship.
} 
and larger as the pressures for globalization intensify. However, Penrose (1995, pp13) argues that "When appropriate resources are available, a firm can produce anything for which a demand can be found or created, and it becomes a matter of taste and convenience whether one speaks of the market or of the resources of the firm itself as the consideration limiting its expansion".

It appears that perhaps the global shipping firms believe that they can create their own niche demand and dominate the logistics industry by using their economies of scale for cost advantage and their sophisticated skills for differentiation and position competitive advantage that is provided by their application of vast resources available to them. These global companies have a good understanding of the time lag between a market signaling the enormous recent increased demand for shipping services and constrains and barriers that inhibit rapid responses to these signals by all players.

The MERCOSUR market has witnessed global logistic and transportation firms engaged in a race:

- to build larger and larger ships in an intend to achieve larger economies of scale scales and lower per-unit costs;

- for firms acquiring national and regional assets to become global players or simply enlarge their scope of operations, and gain wider and more extensive market coverage (e.g. railways in Argentina and Brazil ; port terminals all over South America ; huge distribution hubs spread all over the region); and

- foreign firms acquiring assets on the waterways to intend playing a dominant role in the rivers linking five countries in the region.

All this contradicts the firm's limits to growth theory, but that seems to find a certain logic with Penrose's concept of what firms usually do. Cyert \& March (2006, p179) argue that prices and outputs change primarily in response to short-run feedback on performance and the extent to which solutions to organizational problems are sought in price and output changes, and that this depends on past learning about the consequences of that changes. They further contend that firms do not resolve potential conflict between market share, profits, and output stability goals 
primarily by a procedure of explicit mediation. Rather, they ameliorate conflict by accumulating excess resources, by decentralizing information, and by attending sequentially to crises.

It has been the author's experience after attending a number of roundtable sessions and witnessing the globalization wave approach the MERCOSUR coast, that the way the Corridor members regarded the global operators did not consciously reflect upon and appraise prices, their past experiences, their past learning, or any relationship between organizational structures and price/output variables. On the contrary, the apparent unstoppable race of the global companies for becoming even more global by absorbing distant markets that they had been absent from for so long, seems to have no direct connection with any theory of the firm's limit to growth or price and output determination per se.

This suggests that the globalization process is first of all, interpreted and then put into practice as an expansionist strategy by a number of large-scaled leading global firms that intend to exert dominance upon their followers. This interpretation of the global threat that loomed large was likewise understood by the regional operators who eventually gave birth to the Corridor as a defensive reaction by a number of organizations.

Put perhaps in simple terms, the risk of not creating or generating global reach for those everexpanding international firms seemed to indicate firms being more relevant than the increasing costs that the strategy to form part of the Corridor might imply. Not being part of this Corridor entity could mean leaving room for others to emerge as Corridor members and gain dominance and an opportunity for Corridor members to be better positioned in the long-run to be ahead of the competitors.

Penrose further argues that contrary to a popular belief, large firms appear extremely successful when competing against smaller companies despite smaller firms having more simple administrative and operational structures. Furthermore, there appears to be little evidence that larger firms are managed inefficiently when there is enough time for them to make the adjustments and adaptations to their administrative framework appropriate to their increasing size. 
This is precisely what apparently happens, as explained earlier, with the shipping cycle in that the world maritime markets do no change overnight but that it takes months and even years to foresee a new trend developing. This new mother trend seems having been represented by the globalization process. What perhaps has not been well assessed on the part of the larger companies, is the uncontrollable long-run race for world market cover and the strategic positioning that may enable them hold a dominant position and diversify both horizontally in other logistic segments, as well as vertically along the supply chain.

Typically, there are a number of subsidies that are granted by some countries which tend to ease the expansionist process of some fleets in a sort of permanent pursuit of greater economies of scales and lower unit costs. These distortions tend to impact on the firm's practical application of lessons offered by free market and competitive advantage theories of the firm. An example of distorting influences that limit the application of pure theories of the firm is the Operating Differential Subsidies (ODS) and the Construction Differential Subsidies (CDS) granted by the American government aiming at maintaining a US-built and crewed merchant marine ${ }^{22}$. Another fundamental difference of perceptions and behaviors between global corporations and those of MERCOSUR-based firms with a lesser-relative market coverage that becomes apparent is suggested by their executives’ attitudes towards their firms’ expansion.

Penrose (1995) contends that the managers of global firms have much more to gain if profits can be retained and invested in the firm - contrary to the popular belief of making short run profits only at the expense of what the long-run might bring. Individuals thereby gain prestige, personal satisfaction in the successful growth of the firm they are connected with, more responsible and better paid positions, and wider scope for their ambitions and abilities. This does not mean that executives will limit their own salaries in order to leave more for profits. On the contrary, the salaries of top executives can get as high as the community will condone or as the conscience of the executives themselves will permit - stock purchase options and other bonuses being the main mechanism (O'Neill and Berry, 2002; O'Neill, 2006).

\footnotetext{
${ }^{22}$ See Regulation and Policies of American Shipping by MIT’s Ernst Frankel, Auburn House, Boston
} 
Buchanan (1940, p209) states that: "It has been argued, for example, that an enterprise attempts to maximize net income to its owners, but that this is equivalent to maximizing the present worth of its assets - for the significance of the assets to the firm is their ability to contribute to the realization of the desire stream of dividends to shareholders having the greatest present worth".

Both operational and building subsidies granted by many of the maritime nations whose companies are engaged in the global race for greater economies of scales and lower operational costs, act as a permanent driving force toward the logic of accomplishing the greatest present worth. Increasing present worth means gaining access to new markets and becoming more and more global, achieving greater economies of scales and consequently gaining more negotiating power, as well as displaying merits to qualify for geopolitical-oriented subsidies backing up the expansion strategy of many firms in the ocean shipping and port business.

On the other hand, small businessmen frequently tend to identify themselves with their firm and to view it as their life's work, as a constructive creation to which they can point with pride and which they can pass on in full measure to their children. To this end, it may be asserted that they often prefer to reinvest their profits in the firm rather than outside and to draw only moderately on profits for their personal consumption.

This assertion is supported by a study of over 500 small and medium sized businesses in New Zealand where eight factors of motivation for starting a business were identified. Tax reduction was only rated at 1.7 out of 5 , approval of others was rated 2.2 and following a role model was rated 2.6. Personal development was rated 3.8, instrumentality of wealth 4.2, family and community welfare and need for independence 4.3 and employment being rated more highly at 4.4. (Pinfold, 2001).

This study, though not intended to be representative of Corridor participants, nevertheless is indicative of the small family-type business sector and supports behaviors observed and sentiments expressed in many discussions with Atlantic Corridor participant senior managers. Business sustainability and growth appears to be a common goal for both large global companies as well as smaller regional operators. 
It seems reasonable, therefore, to assume that in general the financial and investment decisions of firms are controlled by a desire to increase total long-run profits. However, here again both economies of scale and competitive advantage are being of crucial relevance to dominate a region or market that was previously conceptualized or understood by its operators in a totally different manner. In many industries and areas within the Corridor scope of action, there were a considerable number of firms that have been operating successfully for several decades under competent and imaginative management, but have refrained from taking full advantage of opportunities for expansion.

Many of these were family firms whose owners were content with making a comfortable profit and have been unwilling and unmotivated to make more money or to raise capital through procedures that would have reduced their control over their firms. This process has also helped global companies gain a foothold into the region by acquiring such firms and aligning into their global operating scheme. Thus strengthening or driving even further the Corridor's very essence seems a useful mechanism for holding off total domination of the MERCOSUR by global companies at the expense of regional companies in a way that the local firms could not participate in and shape.

It is accepted that there are three types of explanations of why there may be a limit to the growth of the firm, regardless of whether it is a large, small, global, or regional firm. These are managerial ability, product or factor markets, and uncertainty and risk. The first refers to conditions within the firm, the second to conditions outside the firm, and the third is a combination of internal attitudes and external conditions. Regional management was to a great extent first captured by the global companies in their intent on recruiting regional talent and making their inducement periods shorter. ${ }^{23}$ Consequently, this process weakened many of the MERCOSUR-based firms whereas it tended to shape what was later regarded as a global threat to the region. Managerial recruitment was also connected with these companies’ ability to tackle

\footnotetext{
${ }^{23}$ Global shipping companies such as MAERSK, EVERGREEN, MSC, or P\&O, tended first to recruit local or regional talent to later formulate and implement their market enlargement strategies.
} 
ongoing levels of uncertainty. This later proved being less relevant when compared to these firms' need or most highly rated strategy to expand and go global before others did.

Regarding the external barriers to expansion, it is reasonable to assume that there is not an effective limit to the amount of any kind of productive resource that a firm can obtain at a given price, and that there are opportunities of profitable investment open at existing prices and interest rates - regardless of what the situation of a given economy may turn out to be. MERCOSUR's barriers of entry can be considered as low. This, together with the global firm's threat of looming large to gain economies of scales, provided the kind of shock illustrated in Figure 3.1 because these global firms turned what theory defined as possible limits to the growth of the firm, into their favor. These limits were in fact evident as the currently operating national and regional companies saw themselves gradually constrained with respect to:

1. keeping their managerial ability to expanding and gain economies of scales in view of their relatively smaller operated volumes and limited financial opportunities (a resources limitation); and

2. face a new phenomena that shaped a totally new business environment that none was prepared to predict and overcome (a strategic limitation).

While regional managers started giving up their regional positions to go global and pass their knowledge on to the newcomers, the latter brought the strength of their economies of scale and lower relative costs to set a remarkable competitive difference that would help them gain market share while either displacing existing players toward a secondary role of feeding operations ${ }^{24}$ or simply displacing them from the market - forcing them to sell, enter in some sort of alliance, or simply give up and disappear.

The theory of the firm argues that the expansion of firms is largely based on opportunities to use their existing productive resources more efficiently that they are being used - provided that there is not a monopoly condition but that of a free market. Firms become larger and larger, and the

\footnotetext{
${ }^{24}$ Feeding companies are those being outsourced to carry out operations of minor scales or volumes between coastal ports or non-distant geographical locations (e.g. 500 TEU vessels linking a hub port terminal and a non-regular port of call ; trucking companies being outsourced by major railways to render door delivery services as from the railhead ; outer port empty container terminals outsourced by main port terminal operators)
} 
question arises whether the large firms are more efficient than smaller firms would be because they are larger with more resources. This raises interesting questions. Does this justify the assumption that they will become more efficient as they grow? or that each of their segments of activity will become cheaper, of better quality, or more adapted to what exporters and importers want?

While growth is a process, size is a state - which means that the Corridor is either a result of, or a reaction to, both growth and size variables. Both can be regarded as a direct threat to the smaller regional firms’ very regional existence.

In this regard, Penrose (1995, p131) highlights the point that the profitability and even survival of a firm depends entirely on the ability of its entrepreneurs to make shrewd financial deals, to judge correctly market changes, and to move rapidly from one type of activity to another. Firms fail to be economically sustainable when they do not adequately concentrate on the intensive development of any of their existing segments of activity, but instead jump haphazardly from one type of service to another in response to often short term changes in external conditions. Put in logistic terms and emphasizing the purpose and goals of the Corridor, Hoffmann (1998, p12) highlights that there has been a substantial move toward stronger alliances and mergers - perhaps an inevitable stage the Corridor members had to face at a given point.

Unlike Conferences $^{25}$, a major goal of transport logistics alliance is to reduce the cost of transport. While keeping their individual corporate identities, alliances allow liner shipping companies to combine cargo volumes, increase service frequencies, and increase vessel use through vessel sharing agreements (VSA) ${ }^{26}$. Similarly, terminals, equipment, and containers are shared, and companies are able to use their collective financial strength in order to procure and replace long-term assets. These examples can easily find similar processes across the region when assessing the transformation that both Argentina's and Brazil's railways have gone through, or the reshaping of the entire existing assets operating on the Parana-Paraguay waterways along

\footnotetext{
${ }^{25}$ Cartelized ocean carriers adopting a common pricing policy for a given market anywhere in the world.

${ }^{26}$ A mechanism by which shipowners could flag off some vessels from their fleets while improving the utilization or load factors on the remaining ships on service by sharing their use with former competitors. Thus, both capital costs and operating costs tended to lower while performance indicators tended to raise.
} 
2,400 km of navigable waters and river terminals, or the changing trends in terms of air-cargo station hubs across the region, as well as the various land-bridges that evolved as a response to create high specialization as a strategy to counteract the offensive represented by the globalization players.

The theory of the firm provides a useful tool to lead the reader to gradually understand the existing motivations behind the Corridor's creation, and the way organizations find their ways to capitalize their experiences and project new possible scenarios to face the upcoming globalization and other economic and political trends indicated in Figure 3.1. The processes adopted by the Corridor in how it operates - further to including aspects that will be developed throughout other chapters such as economies of size, technological economies, managerial economies, operation and expansion economies, and diversification economics - triggered a number of alliances and partnerships of different degrees. These combined with a series of vertical and horizontal integrations and linkages within their respective supply chains, leads us to a fuller understanding of the Corridor as an innovative entity full of potential to be implemented under project management parameters in many parts of the world, as a way to generate larger economies of scale and getting corporations more competitive.

\section{5 - LEADERSHIP AND STRATEGY THEORY TO MEET STAKEHOLDER NEEDS}

Before discussing leadership in this section starts it is important to link to the purpose of business transformations explored in this thesis. In section 4.4 the concept of the corridor as a value chain is discussed. In that section and in previous and subsequent sections I talk about 'value' and 'benefits' to 'stakeholders'. This then, is an appropriate place to explain who I am referring to as 'stakeholder' and what I see as 'benefits' and 'value' that the Corridor project facilitates.

The literature has more recently expanded the range of stakeholders, and the stakeholder concept in terms of PM. Turner (1999) and Cleland and Ireland (2002) provide PM perceptions of stakeholders drawing upon earlier management literature. A chapter devoted in a recent PM book (Walker, Bourne and Rowlinson, 2008), provides a convenient summary of the term stakeholder as it has been developed from its roots in general management theory. They claim that "social science stakeholder theory tends to focus around concepts of justice, equity and social rights 
having a major impact on the way that stakeholder's exert moral suasion over project development or change initiatives" (Walker et al., 2008, pp71-72) and they cite the works of (Gibson, 2000) to support their view. They then discuss the instrumental stakeholder theory view as put forward by Donaldson and Preston (1995) where stakeholders are pragmatic demanders of some needs or wants that they are willing to negotiate about with the providers of those benefits. This can be complimented by the convergent stakeholder view (Jones and Wicks, 1999) in which sees stakeholders as those with legitimate moral needs to be considered as well as those who have the power and influence to instrumentally affect a project. Thus my perspective of stakeholders in this thesis is more in line with the convergent view even though many of the Corridor players tended to take a more instrumental view of who were legitimate stakeholders.

For the purposes of this thesis, a stakeholder is considered to have a legitimate stake in a project (in this case the business transformation) both from an ethical or business sustainability point of view as well as from a pragmatic view point in surviving the globalization wave that triggered the Corridor's birth as well as those conditions that persuaded players to join and participate in JVs such as that illustrated by case study examples. The stake is an interest representing investment in money, time, management and leadership energy as well as knowledge that is co-generated, transferred and used to create value and benefits. Value will be discussed in more detail in Section 4.4 but it can be seen here in shorthand as creating effectiveness (cost reductions) and efficiency (doing things smarter to gain more with less) as outlined in section 3.2 to 3.4. A benefit is described by Cooke-Davies (2002, p187) as the outcome from a project that fulfills the need and objects for initiating the project. Anderson, Narus and van Rossum (2006) use the term value proposition to describe how various stakeholders may have a need or value they wish to obtain by be answered the question why should a customer bother with our offering as opposed to our competitors. They argue that this requires a deep and detailed understanding of the customer's requirements and preferences. Suppliers can stress points of difference that they can deliver but still offer relatively little value to the target customer without ensuring that these customers actually place sufficient value on that offering to make differentiation worthwhile.

Leadership, and particularly leadership within the Corridor projects where JVs and other forms of collaboration feature, requires understanding both existing realities and potential futures. This 
will be discussed later in this section in terms of the Courtney, Kirkland and Viguerie (1997) preferred futures strategic thinking. The concept of benefits and being customer focussed with 'value' in mind turned out useful for the cases discussed in this thesis. This is because Corridor players who participated in JVs were concerned to meet the needs of their clients, customers and staff so that they could offer a value proposition that met (often as a JV in a form of business cooperation as discussed in Section 4.3) or exceeded their rivals outside the Corridor. Also, the focus on benefit turns out to be relevant to the creation and development of trust and commitment in the collaborations that the Corridor facilitated. A brief paragraph on this is salient here.

Trust is a concept that Mayer, Davis and Schoorman (1995, p715) explain as having four antecedents. One is the propensity of a party to trust. This means that for trust to exist, one party must be open to trusting the other and willing to test that trust. The three main factors affecting trust that they identify is benevolence (that is providing benefits and good will towards the other parties), having integrity (that is being consistent in the way that they do what they commit to honestly and openly) and having the ability to do what they say (as individuals as well as being able to deliver within the systems that they operate). These three factors are continually tested by a party that is willing to trust and positive results reinforces and builds trust while negative experiences detracts from the 'bank' of trust. The Corridor's role as mediator and facilitator helped this trust to be established which, as discussed in Section 3.7, also affects the effectiveness of knowledge development and use. Further, trust has an impact on commitment.

Meyer and Allen (1991) define commitment at three levels each with different implications. People may be compliant which really expresses little if any commitment other than do what the law or contract requires, this may be considered as not being committed but compliant. The lowest level of commitment relates to what Meyer and Allen (1991) call continuance commitment where there is a willingness to do something because not doing will result in harm to that person, often financial harm or some form of lower level of benefit received. The next level is called normative commitment where the person feels obliged to do what was promised or committed to. This could be seen as begrudging commitment or simply doing the minimum that norms require. The most powerful form of commitment is affective commitment where goals, objectives, vision and desire are matched so that the person wants to do what they committed to. 
So we can see from the above that if stakeholders (be they players, employees, clients or customers) who have an interest in a business cooperation of the kind investigated in this thesis, have high levels of trust and high levels of effective commitment, then the outcome is likely to be favourable. The aim of leadership, from this stakeholder perspective, is therefore to ensure that barriers to this state are reduced and that drivers are designed to be put in place to enhance and enable this kind of trust and commitment to flourish. This leads to a discussion about leadership style and approaches that favors or hinders the working environment that affects trust and commitment.

The concept of situational leadership has been with us for a long time (Tannenbaum and Schmidt, 1958; Hersey and Blanchard, 1982; Hersey, Blanchard and Johnson, 1996). It is generally accepted that leadership may be a waste of resources and definitely counterproductive in certain situations in which the environment does not call for a forceful leader but where sound and good management maybe through shared understanding and agreement on action to be undertaken is the minimum needed to face the kind of situation where contextual volatility and uncertainty prevail.

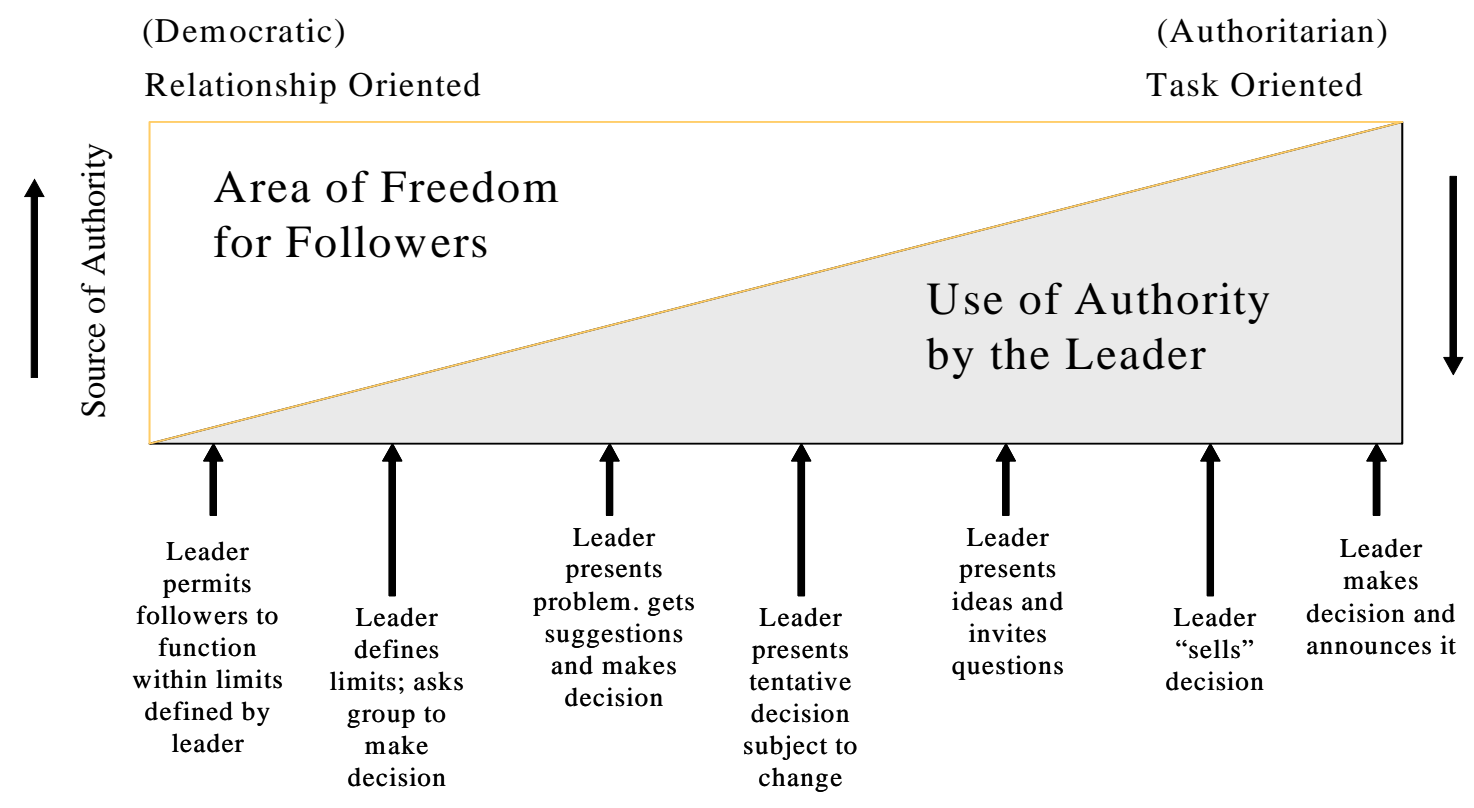

Figure 3.2 Authority Sources and Leadership Style - Adapted from Tannenbaum and Schmidt $(1958$, p96) 
While the leadership literature has progressed a long way over the past 50 or 60 years it is worth reviewing some of the formative ideas about leadership because a leadership mentality has a strong impact on the way in which any organization, including the Corridor, functions. Figure 3.2 illustrates the range of leadership styles observed in practice that spans a highly authoritarian approach to a pure collaborative and democratic approach. The background logic behind these styles was explored, researched and results described by Hersey and Blanchard (1982; Hersey et al., 1996) and this model is presented in Figure 3.3.

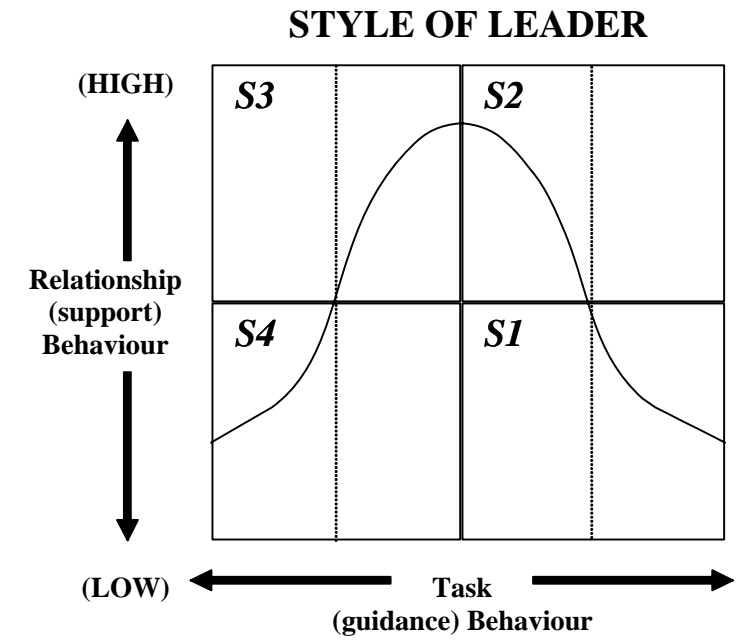

S1 = Provide specific instructions \& closely supervise performance S2 = Explain your decisions \& provide opportunity for clarification S3 = Share ideas \& facilitate in making decisions S4 = Turn over responsibility for decisions and implementation

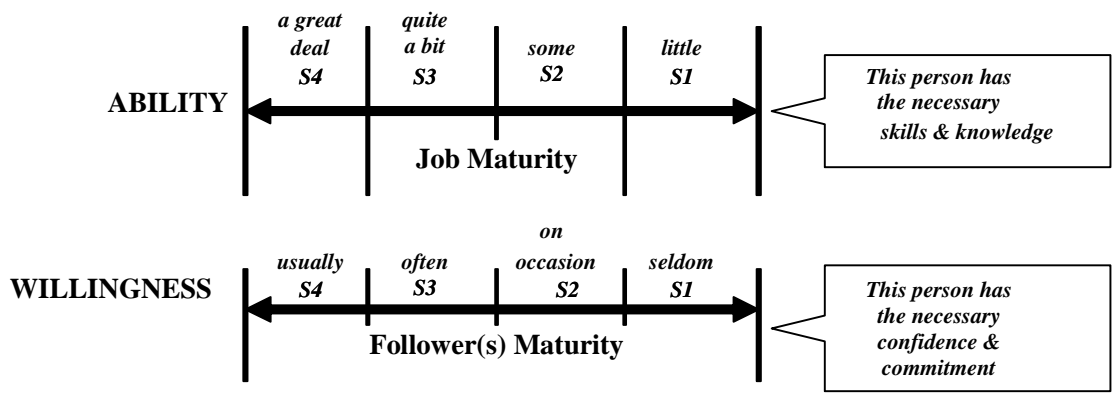

Figure 3.3 Leadership Styles and Follower Maturity: Adapted from (Hersey et al., 1996, p208)

Figure 3.3 introduces follower maturity or the ability of them two participate in the decision making process based on their willingness and ability to contribute. The four styles and represented along the continuum in Figure 3.3 and explain the styles in terms of four styles S1 to S4. Style S1 and S2 are directive relying on more transactional motivation whereas style S3 and S4 have opportunities for transactional motivation, they rely on the follower's intrinsic 
motivation to act as is necessary. Transactional refers to there being an exchange of some sort of either tangible rewards such as pay and remuneration or perhaps more intangible rewards such praise and encourage a sense of self-worth.

Transactional leaders motivate subordinates to perform as expected. They do this by helping them recognize task responsibilities, identify goals, acquire confidence about desired performance levels, and understand that their needs and the rewards desired are linked to goal achievement (Bass \& Avolio 1994, pp13-19). Transformational leaders motivate individuals to perform beyond expectations by inspiring them to: focus on broader missions transcending their own immediate self-interest; concentrate on intrinsic higher-level goals (achievement and selfactualization) and not lower-level goals (safety and security) and be confident in their abilities to achieve the missions given by the leader.

Probably, a truly transformational leader is that one that becomes transactional for a while. In this connection Bryan and Hulme (2003) - directors in McKinsey's New York and Houston offices respectively - argue that companies can find additional earnings in two ways: they can try to improve operating performance by squeezing more profit out of existing capabilities, or they can improve corporate performance by organizing in new ways to develop initiatives that could generate new earnings. By pursuing both of these approaches simultaneously, companies can take a powerful organizational step toward meeting the challenges of today's hypercompetitive global economy. Consequently, the border between transactional and transformational leadership seems to be extremely thin or at least overlapping in the best of cases, the context being the main driving element to blend these two leadership styles.

Some writers (e.g., Bennis \& Nanus 1985 ; Zaleznik 1998, pp62-70) contend that leadership and management are qualitatively different and mutually exclusive, or put in other words, that management and leadership cannot occur in the same person . In other words, some people are managers and other people are leaders. Yukl (2002 pp5-6) sustains that managers value stability, order, and efficiency, whereas leaders value flexibility, innovation, and adaptation. Managers are concerned about how things get done, and they try to get people to perform better. Leaders are concerned with what things mean to people, and they try to get people to agree about the most 
important things to be done. Bass \& Stogdill (1990 pp383-86) are of the opinion that transformational leadership does not substitute for transactional leadership. It supplements for it with an add-on effect, performance above expectations. The logic is that the most successful transformational leaders need transactional skills to manage effectively the day-to-day events.

Authors cited above, who refer to transformational and transactional leadership style dimensions seem to hold varying views on how intensely the context applies to these two leader-follower style relationships. However, these two leadership style dimensions mutate and gradually shift in intensity between these two poles on the dimension in accordance with the various roles companies had to play within the Corridor. All of these companies found themselves subjected to volatile regional circumstances as global trends exerted significant pressure on them to adopt their leadership styles.

Another vision of leadership is provided by David (2003 pp157-185) who argues that a cost leadership strategy will rarely call for a leader but a skilled manager capable to trace the slightest cost deviation and act accordingly. Leadership, by contrast, is about coping with change (Kotter 1998, p40) - this possibly being the main constituting driver of the Corridor project itself. David (2003) further argues that a differentiation strategy, for example, is more likely to demand a leader whereas a divestiture - purely transactional - may better require a manager.

Both mean change though each encompasses a different leadership dimension: clearly the former calls for a transformational style while the latter results more transactional - though probably not entirely transactional. However, the size of the firm and its global positioning also play a role in turning a transactional leader into transformational, or a transformational attitude into a much more cautious and conservative transactional leadership style. This is been precisely the changing and volatile nature of the South American market where the ever mutating business environment seemed having empowered some players while limiting others, rather than having created the conditions for leaders to turn up and develop naturally while formulating and implementing their previously elaborated strategies. 
Economies of scale, global positioning, financial back-up, and knowledge capturing seem having all played a key role in making the regional players react and set up the Corridor organization as a way to offset the globalization impact on their businesses. Courtney, Kirkland and Viguerie (1999 pp5-30) who are consultants at McKinsey \& Company argue that uncertainty, a concept which turns out abundant in the region, requires a new way of thinking about strategy. They say executives take a binary view: either they underestimate uncertainty to come up with the forecasts required by the firm's planning, or they overestimate it, abandon all analysis and go with their gut instinct. This "just do it" approach to strategy can make executives face extraordinary surprises for which were not trained to overcome or even think about it. The environment into which leaders and followers have to act can never be changed but that at best they can intend adapting to it and try to make the best within the given constraints and limitations - either operational, financial or human (Cleland \& Ireland 2002, pp455-64).

Further to Porter's (1985) generic strategies, there are a number of strategies that were adopted by a great number of players within the Corridor structure. The global newcomers also adopt similar strategies to the regional firms (though using a different leadership positioning perspective) in their pursuit of enlarging economies of scale and expanding markets, though this was contrary to what happened within the Corridor, as it entailed a rather transactional leadership style that tended to be facilitated by the plentiful resources available for these firms to deploy.

Typically, vertical integration strategies were common on both sides - those who were reacting defensively before the globalization process, as well as those who were protagonist of such an expansionist trend. Vertical integration strategies allow a firm to gain control over distributors, suppliers, and/or competitors. Forward integration within the value/supply chain turned out to be common as it proved useful in gaining ownership or increased control over distributors. Ocean shipping companies as well as railway companies tended to integrate trucking operators and offdock stations ${ }^{27}$ all over the region aiming at limiting customers on their choice to pick up an independent service. Port terminals all over the Atlantic coast not only acquired entire trucking companies, but also integrated cargo surveying firms and shipping agencies as a way to have a

\footnotetext{
${ }^{27}$ Warehouses located outside the port terminal area where a number of exporting support activities are carried out (e.g. stuffing of containers ; stacking of containers ; cargo surveys ; customs clearance ; cargo storage; cargo lashing ; lumber fumigation; etc) and whose control may help improving a given market share.
} 
direct influence on both shippers and ocean transportation companies. Shippers would rather prefer contracting a single party with whom the whole circuit could be set forth, whereas shipping companies would see the opportunity to offer more services and therefore inducing shippers to utilize their services without incurring in heavy investments. Backward integration played an important role in that a great number of suppliers were practically swallowed by both global and regional operators who aim to hold better and tighter control over the destiny of the cargo flows all across the region.

Vertical integration strategy was a very common result with respect to shipping companies: acquiring those firms that used to render shipping agency services as well as tug-boat operators; empty container yards ${ }^{28}$; ship chandlers; and logistic operators controlling a given market segment or number of customers, and even port terminal services. Air-cargo firms also tended to rapidly acquire their contracted air-station depots instead of outsourcing them, feeding trucking services $^{29}$, and third-party sales agencies as a way to hold better control on the market. River barging companies and coastal feeding operators were either absorbed by larger and global operators in their condition of potential suppliers, or went for shopping themselves by integrating their own suppliers such as river terminals, naval repair facilities, inland distribution centers, etc.

Horizontal integration strategies also played a fundamental role in the form of mergers, acquisitions, and takeovers among competitors, and as a way to conduct a growth strategy leading to achieving larger economies of scale and enhancing the transfer of resources and competencies. Davidson (1987) argues that mergers between direct competitors are more likely to create efficiencies than mergers between unrelated businesses, both because there is a greater potential for eliminating duplicate facilities and because the management of the acquiring firm is more likely to understand the business of the target. Typically, this is been the situation with a number of global shipping companies taking over the regional ones, as well as global port terminal operators who acquired a number of both ocean and river terminals.

\footnotetext{
${ }^{28}$ Yards where entire fleets of empty containers are stored, maintained, repaired, and made good for the exporter to pick up and deliver alongside ship to initiate the ocean transportation leg.

${ }^{29}$ Van and truck services linking inland origins with air-stations.
} 
Krass (2001) contends that a reason why companies still merge, even in the face of odds against success, is the fact that investors and shareholders greatly reward increased market share and geographic expansion. Krass (2001) further highlights the fact that future growth expectations account for more than 60 percent of an average company's market value today, up from about $40 \%$ a decade ago. David (2003, p37) helps explain this business expansion in terms of market penetration, market development, and product development—sometimes referred to as competitive intensive strategies because they require intensive efforts if a firm's competitive position where existing products or services are improved.

Market penetration strategies were adopted by most of the global operators in almost every single segment of activity within the logistic field in view that markets were not saturated at the outset of the globalization process, enlarged economies of scale were regarded as a key variable towards achieving major competitive advantage, and the sense that loading factors could be increased significantly. ${ }^{30}$ A market development strategy was perhaps the main driving strategy behind global shipping companies and port operators, as well as global logistic firms and railway operators - among many others - towards enlarging economies of scale and gaining competitive advantage. Naturally, to do so demanded a successful organizational structure, new untapped or unsaturated markets, plenty of capital and human resources availability, an existing excess of supply or sub-utilized operational capacity, and the very perception that the company's core business was becoming rapidly global in scope. This action on the part of the global firms made room to a reaction on the part of the regional companies that regardless of their activity segments, their market share, geographical scope, and available assets, tended to get together in a defensive fashion leading to the creation of the MERCOSUR Atlantic Corridor. ${ }^{31}$ The only exception to this rule is given by the barging business all along the 2,400 km Parana-Paraguay waterways and the Amazon River in Brazil, in view of the existing traditional knowledge which proved being an

\footnotetext{
${ }^{30}$ Loading factor is the rate of utilization of a vessel's carrying capacity. The available space on the main liners sailing east-west-east were aimed at being reduced or its effective utilization increased, in view of the geographical expansion toward north-south-north trades where they were absent before the expansion process begun. The main liners tended to reach better results by capturing more cargoes destined to remote location by transshipping same on to feeding vessels southbound and northbound.

${ }^{31}$ Most of the global carriers regularly extended their services to south America (Maersk/P\&O/Sea-Land/CP Ships/Hapag-Lloyd/Evergreen/Cosco/MSC) as well as a great number of port terminal operators (P\&O Ports/ICTSI/Maersk/Hutchinson).
} 
unsurpassable barrier to the global newcomers - as it will be dealt with in chapter 7 where a case study on this topic will be presented and analyzed.

Product (or service) development strategies were mostly undertaken by the port terminal operators by making available to customers new technological devices for on-line cargo tracking, and by installing efficiency enhancers GPS ${ }^{32}$ devices - further to the general betterment of the existing operational facilities leading to service improvement and cost lowering.

David (1985, pp14-17) defines five guidelines when a service development strategy may be an especially effective strategy to pursue:

1. When an organization has successful services that are in the maturity stage of its life cycle; the idea here is to attract satisfied customers to try new and improved services as a results of their positive experience with the firm's present services;

2. When an organization competes in an industry that is characterized by rapid technological developments;

3. When major competitors offer better-quality products at comparable prices;

4. When an organization competes in a high-growth industry; and

5. When an organization has especially strong research and development capabilities.

National owners of port terminals had to either quickly reengineer their businesses or give up and sell out. This becomes apparent in view of several changing trends that had remained entrenched right up to the point that the globalization wave arrived to the region that. These changes that operators were confronted with entailed moving from an environment where the economy was on the verge of market protectionism; the existence of a rather paternalist leadership style across the region and the presence of flag-protection schemes ${ }^{33}$.

\footnotetext{
${ }^{32}$ Global Positioning System displays the exact physical location or geographical coordinates a given container has at a given moment. It renders both the customer and the port operator a permanent follow-up of a container routing and therefore facilitates both to estimate timing of cargo delivery and container drop-off.

${ }^{33}$ Cargo restriction laws that were applied for Argentine flag ships carrying imports into the country, or for Brazilian flag ships trading in and out of Brazil - further to numerous bilateral treaties which tended to support the utilization of their respective fleets.
} 
A similar process did happen in the railroad sector which unlike the port business, was acquired first by strong national firms who at a later stage saw themselves in the position of having to sell out to even stronger and more capable regional groups. These groups were precisely in search of enlarging their economies of scale to accompany the process or face the impact of the upcoming globalization while achieving lower relative operational costs. Diversification strategies did not play here a fundamental role as most global players kept their focus on what they did best as defined by their respective core businesses.

Concentric diversification strategies were, however, actively adopted through the Corridor networking as a defensive reaction to face what Muto (2001) refers to as six useful guidelines to seriously consider concentric diversification strategies:

1. When a firm competes in a no-growth or slow-growth industry;

2. When new, but related, services could be offered at highly competitive rates;

3. When adding new, but related services, would significantly enhance the sales of current services;

4. When new, but related, services have seasonal sales levels that counterbalance an organization's existing peaks and valleys;

5. When services have entered into the declining stage of its life cycle;

6. When an organization has a strong management team.

These types of strategies were often adopted by those national and regional logistic operators that saw their income sources or customer-base threatened by the arrival of global newcomers. They went to the crating, fumigation, lashing, and packaging business in an attempt to offset their decline in sales. Other example is given by those independent trucking firms who appointed regional and even overseas representatives in their intent on controlling import cargo flows which otherwise would have been easily routed through someone else's organization.

River terminals also diversified by providing cargo surveying and customs clearance services at the shippers' premises, further to supplying lashing/packing material and hiring lifting equipment by request. National custom brokerage firms went regional and even overseas by offering their professional services and by expanding into logistics too, as a way to ensure demand for their 
core activities. Ship-brokers opened up their often small and local organizations and also went regional by offering ancillary services such as warehousing, trucking, surveying, security, and even claim management. National shipyards also went regional further to diversifying into new lines of production (barge shipyards expanded into rail wagons; fishing vessels shipyards also started building barges and tugboats; ocean-going full-container shipyards tended to build smaller coastal feeders and also ocean tankers). Classification societies ${ }^{34}$ tended to open branches on global logistics, not as a defensive reaction but as a way to take advantage of their already global structure in the classification business for the ship-building, oil and gas, mining, and construction industries.

It becomes apparent that in view of the uncertainty factor that is yet present in South America and which arises out of an ever-dynamic and volatile business environment, it seems very unlikely to typify a single or unique leadership style that matches a Corridor-like entity - along with a single associated strategy type. Leadership and strategy seem to take many different forms within the Corridor in view of its members' diversity and their different business realities and potentialities they have been obliged to face.

Naturally, other variables within the human resources, knowledge, and operations dimension, also play a role when trying to identify the dominant leadership and strategy styles. Some examples may help the reader visualize some of the variables that will guide us through the dissertation towards better comprehend why leadership and strategy find so many different forms. Markides (1998 p88) contends that too many companies mistakenly assume that they can break up clusters of competencies or skill that, in fact, work only because they are together, reinforcing one another in a particular competitive context.

Hamel and Prahalad (1994 pp49-54) refer to the importance for corporations to develop and strengthen their organizational culture through engendering a rich genetic coding policy where beliefs, values, and norms, support different views or contrasting perspectives and facilitate companies therefore to be better placed to face a troublesome or changing business environment.

\footnotetext{
${ }^{34}$ Global organizations such as SGS, BUREAU VERITAS, DET NORSKE VERITAS, AMERICAN BUREAU OF SHIPING, GERMANISCHER LLOYD, NKK, who provide surveying and classification services to a wide array of industries.
} 
Considering adopting an appropriate leadership style became essential when, for example, outsourcing policies were formulated and implemented as a result of the need for the Corridor companies, to catch up with the global trends and remain competitive. Auguste Hao, Singer and Wiegand (2002) argue that outsourcing often results in being hostage to providers who could be tempted to raise prices and reduce services.

The concept of transaction cost economics is useful for us to understand what was happening and triggering the activities within the Corridor. Oliver Williamson (1985) is one of the most widely cited authorities on transaction cost economics (TCE) which essentially means the cost of transacting business. This includes the cost of tendering, the cost of administering contracts and the cost of maintaining relationship to mention just a few of the 'hidden costs' involved in doing business. Often an apparent saving in one area merely adds to costs in another for example outsourcing to save direct costs can incur contract administration costs and strategically loose an organization a strategic advantage to expand their business in a particular way.

Ghoshal (1996) argues that TCE helps explain why customers and providers alike often find themselves saddled with increasing transaction costs (such as managing the relationship; monitoring the delivery of services; and coordinating the exchange of information between customer and provider) that had not been anticipated and sometimes exceeded whatever value one or the other party had hoped to gain. We see various examples of this concept across the global players that imposed their services without sometimes having measured the actual extent.

Auguste, et al. (2002, p55) argues that "in the long run, the providers must realize not only economies of scale but also economies of skill - by codifying the process innovations developed while serving one customer so that they can be used in work for other customers”. This suggests that outsourcing within the Corridor provides a fertile ground to study the type of leadership arising out of an ever changing business context. Furthermore, strategic alliances, partnerships, joint-ventures, mergers and acquisitions will be further explored within a knowledge management (KM) and leadership perspective. 


\section{6 - BUSINESS COOPERATION THEORY}

An element that was present by the time the Corridor project tended to materialize that is currently present is the uncertainty factor. In the face of increased uncertainty, a company can choose between spinning off or internalizing activities. Typically, entering a new geographic market as a spin-off company is a decision involving a high degree of uncertainty caused by a number of factors: difficulty in quickly identifying customers’ preferences; lack of knowledge about competitors; market response to the service; potential cultural barriers and so on. In this situation, the establishment of agreements such as joint ventures or a range of alliances enables participants being able to rely on more information and reducing the existing uncertainty levels to a more manageable dimension. This is been an option that not only outsiders to the MERCOSUR Atlantic Corridor have taken in order to enlarge economies of scale and gain competitive advantage, but also the regional firms by adopting some sort of defensive reaction within a market that they have been active in for a long time and under moderate competition.

Internalizing - the other option - means the acquisition by fusion or takeovers of local firms as a means of entering these markets. In these markets, where volume is a critical variable, greater presence or concentration is a fundamental competitive factor. Larger size and therefore greater economies of scale can be achieved by internal or external growth based on acquiring more control over a given market. Internal growth implies increased administration and management costs, whereas external growth, management and organizational costs may tend to increase owing to the uncertainty generated by the acquisition process itself.

Cooperation between companies represents a third option to achieve larger size - though it also involves certain costs derived from organizing and put into practice the cooperation agreement. Arranz Pena and Fernandez (2002 p21) argue that the reason for company cooperation can be summed up as follows:

- Reducing transaction costs that arise when uncertainty increases;

- Obtaining a greater volume and presence in the market by reaching agreements with competitors, suppliers, or customers; 
- Seeking efficiency in certain activities carried out by the company by means of spinning off, when the internal costs of carrying out the activity are greater than if this activity is carried out in the market with logical competition; and

- Using specific assets which the firm does not possess, but which complement its activities. According to the specificity of assets, cooperation may prove more efficient than acquisition in the market, since uncertainty is reduced on obtaining them without incurring any internalization costs.

James (1985), on pointing out that the fundamental aim of a military - or economic - alliance is either to combine participants' potential or effective forces so as to increase the latter with respect to adversaries, or to dissuade potential opponents from committing themselves to a future conflict, states intuitively the main aims that might be pursued in cooperation: by pooling, combining or exchanging resources it attempts to enable partners to gain access to advantages they could not achieve individually.

A business cooperation phenomena generally includes different forms of association concentrating on internationalization strategies, technological innovation or market expansion, for example. The two most common explanations for cooperation are: the search for growth or the power of the market; and the search for synergies or complementariness. The first of these and according to Kogut (1988 pp319-32), rests on the competitive positioning and industrial economy theories. If maximizing an activity's profits depends on improving the company's competitive position with respect to rivals, therefore, and if the necessary resources or risk acquired exceed its resources, cooperative association would enable economies of scale, experience and risk diversification to be obtained, while at the same time increasing the power of associated companies within the sector. This type of cooperation associates firms that might be comparable within a connected field and with identical problems, representing the common link for resources of the same type.

The second case is based on the synergies obtained by grouping or combining qualitatively complementary assets and this also fits in well with the RBV of the firm. Adler (1979 pp58-98) defines it as symbiotic or differentiated cooperation where complementary companies get 
associated aiming at pooling or exchanging resources - each contributing different qualities. Naturally, this combination of forces enables a more complete or intense use of the different types of assets possessed by each firm in unequal proportions.

Collins and Doorley (1992) contend that a series of advantages can generally be identified for companies, both with respect to similarity cooperation (comparable organizations with identical problems that seek greater size), as well as in the case of complementary or synergic company associations (combining resources to use different types of asset more completely). These advantages include:

- increased capacity and competence without needing to acquire and develop new resources and abilities;

- time gained with respect to competitors; and

- company flexibility is maintained, which is very necessary for adapting to the changing environment in which their activities generally take place.

Naturally, cooperation agreements often are associated with a number of disadvantages (Collins and Doorley1992):

- A reduction in the associated company's strategic autonomy that must accept a redistribution of order and control in exchange for the advantages sought - which often creates mistrust on the part of the executives and directors;

- The need to harmonize and coordinate the decisions and actions of two or more independent organizations, often with different structures, systems and even cultures, which can lead to conflicts between associated firms or to a possible obstruction to the activity that is object of the cooperation (this can lead to a conflicts between associated firms or to a possible obstruction to the activity that is object of the cooperation which would lead to a gradual deterioration in each partner's overall performance); and

- It can dissipate the strategic advantages of a company by means of the learning of its technology by a third party, creating a new competitor or fortifying an existing one, when sharing technology and knowledge with the partners. 
There are three environmental factors that can be regarded as having an impact on company structure and which inevitably led many regional companies to getting together and undertake a number of sub-projects within the Corridor - a master project in itself. The first factor is given by economic globalization and along with it the liberalization of capital movements in international financial markets, all representing a new reality that even exceeds the capacity to react before potential financial problems (e.g. financial crisis in Mexico, Brazil, Russia, Japan, and Argentina).

In this increasingly inter-dependent world, companies have to face new competitive challenges such as the appearance and entry of international-scaled competitors, the international expansion of markets, and greater innovation capacity. Davidow and Malone (1992) argue that in being faced with this great inter-dependence between economies - economic globalization - companies are responding with a less localized view and tend to adopt and adapt both a more global perspective and more versatile organizational structures.

The second factor is given by the increase in business uncertainty that arises out of - to a great extent - the mentioned inter-dependence concept. The speed with which changes are occurring on global basis in the economic world and get transmitted across regions and countries, introduces great uncertainty. This is especially true for those activities that demand constant technological transformations and market follow-up to keep pace of the new surrounding conditions - established by both companies struggling for market expansion and customers demanding better or more integral services. The uncertainty of the environment also places greater demands on firms' speed of reaction to adjust to transformations (their reflexivity), or even their intention to predict them, something that derives from the need to design a flexible and adaptable organization.

The third feature characterizing the business environment is the high level of competitive rivalry. Increasing customer requirements and market saturation are variables that oblige companies to constantly dig deeper in their search for competitive advantage and market positioning. As a result of this, there is a tendency for companies to concentrate on know-how, or on those aspects of their added value chain they really dominate. This greater level of competitive rivalry is, in 
turn, producing a regrouping or concentration in those activities where competitive advantage exists and therefore typically leading to adopting a downsizing, rightsizing, leaning, or reengineering strategy.

Handy (1992) contends that organizations seeking greater flexibility are eliminating internal barriers and hierarchies, reducing their size and forming a complex of autonomous business units that are aimed at the market and linked horizontally. These structures are setting up new forms of organization by means of internal cooperation, constituting the so-called internal cooperation or intra-company network. On the one hand, companies are introducing greater flexibility by creating autonomous units and, on the other hand, they are increasing their presence in other markets - globalization - by concluding cooperation agreements with other firms. Greater cooperation between companies is also driven by the advances of information technology. In this regard, Davenport (1993) argues that technology is not only introducing substantial advantages, but also enables geographically remote locations to be connected, communications to be established practically in real time and therefore, it eases decentralization of information and transmission via communication networks - all concepts that turn out key in international logistics. Technological leadership can be considered as probably the most important factor making regional firms approaching each other and global firms getting together to set a cooperation path that might eventually lead them to enlarge their economies of scale and step into new markets.

The Corridor project in itself can be regarded as a cooperation agreement which was born as a consequence of two other elements that often cause cooperation to work: market interdependence and uncertainty. These two variables when working together seem to have brought about the necessary environment for companies to get together, exchange their experiences by articulating and socializing knowledge, and give birth to a number of sub-projects that may well find correlation with one of the various types of cooperation agreements.

Porter et al. (1987, pp437-51) define cooperation as verbal or written agreements concluded between various companies in order to carry out an activity jointly, with certain definite objectives and a certain degree of coordination and structure in which to carry them out - with 
their duration depending on the aims being pursued. This definition opens a number of possible strategic TCE variants as per Williamson (1975):

- Independence and redirection strategies, where the aim is to reduce uncertainty and dependence on the surrounding environment, by exploiting the associated firms' own resources and competitive advantages;

- Cooperation strategies, which involves agreements between competitors, either on a general scale or in specific activities, the aim being to reduce temporarily the existing competition between them;

- Linked relations or delegated link strategies, which are the result of one company's agreement with another - the delegated or subcontracted one- in order to have a task, activity, or function carried out on their behalf instead of assuming it themselves; and

- Alliance strategies, which are agreements that attempt to modify the competitive environment, to eliminate or moderate competition between a number of players for a given period of time. From the various kinds of alliance, a cooperation agreement might not be explicit - contrary to the more formal alliance-type agreements such as joint-ventures and partnerships which should be explicit.

Even though the Corridor project witnessed a great variety of sub-projects developed and completed under its auspices, a great majority of the interactions first went through a collaborative dimension to later engage in some sort of explicit commitment under an alliance type of cooperation. The above suggests that regardless of how uncertain or intertwined markets may look, some sort of collaborative stage needs first to be overcome in order to let the way open for companies to achieve more formal and wider agreements. This dissertation will focus more on those sub-projects that based their agreements straight on a formal basis, though which experienced a great deal of cooperation during the formation phase - without which these undertakings would not have been feasible in the first place.

\section{7 - KNOWLEDGE MANAGEMENT AND SITUATED LEARNING THEORY}

When markets shift, technologies proliferate, competitors multiply, and products become obsolete almost overnight, successful companies are those that consistently create new knowledge, disseminate it widely throughout the organization, and quickly embody it in new 
technologies and products. These activities make up the "knowledge-creating" company where its sole business is continuous innovation (Nonaka, 1991; Nonaka and Takeuchi, 1995; Nonaka and Teece, 2001). According to Nonaka (1991) successful Japanese competitors like Honda, Canon, Matsushita, NEC, Sharp have become famous for their ability to respond quickly to customers, create new markets, rapidly develop new products, and dominate emergent technologies - the secret of their success being the way knowledge creation was managed. Even though it seems quite unlikely that innovation may occur almost overnight in terms of logistic services, the above description looks very appropriate to underline the importance of knowledge in a competitive environment.

Much of the analysis of this thesis and its premise as based upon research question 2, relates to knowledge creation, sharing/transfer and use by Corridor participants. Therefore associated literature is highly relevant that helps explain how and why individuals and organizations create and share knowledge to co-create value and competitive advantage

Davenport and Prusak (2000) argue that the KM concept holds links with a number of various aspects of the business organization. Zack (1999) shows us how KM links with business strategy in that knowledge is an asset that may become represented by pricing knowledge, research and development, promotion knowledge, customer knowledge, or market knowledge. These authors found that it is not actually very difficult to envision ways of using knowledge more effectively in business strategy, but that the difficulty lies in actually making the changes to strategic programs and adopting the necessary behaviors throughout the organization.

KM has to do with work processes in that how market researchers, scientists, consultants, engineers, and executives create, gather, store, share, and apply knowledge on their daily tasks. While knowledge workers may view their work in unstructured terms, it is possible to view knowledge work as a somewhat structured process that can be designed and improved. Markus (2001) argues that knowledge processes are often categorized by whether they involve knowledge creation (as in research or new product development) or knowledge reuse (as in sharing best practices or helping others solve common technical problems). It has to do also with culture and behavior in that how an organization can best create a culture and set of behaviors 
that value the creation, sharing, and use of knowledge, and consider said concepts as long-term achievable goals. The central problem here is probably given by the management of attention or the proper understanding of the way it is allocated by individuals and organizations, knowing how to capture it more effectively, and using the technology both to acquire and protect it. Finally, it also has to do with the physical business environment, an issue one might suspect being of minor importance but that can be a pivotal factor to ease or foster knowledge creation and sharing.

Nonaka (1991) distinguishes between two types of knowledge: explicit and tacit. The former is formal and systematic, easy to communicate and share in the form of a product specification, scientific formula, or computer program. The latter turns out highly personal and therefore hard to formalize and communicate to others - further to containing the very personal skills that a determined job, profession or technology may demand. This author highlights four basic patterns that bring about knowledge creation, known as the SECI cycle:

1. Socialization -Tacit to tacit occurs when an individual shares tacit knowledge directly with another and therefore creates knowledge under a sort of "socialization" process. This may result in a very powerful way to create knowledge provided that a sharing system is in place too. The simple attendance of the Corridor members to the roundtable discussions and presentations, do not guarantee nor represent a fertile ground in itself for companies to spontaneously share their tacit knowledge - unless a well design sharing mechanism deploying business safeguards is in place, along with a “strong motivation driven by the business context - as argued by (Sense, 2007)”.

2. Externalization - Explicit to explicit: occurs when an already existing knowledge transforms itself in a new piece of knowledge giving a better or deeper insight on a determined issue. This is not necessarily regarded as one of the most powerful means to create knowledge, though it may prove necessary for decision-making in business (access to a number of market ratios, financial or accounting information, or variables that may result useful for benchmarking purposes).

3. Combination - Tacit to explicit: occurs when articulating tacit knowledge into explicit knowledge that may bring about innovative ideas of cooperation. This was experienced within the Corridor among firms that were in search of not only achieving larger 
economies of scale, but also trying to capture a different view of the existing business reality that might help them survive and even grow while facing the globalization impact all over the region.

4. Internalization - Explicit to tacit: occurs when explicit data information and knowledge is reframed and made sense of and so becomes personalized and internalized. It was hypothesized as essential for knowledge creation provided that a sharing mechanism is also in place - this is what Nonaka and Konno (1998) refer to as $b a$ being translated from the Japanese word for 'place'. In this regard, the Corridor proved being an efficient forum through its roundtable events to let internalization of documents, reports, statistics and other pieces of information facilitate change and innovation.

Garvin (1998 pp48-78) contends that an organization cannot improve without first learning something new and that solving a problem, launching a service, or reengineering a process all require adopting a learning attitude on the part of both companies and individuals. He argues that organizations that systematically approach the management of institutional, organizational and personal knowledge in a way exemplified by the SECI process are acting as learning organization. Otherwise change remains cosmetic, and improvements are either fortuitous or short-lived.

A forum such as the Corridor was hypothesised as a good example of a knowledge organization in that it contains, from my observations, a number of knowledge-hungry organizations and individuals who for different reasons joined the Corridor entity in search of knowledge-based solutions that might help them face a changing business environment. Senge (1990, p1) describes learning organizations as places "where people continually expand their capacity to create the results they truly desire, where new and expansive patterns of thinking are nurtured, where collective aspiration is set free, and where people are continually learning how to learn together”. However, in South America and particularly, from my observations as a facilitator within the Corridor, this sort of learning attitude was not born "per se" or because of a sudden upsurge of a self-improvement feeling or vision on the part of the members; but that on the contrary, it was 
probably due to having to face the unknown that manifested itself in the form of global companies arriving in that part of the world.

I provide below, my observations and reflections upon evidence subsequently used as case study data that are useful for better understanding the relevance of many KM issues that I felt were salient to this study. The following reinforces the important of knowledge as a strategic competitive advantage asset that is often tacitly held by key individuals and in unstructured or implicit forms within organizations.

Such a sudden and perhaps violent chain of events that was hypothesized to be in all and every logistic segment along the MERCOSUR coasts and territories, was driven by a marked market saturation on the east-west-east trades on the part of the global ocean shipping companies, and the overwhelming excess of carrying capacity that the main ship-owners had to face in their permanent search of larger economies of scale.

The evolution of the economies of scale (as discussed earlier in the chapter from a strategy and competition theory perspective) drove a number of knowledge-based issues that are the focus of this section of the chapter. Sailing ships involves a great deal of both highly explicit knowledge (charts, weather reports, news reports about safety and risk conditions, stowage plans, loading and discharging computer-based modeling planning etc) as well highly tacit knowledge (likely impact of localized weather patterns, cultural knowledge applied to crew management, navigational decisions on speed, course deviations, ports or other facilities are more reliable as others in response to conditions of trade at any given time or 'rumors' of those who linked into informal social networks about a range of political and economic issues that have risk and uncertainly impacts).

The range of tacit knowledge in particular varies over time and by region because events are very fluid and knowledge about their causation and impact is often highly opaque. Thus the size of ships and their ability to travel along a particular route is closely linked to local knowledge. Also, much of so-called market intelligence is about gossip and as such is common knowledge and dyadic knowledge that is at the same time both explicit and tacit (through interpretation). 
Until 1988, the biggest container ships carried 5,000 TEUs. This meant that they were small enough to fit through the Panama Canal. Ships must be no wider than 32.3 meters, no longer than 294.1 meters, and have a draught no greater than 12 meters to cope with the locks along the 80 kilometer journey between the Atlantic and Pacific. Ships that fit he Panama Canal are known as Panamx class, whereas today's full-container ships are known as POST-Panamx class and have a capacity of up to 11,000 TEUs. According to Containerization International ${ }^{35}$, if the pursuit of economies of scale drives ship sizes beyond 18,000 TEUs, then nature presents another formidable obstacle. Vessels of this size are already known as MALACCAMAX class, meaning they would be the largest able to sail through the Strait of Malacca between the Malaysian Peninsula and the Indonesian island of Sumatra. This $805 \mathrm{~km}$ stretch is a shipping lane of equal importance to the Panama and Suez Canals though it presents a shallow point of 25 meters deep, limiting passing ship’s draught to 20 meters.

Barges transportation and shipping along the fluvial system of rivers and waterways between the borders of Brazil and Argentina for example (Parana-Paraguay waterways), presents similar knowledge management issues. Currents and rapid changes in water flow affects the capacity of goods to be transported as there may be severe hold ups due to insufficient draft depth or barge pilots needing to devise alternative routes to compensate for such problems. Planning and coordination of this kind of logistic exercise become highly knowledge intensive and so KM becomes a crucial issue.

The trade amongst China, India, America, and Europe account for $65 \%$ of the $250 \mathrm{M}$ plus containers moves around the world each year. According to The Economist ${ }^{36}$, with world merchandise trade growing by around 15\% a year and China's exports at nearly twice that rate, the boom in container shipping is set to continue well into the foreseeable future. However, such an apparent constant expansion on the east-west-east trades or better called "round-a-world services, mean that bigger ships are being replaced by even bigger and newer vessels for the above trade lanes, leaving the former vessels to sail northbound and southbound. This is

\footnotetext{
${ }^{35}$ Containerisation International is a well known maritime trade journal.

${ }^{36}$ The Ecnomist ; Conainer Shps: Maxing out.; Vol.382, Nbr.8518; (March 2007); p71.
} 
primarily what led to a serious saturation in of smaller vessels seeking markets in South America as a whole arising of a search of larger economies of scale on the round-a-world services.

There may be little doubt of the role to be played by knowledge creation and sharing on the part of the Corridor members when facing such a challenging scenario. This naturally leads to taking leading steps towards fostering change and innovation based on a rather positive attitude and comprehension of both knowledge generation and sharing as useful corporate mechanisms to face the unpredictable and a definitely mutating business environment.

Global port terminal operators saw a market expansion opportunity in South America in view of the on-going trade liberalization, which later turned into an imperative if they wanted to keep their global standard. Naturally, the mentioned enlargement of vessels' economies of scale becomes associated with a critical need for adequate port handling facilities and by significantly updating existing facilities or purchasing updating on state-of-the art container cranes, transfer and handling equipment, and further facilities. The magnitude of this investment often fell outside the financial capabilities of the regional companies in that market space. A this point, once again, the knowledge creation and sharing became of high relevance at the Corridor roundtables in that those who felt threatened - either directly or not - became aware of their rather fragile condition before the upcoming global trends facing them at that time.

Similarly, global ship-owning companies, global air-cargo companies started to expand their operations too into South America and leading to a reengineering process of the air infrastructure business too. A similar situation happened with other sectors such as barging, trucking, warehousing, freight-forwarding, underwriting, and brokerage. Mapping 'who knows what' in an organization leads to the creation of a knowledge inventory - but does nothing to guarantee the on-going availability of knowledge, and what is even more delicate, nothing guarantees either that when an individual leaves the company, he or she does not take the acquired knowledge quota with him or her. The Corridor has tried to create the necessary forum for helping companies not only create knowledge they would later use, but also sharing same and diversifying its utilization within both the company and the Corridor - as a way to preserve what they have achieved and would potentially accomplish as they develop these knowledge strategies. 
Nonaka and Takeuchi (1995) contend that creating new knowledge is the product of a dynamic interaction among a number of roles. Front line employees deal with the day-to-day issues within the company and therefore find themselves in the best position to experiment what the realities of their business turns out to be - in terms of product or service acceptance, technology, quality, responsiveness etc. These individuals are referred to by Dixon $(2000, \mathrm{p} 11)$ as those who learn from doing the organization's tasks and who therefore possess a kind of knowledge defined as “common knowledge”.

She further argues that the specificity of their knowledge is precisely what gives the knowledge gained from experience its potential to provide an organization with a competitive edge. However, Nonaka and Takeuchi (1995) contend that despite these individuals possessing a considerable amount of highly specific information, they often find it extremely difficult to turn that information into useful knowledge - which seems to lead to both a knowledge creation and sharing problem. This author argues that sometimes the market signals can become so ambiguous and vague that hinders employees from effectively socializing (tacit to tacit) or articulating (tacitto-explicit) knowledge. Other individuals within the front line can become so concentrated or identified on their specific task, that they loose a broader vision of the business environment their firm is engaged in.

Other situation that may occur lies in those who manage to acquire, firm up, or even socialize knowledge, find it difficult to get it accepted in its real dimension or importance on the part of the receiver - who often interprets that knowledge to fit more his/her particular perspective of a given situation with respect to a specific context, instead of adopting a broader view of the situation. Therefore, Nonaka and Takeuchi (1995) argue that there is a continual shift in meaning as new knowledge turns out rather diffuse in an organization. Further, the above illustrates a problem known as the 'stickiness of knowledge' its difficulty in getting transferred. The concept of 'sticky knowledge' has been discussed for a long time and was rigorously studied by Szulanski (1996; 2003). He identified seven sources of knowledge stickiness:

1. Source Lacks Motivation (unwillingness to share knowledge); 
2. Source lacks credibility (the source lacks authority, expertise or is perceived as unreliable or untrustworthy);

3. Recipient lacks motivation (doesn't care);

4. Recipient lacks absorptive capacity (has not the background to perceive cause and effect links, lacks underpinning knowledge or experience in experimentation to know how to use the knowledge);

5. Recipient lacks retentive capacity (forgets vital details);

6. Barren organizational context (the culture or governance structure inhibits knowledge sharing); and

7. Arduous relationship between source and recipient (lack of empathy, trust or commitment to collaborate in the task of sharing knowledge).

He concluded from testing his model (based upon canonical correlation analysis of a data set consisting of 271 observations of 122 best-practice transfers in 8 companies) that contrary to conventional wisdom that blames primarily motivational factors, major barriers to internal knowledge transfer are:

- knowledge-related factors such as the recipient's lack of absorptive capacity;

- causal ambiguity; and

- an arduous relationship between the source and the recipient (Szulanski, 1996).

Despite the above, this may be so, the experience of knowledge creation and its communication or dissemination throughout the network within the Corridor worked in a different way. In this case ambiguity or diffuse knowledge was observed as negligible, possibly based on organizations and people participating in Corridor activities being encouraged and motivated by the seriousness that the threat of globalization posed on almost all players in the region. Therefore, knowledge creation seems to be of paramount importance in the MERCOSUR Atlantic Corridor project as well as knowledge sharing, without which and in spite of accomplishing a remarkable level of knowledge creation, little would have been successfully transmitted to other member companies. This seemed to minimize what Nonaka (1991) and Szulanski (1996) had defined as knowledge ambiguity and vagueness. 
Knowledge has first to be generated to later be transferred to others for which a number of elements have to be considered. When knowledge is intended to be transferred from company to company, or from a team-to-team basis, much depends on the receiving team's absorptive capacity (Cohen and Levinthal, 1990 p128-152). The idea behind this concept is that to learn something new, a company or a team needs already having enough related knowledge to be able to absorb the new knowledge. Research evidence shows that lack of absorptive capacity on the part of the receiving team may represent a significant barrier to knowledge transfer (Szulanski, 1996 pp437-441). Jewell and Walker (2004) argue that a consequence of poor absorptive capacity is often an inability to understand cause and effect loops leading to what Szulanski (2003) defines as causal ambiguity or the condition where people are not able to confidently make a cause an effect link.

Dixon (2000) explains that the similarity of task and context between the source group and the receiving group and the absorptive capacity of the receiving group are deciding factors in determining what kind of transfer method will be most effective. She distinguishes five categories of knowledge transfer:

Serial Transfer: a team that does a task to later repeat it exactly the same way though in a different context. The source team and the receiving team are one and the same. This type of knowledge transfer might be related to the support activities to the various logistic segments where no market variables are assessed nor play a decisive influence, but that form part of the technological back-up that becomes necessary to run operations worldwide (e.g. extranet systems for port terminal operators; global communications for air-cargo freighters; ship-shore interface communication systems for ocean-going vessels and its on-shore facilities; GPS cargo tracking systems for railing, trucking, or distributing companies).

Near Transfer: it involves a source team that transfers knowledge to a receiving team for a similar task and context - though in a different location. It involves the put into practice of explicit knowledge and it therefore aims at generating cost and time savings throughout a period of time. This type of knowledge results easy to transmit as long as it keeps within the very same logistic segment, as technology and operations tend to differ greatly between the different sectors. 
Far Transfer: it involves the transfer of non-routine tacit knowledge between two teams, meaning that the knowledge to be transferred lies primarily in the heads of those who possess it. This is perhaps the most typical knowledge interaction within the Corridor members and the one that brought about a wide spectrum of converging opinions, views, and perspectives - which later on proved useful in triggering the very concept of knowledge socialization on a wide array of issues that were impacting on their business.

Strategic Transfer: this type of knowledge involves a mix of tacit and explicit knowledge that combine with each other to give birth to a new organization in the form of a joint-venture, acquisition, alliance, or the launching of a new service. This type of transfer also played a key role when companies started their search of a strategic partner to face a business environment they have never experienced nor anticipated.

Expert Transfer: it aims at transferring explicit knowledge about very infrequent tasks. This is not a very relevant category as it can be interpreted and put into practice in the form of a procedure or formula. At some point within a VSA ${ }^{37}$ platform it is likely this type of knowledge had played its role in view of the VSA's own specificity and uniqueness in the logistics field.

It became apparent from the author's reflection upon his own experience about the roundtables, discussion with others, and recorded evidence that both explicit and tacit knowledge play an important role at the Corridor roundtables discussions, the latter perhaps being the most critical to both transfer and retain. As difficult as it is to codify tacit knowledge, its substantial value makes it worth the effort.

Davenport and Prusak (2000 p81) argue that mapping 'who knows what' in an organization creates an essential knowledge inventory, but does nothing to guarantee the on-going availability of knowledge. These factors are now illustrated, from my reflection, as being relevant to the

\footnotetext{
${ }^{37}$ VSA means Vessel Sharing Agreement and it refers to the typical association ocean transportation companies got involved with in order to lower their operational costs, increase their load factors, expand market cover, improve their IT systems, and gain overall competitive advantage. Typically a VSA entails a formula that gets adapted to the number of members joining a VSA.
} 
Corridor. Having access to knowledge only when its owner has time to share it or losing it entirely if the "knowledge worker" (Drucker, 1999) leaves the company, is a significant problem that threatens the value of the organization's knowledge capital. This has been a rather new problem members to the Corridor have experienced as they found themselves practically obliged to share their knowledge capital with one another in order to ultimately defend themselves from a threatening business scenario. Furthermore, a much of that tacit knowledge was also vulnerable to be exploited by foreign companies in their search for regional expertise. Consequently, what before was seen with indifference by the regional players in view of a rather indulgent business environment, all of a sudden became a critical factor to manage within their own organizations as well as at the Corridor forums when looking for allies, strategies, and alternative ways to keep in business.

A practice that probably proved to be of paramount importance was the formation of communities of practice (CoPs). Wenger and Snyder (2000) describe a CoP as a group of people who share a concern, a set of problems, or a passion about a topic, and who deepen their knowledge and expertise in this area by interacting on a regular basis. Both companies and individuals get together in CoPs to create tools, standards, generic designs, manuals, potential strategies, and other documents - or they may simply develop a tacit understanding that they share. By doing so, they accumulate knowledge, become informally bound - at least in principle $^{38}$ - by the value they find in learning together.

Wenger et al. (2002 pp6-10) argue that in their view knowledge has become a strategic resource, companies need to keep it on the cutting edge, deploy it, leverage it in operations, and spread it across the organizations by cultivating CoPs in strategic areas. Skyrme (1999 p170) contends that while some communities focus on a particular profession or discipline, the most powerful communities turn out to be customer or problem focused by transcending disciplines and bringing in different perspectives. Naturally, just as a knowledge team is more cohesive than a simple work group, a knowledge CoP results in a more cohesive cluster within a diffuse knowledge

\footnotetext{
${ }^{38}$ The idea behind the Corridor roundtables is to let companies and individuals interact with one another by exposing their problems and exploring alternative potential solutions. A second stage of development means that these players give up their informal bounding condition and enter into joint-ventures, partnerships, alliances, or any sort of association that enable them better cope with the market trends.
} 
network. Skyrme (1999) further agues that the main difference with a work team is that a CoP is self-selecting and cannot be strongly directed but that on the contrary, the best management style for a CoP would be a hands-off approach, whilst providing a climate in which they thrive - an issue that will be extensively dealt with in the coming chapters.

Wenger and Snyder (1998) list the following stages of community development which provides a good match of part for the Corridor development:

1. Latent: there is potential for a CoP within the organization.

2. Coalescent: members come together and recognize their collective potential.

3. Active: engaged in developing a practice.

4. Legitimized: recognized as a valuable entity.

5. Strategic: central to the success of an organization.

6. Transformational: capable of redefining its environment.

7. In Diaspora: dispersed but still alive as a force.

8. Memorable: no longer very relevant, but still remembered as part of member's identities.

The Corridor project appears to have successfully gone through all the stages and finds itself now active with respect to the sixth stage while in permanent search of reinforcing all and every of the precedent stages in a sort of knowledge-based recycling system. Good communication and shared sense of purpose, intensive external networking, effective knowledge management and trust make up the guiding principles of the Corridor and proved useful in setting up a knowledge market ${ }^{39}$.

Davenport and Prusak (2000) contend that their studies show that three factors in particular often cause knowledge markets to operate inefficiently in organizations: the incompleteness of information about the knowledge market; the asymmetry of knowledge; and the localness of knowledge. These factors are now illustrated, from my reflection, as being relevant to the Corridor.

\footnotetext{
${ }^{39}$ A knowledge market is a place where knowledge buyers (those in search of knowledge) and knowledge sellers (those who possess knowledge) get together to exchange knowledge and firm-up interactions and relationships.
} 
If the MERCOSUR Corridor is to be considered as an organization made up of a great number of sub-organizations, it can then be argued that the incompleteness of information occurs when firms do not know where to find their own existing knowledge. The lack of knowledge maps and "yellow pages" ${ }^{40}$ that link buyers and sellers is a typical problem for which the formation of CoPs turn out to be useful. Typically, a common lack of knowledge that was found to exists was the absence of thorough explicit knowledge on both how to assess and what is the operating costs and competitive margins for medium-sized ocean and river transportation companies is, whereas this knowledge turned out to be possibly one of the strongest knowledge assets that global companies brought into the region.

Asymmetry of knowledge occurs when there is abundant knowledge on a subject in one location and a shortage of it somewhere else within the same organization or group. This situation brought about a fertile ground to formulate and put into practice a solution to this problem through the creation of a Corridor-like forum where knowledge asymmetries found the ideal forum to help knowledge sellers and buyers meet and offset these asymmetries. These asymmetries became apparent not only between complementary segments within the logistic field, but also within the same area of activity (e.g. railways; coastal feeding services; inland depots; ground transportation firms; freight-forwarding companies; etc).

Localness of knowledge also represents one of the pillars upon which the Corridor concept along with its many CoPs seems to work well in the direction of knowledge enhancement. The knowledge market generally depends on trust and as such, people and organizations tend to trust those who they know. Face-to-face meetings - a subject to be dealt with in a later chapter - are often regarded as the best way to let tacit knowledge flow and disseminate through an organization or a CoP. CoPs are not only competing for market share, but provide a fundamental tool to find, nurture, and retain talent - all those concepts being of paramount importance for the great majority of the Corridor members to face uncertainty within a rapid and ever evolving business scenario.

\footnotetext{
${ }^{40}$ Often called as on-line directory of expertise in an organization where knowledge comes structured by skill and discipline and not so much by department. It often entails best practices databases and expert profiling.
} 
Following from the 'localness of knowledge' referred to above, it is useful to briefly discuss how the concept and theory of situated learning (SLT) comes into play with the development of CoPs. Lave and Wenger (1991) in their book discuss how learning in communities has evolved from a kind of apprenticeship model. Wenger then as noted earlier advanced the theory to explore the realities and characteristics of CoPs. Sense (2008, p37) states that "learning is always considered a practical accomplishment that takes place amongst and through other people (where learners construct their meanings and understandings and learn through their social interactions within a context) and is not simply and only, an individual cognitive activity (where learners as individual actors possess and process information and modify their mental models)”. He argues that SLT is a highly social process where the context and situation resides within the practice being undertaken, where there is an exchange of knowledge that frames and re-frames meaning, and it becomes a natural part of working action as learning. Also he argues that SLT supports the notion that learning is more that cerebral activity but is commonplace and practical but does require reflection, discussion and collaborative sensemaking. This can be contrasted to formal 'classroom' learning or other forms of knowledge transfer where the context is not heavily embedded. SLT also requires institutional support, such as that of the Corridor and participant organizations that actively engage in co-learning between members through round table discussions and joint venture business formations. The key aspect of SLT that relates specifically to the Corridor is that the context must be favorable to participants working out the best way they can learn from each other. This requires an intervening entity that helps participants match their learning and cognitive styles (such as the Corridor) or that participants self-organize to ensure that their interactions provide meaningful learning through work interactions. In this way SLT supports the idea that the Corridor could be viewed as a form of CoP.

\section{8 - CULTURE RELATED TO THE CORRIDOR PROJECT}

Culture has been described by Schein (1993) as displaying itself at three levels. Artifacts are observable at a surface level through such things as flags and historical accounts. At a deeper level we may perceive espoused values through acceptable ways of doing things in that certain actions and approaches are approved of and openly encouraged while others are frowned upon or receive explicit criticism. At the deepest level lie basic assumptions that are inferred and understood by those sharing that culture - these are the ways of doing things that are implicitly 
followed. This view of culture helps explain how we can understand surface artifacts and actions in terms of deeper cultural drivers.

The Corridor project embodies a number of neighboring South American countries whose cultural roots might well be regarded as of similar nature. However, there is sufficient evidence behavior, observed and encountered, that suggests cultural gaps existing not only between the MERCOSUR countries but even within single nations such as Brazil. Thus even within some of these countries, culturally they may be considered as various countries inside a single country.

Without aiming at breaking down the social components roots of each of the MERCOSUR members, it is useful to assess and compare the national cultures of the two largest economies Brazil and Argentina - by following the pioneering working model of Hofstede (1980) with respect to the four dimensions of culture-related values. This comparison is of general value in understanding cultural impact upon Corridor member interactions. While the Atlantic Corridor's aims are focused upon joining countries both of somewhat similar cultures such as Brazil and Argentina it also has longer term goals to include in the MERCOSUR, distant cultures such as South Africa. An additional cultural influence is that countries that belong to the region are also greatly influenced by their indigenous communities such as is the case in Venezuela and Peru and further to Mexico. The following findings on cultural characteristics reported by Hofstede (1980) illustrates some of the influences at play. Caution is needed when trying to interpret Hofstede's findings because his data was based upon a study within IBM several decades ago and both times and globalization forces over past several decades have probably changed some cultural characteristics. This section in the thesis is meant to support understanding, in general terms, the cultural environment present in the Corridor project.

Power distance (PD) : is the extent to which inequality is seen as an irreducible fact of life or the extent to which employees accept that their boss has more power than they have and that their boss's opinions and decisions are right because he or she is the boss (Hoecklin, 1995: Chapter 2). While Brazil ranks $14^{\text {th }}$ (PD score 69), Argentina is $35^{\text {th }}$ (PD score 49). Brazilians share their PD area with West Africa, Singapore, Hong Kong and Colombia, whereas Argentines do so with Italy, South Africa, Jamaica. Even though this information is insufficient to draw any firm 
conclusions, it suggests that while Brazilians are more inclined to obey and honor what the boss has to say - probably influenced by a more authoritarian immigration flow from Portugal and West Africa, Argentines look not so much aligned with the boss' directives but a bit more open to challenge and discuss - probably because of the country's European immigration flows. In other words (and this is reflected in my own experience) Brazilian managers can be seen as making decisions autocratically and paternalistically; exerting close supervision positively evaluated by subordinates; centralizing decision-making; supporting top-down pyramidal organizations; and accepting autocracy at whatever level within the organization. Argentine managers, however, appear more identified with less centralization; flatter organization pyramids; sharing decisionmaking to a certain extent; close supervision negatively evaluated by subordinates; and autocracy is better valued at lower organizational layers. These differences loom large in the northern and less industrialized part of Brazil whose culture has not been yet influenced by the on-going globalization trends and where tradition plays a fundamental role. Understanding and evaluating these issues appropriately turns out of immense value at the time of negotiating a joint-venture, alliance or whatever commercial and industrial undertaking at regional basis as well as when exchanging and sharing knowledge and business intelligence.

- Uncertainty avoidance (UA): is the lack of tolerance for ambiguity and the need for formal rules or the extent to which a people feel threatened and try to avoid ambiguous situations. While Argentina ranks $15^{\text {th }}$ (UA score 86) - together with Chile / Spain / Costa Rica / Panama - Brazil ranks $22^{\text {nd }}$ (UA score 76) - together with Venezuela and Italy. High UA means more worry about the future; more emotional resistance to change; tendency to stay with same employer; loyalty to employer is seen as a virtue; less risktaking; conflict in organizations is undesirable; initiative of subordinates should be kept under control; rules should not be broken. Both countries show very high UA ratios though they do not match Hofstede’s definitions - which if strictly applied to and considered with respect to the Corridor cannot find much correlation with the exception of more worry about the future and more resistance to change. At this point it turns out interesting to highlight that Argentina shows a higher UA ratio than Brazil which can be explained by the fact that the former nation has shown throughout its history more political and economic crises than Brazil leading to a general feeling of more fear and 
resistance to change. However, and taking into account both countries as a whole within the context of the Corridor project, the above suggests that despite holding a very high UA level, it was the ever-evolving business and political environment that made the whole system work through combining efforts, innovating and eventually gaining competitive advantage. No joint-venture, alliance, partnership or cooperation agreement of any sort would have been otherwise feasible without having both managers and employees overcoming their fears and differences while participating in the roundtable forums across the region.

- Individualism (IDV): is a concern for oneself as an individual as opposed to concern for the priorities and rules of the group to which one belongs. Argentina ranks $23^{\text {rd }}$ (IDV score 46) along with India and Japan, whereas Brazil ranks $27^{\text {th }}$ (IDV score 38 ) together with Arab countries, Uruguay and Greece . A low IDV score - as shown by both countries - suggests that: employees expect organizations to look after them like family and also to defend their interests; employees believe in group decisions and hold a solid belongingness perception; less concern in fashion with management ideas; involvement of individuals with organizations primary moral; and that promotion is based on seniority rather than on merit. Conversely, a high IDV score suggests a much higher degree of individual decisions and initiative or achievement, further to giving much more value to modern management concepts and promotion based on merit. The Corridor, however, is not an individual but an organization that embodies a growing number of different firms and corporations and as such seems to gather some characteristics of a low IDV score and some of a higher IDV score. Again, suggests that it is the business environment into which an organization is immersed that dictates any necessity to shed old paradigms and quickly adjust to new realities in order to survive. Seniority is no longer a factor in South America but merit is all; belongingness is nowadays in decay and individual initiative and even entrepreneurship is more and more valued and respected by both society and peers; paternalistic leadership is no longer sought or expected in the way it probably was in the past; modern management techniques are often assessed and adjusted to the regional needs. All these elements that in fact constitute a strong managerial trend, suggest once more it is the business context the one making culture subordinate to 
achieving goals and objectives, and not the opposite. When survival is at stake, all the cultural variables seem become adapted to accomplishing the desired objectives.

- Masculinity (MAS): masculine societies define gender roles more rigidly than feminine societies. Argentina ranks $21^{\text {st }}$ (MAS score 56) along with India, Hong Kong and Greece, whereas Brazil ranks $27^{\text {th }}$ (MAS 49) together with Pakistan, Singapore and Israel. A high MAS level (Argentina) presupposes that some occupations are restricted to male; that organizational interests are a legitimate reason for interfering with people's private lives; that fewer women are available in more qualified and betterpaid jobs; that male are better qualified to deal with stressing jobs and industrial conflict. A lower MAS level (Brazil) means practically the opposite. The Corridor is mostly integrated by male managers and employees. However and according to the author's personal experience at the forums, this is due more to the fact that logistics in whatever segment is considered a male activity, with little or no room for females. Appointing a woman as a port director, a railway manager, an air-station operations manager, a shipping manager, or a warehouse sales executive does not have anything to do with her real possibilities of succeeding at the job, but with the way she will be perceived by others in a typically male environment. In this case, productivity is to suffer as well as the whole system. Needless to say that both Argentina and Brazil rely upon females in their respective armed forces where cultural barriers seem to have adapted or been subordinated to the political will. With the exception of the armed forces - which is a top-down decision and therefore assimilated as such - the above suggests being a matter of how societies perceive the working roles according to their cultural roots. In this regard Lucey (1997, p22) argues that the process of perception is individual and varies from time to time and that people attach meaning to messages and situations in accordance with their attitudes, experiences and value systems. In other words, people tend to see and understand what they want and expect to see and understand, which is undoubtedly influenced by culture and pre-concepts. 
A more recent and more extensive international cultural study was undertaken in at the end of the 1990's and was reported upon in 2002 (House, Javidan, Hanges and Dorfman, 2002) and is referred to as the Global Leadership and Organizational Effectiveness (GLOBE) study. This study supported some of Hofstede’s previous seminal work (Hofstede, 1980) but identified 9 dimensions. The above were included and others offered. The dimensions are (House et al., 2002, p5-6):

1. Uncertainty avoidance (as described above);

2. Power distance (the degree to which members of an organization or society expect and agree that power should be equally shared);

3. Collectivism I - Societal collectivism (that reflects the degree to which organizational and societal institutional practices encourage and reward collective distribution of resources and collective action);

4. Collectivism II - In-Group Collectivism (that reflects the degree to which individuals express pride, loyalty and cohesiveness in their organizations or families);

5. Gender Egalitarianism (similar to the masculinity dimension);

6. Assertiveness (the degree to which individuals in organizations or societies are assertive, confrontational, and aggressive in social relationships);

7. Future orientation (the degree to which individuals in organizations or society engage in future-oriented behaviors such as planning, investing in the future, and delaying gratification);

8. Performance orientation (the extent to which individuals in organizations or society encourages and rewards group members for performance improvement and excellence); and

9. Humane orientation (the degree to which individuals in organizations or society encourages and rewards individuals for being fair, altruistic, friendly, generous, caring and kind to others.

The GLOBE study did not have any data from Latin America but included the Latin Europe cluster that includes Spain and Portugal as well as Italy. These three cultures, through mass immigration, influenced Latin American culture. The GLOBE study measures not only 
perceptions from a well designed survey instrument, for details refer to (House et al., 2002), of how things are but also perceptions of how things should be. This gives a richer and fuller picture of the cultural norms. The Latin Europe cluster results are presented in Figure 3-4 (Jesuino, 2002, p85).

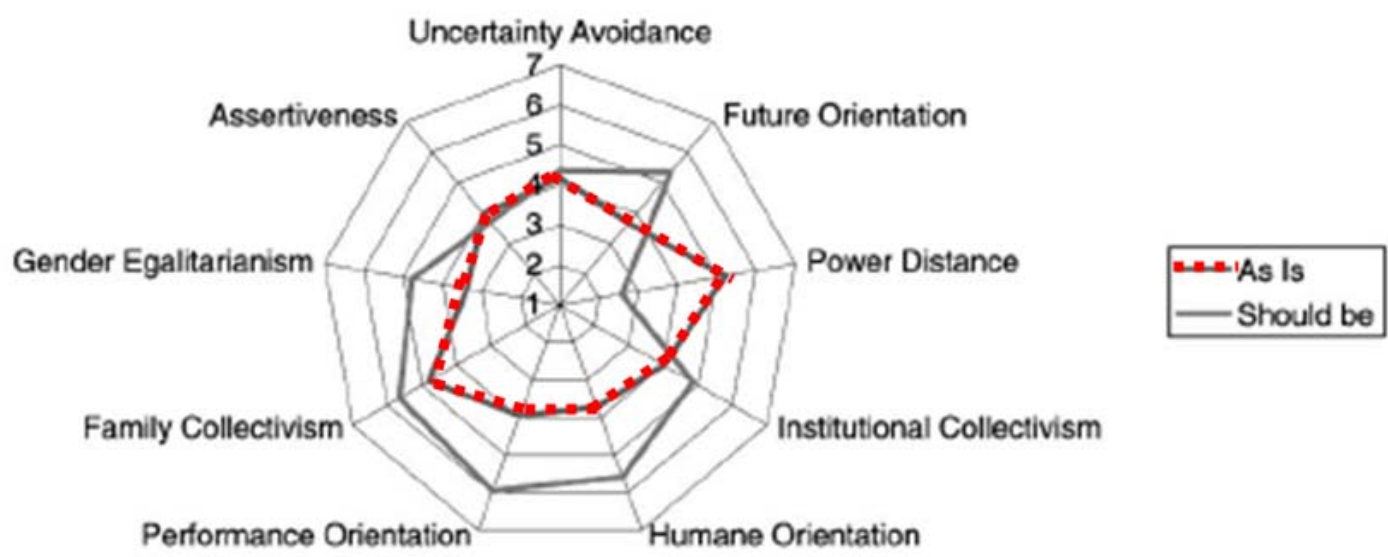

Figure 3-4: Cultural Map of Latin Europe Cluster of Countries

Again this provides supporting evidence that helps us better understand the cultural drivers of behaviors in general terms. The implications of this relates to the way that Corridor members interact in sharing knowledge, in negotiating agreements and in the way that 'chemistry' of attraction or lack thereof may be explained and understood in cultural terms.

\section{9 - PROJECT MANAGEMENT CONCEPTS APPLIED TO THE CORRIDOR PROJECT}

As stated earlier, the Corridor can be seen as not just a very large project but rather a program of projects. Moreover, it has been proposed earlier that the Corridor may be seen as both a form of $\mathrm{CoP}$ as well as having some attributes shared by a project management office. Therefore a discussion on the nature of a project, PM and PM maturity helps to provide a framework for testing how the Corridor functions. 
The $\mathrm{PMBOK}^{41}$ guide $^{\mathrm{TM}}$ defines a project as a temporary endeavor undertaken to create a unique product, service, or result, whereas it refers to project management as the tasks that are carried out in an environment that often influences the organization more broadly than the project itself. Project management is also described as "the application of knowledge, skills, tools, and techniques to project activities to meet project requirements” (PMBOK, 2004).

From a process point of view, the PMBOK defines project management as the application of knowledge, skills, tools and techniques that receive inputs and generate outputs. The KM perspective of project management processes is further deepened by addressing the project integration management knowledge area that includes the processes and activities needed to coordinate the various existing processes within a project. The PMBOK also refers to project scope management as the processes required to ensure that all the work related to the project completion is included by both identifying and controlling what effectively is needed within the project. Along with it, project risk management concepts get naturally linked to project completion in the form of increasing the probability and impact of positive events, as well as decreasing the probability and impact of events that turn out adverse to the project.

A further PM concept that is needed to be explained in this thesis that helps explain the nature of the Corridor is the viewing the Corridor as a form of project management office. The project management office (PMO) concept has been with us for a long time. Walker, Arlt, and Norrie (2008a) state that most construction projects tend to be managed on site through a project office. This office is where specialists and much of the project team congregate in physical proximity to work together, to share ideas and to facilitate communication through rich personal contact. More recently however, the PMO concept has been evolving as a way to move beyond just gathering people together and better coordinating their actions and resources towards sharing standards (Rad, 2001; Ingebretsen, 2003; Blomquist and Müller, 2006; Crawford, 2006). This facilitates program or portfolio management (Turner, 1999: chapter 14; Artto, 2001) in a way that adds coherence. Program and portfolio management bears distinct similarities with the way that the Corridor operated.

\footnotetext{
${ }^{41}$ PMBOK: Project Management Body of Knowledge
} 
Walker, et al (2008a) also state that a PMO at its lowest level provides a location where project team members gather to do their PM work and this is often restricted to those involved in the 'iron triangle’ processes of time, cost and quality control. This level of PMO supports decision making, coordination and control and is a way of 'housing' project managers. The number of functions performed are quite varied but generally with governance and training functions and as Hobbs and Aubry (2007) report, most have been formed in the last 2 years and about $80 \%$ being formed within the last 5 years. Hobbs and Aubry (2007, p82) also report that the functions of a PMO tends to be to:

1. Develop and implement a standard methodology;

2. Promote project management within the organization;

3. Develop competency of personnel including training;

4. Provide mentoring for project managers;

5. Provide a set of tools without an effort to standardize.

They found, however, that for $58 \%$ of the sample of 500 usable survey responses, the relevance or even the existence of the PMO had been seriously questioned (Hobbs and Aubry, 2007, p83). Handler and Magee (1999, p2) through the Gartner group suggest three classifications of PMOs in terms of information systems implementation for health care organizations (HCOs). These are:

1. “The Repository Model: The project office serves as a source of information on project methodology and standards;

2. The Repository-Coach Model: An extension of the repository, this organizational structure assumes a willingness to share some project management practices across business functions and uses the project office to coordinate communication. Best practices are documented and shared, and project performance is actively monitored. Results are used as an opportunity to raise HCO performance and to train inefficient or new project managers. In some organizations, mentoring relationships have been established across business boundaries between high performing project managers and those who are less able. The project office in this model is a permanent structure with staff and has some supervisory responsibility for all projects; and

3. The Repository-Coach-Manager Model: The most permanent, consolidated organizational model, this concentrates project management within the project office. This implies direct 
management or oversight, depending on scope and duration, of projects wherever they occur within the HCO. In some cases, all project managers are actually staffed within the shared service and consigned to projects as needed. This model assumes a governance process that involves the project office in all projects regardless of size, allowing it to assess scope, allocate resources and verify time, budget, risk and impact assumptions before the project is undertaken. Funding is generally a combination of direct, budgeted allocation for baseline services and a fee-for-service charge for others.”

Another example of a PMO that won internal awards within the communications organisation Ericsson $\mathrm{P} / \mathrm{L}$ that was based in Melbourne, Australia is described and analysed by Cartwright (2006; Cartwright and Walker, 2008). This PMO started life, as does many others, as an attempt to raise PM skills and capabilities. Its original focus was concerned with creating standards, promoting learning exchange and sharing experience and gathering data on success or otherwise of their projects. This evolved to being involved in strategic decision making about which projects to focus on but also it was involved in helping groups within the organisation develop business cases. In addition it acted as a coach for PM staff as well as advised sponsors and champions. The PMO in its latest evolution is proactive and has been made more accountable (Cartwright and Walker, 2008).

Once PMOs provide a physical or communities of practice (CoP) link to project management teams then knowledge sharing takes place and this allows standards to be set that make established PM processes more repeatable for that organization. This level of PMO moves to a higher order PMO that is described by Christenson (2007) as a centre of excellence and this appears to be a very effective way of transferring knowledge (Walker and Christenson, 2005). Thus the PMO facilitates organization-wide impact of an incremental improvement and increased PM maturity.

The PMI standard OPM3 (PMI, 2003) specifies a 5 level maturity model:

1. Initial, where ad hoc PM approaches are deployed and the project is characterized by chaos and confusion with the same mistakes being repeated for each project; 
2. Repeatable, where basic PM tools, techniques and processes are deployed and formalized often with a start in developing a procedures manual or system that allows success to be repeatable or failures not repeated;

3. Defined, the procedures manual or system is formalized and tested to a point where it is accepted as a policy and guides PM activities;

4. Managed; at this stage performance is recorded and data collected to demonstrate the effectiveness of the systems in place in the PMO and it is possible to justify resources and initiatives and gauge the value of the PMO and the systems are well understood and accepted; and finally the

5. Optimizing level may be reached, where it is continuously improved and is embedded in the organizational culture (Crawford, 2002; Rad and Levin, 2002, p282).

The proposition that arises to be later tested from this discussion of PMOs, COEs and CoPs is that the Corridor has functioned in part as a high level transnational cross-organizational repository-coach type $\mathrm{COE}$ through its $\mathrm{CoP}$ activities in addition to its mentoring, supporting standards and knowledge transfer. Its maturity level could be said to be at level 4 and possibly, at least in part, at level 5.

All these PM concepts find a number of links within the Corridor project that turn out essential to consider it from a project management perspective and can be summarized as follows:

I. The concept of temporariness means that every project or program of projects has a definite beginning and a definite end - at least in principle. Normally, the end of a project comes when its objectives have been accomplished or when it becomes apparent that the objectives cannot be achieved or for whatever reason the project is abandoned. The MERCOSUR Atlantic Corridor project is a sort of master project that serves as a framework and strategic guidance for a growing number of sub-projects that constitute and give strength to the Corridor. These subprojects are the ones showing a definite beginning and a concrete end with respect to their temporariness, whereas the master project or Corridor project has been undertaken to create a lasting end - to be a permanent forum of knowledge interaction aiming at creating the necessary tools for regional companies to face the impact of globalization. These tools become associated with a number of possibilities that range from a simple reengineering strategy after having 
acquired the needed expertise, to joint-ventures, mergers and acquisitions. While each sub-project usually has a limited time-frame associated with its own particular opportunity or market window, the Corridor project pursues economic, social, and operational impacts that far outlast the sub-projects themselves. The very dynamic and long-lasting nature of the globalization process makes the Corridor project's goals ${ }^{42}$ lie distant over the horizon, while it brings about a growing number of business situations that turn into sub-projects with definite start-ups and ends.

II. The concept of uniqueness is an important characteristic of project deliverables. Contrary to what happens in many industrial projects around the world, the Corridor project appears to be truly unique and not likely to be imitated anywhere at present as it is based on both a world trend - globalization impact - and a regional phenomenon - end of protectionism. The Corridor project was born as a consequence of a very unique world trend that produced a defensive reaction amongst a number of regional companies that were operating in a rather protected and bilateral economic system, and thus gave birth to a number of sub-projects that proved to be unique too. Such a unique characteristic becomes apparent in the way regional firms accepted to get together and naturally exchange what before was probably regarded as strategic information. The way knowledge is generated and transferred makes the Corridor project and its sub-project of very unique nature.

III. Progressive elaboration: from the view that the globalization phenomenon is an evermutating process showing many different and ever-evolving faces and impacts on the region. The Corridor both adapts itself to face the new realities and constantly expands its functions into new roles to better cover the knowledge interaction. The Corridor as a framework or forum provides a number of sub-projects to grow and be shaped, it has its own organizational structure that counts on a progressive description of tasks and duties which starts with the project scope and it develops progressively in a much more explicit detail as the many sub-projects demand it. A significant number of companies formed part of the Corridor project and therefore attend the integration roundtable. These are the

\footnotetext{
${ }^{42}$ The Corridor's main goals can be identified as creating the necessary conditions for companies to let them achieve larger economies of scale and gain competitive advantage. The economies of scale are associated with lowering their operational costs that would ultimately let them gain competitive advantage as to face the global firms. The concept of competitive advantage though depending on a number of variables, is to a great extent linked to the enlargement of economies of scale.
} 
places where the concept of progressive elaboration varies as it is required by a specific sub-project in any of its possible segments.

IV. Project Management Office (PMO): it is the Corridor itself that acts as an organizational unit or PMO that centralizes and facilitates the initiation of projects under its domain. Unlike many organizations where projects are grouped and conducted by a given PMO which is in charge of managing and controlling them, the Corridor recruits, informs, trains, and take all the necessary steps to facilitate companies and individuals generate and transfer knowledge that may help them overcome their ongoing problems. It does not manage or monitor any sub-project but that facilitates the sub-projects to accomplish their goals while adopting the structure of a project itself - which reinforces its uniqueness and temporariness characteristics.

V. Project life cycle: the Corridor project does not match the traditional concept of life cycle in that it is the phases linking the beginning of a project to its end. The typical transition from one phase to another within a project's life cycle in the form of deliverables, does not really happen at the Corridor, but in the sub-projects the Corridor embraces. The Corridor project finds itself in some sort of ever-evolving intermediate phase that creates new deliverables by means of facilitating a constant and growing explicit and tacit knowledge interaction amongst its members. Such an interaction produces a continuous flow of knowledge that keeps the Corridor's life cycle at an intermediate level of development, whereas it makes many sub-projects overlap their stages and adopt a fast tracking schedule as they witness others' successful experiences to put them into practice at once, always under the Corridor umbrella.

VI. Stakeholder Management: Naturally the Corridor project, like any project, engages a great number of stakeholders who directly or indirectly, may have an impact on the project's life cycle and therefore its effectiveness and maturation stage (Walker et al., 2008b). As a transnational project, the Corridor involves very large and influential corporations and entire governmental interests that may play as either positive or negative stakeholders. Large global corporations encountering resistance to their market expansion strategies on the part of the regional firms may be regarded as negative stakeholders by the Corridor members. On the contrary, state and federal governments that try to maintain and expand their local businesses and stopping the migration of assets towards extra-regional 
interests, will probably be regarded as positive stakeholders by the Corridor members. Typically, the Corridor counts on a great and growing number of primary and secondary stakeholders who can make a sub-project slow down or achieve its objectives faster. VII .Project Management Processes: the Corridor project can be defined as a longrun process that integrates a number of sub-projects displaying well defined subprocesses during their life cycles. The purpose of a project is to initiate, plan, execute, monitor, control, and close a project. This is the way the Corridor's sub-projects have been managed throughout the years. However, the Corridor gets differentiated from its sub-projects in that the processes it utilizes are in permanent evolution and put into practice at the integration roundtables across the region. Both explicit and tacit knowledge creation and transfer need a permanent refinement of processes as new situations arise, more innovative solutions are sought, and more sophisticated stakeholders participate.

VIII. Project Integration Management: integration includes aspects such as unification, consolidation, articulation, and integrative actions that turn out crucial to stakeholder or member requirements, to managing uncertainty and expectations, to fostering innovation and knowledge sharing, and to sub-project completion. The integration forums or roundtables represent the physical settings where the processes related to the ongoing sub-projects merge into the Corridor's processes and lead to a flow of knowledge creation and transfer.

IX. Project Scope Management: the Corridor project relies upon a well defined scope of action where a number of functions are defined, are verified and effectively controlled over a specified period of time aiming at creating the necessary conditions for the members to interact and bring about a number of sub-projects. The Corridor's scope defines the way an integration roundtable should work, whether its location is more strategic than tactic, the necessary number and right location, the number of commissions each roundtable should incorporate, and the way they should be moderated and monitored to let knowledge emerge and spread all over the system. The Corridor project scope management therefore includes a number of processes that prove essential for the system to allow sub-projects proliferate across the region and produce more innovative and solid deliverables for their members and itself. 
$X$. Project management tools and techniques find fertile ground and a significant variety of situations where these can be utilized and tested. Both within the Corridor project master project and the existing and ever-evolving sub-projects, it becomes apparent that from a project management perspective, the Corridor and the sub-projects it embraces seem to be making full use of a number of variables that turn out to be key for the project management practice: time; budget; processes; knowledge; temporality; uniqueness; and possibly a number of other factors this dissertation does not intend to focus on. Consequently, this project seems to be an adequate forum where project management tools and techniques can be tested, as well as this project seems to find in them a set of solid tools enabling it conduct its functions in a solid and proactive manner.

\subsection{0- CHAPTER SUMMARY}

This chapter illustrated how both reflection upon practice as illustrated in Chapter 1 Figure 1-2 together with literature used in the DPM coursework component influenced the choice of theory that was then presented to underpin the empirical stage of this thesis

Project management is about implementing a strategy. Therefore, a theoretical exploration of various schools of strategy was introduced in this chapter because of it relevance to understanding the nature of the corridor and how that was shaped by the industrial structure. The Corridor is presented as a response to the contextual forces that Corridor members faced and help explain its reason for being as well as the way it operates. Competitive advantage and the value chain and its relevance to the Corridor expand this discussion. The theory of the firm theory grounded in a resource based view helped to frame a competencies and dynamic capability perspective on how the Corridor evolved and how it operates so that the motivations of the Corridor's participants and its formation can be more clearly understood.

An important part of understanding the Corridor is how it is led and how leadership directs the Corridor's strategies and activities and so a section on relevant leadership theory was included in this chapter. This naturally led to a discussion of business cooperation theory as this lies at the core of the Corridor's reason for being. 
One of the stated prime motivations of Corridor participants and lies at the heart of its existence is knowledge sharing and so it was necessary to review $\mathrm{KM}$ in this chapter and to discuss in particular issues related to knowledge transfer and the formation of CoPs. More critically, as this is a dissertation based on a PM perspective it was necessary to provide a section in this chapter to justify how the PM view can be substantiated as being valid.

In summary, the MERCOSUR Atlantic Corridor can be regarded as a master project with an indefinite scope of time that gives birth and nurtures an apparently ever growing number of subprojects. These subprojects and opposite their master project - the Corridor - show a dynamic life-cycle whose maturation levels depend on the business environment they have to face.

As such, the Corridor project along with its sub-project, all reflect a number of theories which, either directly or indirectly, take part at different levels of the master project while holding a direct influence on the sub-projects. The Corridor project, being of a transnational nature and mostly focused on a knowledge theory dimension, gathers in itself and its sub-projects a rich variety of situations which makes it unique, multiple-theory oriented, and of a rather high complexity in view of its great number of links and interactions. As such, it involves aspects related to competitive strategy where Porter's five competitive forces, industry structure, and generic strategies have first to be looked into and broken down, to later understand how the mentioned subprojects emerged and developed.

Understanding the way the value chain works to actually add value in a business transformation to integrate very different sub-projects by combining their primary and support activities with one another has also been argued to be a significant way to add value to the organization. Further, the theory of the firm helps understanding the motivation behind the creation of the Corridor project, by both companies and individuals before a hostile business environment.

The leadership and strategy theories turn out useful to visualize how the different strategies and leadership styles can best match the distinct situation that evolve as the globalization moves forward. The business cooperation theory helps understanding the steps that many firms take prior committing to more formal types of alliance, further to what their motivations or reasons 
may turn out to be. Finally, both the knowledge theory and the project management tools and techniques applied to the Corridor project result of paramount importance to visualize the direction of this dissertation.

This dissertation aims at contributing to deepen different aspects of the above theories by addressing a project which, in view of its uniqueness and complexity, embraces a great number of variables which far from remaining stagnant, evolve or mutate as the globalization trend expands all over the region. Special emphasis is set on the knowledge dimension and its practical application in full accordance to the generally accepted project management best practices, tools and techniques. 


\section{THE CORRIDOR PROJECT: WORKING THROUGH COLLABORATION AND KNOWLEDGE TRANSFER}

\section{1 - INTRODUCTION}

The purpose of this chapter is to expand upon the broad context of the Corridor described in Chapter 2 and the literature review presented in Chapter 3 to now describe the Corridor in terms of its role as a program/portfolio tool that enables projects to be realized through facilitating collaboration and knowledge sharing.

This chapter commences exploring how knowledge exchange occurred with the Corridor and the relationship between the face-to-face interaction concept and the utilization of technology to ensure a better knowledge sharing and transfer in the roundtable forums. The size of the firm along with some culture-related aspects are also dealt with in an attempt to establish the real influence a technological platform may have on an organization's capacity to transfer knowledge effectively. The emotional filters that may act as barriers in the transfer of both explicit and tacit knowledge between individuals and companies are also linked with the freedom to interact and the face-to-face concept.

The section continues by explaining the existing relationship between a firm's absorptive capacity and the business context it operates through a given CoP. Section 3 follows, providing different examples of knowledge transfer through strategy formulation as a way to establish its real importance as far as vertical integration processes are concerned. Section 4 then explores how the role of a company's home base, in achieving global goals and gaining competitive advantage, also acts as a potential triggering factor for regional cooperation and collaboration.

The chapter ends with the conclusions and recommendations. 


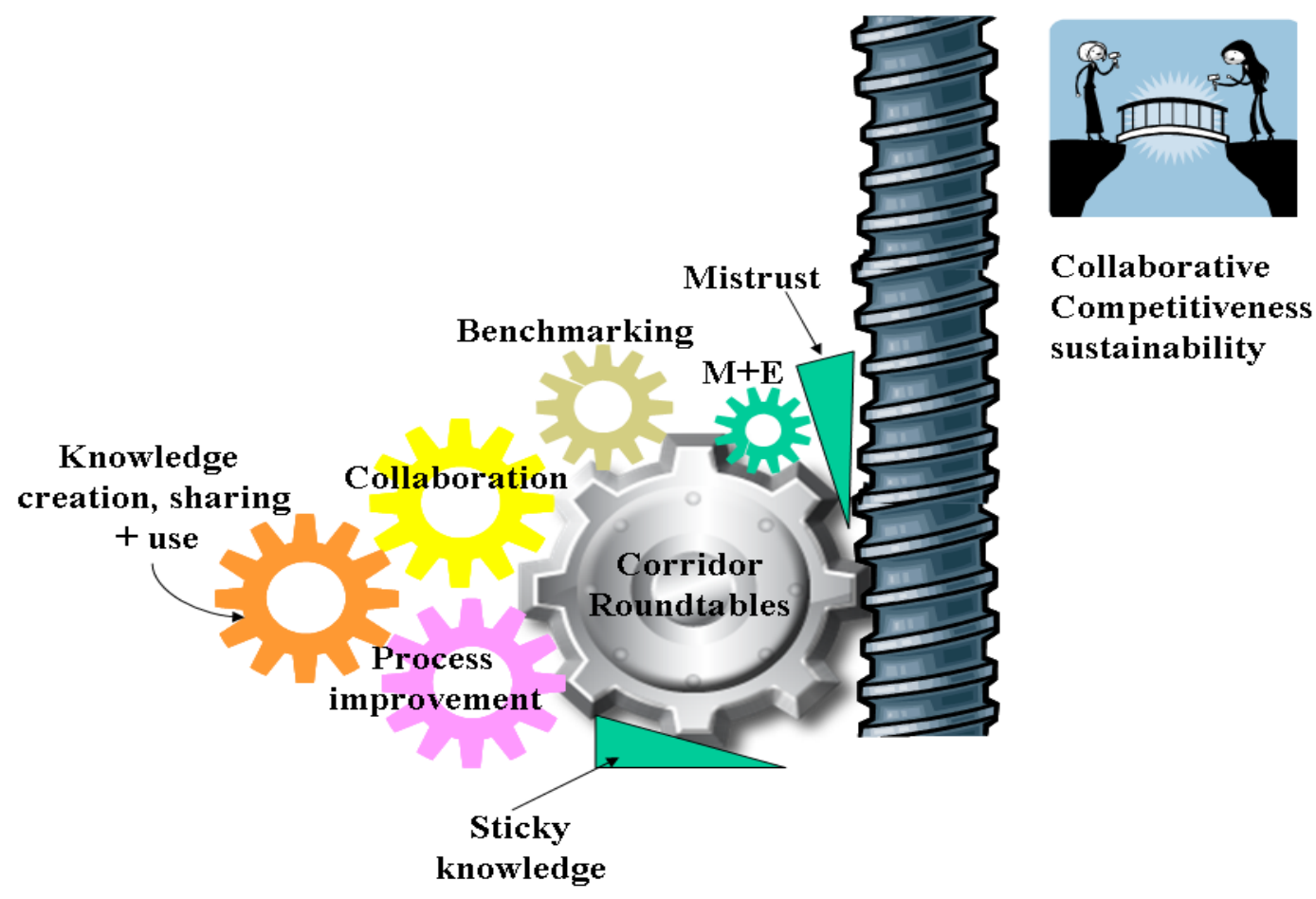

Figure 4-1: The Corridor as a tool for collaborative sustainability

Figure 4.1 illustrates the Corridor and its associated roundtables as tool to facilitate collaboration and through that, global competitiveness and a sustainable business future. The metaphor used is a machine tool that uses synergies of benchmarking, process improvement, forming collaborative groups, enacting strategies that may lead to mergers and acquisitions between members in forwards, backwards and vertical integration of value chains to turn the screw cranking up sustainable competitiveness. This is achieved and through the Corridor facilitation of trustbuilding and knowledge creation, sharing and use. The identified inhibitors are stickiness of knowledge in being effectively transferred and the wedge of mistrust where this occurs to undermine the 'machine tool' working effectively.

\section{2 - THE CORRIDOR AS A KNOWLEDGE TRANSFER DEVICE}

It can be argued that knowledge sharing entails making the existing knowledge of a company more generally accessible as a way to face upcoming challenges and thus gain competitive advantage. The knowledge advantage (K-Adv) concept as proposed by Walker, Wilson and Srikanathan (2004) and set out in more detail by Walker (2004, p6) states that the K-Adv is "the 
capacity to liberate latent creativity and innovation potential through effective management of knowledge both from within its organizational boundaries and its external environment”.

Walker (2004) argues that the K-Adv concept entails a connection between knowledge use and sense-making to build the necessary social capital in the form of creative chaos and ambiguity; redundancy of resources to allow people to think and reflect; variety of stimuli and channels of communication and capacity for reflection and curiosity. This is supported and enabled by a technology infrastructure, a human capital infrastructure, and a leadership infrastructure that together in unison provide the necessary conditions for effective knowledge creation, sharing and transfer. The leadership element needs to be transformational as discussed in Chapter 3 Section 3.5 and it also needs to be strategic in envisioning how a K-Adv environment can be created and maintained.

The human capital element relates to reducing knowledge stickiness, building and maintaining trust that enables and facilitates knowledge creation and transfer. This is also linked to raising the organization's absorptive capacity (Cohen and Levinthal, 1990) and reducing causal ambiguity described in Chapter 3 Section 3.7 as being key elements of knowledge stickiness (Szulanski, 1996). The third element is information communication technology (ICT) and its effective diffusion and adaptation. The way that the Corridor addressed each of these K-Adv enablers will be now be discussed in more detail.

\subsubsection{Emphasis on the ICT Infrastructures}

The Corridor used its own intranet as part of its facilitating infrastructure to link participants. While this facilitates linking people to knowledge sources it does not necessarily realize the intended effect of establishing these linkages. Mullin (1996, p56) states that "The challenge lies in being able to train people to share and then to reuse knowledge within the organization".

Many companies worldwide have relied almost exclusively on big investments in documentmanagement systems, shared servers, intranets of different magnitude, and other technology solutions, believing this approach to be enough to let employees unlock knowledge (McDermott, 
1999). The result too often seems to be an overwhelming volume of outdated and poorly written documents leading knowledge buyers or seekers to sail in a sea of confusion and ambiguity.

Bryan argues that the real value of knowledge transfer at transnational level comes less from managing knowledge and a lot more from creating and exchanging it. He further argues that effectively exchanging knowledge on a company-wide basis is much less a technological problem than an organizational one: encouraging people who do not know each other to work together for their mutual self-interest (Bryan, 2004).

However, technological devices may also trigger the creation and exchange of knowledge on a face-to-face mode, provided that its platform facilitates a direct contact between the participants. Even though ever increasing technological dependence is evident in the logistics business, as well as in any other kind of large-scale PM operations, the Corridor's forums role has been to provide an exchange mechanism so that both authors and seekers of knowledge could find their way to a knowledge market. This forum which later became a knowledge market would have been much slower (or proved much less efficient in terms of knowledge sharing) without a minimum technology platform. This suggests that both a direct contact among team members and independent participants may be necessary initiating conditions to build trust and permit a knowledge market to emerge.

However, a key element to jointly trigger knowledge sharing and make it sustainable over time to allow this knowledge market to mature is provision for a common technological platform for participants to use. Technological sophistication, size, and structure of a company may indicate how ready it could be to utilize technology to facilitate creating and exchanging knowledge. However at the same time, these characteristics may conspire against and confound effective face-to-face exchange that might eventually lead to a more effective flow of knowledge.

A typical example of this is the giant American engineering company Fluor Daniel that develops projects across the Americas and all over the world. Even though the same team may be simultaneously carrying out a functional activity such as logistics on a number of different projects in distant locations, it is common for their members to not know each other despite 
having exchanged thousand of emails and have engaged in long hours discussing operations on the phone or in front of teleconferencing devices.

Dixon (2000) argues that technology used for knowledge work has to be married with face-toface interaction to create the most effective systems, and that even though one does not replace the other, one clearly enhances the other. Another problem that a technology-based knowledge transfer system often presents is given by the lack of willingness of some people in effectively transferring such a body of knowledge. Dixon (2000) further argues that when organizations find that their members show little interest in the knowledge or information repositories, they often have to expend considerable resources to provide (often by reinventing the wheel) the same common knowledge that could and should have been shared in an attempt to overcome the lack of contributions. Worse still, common mistakes may be constantly repeated. Thus, an incentive system is often designed and put into practice aiming at rewarding people for contributing and retrieving knowledge. Large and global firms such as KPMG, Chevron, Bechtel, Ernst \& Young, as well as other large consulting firms of global reach, have tried to address this issue through innovative incentive mechanisms, though mainly technologically-based KM solutions (Hansen, Nohria and Tierney, 1999).

Technology is no doubt a key element to efficiently manage knowledge as it allows people to share knowledge without having to be in the same place at the same time. However, this seems not to be the most efficient knowledge transfer method but that some sort of combination between technology and direct individual contact as experienced by Ford Motor Company through their "Best Practice Replication”. Here, the engineers from two different countries got to know each other to exchange views on how to improve their respective production lines; after face-to-face exchanges and discussions they came to respect each other, and to recognize that the other had some very useful ideas that they had not thought of themselves (Dixon 2000).

\subsubsection{Emphasis on the Human Infrastructure}

Experience with the Corridor indicates that face-to-face communications is the most efficient tool used by medium or small-sized companies to communicate to front-line employees while exchanging views and experiences in the MERSOSUR Corridor forums or integration 
roundtables. Face-to-face communication allows richer communication that includes senses of smell and touch that humans perceptively use when interacting. Larkin and Larkin (1999, p152) argue that "the best way to communicate a major change to the frontline workforce is face-toface”. This seems to indicate that technology can be best utilized by larger or more technologyuse mature organizations and when involving more analytical employees often localized at higher managerial echelons of the organization rather than at frontline workers who may not have the ICT skills to use high technology KM approaches.

The knowledge sharing and transfer literature discussed in Chapter 3, generally cites examples drawn from single large corporations that encompass a great number of worldwide divisions and does not often cite examples from regionally-focused smaller organizations embodying a great diversity of functional activities such as that often seen within the logistics world. This research thesis focuses upon how KM operates between both competing and complementary firms that are grouped under a common entity (the Corridor). This offers a fertile ground to further develop the existing knowledge management experience into a new situation that is poorly addressed by the current literature.

Most of the Corridor members and participants fall into the category of either small to mediumsize companies with a regional focus or larger and global companies whose regional branches operating as independent entities adapted their KM style to the KM advantages offered by the Atlantic Corridor.

It is been the author's experience that direct face-to-face dialogue was the preferred method for sharing valuable proprietary knowledge inside the Corridor forums, between knowledge providers and buyers, and this provided a pillar upon which tacit knowledge to explicit knowledge conversion was initiated. If knowledge seekers find a willing expert, they can quickly pinpoint and acquire the knowledge they need. Whether meeting with them one-on-one or in a group, the knowledge provider is likely to gain recognition and esteem from peers and superiors.

This introduces a culture matching dimension that is not further explored in this thesis. Detailed analysis from a culture perspective is not pursued due to limiting the scope of this work and because the context of the Corridor is within a Latin America with members of a similar cultural. 
The MERCOSUR countries fall within a similar band as reported by (Hofstede, 1991; Gupta, Hanges and Dorfman, 2002). I have briefly discussed various aspects of organizational culture in Chapter 3 Section 3.8. This cultural dimension from a national and organizational cultural aspect does, however, lead to recognizing that culture has an influencing role in knowledge sharing facilitation. Even more so if applicable to a Corridor-like organization which turns out to be different than a cooperative, consortium, joint-venture, or alliance of any sort while tending to be a spontaneous gathering of both complementary and competing organizations - where culturematching is restricted to facilitating knowledge to flow and transfer.

Further to exploring extensively beyond the literature discussed in Chapter 3 relating to organizational and national culture, it is important to define culture as a set of refined behaviors that people have and strive toward in their society. Culture, according to anthropologist Taylor, includes the totality of knowledge, belief, arts, morals, law, customs, and other capabilities and habits acquired by individuals as members of a society (Cleland \& Ireland 2002, p559). Put in other words and from an organizational viewpoint, organizational culture may be defined as the environment of beliefs, customs, knowledge, practices, and conventionalized behavior of a particular social group (Cleland \& Ireland 2002, p559; Schein, 1993).

Elashmawi \& Harris (1998, p87) provide a definition of culture that seems to be very appropriate to describe the nature of the Corridor. They refer to culture as the behavioral norms that a group of people, at a certain time and place, have agreed upon to survive/coexist. Despite the countries in the region shared rather homogeneous cultural patterns, it becomes necessary to deepen into certain cultural insights to better comprehend the different cultural environments these countries belong to. The principal cultural influences are manifested in the dimensions described by Hofstede (1991) and in the GLOBE study (House, Javidan, Hanges and Dorfman, 2002; Jesuino, 2002) that are outlined in Chapter 3. While there may be significantly shared assumptions indicated across the MERCOSUR nations by virtue of these cultural dimensions they can not be used as a tool for predicting behaviors. The cultural dimensions and their clustering, however, can be used to better understand how various individuals from cultural clusters may share a world view that helps them to readily relate to each other. 
People make organizations work, and the culture of the form of PMO ties people together, giving them meaning and a set of principles and standards to live and work by - a concept that is of paramount importance in the knowledge transfer process. From this perspective, the Corridor - as a corporation - may be imagined as a larger entity or master project made up of a number of project teams or groups holding different life cycles in their particular sub-projects, and where every group develops its distinct sub-culture - one where members are influenced by the culture of the organization to which the group belongs as well as separate project cultures. Corporate culture is usually explained in terms of organizational values and beliefs and the behavior of members of the corporation (Schein, 1993).

Cleland \& Ireland (2002 p560) argue that the culture of an enterprise and the culture of a project within an enterprise are mutually interdependent, influencing each other as the two organizations work together. A reengineering project which results in organization-wide downsizing, restructuring, and realignment of the way in which project processes are managed is also likely to have an impact on the local culture. Firms may also need to adapt the organization to fit cultural differences within a cross-company project meaning that both an institutional and individual adjustment is very likely to be needed at an early stage. The meaning and real extent of these concepts loom large if a trans-national characteristic is considered and embodied within a knowledge dimension - a topic this dissertation will intend to cover by assessing the roots and impacts with respect to this unique organization.

Davenport and Prusak (2000, p93) give various examples of various companies organizing workshops, fairs, and conferences under different degrees of freedom or flexibility for the executives to wander and interact in a more or less natural way, aiming at holding informal conversations or exchanging tacit knowledge. They argue that large firms that organize events in a rigid or too highly structured way hinder informal interaction and therefore limit the exchange of tacit knowledge. On the other hand, firms allowing executives free time to get in touch with each other turn out being much more effective from a knowledge sharing perspective. They advocate knowledge transfer through face-to-face meetings and through narratives in addition to more structured forms. Wandering and interacting are two essential constituting elements of the Corridor's flow of tacit knowledge. Naturally, giving people the opportunity to talk to one 
another does not by itself solve the problem of sharing and transferring knowledge. However, it is interesting to highlight the system adopted by a number of Japanese companies to let and foster knowledge transfer among their employees as a way to create value for the firm. They have set up "talk rooms” where informal and unscheduled meetings are held in order to encourage some kind of creative blending and exchange that eventually may bring about value creation. The Japanese corporate culture also encourages group dinners and after-work gatherings as a way to facilitate communications to spread out and to establish trust among the participants, without which knowledge transfer would not be feasible.

Nonaka and Konno (1998) introduced the Japanese concept of $b a$ or a space that has been especially created where learning can take place. The following actual examples from my personal experience illustrate how informal gatherings and fluid conversations are highly unlikely to contribute "per se" in finding an adequate response. These include conversations around: details of the design of a state-of-the-art Roll-on/Roll-off ${ }^{43}$ (RO/RO) vessel to carry automobiles within the MERCOSUR market; the optimal length and draft for a convoy of barges to carry iron ore ex central Brazil to a Buenos Aires up-river terminal throughout 2,400 kilometers of a tricky fluvial system; the best consumption/output ratio for a river tugboat to operate in the ParanaParaguay waterways; the most suitable management information system for integrating the regional railways by linking five countries; the optimal air-cargo freighter to operate economically under strong winds and long distance conditions across the Patagonian territories; the optimal lifting equipment for Manaos up-river port terminal in the Brazilian rainforest; the most suitable deck design for a project ocean-going vessel to call south American east cost ports; or the optimal emergency airplane capable to land and take off at 4,500 meters of altitude in a mining project's runaway of only 1,300 meters in length, turn out to be only some.

However, this informal gathering process is efficient in placing the early germ of ideas where these initial interactions may bring about promising outcomes at a later stage. Such outcomes - as will be described in more detail in the next chapters - may range from a simple cooperation agreement to a formal joint-venture agreement or even mergers and acquisitions between regional

\footnotetext{
${ }^{43} \mathrm{RO} / \mathrm{RO}$ is the technical term for those ships capable of loading, transporting, and discharging rolling cargoes such as pipe-lyers, side-booms, road-making machinery, mobile cranes, trucks and automobiles.
} 
corporations in pursuit of achieving a better corporate positioning that help them cope with the global trends more efficiently.

My experience and observations of the way that regional shipping companies reacted to the global trends represented by ever-growing economies of scale in the east-west-east trade lanes sheds some light on the way that face to face knowledge transfer occurred. Far from making contacts at corporate level, flying first class, or holding pre-announced overseas meetings, all contacts among the Corridor members were initiated in an informal fashion. Examples of two joint-ventures presented in Chapter 6 that later proved to be successful (and became a model to follow in lowering operational costs while rationalizing their respective fleets in operations), illustrate saw how "trust shaping” first steps in going forward in two different but both equally informal ways. The first example was during a friendly lunch between two shipping firms, and the second at conducting an informal meeting at the commencement of an owners meeting that proved to be totally fortuitous.

The first example was an ad-hoc lunch where key representatives of the parties got to know each other and explore the possibility of helping one another in coping with an upcoming threatening scenario dominated by lower-cost and larger-scale operators (Zim Lines and Maruba). The second example had the same purpose though an informal encounter that turned out to be crucial, triggered the face-to-face meeting that in turn helped cement a business relationship (Zim Lines and Pan American Shipping).

Little explicit data was exchanged thereafter, but that form of mostly tacit knowledge and face-toface dialogue and exchange of information became a feature of regular and frequent interactions between the executives and owners themselves. With the passing of time information and knowledge of the operational variables of each company was transferred to the other for their comparison and assessment (such as load factors / operational draft / consumption / load - unload speed / sales force / organizational structure / IT systems). Additionally, information and knowledge that not long before had been considered highly strategic information (port rates / 
market freight rates / service contract rates ${ }^{44}$ / bunkering strategy / time-charter rates ${ }^{45}$ / operational costs) was also freely exchanged. Thus the special characteristic of the Corridor's own developing ethos and culture of facilitating knowledge transfer and developing cooperative ventures was achieved through the Corridor's ability to enhance informal interactions where significant tacit knowledge could be exchanged and re-framed in the above example from 'highly confidential' to 'sharable and tradable' in this evolving knowledge market.

Face-to-face interaction also resulted in building trust and therefore better cooperation and cohesiveness among teams spread all over the world, when corporate sales meeting were held once a year. All those people with whom one had been in contact with on the company's intranet, finally got together, shook hands, started exchanging views and opinions on a number of common marketing topics, and most importantly, built a solid trust base. Knowledge transfer and cooperation tended to become increasingly easier after every meeting took place.

Communications were more fluid, understanding resulted more readily, tolerance for bad moods was higher, and overall cooperation became evident. This illustrated how the Corridor through these meetings, where people interacted face to face, were able to build trust and affective commitment as discussed in models and theory presented in Chapter 3 section 3.8.

Another aspect that is worth-considering in terms of knowledge sharing and transfer is the role that the emotional dimension plays in a typical interaction of parties within a very particular context into which business develops in South America. Nichols and Stevens (1999, pp8-14) argues that in different degrees and in many different ways, listening ability is affected by our emotions. When someone says what we especially want to hear, we open our ears and may accept everything - truths, half-truths, or fiction. On the other hand, if we hear something that opposes our most deeply rooted prejudices, notions, convictions, our brains may become over-stimulated and tend to reject that piece of information by creating emotional filters.

When the business environment mutates and shows little or no chance of being effectively anticipated, emotional filters seem to play its role in that Corridor member companies tend to

\footnotetext{
${ }^{44}$ Rates that are assigned to a given contract to carry a specific number of containers during a certain period of time from the port/s of loading to the ports/s of discharge.

${ }^{45}$ Rates that have to be paid for running a ship on daily basis and for a specific period of time.
} 
exchange both explicit and tacit knowledge in an almost desperate need to find an alternative action to consider countering any upcoming perceived business threats posed by the global economy. In order to avoid the growth of such emotional filters and to better deal with them, a proper understanding of the contextual culture and habits becomes essential. In this regard, it seems one may conclude that for letting tacit knowledge emerge and be exchanged in a Latin American environment, it may be necessary not only to allocate more free time and to support occasional gatherings, lunches and dinners, but to also implement informal brokerage events to actively encourage people towards interacting and sharing knowledge .

This is where a sound understanding of the regional culture particulars becomes essential, as it often plays a key role in easing or impeding knowledge sharing and transfer. Furthermore, existing emotional filters as referred to by Nichols and Stevens (1999), tend to be neutralized by the ever-fluctuating South American business context which makes knowledge sharing and transfer flow easily and be regarded as a matter of survival when facing the effects of globalization. In this sense we can see examples of continuance commitment driving affective commitment (Meyer and Allen, 1991) discussed earlier in Chapter 3 section 3.8.

An element that turns out important to knowledge transfer and is directly related to the Corridor's CoPs is what Burton-Jones (1999) describes as "sticky" knowledge and what Szulanski 2003) defines as its three constituting elements: absorptive capacity, causal ambiguity and the quality of relationship between providers and seekers of knowledge. Further to what was already described in the Chapter 3 section 3.7 about knowledge stickiness, it is useful to link this theoretical concept with some Corridor-related events that the author had the opportunity to witness when participating in the roundtables.

Examples of sticky knowledge became evident during the shipping crisis ${ }^{46}$ which led to entire fleet rationalizations and the setting-up of highly complex vessel sharing agreements ${ }^{47}$ (VSA)

\footnotetext{
${ }^{46}$ The regional shipping crisis took place in the late 90s owing to a growing overtonnage which resulted in an excess of supply along with a sharp fall in ocean freight levels further to continuous increase in the bunker prices. All this led shipping companies to reduce or "flag-out" part of their vessels while entering into joint-ventures and alliances with ex-competing firms to match the actual level of demand and better cope with their operational costs.

${ }^{47}$ Vessel sharing agreements were a more elaborated type of joint-venture agreement in which a third or even more parties could easily join should the other parties agree to. It also aimed to reduce operational costs and eventually
} 
among the world's largest firms. These agreements entailed a great deal of IT coordination to facilitate exchange of very specific and delicate knowledge possessed by a very limited number of people with highly technical backgrounds. These people had limited or no experience or practice of knowledge sharing or transfer (or even participating in team-work) with other firms executives participating in the Corridor engaged in collaboration and knowledge exchange. The difficulties encountered could be seen as a result of a lack of absorptive capacity of firms at the early stages of engagement in Corridor knowledge exchanges.

Cohen and Levinthal (1990, p128) define absorptive capacity as the ability of a firm to recognize the value of new external information, assimilate it and use it for commercial ends. They contend that building absorptive capacity requires long exposure to experimentation, trial, error, reflecting on lessons learned, and seeking out information and knowledge both from within the organization as well as outside of it. However, two distinctions are needed here with respect the way the Corridor's CoPs have dealt with it:

The context's volatility and uncertainty along with its rapidly changing business conditions - mainly in Argentina and Brazil - did not adversely affect the roundtables and CoPs. On the contrary, and beating the most pessimistic forecasts, the Corridor CoPs played a fundamental role (in my experience when compared to traditional non-Corridor practice) by shortening knowledge transfer times and optimizing results from these interactions. Even though there was no room for long experimentation periods, the assimilation process proved to be a potential model for other CoPs worldwide. This process brought about a significant number of joint-ventures, alliances, partnerships, and commercial or operational agreements of varying magnitude and impact without producing major market disruptions. The pace of the economic and political changes in the region can be judged as the best teacher on timing issues relating to decision-making. It forced executives to absorb what they come across with at great speed - making the concept of knowledge stickiness a highly relevant factor. It is instructive to highlight the reputation held across the Americas of both Argentine and Brazilian financial managers to anticipate and successfully navigate through recurrent financial crises. Similarly, economists

lower the exit barriers. These type of agreements allowed some regional owners compete at international level by joining existing outer regional VSAs. 
specialized in monetary systems from these two countries are also well reputed and sought worldwide. It seems that in their cases neither the lack of experimentation nor the possibility to assimilate information in due time (that one might well considered difficult to absorb) are impediments to a fast and effective knowledge sharing and transfer. Naturally, it seems to be the business context imposing its own rules of the game. Thus history and experience of coping with prolonged instances of business uncertainty and turbulence provided an impetus for developing high levels of absorptive capacity in facing the kinds of problems addressed by the Corridor's activities.

The process of seeking out information is more related to outside the organization and very little to within the firm. The existing CoPs have given many firms the unique opportunity to search for information outside their own firms and in addition to within the group they belong to at the same time. This special circumstance encouraged and drove a great number of executives to share tacit knowledge and let knowledge seekers and providers interact in flexible structures allowing knowledge to emerge and increasing the likelihood of prompt access to and interaction with externalization sources of knowledge.

Jewell and Walker (2004) argue that a consequence of poor absorptive capacity is often an inability to understand cause and effect loops leading to what Szulanski (2003) describes as causal ambiguity - the condition where people are not able to confidently make a cause an effect link and thus fully understand an issue. An example of the Corridor's high absorptive capacity and low causal ambiguity is illustrated in the following table:

Table 4.1: Absorptive capacity example.

PROBLEM

a) Wheat, corn, malt (Brazil)

- Wheat import restricted in Espiritu Santo .

- Low storage capacity.

- High costs.

- Annual freight volumes of 150.000 tons per year.

b) Port of Zarate (Argentina)

- Great dearth of knowledge of - Investment opportunity in port trade opportunities afforded by
ACTION

RESULT

- Included wheat in the modeling of - Lowered costs. port activities .

- Attracted investments in new silos. - Increased storage

- Attracted trade lane Ghent-Vitoria to 60.000 tons. for the distribution of Braham malts - Increased grain to the southeast region . throughput to 900.000 tons/year.

\footnotetext{
- Association Murchison-Cotia
} 
MERCOSUR on behalf of Corridor inland dry ports.

Consortia and MERCOSUR - Inland dry ports for vehicles countries

imports and exports .

- Investment in 2

berths and 100.000

square meters .

- Many opportunities if

- Project driven Murchison-

- New weekly

Information passed along

Cotia Trading between regions

service VIT-ZAR

c) High costs of storage

- Large volumes of cargo

- Benefit/cost analysis of single

- Regulation permitting imports through inland dry ports payment only at inland dry port issuance of customs transit declaration

- Storage surcharges doubling costs

- Warehousing is only paid in the inland dry ports, whereas cargo is delivered in $4 \mathrm{hrs}$.

- Expansion of imports restricted by high final costs .

d) Energy shortage

- Espírito Santo imports 80\% of its energy

- Joint promotion with the Government of Espiritu Santo for gas distribution
- Extension of pipeline serving 10 business sectors

\section{- Petrobras Oil Company is} deactivating its research

\section{- Additional} research resulting in the discovery of larger deposits

e) Malt logistics

- High costs of road haulage

- Create a new logistic system .

- Long distances

- Bring costs down

- Freight costs impacting on

- CVRD proposed building a rail manufacturing costs link $100 \mathrm{mts}$ from access to malt silos inside port

- Railroads without access to malt within the port, prohibiting modal systems to work

- Forums brought together Brahma

- Port Authority to build new rail link.

- Transfer of highway cargo to rail cargo

- First transport of malt via rail .

Source : Boske, L.B. (2001). Maritime Transportation in Latin America and the Caribbean.

Lyndon B. Johnson School of Public Affairs. The University of Texas at Austin; Policy Research Project Report \# 138 ; pp226-8 . 
One of the reasons whereby the Corridor's CoPs show a high absorptive capacity and low causal ambiguity from a knowledge sharing and transfer perspective, may be better explained by Livingston (1969:p85) when citing the scientific research undertaken by McClelland of Harvard University and Atkinson of the University of Michigan with respect to motivation (Atkinson, 1957). The research he cited illustrates a bell-shaped curve of risk taking behavior showing the relationship between motivation and risk taking effort. The degree of motivation and risk taking effort rises until the expectancy of success reaches 50\% then begins to fall (suggesting a satisficing motivation at that point) even though the expectancy of success continues to increase.

By looking at the Corridor's roundtables and bearing in mind the context into which these are immersed (where business conditions mutate both rapidly and frequently and therefore are highly charged with risk and uncertainty) it is not surprising that either success or failure arrives in such a short period of time that it allocates no physical time to let CoPs' members get either too excited or somehow indifferent as to produce a sharp fall in the motivation curve. The above suggests that the speed at which events take place does not allow motivation to fall, but on the contrary, to be always at or near the peak of the bell-curve. This is because the accomplishment of certain types of goals mostly involves a matter of survival. Once these goals have been attained then it comes a process concerning the way these firms can adjust and position their relative services with respect to the way they will face the new market structure by tackling one or various of the following strategies where motivation most likely conforms to a new bellshaped curve.

Table 4. 2: Alternative strategies STRATEGY DEFINITION KNOWLEDGE APPLICATION

Forward integration

Backward integration
Gaining ownership or increased control over distributors or retailers

Seeking ownership or increased control on a firm's suppliers
Sharing / Transfer (exporters / railways/ shipowners)

Sharing / Transfer (airline operators / ports / third-party logistics / shipyards / containers builders) 
Horizontal integration

Market penetration

Market development

Product development

Concentric diversification

Horizontal diversification

Retrenchment

Divestiture
Seeking ownership or increased control over competitors

Seeking increased market share through greater marketing efforts

Introducing present services and products into new geographic areas

Seeking increased sales by improving present products or services or develop-ing new ones.

Adding new but related services

Adding new, unrelated services for present customers

Regrouping through cost and asset reduction to reverse declining sales and profit

Selling a division or part of an organization
Sharing / Transfer (alliances / jointventures / VSA )

Sharing

(shipping / barging / railways / truckers/ airlines / airports / ocean terminals / insurers / bankers / ship-building and repairs)

Sharing / Transfer (shipping /railways/ airlines / exporters / importers / brokers)

Sharing

(logistic operators /

exporters /

importers/

bankers / ship-

building)

Sharing

(shipowners /

railways /

customs

brokers / coastal

Sharing

(third-party

logistics)

Sharing (shipowners /

truckers /

airlines /

railways

exp-imp / ship-

building and

repairs)

feeders / ports)

Sharing /Transfer (shipping / ports/ 
barging /

trucking /

warehousing /

surveying )

Liquidation

Selling of all company's assets, in parts, Sharing /Transfer

for their tangible worth

(barge operators /

railways /

shipping /

trucking /freight

forwarders /

brokers

Source : adapted from David, (2003). Strategic Management Concepts; Prentice Hall $9^{\text {th }}$ edition, New Jersey ; p161.

Table 4.2 suggests to me, given my experience in this logistics field, that even though knowledge sharing occurs throughout every and all of the mentioned strategies, knowledge transfer only takes place every time a vertical integration possibility opens up, a market development chance becomes real, or every time a company take over assets sale take place. Every time a tangible advantage presents itself for both parties, then strategic information barriers tend to vanish and lead to a fluid knowledge transfer (e.g. mergers and acquisitions). Knowledge transfer has proved being an essential element or a process enabler for letting alliances and joint-ventures successfully evolve in some sort of vertical or horizontal integration, as well as to foster mergers and acquisitions which helped regional firms either facing the effects of globalization or just benefiting from it by selling off and give up the logistics market.

The role that mentoring between participants may play in a Corridor-like organization with respect to becoming a knowledge transfer facilitator is important. Davenport and Prusak (2000, p95) suggests that tacit knowledge transfer requires extensive personal contact in the form of a partnership, a mentoring or an apprenticeship and that such a relationship entails transferring various kinds of knowledge, from explicit to tacit. Mentoring also has a link with leadership whereas the latter is essential to let a CoP be an effective knowledge management vehicle.

Zaleznik (1998, p62) argues that mentor relationships are crucial to the development of leadership personalities, but in large, bureaucratic organizations, such relationships are not 
encouraged. He further argues that working in one-to-one relationships - where is a formal and recognized difference in the power of the players - requires a great deal of tolerance for engaging in any emotional interchange. This helps explain one of the most common causes considered by executives in avoiding committing in such practices. Further, as explained in Chapter 3 section 3.8, culture, and power distance in particular, influences the nature of such interactions.

This mentoring process did not happen at the Corridor without first incorporating a gradual transformation process from formality to informality between working groups and CoPs. At the outset, while individuals within companies began to get to know each other and start to build trust and reasonable working and human relationships to let tacit knowledge flow, the forums merely resembled working teams of a very formal and structured nature. However, with the passing of time and with the reinforcement of a threatening business context approaching the region (where rules and variables often tended to shift drastically) such working teams' formality tended to gradually mutate to bring about - in many cases - voluntary and spontaneous CoPs aiming at exchanging and transferring knowledge on very critical issues and in a rather informal fashion. Examples of the context they worked in include: a business macro-environment of $40 \%$ devaluation; capital concentration on the railway sector; heterogeneity in the regional customs procedures; raising rate of cargo claims; new tax regimes on shipbuilding and ship-repairing; Central Bank restrictions on overseas money transfers; and double currency accounting legislation required for foreign firms..

Skyrme (1999) refers to seven steps companies have to follow to secure strategic advantage from a knowledge perspective:

1. Customer knowledge;

2. Knowledge in products and services;

3. Knowledge in people;

4. Knowledge in process;

5. Organizational memory;

6. Knowledge in relationship; and

7. Knowledge assets 
From the above points, I argue that there seemed to be little doubt that the forum roundtables around the region fulfilled at least 6 of the above recommendations, though attaining strategic advantage will always be conditioned - to a great extent - to the business environment or the accuracy with which business trends could be anticipated. Knowledge in customers, products and services, people and processes, relationships and assets can be said to smoothly flow in the forums every time the roundtables were held. Organizational memory, however, intends to adapt to better process the information in a way that helps avoid overloading the system with information of relative low value. Access to organizational memory is neither free nor equal, but remains restricted and conditioned to the level of participation and interest of the members.

It can be argued that the richer the tacit knowledge becomes, the more technology should be used to enable people to share that knowledge directly - which creates a sort of interdependency between what is been sustained so far with respect to face-to-face interaction and the utilization of a technology platform to facilitate the set-up of a knowledge market In this regard, the Corridor's on-going intranet system linking the many forums at regional level plays a key role in facilitating codifying and disseminating knowledge for all those knowledge seekers who intend finding useful guidelines to deal with specific problems. However, face-to-face contact in the forums remains as the triggering factor for knowledge sharing to occur, technology being a valuable knowledge sharing and transfer enhancer but of secondary importance.

An efficient knowledge sharing and transfer system seemed to need to consider a number of factors (both technical and cultural-related) if an appropriate system design is aimed for. These include a dynamic face-to-face interaction / a suitable technological platform / assessing the actual absorptive capacity and causal ambiguity dimension / measuring the stickiness of knowledge and identifying the potential emotional filters / having a good picture of what the triggering factors are as well as the existing motivational level, etc. However, none of these variables would display coherent or cohesive results without the existence of a truly cooperative spirit among the participants - which according to what it has been developed so far seems to be very much driven, or perhaps imposed by, a critical regional business environment i.e. economic triggering motivational factors. The next section will more deeply explore the concepts of cooperation and collaboration in an intent to help the reader better comprehend the extent to 
which these two concepts are of paramount importance in shortening the time to let knowledge emerge and spread out to generate innovative outcomes.

\section{3 - COMPETITIVE ADVANTAGE THROUGH COLLABORATION AND COOPERATION}

This chapter argues that face-to-face interaction seems to be of paramount importance in the emergence of knowledge sharing and transfer. It also argues that associated technology should enable or facilitate knowledge transfer rather than be a system upon which the entire concept of knowledge management should be based. However, there is an active role played by this technology that goes well beyond the concept of knowledge sharing and transfer. Such a role has to do with the very reasons by which companies become global and engage themselves in achieving larger economies of scale and broader market coverage.

This important technology driver that relates to the competitive advantage role of technology rather than just facilitating knowledge management will now be discussed in detail. Schlier, Hunter, Harris \& Berg (1998) argue that information systems executives and professionals that understand the business drivers of technology demand will be asked to participate in enterprise planning and will be expected not only to align technology investments with enterprise strategy, but also to help enterprise business leadership understand how to use technology to align the enterprise within a dynamic marketplace. They further argue that the emerging global economy will be a major driver of enterprise strategy formulation and technology application in the years to come. Equally so, improvements in technology, combined with dramatic changes in geopolitical economics, have been major drivers of the globalization of competition. Such a globalization process (as discussed in Chapter 3 section 3.2) along with the development of technologies in its broad sense may be regarded as a prime triggering concept toward regional cooperation and collaboration.

In the opinion of Schlier et al. (1998) there are a number of foundation technologies that have most affected the globalization of competition:

Engineering;

Materials; 
Manufacturing;

Transportation;

Information;

Communications; and

Entertainment.

Transportation, information, and communication technologies seem to have played a crucial role in transforming the MERCOSUR region by granting a number of big firms the needed technological means to enlarge their geographical reach. The deployment of their resources throughout a less technologically advanced region gave them a strategic advantage that automatically brought about a defensive reaction in the form of the Corridor. It has been argued in this thesis in Chapter 3 that technological improvements have combined with political and economic factors to drive the global economy and lead to changes in both politics and economics. These changes opened up significant market enlargement opportunities in a number of forms:

o Changing political and economic models;

o International currency agreements;

o $\quad$ More liberal regional trade agreements;

o $\quad$ Patent and copyright agreements;

o The changing role of government;

o $\quad$ Reduced economic risk;

o $\quad$ Reduced political risk; and

o $\quad$ Reduced business risk.

Typically, the economic, political and business risk of an already risky and unstable region (MERCOSUR) turned out to be of a lesser relative significance than the risk comprised in the corporate decision to remain active in more stable markets instead of operating in South America. Not having presence in South America would have meant for some of the larger world players simply loosing ground in their struggle for enlarging economies of scale and gaining competitive advantage. Therefore, it may be argued that the lowering of entry barriers in South America acted both as an opportunity for the global players to become even more global (though it seems this was not an optional choice for them to select but that it became a vital one to maintain a low 
global cost base) and a strategic avenue for some regional players to also expand their services and lower their cost base while acquiring knowledge.

Porter (1990) highlights the central role a home nation plays in a firm's international success (as discussed in depth in Chapter 3 section 3.2). His argument helps make sense of the steps taken by a number of maritime nations' shipping companies that expanded globally until reaching the MERCOSUR coasts. These include Denmark's Maersk and Lauritzen, Norway's Gearbulk, England's P\&O, Germany’s Hamburg Sud, or Holland's Nedlloyd. However favorable the national circumstances may be, success is not ensured. Global competitive advantage results from an effective combination of both national conditions and company strategy even when conditions in a nation may create an environment in which firms can achieve this advantage. Porter (1990 pp578-84) enumerates a number of competitive advantage triggering factors as explained in depth in Chapter 3 Section 3.3, that I expand upon below as it relates to the MERCOSUR:

Competitive advantage grows out of improvement, innovation, and change: innovation leads to competitive advantage when a firm perceives an entirely new buyer need or serves a market segment that others have overlooked. This may be the case of America's river operator Crowley that positioned its state-of-the art river tugboats and river container ships to operate in the Parana-Paraguay waterways linking four South American countries in a way that no other had tried before. Here, a competitive edge was given by improvement, innovation and change through service speed, use of navigational equipment and cargo tracing devices, and financial standing. Another example is England's P\&O container terminal in Buenos Aires which became South America's most modern facility (after overcoming an endless list of both institutional and operational barriers) and a model to imitate at regional basis. Typically, load and discharging speed, storage facilities, advanced cargo tracing equipment, and modern multimodal transportation systems imposed a remarkable competitive advantage in a segment where outdated and inefficient competitors previously prevailed. Brazil’s ALL introducing the double-stack concepts in their railway wagons that also imposed a technological and service innovation that brought about competitive advantage into the market. These two last cases clearly brought operational improvements, managerial innovation, and change in the way their respective markets started to be serviced. 
$>$ Competitive advantage involves the entire value system: it entails the participation of an entire array of activities connected with the various logistic segments (ocean transportation / river transportation / port terminals / air-cargo operations / railways / trucking companies / warehousing and distribution / surveying / crating / ship-building / logistic firms / ancillary activities). Value chains tended to mutate rapidly and either adjust to the needs imposed by the new global players across the MERCOSUR, or to reorganize through cooperation and collaboration to better cope with the concepts of improvement, innovation and change coming from overseas. Corridor members' value chain combinations were of paramount importance in helping these firms adopt a defensive strategy before a threatening scenario dominated by larger economies of scale, more advanced technology, superior financial capability, and innovative management. All these concepts were imported from the global firms' home base which tended to reorganize and adapt to regional needs.

$>$ Competitive advantage is sustained only through relentless improvement: unless protected by national legislation (an issue that is no longer existent within the Corridor scope of activities) so that firms that remain a stationary target are eventually overtaken by rivals. Permanent improvement was neither necessary nor imposed by a given market phenomena prior to the arrival of the global players in search of enlarging economies of scale and market expansion. Pressure for more and sustainable improvement was a byproduct that emerged as a consequence of the global players positioning their services and setting up their systems in a region where both, technology and service, had plenty of scope to outperform regional players' practices. These services ranged from coastal shipping to airfreighting and port operations and involved the world's largest firms by segment.

Sustaining advantage demands that its sources be upgraded: foreign competitors can imitate procedures and acquire the needed technology or make use of the necessary facilities to successfully gain regional market share within the MERCOSUR. Operational costs or logistic designs can be easily replicated and even upgraded once suitable market information becomes available. Even regional well reputed brand names, can be easily surpassed by global brand names that become rapidly known in the regional market. Further to this, and despite knowledge is supposed to remain in the minds of the holders, traditional knowledge could also be hired and grabbed form the regional firms to the 
advantage of the newcomer global players. Global firms like Maersk, NLL, P\&O, SeaLand, Evergreen, COSCO, APL, NYK, Hutchinson, and many others, tried first to gather all the available market information to later scan the market to capture whatever traditional knowledge they deemed it necessary. Naturally, the objective was to facilitate their entrance to a new market by shortening their induction time. This was possibly the most significant triggering element toward the enhancement of collaboration and cooperation amongst the many Corridor members.

Sustaining advantage requires a global approach to strategy entails coordinating and integrating functions on a worldwide basis in order to: gain economies of scale or learning; enjoy the benefits of a consistent brand reputation; and serve international customers. All these concepts drive regional firms to unite and seek a joint strategy that helps them face an overwhelming business context in order to restructure or redirect both their individual and joint competitive advantage profiles. Typically, home base strengths were mainly represented by utilized technology, carrying capacity, global customer base, financial back-up, global supply chain, and organizational advantage.

It becomes apparent that in view of the above named competitive advantage-related topics, the Corridor was urged to reorganize and its members to quickly adopt a number of defensive strategies. These were taken step by step, and on a gradual but steady basis, leading to an evolving state of business cooperation and information exchange collaboration. Such a participative or open-minded corporate attitude was not born per se, but was grounded by a number of topics that became the Corridor's objectives to be attained in the short run. These can be summarized as follows.

Reducing transaction costs: It became essential for shipping firms to achieve lower port handling fares and terminal costs in order to successfully match those of the global players. Lower ramp services and distribution costs were also key variables for regional air-cargo carriers to achieve to remain competitive with those of the global airlines which deployed more frequent services and greater carrying capacity. Railway operators had to increase their rolling stock and upgrade their storage and door-to-door delivery facilities to compete with the barging operators while at the same time reducing their operational expenses by complementing the global shipping companies' inland transportation 
necessities. Coastal feeders had to both rationalize and join their fleets in order to lower their ports costs to compete with main liner calls that were disputing their market segments. Container trucking firms also had to upgrade their assets and join fleets and management to better procure their diesel supplies and spare parts to be able to cope with both the global and recently reengineered regional ship-owners and the global outsourcing ocean carriers' lower relative pricing. Railway and port terminal operators were also similarly affected. Cooperation and collaboration in terms of supply chains, cost matrixes, operational management, and utilized technology became strategic issues to reducing costs and face globalization.

Obtaining greater volume and presence by reaching agreements with suppliers, competitors, and customers: As detailed in Chapter 3 Section 3.3, the global threat triggered both cooperation and collaboration to emerge as well as regional rivalry decline. Those who in the past were regarded as long time rivals became today's strategic allies. These allies came from suppliers and competitors alike, and found a number of examples of strong customers of regional reach too, who viewed themselves threatened by an aggressive and global competitive shipping that could facilitate their foreign competitors make use of their global contracts to effectively reach distant markets. Achieving larger economies of scale by entering joint-ventures and alliances through cooperation and collaboration would allow these firms increasing their bargaining power while rationalizing their assets. Similarly, cooperation between suppliers and customers became of outmost importance in their struggle to adapt to a new volume and cost-based scenario.

> Seeking efficiency in particular strategically significant activities: Cooperation and collaboration resulted in market segmentation where achieving a high level of specialization would lead to gain competitive advantage and consequently raise higher relative entry barriers. Coastal feeding services between Santos and a series of northeastern Brazilian ports, for example, involved both long distances and a high customer loyalty and proud with respect to national carriers. Other examples illustrate this environment. Buenos Aires up-river navigation up to distant Paraguayan and Bolivian ports involved complex operations and high levels of distrust towards foreign operators. Coastal feeding patterns between remote Patagonian ports resulted in high value for customer-carrier loyalty and trust in local-based operators. Provincial ground 
transportation cooperatives turned out to be unsurpassable with respect to their market influence area. These are examples of a specialization effect through attaining operational efficiency from cooperation and collaboration in those segments. These also tended to result in high entry barriers that dissuaded the global players to enter these markets. Collaboration and cooperation amongst the various operators brought about a better market coverage at regional level. It also made gaining market knowledge a difficult commodity to imitate or acquire for those outside the collaborating participants.

- Utilization of partner's assets: Rationalization was perhaps the most important and first evident outcome of the cooperation and collaboration process undertaken by the Corridor members. No matter which involved segment is considered, it enabled logistic operators to dispose of their older and less efficient assets and share their most modern and less costly assets with their new partners. The assets to remain in service generally led to deploying higher service frequency, faster load/unload operations, more adequate speed/consumption ratios, more balanced tonnage supply with the present and projected demand, and a more efficient management of a wide array of operational assets resulting in reduced running costs. This process brought about a general upgrade of the regional logistic operators through cooperation and collaboration as well as adjusting their physical means in a more realistic manner to reach actual market potential. Thus, while the competitive threat of the entry of global players was acknowledged, it was going to prove being more lengthy and costly to those global players than at first appears apparent.

Corridor members, through their cooperation and collaboration, professionally and more efficiently coped with the arrival of global players and their larger economies of scale. The Corridor market logic also proved to be a valid strategy to dissuade some of these global firms to expand even further into some provincial or inland market niches. When the global operators decided not to enter a given secondary or provincial market, a new way to face the market became evident. Larger operators now had to deal with a solid group of logistic providers to outsource their needs rather than use a number of isolated or disbanded companies they would have expected to find prior to the formation of these players joining the Corridor. 
In this particular situation, rationalization and regrouping of firms through cooperation and collaboration proved to be a very powerful set of tools to offset the influence of the global players. This also resulted in their helping to improve local logistic market business prospects through being more efficient and allowing new business opportunities emerge. This new current of business opportunities actually arose out of the global capacity of the global players to achieve larger economies of scale and therefore lower operational costs producing conditions that tended to foster imports and allowed some export products to reach distantly located destinations that otherwise would have not be feasible owing to previously higher relative ocean freight rates or air fares. Thus the transformations triggered by the Corridor's actions in response to the business context seemed to instigate greater trade and economic activity.

Consequently, the global players were dissuaded to step in the relatively smaller volume provincial or local markets whereas in the larger and more voluminous and perhaps anonymous markets, the regional operators saw themselves compelled to restructure the available assets and upgrade their on-going managerial concepts. The barriers that naturally arose out of the cooperation process helped build a stronger competitive advantage amongst the Corridor members by enhancing specific knowledge, relating to competitive advantage issues, increasing bargaining power, incorporating allies within the value chain, enlarging economies of scale, and improving both the utilized of technology and existing managerial capacity to effectively react to the business environment. This has been a naturally evolving case for some distant provincial or local markets where the global players experienced higher relative entry barriers and where outsourcing proved to be more reasonable because of the above mentioned reasons.

The main Latin American markets however (e.g. Buenos Aires, Santos, Sao Paulo, Rio do Janeiro, Valparaiso, Santiago) had lower relative entry barriers and were more susceptible to allow external larger economies of scale and greater competitive advantage that favored those who were ready to deploy their global player advantage. In this case, regrouping around the Corridor, together with a decisive willingness to cooperate and collaborate within a wide spectrum of corporate topics that the Corridor offered, proved to be not as efficient or fast trackoriented as was the case with the provincial or local market examples. This is because trading volumes were not large, local operators showed more unity, and traditional knowledge was 
definitely a factor that was unlikely to be readily imitated. Consequently, these global players were more difficult to persuade to enter a market place where the relatively smaller provincial or local markets volume existed.

Regional operators, however, saw themselves compelled to restructure their available assets in their larger and more voluminous, and perhaps anonymous, markets and to upgrade their ongoing managerial strategy and attitudes to rivalry and knowledge, and reshape an entire market to better cope with the global firms. In other words, this group had to reengineer their business through cooperation and collaboration though in a relatively slower path when compared to the provincial firms who already knew each other thus enabling trust to spread out more rapidly. This illustrates an interesting tension between the more global-centric large centers and the more regional centers where different dynamics and business imperatives prevailed and so different responses and rates of adoption of Corridor possibilities were evident.

An explosion of business opportunities became viable as a result of the Corridor concept that permitted the formation of a plethora of alliances and collaborations of various forms. This resulted in:

- customs brokerage firms expanding into the freight forwarding business with the intention to gain control over cargo routing; freight forwarding companies and logistic operators opening customs brokerage departments as a way to provide more integral services which otherwise would turn them vulnerable to loose to the more integrated global firms;

- ship-owning firms disposing of their contracted shipping agencies and establishing their own shipping agencies to reduce costs and become more efficient by gaining control over their supply chain;

- shipping agencies regrouping and offering new or additional logistic-related services as a way to face both the new trend of the regional shipping firms and the global impact exerted by much larger firms; regional port terminals expanding into the door delivery services business ( delivery from supplier to end customer) and added cargo-tracing technological devices to better tracked what items they knew global terminals were bringing from their home base and worldwide operations; 
- regional port terminals reengineering themselves to specialize in a given segment rather than trying to embrace various segments simultaneously;

- shipyards tending to specialize in those segments where they not only could show comparative strengths but also where they might benefit from supplying the global players' needs in terms of new-buildings and naval repairs; and

- state and municipal governments realizing that they had a tremendous opportunity to become the preferred location for either regional distribution services or road transit purposes before both the regional reengineered group of companies and the global newcomers who were interested to expand their services through the identification of suitable bi-oceanic land-bridges ${ }^{48}$ and inland nodal connections ${ }^{49}$.

None of the above would have been feasible without a thorough understanding of global trends to enable the correct interpretation of the business context, as well as the willingness on the part of the great majority of the national and regional players to remain in business. It seems to be clear that the globalization process challenged what used to be a rather protected and isolated regional market and that it was precisely this threatening scenario that actually acted as a triggering toward setting up the Corridor. However, it also becomes apparent that none of the above had been possible either, if cooperation and collaboration would have been restricted somehow or not fully developed amongst all the Corridor members. It is important to understand the order in which the events took place (globalization > context interpretation > internalization > grouping >cooperation/collaboration) and the way cooperation and collaboration led knowledge sharing to emerge and later be transferred.

This process was not born just because it was the culture of the regional companies to become better and more efficient, but that a global threat was the real cause that was challenging the very existence of their businesses. However, and as it will be explained in the coming section and chapters, such a process produced an interesting variety of value chain interactions which through

\footnotetext{
${ }^{48} \mathrm{~A}$ bi-oceanic land-bridge is a transportation corridor linking two locations lying on different oceans. Typically, the Corridor grouped companies that were engaged in the transportation between Buenos Aires on the Atlantic and Valparaiso on the Pacific. Similarly, Puerto Montt on the Pacific and Puerto Madryn on the Atlantic in the Patagonian region.

${ }^{49}$ A nodal point or connection is a inland depot where cargoes coming from a large are of influence are gathered, classified and re-routed to its final destination. Typically, this set-up counts on all the needed handling equipment, infrastructure, and customs clearance facilities.
} 
cooperation and collaboration brought about not only a much more refined regional logistic marketplace, but also presented an invaluable opportunity for some national and regional players to become truly global firms.

Consequently, it can be argued that the globalization process was not only a strong action leading to a strong regional defensive reaction, but also it opened up a new business opportunity for a number of regional firms to become more international by entering in joint-ventures and global alliances. This would not otherwise have turned out to be possible in view of the region's business, geographical, historical and cultural structural limitations.

\section{4 - THE CORRIDOR AS A VALUE CHAIN}

Vertical integration, both forward and backward, relies upon many valuable examples among the Corridor members' value chains. As it has been earlier explained in this chapter and in Chapter 3 section 3.3, this has been the outcome or a reaction of these firms' necessity to gain competitive advantage as a way to face much larger and resourceful global competitors. These global newcomers managed to bring down the existing entry barriers to a South American market in view of their more advanced technology, worldwide customer base and larger economies of scale. Most, if not all, newcomers had already gone through a vertical integration processes at home that tended to upgrade their competitive profile long before the Corridor was established. Many of them have gone through horizontal integration processes too ${ }^{50}$.

This meant that these global players (e.g. shipping companies / ocean port terminals / shipbuilding and naval repair yards / NVOCC ${ }^{51}$ / air-cargo liners / barge operators / chartering brokers / insurance firms / legal consultancy firms / ground transportation and distribution firms had already gone through the process of value chain interaction leading to a more generally competitive industry. This process brought about a steady path of vertical integration (backward and forward) that tended to strengthen out those functions that were wrong within their value

\footnotetext{
${ }^{50}$ Typically, mergers and acquisitions in the ocean shipping, port terminal, and air-cargo businesses along with their respective inland distribution services.

${ }^{51}$ NVOCC: Non-vessel Operator Common Carrier means a company holding a given volume of business (cargoes) that charters from a ship-owning company part of its carrying capacity.
} 
chains, whereas the south American market remained somehow dormant or protected by bilateral treaties and restrictive national and regional legislation.

It is useful to refer to Porter's (1985, pp278-87) sources of global competitive advantage and impediments to global competition to better visualize the differences in terms of operational capacity and market scope between the global firms and the regional companies. These gaps became evident at the outset of the logistics globalization process when the regional players saw themselves compelled to face the more resourceful and technologically advanced global firms within their own territory or market domains in South America. This can probably be regarded as the triggering factor by which the Corridor. In broad terms, it became a need within the region as a forum to consider value chain interactions through knowledge sharing and transfer.

As discussed in more detail in Chapter 3 section 3.3, Porter (1985) distinguishes between sources and impediments to global competition. The former highlights the causes by which a company can become truly global, while the latter refers to the main reasons why a firm may find it extremely difficult to overcome barriers to go beyond its national or regional market. Naturally, the first group embraces the global companies whereas the second group refers to the firms operating within the MERCOSUR region. Porter's (1985) sources of competitive advantage are illustrated in the following examples that summarize the essence of the intent of the Corridor to be a unique model of a program of works or portfolio of projects that respond to an urgent and regional need:

1. Comparative Advantage: When a country or countries count on significant advantages in factor cost or factor quality, these countries will most likely be the sites of production and exports. Put in logistic terms, there is a significant comparative advantage in those ocean transportation companies that at home have developed a remarkable track record both from a cost and quality perspective. Denmark-based AP Moller (Maersk) is possibly the world's largest full-container shipping line that originally based its strength on both an old country shipping tradition and being a country geographically located in the heart of large European trade currents. This company has sustained a progressive vertical integration of both suppliers (backwards) and logistic distributors (forward) up to completing its entire value chain. Shipyards; container manufacturing; off-shore oil 
rigs; supply vessels; freight-forwarding companies; air-cargo freighters; insurance firms; university; ground transportation; ocean port terminals. A similar example is represented by Taiwan-based Evergreen. This is the world's second largest fullcontainer shipping fleet that originally built its strength on its country's highly open economy that enabled it to develop growing cargo volumes that formed part of the very essence of Taiwan's success in world trading. These two firms first developed on local and regional basis driven by both their countries and respective regions growing trade flows and later expand into the global market. Germany-based Hapag Lloyd is also one of the largest full-container shipping lines that grew through its home country's worldwide export and import potential. This firm's integration finds more correlation with commercial activities such as freight-forwarding and brokerage activities; ground transportation and distribution; air traveling business, as well as river transportation. Mexico-based TMM is Latin America’s largest full-container company whose vertical integration is mainly represented by two large railway companies across the USA and port terminals in Mexico and abroad- where large volumes of cargo have to be carried within the NAFTA ${ }^{52}$ region. Hong-Kong based Hutchinson is one of the world's largest port terminal operators with a great number of port terminals around the world. This firm grew larger and larger by taking advantage from its very home base where large volumes of cargo flowing in and out of the island shaped the ideal location to initiate this long learning process that later expanded worldwide.

2. Economies of Scale: All the above mentioned companies as extended their respective economies of scale well beyond the size of their respective national and regional markets. This process was facilitated by the internationalization process a great number of national shippers went through as a consequence of seeing also themselves in the strategic necessity to expand internationally and enlarge economies of scale. Examples abound. England-based P\&O acquired Holland-based Nedlloyd and expanded dramatically its scope of operations and cost base; Denmark-based Maersk acquired USA-based Sea-Land achieving an even deeper share in the American market to later also take over P\&O; Canada-based CP Ships acquired US-based Lykes to better cope with the NAFTA region though it later became part of Hapag Lloyd that acquired both

${ }^{52}$ NAFTA: North American Free Trade Association ; it includes Mexico, United States and Canada. 
firms; Germany-based Hamburg Sud bought Brazil-based Alianca to exert certain dominance around South America; Brazil-based railway operator ALL also acquired two large Argentinean railways as a way to integrate the two largest regional economies under a single business unit. Not only vertical integration was a factor in all these but also horizontal integration played a fundamental role in making a number of companies doubling their economies of scale overnight and therefore posing a major threat to a region unable to react accordingly. Expanding market reach, was a priority for these large firms as well as a tangible threat for a number of companies that had great difficulty in matching the global players' costs and service level.

3. Global Experience: Logistics means mobilization of resources and consequently the global experience in logistics represents the cumulative and comparative experience coming from a great number of markets or countries where a firm operates. A regular shipping line may embrace twenty or thirty countries displaying unique trading, operating and cultural particulars. Unlike exporting multinationals whose main business consists of exporting to a great number of countries from a few strategically located plants, shipping multinationals have to have both a headquarter in its home country and a great number of branches in all those ports their vessels call at. Here, global experience becomes vital in gaining competitive advantage as it brings tremendous managerial flexibility for these large firms to adopt the on-going regional modalities and to adapt their resources accordingly to what is specifically needed in every particular country or market.

4. Logistical Economies of Scale: Supplying many national markets often means that fixed costs can be spread and variable costs can be reduced in view of expanding carried tonnages which otherwise would tend to remain on the high side. Having a sound container fleet administration is often considered a healthy practice in the container shipping business while counting on large economies of scale sets a significant cost difference. Global companies tend to engage in long term volume-based container leasing agreements where the leasing cost per unit tends to be significantly lower than that of the regional carriers - who in view of their smaller relative volume and limited 
geographical scope become uncompetitive. Trailers and low boys ${ }^{53}$ are to a great extent hired from specialized firms that offer their equipment at the main ports of entry. Thus, those enjoying the larger volumes and tonnages will be the ones setting a favorable cost advantage. Once it becomes part of the pricing formation process it becomes very unlikely for the regional company to effectively compete, unless they also engage in vertical integration to enlarge economies of scale and deepen their competitive profile.

5. Marketing Economies of Scale: Counting on a global customer base is probably one of the main marketing advantages the global firms may count on as it provides them with an available critical mass right after having entered a new market. Furthermore, showing some degree of vertical integration makes it more possible for these global players to target a specific type of account that need a wider scope of geographical reach to expand their exports. This is been the case of those regional exporters having a significant output volume that prioritized the utilization of the global carriers in view of their lower cost base (which translated into lower ocean freight rates) and wider geographical reach (e.g. ARCOR's confectionary / SANCOR's dairy products / Odebrecht's construction machinery / PETROBRAS's oil and gas upstream and downstream / TECHINT's pipes). By means of the utilization of two and even three ports of transshipment and a lower relative slot cost (only possible through very large economies of scale) shippers could successfully reach distant markets for their exports and supplies, which otherwise would have been too costly and therefore unviable. The use of more modern technology also set a difference in the port terminal business in that the loading and unloading speeds, storage utilization, berth length throughput, transfer productivity and other indicators tended to keep the port's net occupancy rate (NOR) ${ }^{54}$ at a reduced level. Thus, vessel rotation resulted in faster cargo turnaround and port costs tended to diminish. Naturally, the global carriers were the preferred customers for the global port operators in view of their faster operations deployed during the loading and unloading operations were generally a more efficient operation.

\footnotetext{
${ }^{53}$ Low-boy is a special truck that allows carrying extra-dimensional cargoes in accordance with the on-going road transportation legislation in most of the countries.

${ }^{54}$ The Net Occupation Rate is defined by the period of time between a vessel commences unloading operations until she completes the loading operations.
} 
6. Economies of Scale in Purchasing: The global operators, no matter what segment they operate in, enjoy a number of competitive advantages that the regional ones lack. These advantages are represented by what is possibly the most strategic input (Fuel Oil) and the second most important (the charter market) ${ }^{55}$. As well as bunkering and hiring of vessels, other inputs become essential and set a cost difference in favor of those holding more global operations and greater bargaining power: dry-docking / crewing / trucking / distributing / tracing / warehousing / transshipping / barging / etc. No matter whether it is the primary or the support activities of the value chain the ones setting a cost difference based on larger economies of scale. Either by outsourcing or vertically integrating, the above represents a strategic tool for global companies in their permanent search of competitive advantage as well as it means a challenge for the Corridor companies to offset these impacts by combining their value chains.

7. Service Differentiation: Global ship-owners highlight their geographical scope of services and faster transit times by linking South American east cost ports with inland locations in India, East Africa or the Middle East by making one or more transshipments and after engaging with combined railing and trucking transportation. Regional railway operators stand out because of their long distance and door delivery capabilities all along South America's east cost and across Argentina until reaching the Pacific Ocean as a way to get a number of European ports closer to Chile (through combined land-bridge operations), as well as a number of Australian and southeast Asian ports closer to Argentina and Brazil (truck-rail modus). Global air-cargo carriers set a difference in terms of cargo carrying capacity, loading configuration, frequency, and inland connections towards eastern Europe and northern Africa from a number of hub airports located across Europe; river operators remark their relative faster sailing speed resulting in shorter transit times and greater frequency; foreign-owned regional trucking firms advertise their home base knowledge of servicing global companies to intend capturing accounts other than those they service at home. The service differentiation trend sustained by the global firms proved to be one of the major drivers

\footnotetext{
${ }^{55}$ The time charter market is where most liner shipping companies hire part of their fleets until their new-buildings are completed at the shipyard and effectively deployed on service. This market is often utilized to cope with a specific market boom which otherwise would become unviable in view of the time lag involve in ship-building.
} 
for the regional companies to join the Corridor in search of improving their value chains and face these threats with a valid alternative.

8. Proprietary Service Technology: Porter (1985) argues that global economies can result from the ability to apply proprietary technology in several national markets when economies of scale in research are large relative to the sales of individual national markets. This seems to be the case of those large freight forwarding companies holding a dominant position not only in their large home markets but also in a number of regional and overseas nations. Germany-based Rohde \& Liesenfeld, Kuhne \& Nagel, and Schenker; Switzerland-based Panalpina and Danzas; Italy-based Merzario; Francebased Saga; USA-based TNT or DHL, are just a few examples of those global firms that developed their respective intranet systems to better trace their cargo flows around the world. All the shipping companies mentioned earlier and all the global port operators that later entered into the south American market, have researched extensively and developed their respective intranet systems to let their customers freely log in and find whatever piece of information they need to identify their cargoes. Before this complex scenario the Corridor was close to an ideal forum to let some of the regional members upgrading their support activities and therefore, also their entire operations.

9. Mobility of Services: This aspect represents the very nature of the logistic business in most of its segments. The more ships a company owns, the more chances it has to cover a wider geographical area. The more cargo-aircrafts a firm owns, the more global it may become. The more hub ports spread out in the world a port terminal management firm owns, the higher their chances will be to redirect world cargo flows. The more global a logistic firm may present itself before their customers the better their chances will be to control cargo routings. The longest the reach of a railway operator, the greater their chances to link distant points and reduce the unit cost will be. The greater the number of available trucks, the more chances to become outsourced will be. Naturally, the concept of mobility is common to all operators, whether large or small, national or global. However, the number of assets holds a direct relationship with the potentiality to become global and therefore achieve larger economies of scale and gain competitive advantage. Even though owning a large number of assets does not allow a firm to become automatically global, it generally means it may become so if it wishes. 
Switzerland-based Mediterranean Shipping Company is a rare and good example of this as it is possibly the only global ocean shipping firm that did not go through any horizontal integration process and relatively little vertical integration. However, their having a great number of vessels, skilled management, an export-oriented and strong financial home base, made them one of the most profitable shipping firms in the world that are engaged in round-the-world services. The Corridor members had to deal with both highly integrated global firms and those with little integration though large number of physical assets and great mobilization power.

The above sources of global competitive advantage become impediments for the regional companies to become global. These impediments are represented by a number of obstacles i.e:

o smaller relative economies of scale in the national markets (Argentina / Brazil);

o limited geographical scope of the regional operators; limited availability of physical assets;

o lesser relative competitive financial schemes;

o outdated infrastructure and higher relative fixed and variable unit costs;

o more stringent central bank regulations;

o lesser developed cargo tracing or intranet systems;

o lesser relative ground transportation equipment versatility;

o lesser relative market intelligence operational capacity;

o lower relative productivity ratios in most logistic segments;

o high relative cargo volume concentration in a few locations;

o imbalanced trades;

o outdated or close to obsolescence assets: and

o a number of other more specific factors more inherent to the particulars of each logistic segment.

Porter (1985) argues that even when the advantages of global competition outweigh the impediments overall, the impediments can still yield viable strategic niches for national firms that do not compete globally. This was the case of Buenos Aires-based Maruba Shipping Company and Sao Paulo-based Di Gregorio, who let a global shipping firm (Haifa-based Zim Israel 
Navigation Company Ltd) enter into their market in exchange for them gaining access to a Zim's determined extra-regional market. More precisely, the Israeli firm joined the two South American companies in the east coast of South America whereas they were given access in exchange to the Middle East region - where they would have never been able to sail by their own means.

By doing this, the two lesser relatively developed and more regionally focused firms not only were given direct access to a new potential region, but also have benefited from absorbing a more advanced managerial, operational, and technological platform by giving up a relatively small market share to the global partner. This association later turned into a more formal joint-venture discussed in a later chapter and was replicated by many other global and regional companies.

It can be argued that this to be a win-win situation for those who preferred entering into some sort of value chain interaction with the global players instead of confronting them or looking for value chain mutations at a regional level. This win-win situation can be observed from a perspective that the global firm brought a valuable knowledge component along with higher relative technology and physical assets whose impact was gradually transferred to the regional companies. Additionally, such inputs were utilized not only in the regional markets but also in the overseas markets they were given access to. The global firm not only enjoyed direct access to the South America east Coast Market, but also benefited from learning the regional modalities further to counting on a future ally in their always needed coastal feeding services in the markets the regional firms were let in.

It becomes apparent that there is not only one lane towards achieving competitive advantage when looking into the Corridor as a value chain, but that many different value chain interactions can be possible and were in fact undertaken both within the Corridor and beyond the Corridor. Many regional firms made up the Corridor while some saw an opportunity to interact with the global players by setting up joint-ventures and alliances of various types. Others simply opted to sell out and form part of the global companies' horizontal integration strategy.

Thus the above illustrations highlight the complexity of the Corridor's evolving role as a CoP and means for both economic and its business transformation role can be seen to deepen. Clearly, 
while this example (the Corridor) of a complex program or work is potentially overwhelming as a subject for a doctoral research project, it has elements that can be examined as exemplars of the concept and scope limitations that prevent more detailed discussion and investigation may be forgiven as being a function of the scope of a doctoral study. The context of the Corridor best allows extensive study by multiple researchers over an extended timeframe not available to this researcher.

The coming chapters will examine the way knowledge sharing and transfer, cooperation and collaboration, and value chain interactions all combined to help both the regional and global firms enlarge economies of scale and gain competitive advantage through several cases where the Corridor was pivotal to these ends.

\section{5 - CHAPTER SUMMARY}

Several key concepts become apparent from this chapter. Firstly, the globalization process has provoked a situation where most of the firms that were earlier considered rivals have become united under the Corridor's roundtable forums across the region. The Corridor's role can be seen in this light as an integrating project to link firms in various forms of relationships and collaborative associations.

The real magnitude of the global threat was overwhelming for these firms and therefore brought about an adequate space where these companies could get to know each other and let the germ of knowledge sharing spread out in a way like never before. Naturally, face to face interaction seemed to be a key element in making these companies start sharing tacit knowledge, whereas a developing technological platform proved to be an adequate enhancer to knowledge transfer. This process enabled a growing number of players to deem the Corridor as a suitable arena to acquire knowledge by letting knowledge sharing and transfer effectively flow among its participants. Thus, the Corridor's role can also be seen as a knowledge marketplace or project to connect CoPs

It is clear that this situation though quite innovative, was not born in the regional companies’ intimate desire to become more efficient per se. But on the contrary, it was driven by the very need of facing a tsunami of global players who saw themselves to a different extent somehow 
compelled to also expand their market coverage and enlarge their economies of scale to remain competitive at global level. Not having done so would have meant for them loosing global positioning and therefore jeopardizing global leadership. At this point, the Corridor's capability to serve as a knowledge marketplace through its regional roundtables suggests that it proved useful for the regional firms in helping them formulate and implement a number of defensive strategies where knowledge sharing and transfer was the very first and key step. This chapter helped explain the unique contextual factors that triggered this condition.

Secondly, knowledge dissemination among the many regional members would not have been feasible without a genuine spirit of corporate cooperation and individual collaboration within the Corridor. This entity seems to have succeeded in helping the regional players to contact with one another and quickly adapt to a new business context. This adaptation process meant that the regional firms could comprehend how to face much larger corporations showing a high degree of vertical integration, an enormous home base competitive advantage, a global approach to strategy, and a relentless improvement, innovation and change attitude on the part of the global firms. Thus, gaining competitive advantage through cooperation and collaboration at the roundtables became a supporting tool for knowledge transfer to take place.

Thirdly and perhaps most importantly, the Corridor has enabled the formation of a number of joint-ventures and alliances among the members as well as between members and global players. These vertical and integration processes not only helped the members rationalize their assets and engage in innovative forms of cooperation, but also brought value to them in terms of access to new technology and updated managerial techniques. The context of the Corridor can be seen as being a complex change management agent and the various initiatives that took place under its auspices can be seen as both change management process projects and transformational projects.

Figure 4.2 illustrates how the Corridor along with its regional roundtables indicate that it played a vital role in the adequate channeling of knowledge sharing and transfer first, to later become a truly enabler of cooperation and collaboration through its members (and even non-members), to finally let the companies' value chain interact and gain competitive advantage in a number of different ways. 


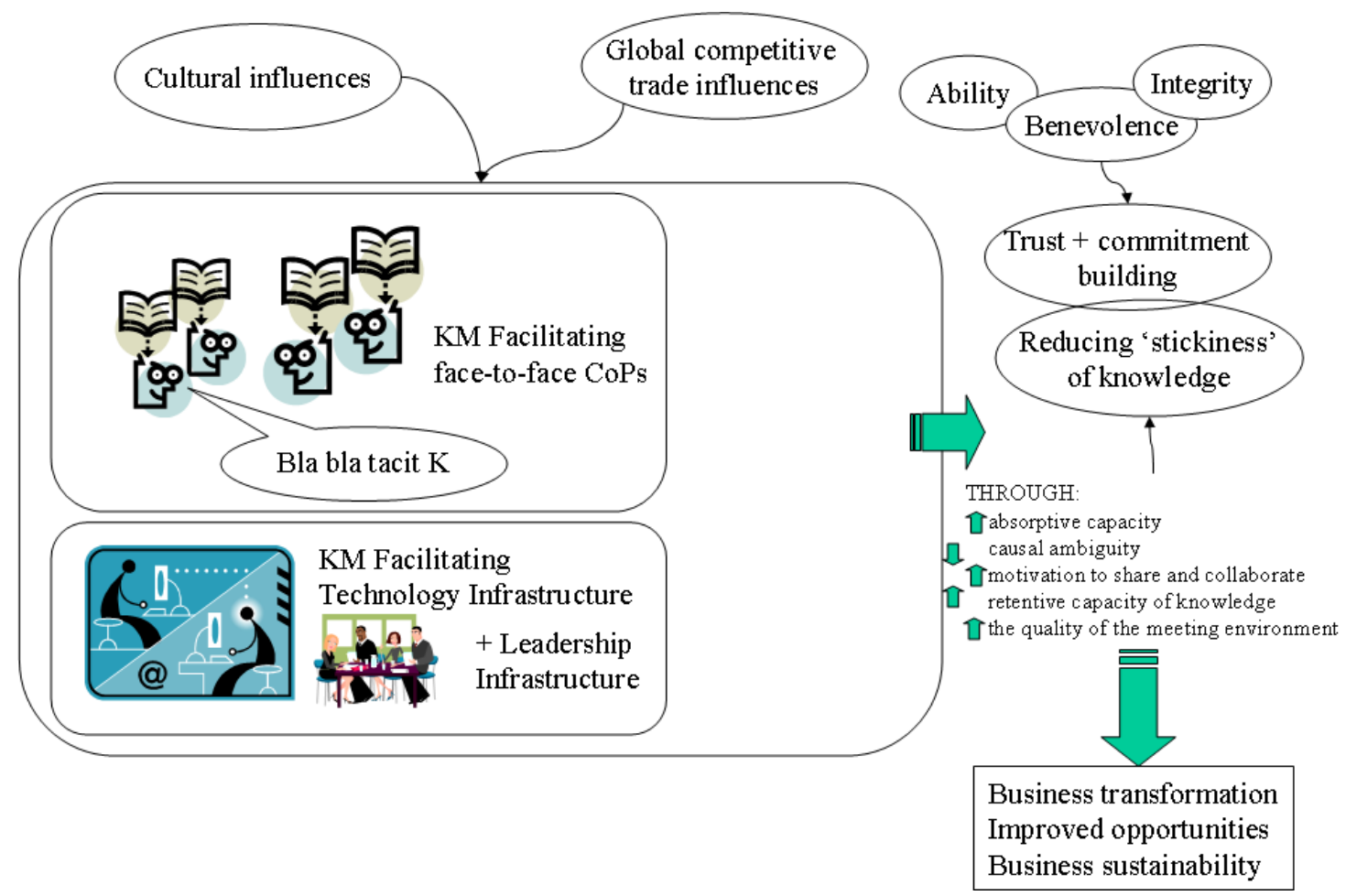

Figure 4.2: Summary of the Role of the Corridor in response to the shock of global competition and being shaped by the Region's culture, history and geography.

The key and core element is the roundtables that formed effective CoPs that stressed face-to-face interaction but was facilitated by leadership to design and enable this project to occur in the first place and to be supported by technology. The allowed the knowledge creation, reframing and transfer process to happen that also reduced knowledge stickiness and at the same time built trust between partners as confidence increased in the ability and integrity of participants that collaborated and the face-to-face interactions helped to develop a sense of benevolence. This helped bring about business transformations, improved opportunities and business sustainability. Example will be provided of these in following chapters.

The aim of this chapter was to introduce some aspects that provide a strong contextual background and highlights potential area of focus. In light of this, the case studies that will be investigated and reported up can only scratch the surface of this vast program of projects. They will provide useful and key analysis of how knowledge creation and exchange was undertaken, how alliances or collaborative arrangements were facilitated and how this impacted upon the likely competitive advantage of those firms involved. 


\section{DISSERTATIONS, PROPOSITIONS, AND RESEARCH METHODOLOGY}

\section{5 .1- INTRODUCTION}

This chapter defines the dissertation's propositions, study aims, and research questions. It establishes the reason why the Corridor provides an important research subject in terms of a PMrelated thesis topic. The research questions are discussed in detail while leading to a comparative analysis using a qualitative research approach. While it examines the pros and cons of both quantitative and qualitative methodology approaches, a multi-case study type of approach is developed and proposed as the most suitable method to study a project of this type. Different aspects of a case study research methodology are also examined along with a special section dedicated to sources of evidence and validation. Conclusions of the chapter follow.

\section{2 - RESEARCH PROPOSITION, STUDY AIMS, AND RESEARCH QUESTIONS}

Further to the concepts previously referred to in chapter 1 and based on the research idea, aims, and objectives, the research proposition is that the MERCOSUR Atlantic Corridor Project has become an instrumental entity, where regional stakeholders find a useful system and the adequate tools to formulate and implement a business reengineering strategy towards achieving larger economies of scale and gain competitive advantage. As such, the system entails stakeholders engaging in a journey of organizational culture transformation to further adapt to a new business context that was perhaps simply unthinkable in the recent past. The globalization trend that brought about the establishment of the Corridor also required for the stakeholders to adopt very difficult decisions as to whether to reengineer, integrate, downsize, or enter into some sort of joint-venture or alliance model aiming at overcoming trans-national operational deficiencies and gaining regional competitive advantage - all of it embodying both a leadership and knowledge management dimension whose potentiality remained till then apparently hidden or perhaps unnoticed.

The essence of this study is to establish how a project-focused organization, the Atlantic Corridor, from a huge geographical region along with a growing number of participants from that organization re-engineered their business activities. This entails overcoming regional and often 
even national cultural misconceptions which act as significant barriers to integration or obstacles toward achieving larger economies of scale and for participants to gain competitive advantage. Evidence will be provided to indicate the degree of success achieved by Corridor participants in this transformation endeavor. The choice of business transformation was cited by Artto and Kujala (2008) as an important PM research area that has been under-researched.

The globalization process along with its immediate response represented by the Corridor, introduces research possibilities allowing for various research questions to be raised relating to business transformation. These offer the chance to investigate a number of business transformation cases of very different nature that can be considered to be a program of transformation projects. Consequently, a number of research questions naturally present themselves in connection with the proposition and the embodied aims and objectives of the dissertation as proposed in Section 1.5:

- $\quad$ Research Question 1:

What are the variables granting the Corridor project the ability to generate trust first and facilitate knowledge transfer among stakeholders?

- $\quad$ Research Question 2:

How do Corridor stakeholders share vital internal information and join forces to face extraregional threats?

- $\quad$ Research Question 3:

Why do Corridor stakeholders tend to adjust themselves and adopt new attitudes and motivations toward committing themselves with partnerships, alliances, and joint-ventures?

- $\quad$ Research Question 4:

How does culture play a role in easing or impeding the development of the Corridor vision throughout the region?

- $\quad$ Research Question 5:

What factors hold a more relative influence on stakeholders to allow vertical or horizontal integration to occur?

- $\quad$ Research Question 6: 
What is the actual awareness degree among stakeholders with respect to sustainable development and its future relevance over the region?

\section{3 - QUANTITATIVE AND QUALITATIVE RESEARCH METHODS}

Positivism is based on the idea that facts exist in reality and that are subject to being measured. A reality may entail not only a single dimension of "truth" but various dimensions and therefore needs a measurement tool based on multiple variables. Under this perspective, the more variables that are added to a study the closer a researcher is believed to come to capturing an objective reality. A positivist approach is more likely to be associated with quantitative methods of data collection and analysis through statistical methods. Dunford (2004) introduces an alternative approach to research by arguing that the social situations and interactions require different research responses than those embedded in a given objective reality. The actions of people individually and collectively - are based on their constructions of the nature of the world in which they operate. Thus, the meanings people attribute to situations shaping an interpretative process, determine the actions that they take. This approach is known as interpretivism and entails a more subjective dimension and in-depth focus on data that is more likely to be associated with a qualitative methodology based on a small number of cases rather than on largescale survey methods.

\subsubsection{Quantitative Research}

Utilization of appropriate measures such as those suggested by Oliver (1997; pp284-5) in the form of scales, may give a number of options that vary according to the event or variables to be measured. None of the scales turn out useful if considered as technical tools to collect data. A scale to measure stability of attribution is of no use in capturing the different realities and numerous perspectives arising out of so many interactions between companies, activity segments, and subjective views leading to a wide array of different reactions. A categorical scale - even if designed to comprise a wide spectrum of situations - seems inappropriate to register in-depth comments, views, and subtleties that often become associated with corporate events. An ordinal scale does not help much either in detecting small and subtle changes, nuances or new perspectives in a response. Both the Corridor and the globalization phenomena can be considered 
as dynamic processes in permanent evolution and therefore cannot be ideally contained or summarized in a quantitative scale-type method.

Burton and Steane (2004; p41) contend that if a scale is very restricted, one will not be able to identify differences between those holding lots of experience and others who have very little experience. In terms of this research study, a scale showing too little discrimination can make firms, individuals, and particular situations look similar when actually they are not. National and regional companies representing different segments of activity within the Corridor may have distinct views and perspectives that turn out highly unlikely to be accurately reflected in a scale no matter of what type. However, even though a more discriminating scale showing more categories might help to better classify a firm's different angles or opinions, the rather high rate of personnel rotation among the companies operating in the region can make such scales of little relative value. With this high churn rate of employment, executives tend to adopt a cautious attitude toward expressing what their real views and opinions are with respect to a given business context or adopted strategy while holding a new position - not only with respect to their new companies but also their former firms they continue to keep ties with. This is especially true when an extremely changing or threatening business scenario tends to exert an increasing level of pressure on the participants to appear to remain loyal to former corporations and individuals. So, quantitative research methods involving responses to questions using various forms of scales introduce recognized limitations.

Under a quantitative perspective, data can be collected using a variety of methods, such as surveys (e.g. satisfaction scores), direct measurement (e.g. score on a test) and historical records (e.g. share prices over time) (Burton \& Steane 2004; p144). Choice of the modeling technique (by which a different emphasis is laid on the number of surveys to be sent out, the probable response rate to the questions included in the survey, what the incentives are for people to respond and what its variability is) determines how sensitive the survey design turns out to be in relation to the way that data are gathered.

The above, however, does not quite fit into what the Corridor may generate in terms of data. This is because it is an entity that is made up of a significant number of cases (each of them unique in 
many aspects) that go through processes that may look at first sight conceptually similar but in fact generate data in unique ways. One might speculate that every logistic segment might well share or gather a similar typology of data. However, reality shows that every case is the result of very particular circumstances inherent to the firm's strategic positioning in the region at a given moment. Further, the prime motivation of these organizations was survival in one of many ways or to sell the business in face of what was almost universally regarded as an economic "tsunami". The generation of data in a common form across Corridor participants was not a high priority. Thus, the research context does not favor standard quantitative data gathering techniques.

A brief sample of conditioning variables that suggest that a quantitative survey type of data collection would not be the most appropriate is provided as follows:

- Complementation of distinct segments leading to various types of integration;

- Different economies of scale and therefore cost structures;

- Distant locations and a diverse cultural spectrum all spread over a huge geographical area;

- An heterogeneous list of business assets to be optimized along with a tangible lack of infrastructure in some markets; and

- National barriers represented by associated cultural prejudices and operational obstacles.

To conduct a survey holding with a valid response rate turns out to be unrealistic in a Corridorlike organization within South America. This likely outcome may be explained as being derived from both a limiting cultural factor leading to a general distrust for surveys of all kinds, and an ever critical business context where no time is left for reflective learning or strategic thinking on the part of the Corridor members. The latter being a direct consequence of the pressure exerted by the speed and scale of globalization's impact. As such it assures a very low number of respondents - no matter how well designed and user-friendly a given survey turns out to be.

Research methodology literature often recommends that researchers should draw up a polite and informative covering letter explaining to people a number of good reasons why they should fill in the survey and how this may contribute to society in general. However, it has been my experience 
that the urgency of the immediate always prevails over any other strategic or research endeavor. Even to offer the respondents a copy of the results and to make it clear that the survey is of an academic nature and not a commercial one, cannot be reasonably expected to pave the road towards reaching the objective of a good response rate. Skepticism and lack of genuine interest and time appear to normally prevail.

My direct and deep experience of the context of this research suggests that research methods literature that favors quantitative research approaches may have been written possibly for societies with less survival-critical, urgent or more stable business contexts. Cultural asymmetries and economic contexts present in less developed regions such as that under study here, shape potential respondents' attitudes towards investing their time to respond to surveys and so the priorities of this group of people tends not to support academic quantitative research. As will be stressed recurrently throughout this dissertation, people from South America tend to privilege a short term tactical view at the expense of what might become a long term and more strategic vision yielding welfare in the long run. The immediateness of the urgent (recurrent transformations) prevails over issues of more strategic profile (potential growth) and therefore studies or surveys are definitely not a data collection technique to rely on.

\subsection{2 - Qualitative Research Methods}

Shank (2002) defines qualitative research as the study of processes and behaviors in their natural settings, through which the researcher tries to make sense of phenomena and the meanings that people attribute to them. Denzin \& Lincoln (2000; pp1-28) argue that qualitative researchers often use multiple methods to tease out the complexity, depth and richness of the natural setting being studied. In principle, the concepts of complexity, depth, and richness seem to match the needs of the Corridor from a conceptual approach to research methodology.

Research methods can include observation, conversation, interviewing, and participative or archival research. I was part of the Corridor project management team and so that brings two key elements that ease data collection with an in-depth view arising out of my direct engagement in intense, prolonged, and direct exposure to the field. I played the role of both a project's participant and direct observer. This form of engagement serves the researcher objective of 
capturing perceptions and understanding the actors "from the inside" so as to better comprehend how they make sense of and manage their daily activities (Miles \& Huberman 1994).

Further to the value of reflective practice being a core element of this research, as supported by the work of Raelin (2007), I was able to reflect upon my role as Corridor facilitating agent and as a Corridor participant. Coghlan and Brannick (2005) support the use of what they term participatory action research (PAR) in which the research is an active and participating member of events being studied. They argue that this is valid because the participating action researcher brings deep insights, large measures of tacit knowledge and often shares substantial trust and social capital assets with those being researched in such situations. The also note that PAR can be subject to bias so that researchers should always be mindful of this potential bias and take active measures to minimize any bias.

Unlike the quantitative research approach that relies more on analytical statistic models, the qualitative researcher's analysis is based on a careful and deep understanding of language and idiomatic turns, gestures meaning double sense responses, body language, ambiguity, as well as the often existing thin differences in concept and argument that become evident during an informal conversation or casual meeting. It seems that a qualitative research methodology fits better into a model that needs comprising diversity, difference, and uniqueness. This is because the Corridor involves many everyday routine processes (e.g. roundtable forums / discussion panels / cost structure assessments) as well as exceptional or unusual events, situations or practices (e.g. vertical and horizontal integrations / joint-ventures / VSA) that provide opportunities for deep observation and authentication of data.

Qualitative research also permits researchers to apply multiple theoretical perspectives as part of a strategy of accumulating understanding of the complex world in which we live, and allowing research and research findings to reflect many differing and changing social and organizational cultures, histories and contexts (Flick 2002). The Corridor can be categorized as a defensive reaction arising out of a complex extra-regional phenomenon (globalization). Consequently and further to containing a rather great diversity of situations and individual realities of unlikely standardization, it seems to adequately favor some form of qualitative research approach. 
A qualitative research methodology may comprise a number of potential alternatives:

* Historical methodology: even though this method has made great contributions to understand the present by explaining the past, it is not intended for the Corridor in view of its recent or contemporary nature beyond a brief explanation of the Corridor's historical and geopolitical context.

* Ethnography: it focuses on describing and interpreting a group of actors and their cultural systems, its roots lying in cultural anthropology studies of primitive cultures by early twentieth-century anthropologists (Creswell 1998; Glesne 1999; Silverman 2000). The Corridor being a contemporary event made up of both individuals and corporations falls outside the scope of this methodology.

* Phenomenology: it is concerned with how people make sense of their everyday activities and how they develop meanings from their social interactions. It focuses on actors' descriptions and interpretations of their lived experiences in relation to a particular phenomenon that the researcher is investigating - they way they are experienced and perceived (Creswell 1998; Glesne 1999; Gubrium and Holstein 2000). Prior experiences, knowledge and views should be set aside by the researcher to refocus exclusively on the data gathered through extended interviews with actors. The researcher conveys to the reader a sense of the essence of the experience; its underlying structure and what it was like to experience that phenomenon (Creswell 1998; Shank 2002).

* Hermeneutics: Burton and Steane (2004) explain it as the person reading a text about a subject, or holding a conversation or interview on an event or interaction, is just as involved in constructing a view of reality as the author of the text. In view it requires the researcher to draw on the historical context as the setting for their interpretation (Llewellyn 1993) - in order to reconstruct the past and anticipate the future - logistically it was too difficult for one researcher to perform ethnographic studies on all players/communities/tribes.

* Field-based case study: the researcher is the actor himself (or one of the actors inserted in an event) studying phenomena in their natural context over a period of time as a form of action research (McNiff and Whitehead 2000) in which the research participated as described earlier. Although better developed in the upcoming section, field researchers can 
be outlined as those who focus on actual events, activities, processes, people and relationships to generate outcomes embodying rich descriptions and analyses of contexts and practices (Adler and Adler 1994; Ferreira and Merchant 1992; Neumann 2003; Silverman 2000). Data from these studies can be made sense of by using grounded theory approaches (Glasner and Strauss 1967) in which themes are explored and developed to derive meaning and explanation.”

Field-based case studies produce theories that explain observations in specific contexts by embodying a cultural dimension and interpreting contemporary management practices in a given socio-economic context. Therefore, it is been chosen as the most suitable research methodology for the Corridor project. It turns out suitable for the researcher to gather subjective data and develop specific contextualized theories that explain actual observations made in the field (Dawson 1997; Scapens 1990). Following this rationale, grounded theory is also a component of the chosen research methodology in that a number of research questions about a phenomenon have been posed in order to inductively generate relevant theoretical elements through data collection and analysis (Glaser and Strauss, 1967).

\section{4 - CASE STUDY RESEARCH}

The flexibility that case studies allow for multiple research approaches/tools to be used to investigate a phenomena with, turns out convenient for a Corridor-type of project. A case study approach proves useful in presenting detailed accounts of organizational practices, in scanning the cultural perceptions as a channel to a better understanding of the different actors, and in interpreting management practices in their socio-economic, institutional and organizational contexts (Silverman 1985; Walker 1985; Werner and Schoepfle 1987). Potential research methods may range from semi-structured to quite highly structured processes. These methods include observation of activities, processes and meetings, unstructured and semi-structured interviews, and documentary and archival research (Creswell 1998; Miles and Huberman 1994).

In view of the many existing different realities embodied in the various logistic segments that make up the Corridor along with its roundtable forums, a case study approach allows the researcher to design the interviews in a variety of forms instead of imposing a rigid format. 
Structured questions in a close-ended format are less likely to be responded to, and even uncomfortable to be asked, as the nature of the interviews and the occasions in which they were held were highly informal. Thus, semi-structured (open and closed-ended questions) or unstructured (open-ended questions), or even a combination of those turned out to be easier to formulate and more likely to be accepted and duly responded during the many face-to-face occasions throughout the roundtable forums, lunches, breakfasts and events.

The business context in which these interactions took place was often quite critical and therefore the mood of the actors were far from optimal to be approached with a kind of questionnaire or formal approach whatsoever. These companies were struggling to survive and usually going through tough reengineering processes; some were just assessing whether to join forces or simply sell out and disappear, while others facing a potential threat of bankruptcy. Consequently, informal encounters and relaxed conversations in a neutral setting was found by the researcher to be the best strategy to gather personal views and perspectives which otherwise would have been unlikely to be truly reflected in a close-ended question type approach.

The dynamic nature of the Corridor as a mother project embodying a number of sub-case studies may well be associated with Winter and Checkland's (2004) view, in that to "manage" anything in everyday life is to intend to cope with an ever-changing flux of interacting events and ideas that is continuously unfolding through time. They further argue that the manager tries to improve situations that are seen as problematical and the job is never done because as the situation evolves, new aspects calling for attention emerge, and yesterday's solution may now be regarded as today's problems - a constant that becomes apparent in a recurrent way through various examples on horizontal and vertical integration that are better captured in a case-study format or approach. Winter and Thomas (2007) argue that the typical or traditional approach to project management practice tends to be rather narrow or limited in that it focuses on planning, organizing, coordinating, and controlling, but do not fully reflect organizational reality as messy, ambiguous, fragmented, and political in character. All these aspects can be better captured and assessed in a multiple-case study approach within a business context governed by change and uncertainty. 
The Corridor project study is primarily exploratory, where representative individual case studies of business transformations help to describe the workings of the Corridor by explaining its unique particularities. Findings are used to explain how these transformations were achieved.

Even though each strategy has its distinctive characteristics, there are large areas of overlap among them (Sieber 1973). Yin (1994 p15) distinguishes at least five different applications for case studies as far as evaluation research is concerned. They tend to explain the causal links in real-life interventions that are too complex for the survey or experimental strategies; they describe an intervention and the context in which they occur; they can illustrate certain topics within an evaluation in a descriptive mode; they serve to explore those situations where the intervention being evaluated does not lead to a clear and single set of outcomes; they may be a meta-evaluation or a study of an evaluation study (Smith 1990; Stake 1986).

The MERCOSUR Atlantic Corridor case study project is developed here as the major focus of study of and analysis as a single case (main) study though it embodies study of a number of representative short term sub-case studies and a larger and more detailed long term sub-case study. These sub-case studies help strengthening the units of analysis and address more consistently the research questions within a pattern-matching dimension by incorporating different angles and conclusions arising out of a greater number of situations. The thesis design therefore looks more like a multiple-case design dissertation despite containing the three basic elements of a single-case design: critical test; unique case; revelatory case (Yin 1994, p45).

Yin contends by definition, that the unusual or rare case, the critical case, and the revelatory case constitute the basic components of justification of a single case. He further argues that the rationale for single-case designs usually cannot be satisfied by multiple cases. However, and as it will be exposed in the upcoming chapters, the various short-termed cases and the larger longtermed case are all aimed at addressing every research question from different logistic segments, particular angles, and specific perspectives. All of them will turn out useful means to demonstrate how different actors displaying great resource base asymmetries can reengineer and produce change towards enlarging economies of scale and gaining competitive advantage - two concepts that are inclusive of a knowledge, cooperation, trust, ethics, and cultural dimensions. 
The Corridor project can be regarded as a mother project embodying a significant number of multiple-case studies without which it would turn out to be a hollow concept with no substance. The very sub-case studies are precisely the project's backbone that allows the project itself to grow and consolidate even further across time. The sub-case studies do also interact among themselves leading to a more fluent knowledge sharing and transfer avenue that make no other thing than enriching and constantly feeding the cycle. The evidence from multiple cases is often considered more compelling, and the overall study is therefore regarded as being more robust (Herriot \& Firestone, 1983).

The idea behind of conducting a multiple-case study design is to be able to reach cross-case conclusions. Each individual case study consists of a whole study, in which convergent evidence is sought regarding the facts and conclusions for the case. Thus, each case's conclusions are then considered to be the information needing replication by other individual cases. Another aspect that is of paramount importance and that is dealt with in the coming section is given by both the sources of evidence and the validation process.

\section{5 - SOURCES OF EVIDENCE AND VALIDATION}

Yin (1994 pp 80-90) refers to six major sources of evidence from case studies along with its strengths and weaknesses: documentation; archival records; interviews; direct observations; participant observation; physical artifacts. All these sources with the exception of the latterwhich is mostly utilized in other type of research such as anthropology - are far from being isolated pieces of evidence for the Corridor project but that on the contrary, they turn out to be highly complementary.

The available documents attached to this dissertation include a number of roundtable forum's minutes which clearly describe the nature of the problems discussed in the gatherings as well as the number and diversity of participants along with their main concerns. An author's conference paper (Arroyo and Walker, 2004) presented at the University of Otago, Dunedin, New Zealand, on the Corridor project's contribution to knowledge management also helps substantiate the content of the dissertation. A second paper presented at EURAM Conference in Liverpool, 
England, on the Corridor's contribution to business transformation (Arroyo and Walker, 2009) also helps conceptualizing its actual regional role. Also, a co-authored book on this very project recently published in Australia also corroborates the fundamentals of this thesis, its main contents having been exposed to the public scrutiny of both academics and practitioners (Arroyo and Walker, 2008).

The archival records are made available in the form of maps, charts, and graphs comprising the vast region the Corridor project embraces, as well as the names of participating firms and the typical commodities that give birth or sustain the very nature of a given problematic situation. These sources of evidence, however, cannot be deemed as very relevant or of extreme importance but that they serve as a good complement to help the reader better comprehend or conceptualize the essence of the problems that are intended to make clear as well as the various potential ways to work them out.

Interviews are of fundamental importance as they bring testimony of a wide array of situations which tend to repeat across the various case studies and therefore become useful for replication purposes. They were designed and implemented in two different ways. Firstly, in an open-ended nature and mostly informal, spontaneous or accidental fashion they were administered by the author who intimately knew respondents and had their trust. Those interviewed were informed of the aim, purpose and intent of the interview and all applicable documentation relating to this was administered as required by the RMIT ethics approval process. These interviews took place at sporadic times between 02/1996 and 12/2005. Validation of data was ensured by clarification with interviewees of content at the time of questions being asked and subsequently through further conversations, emails and other documented forms. Thus, file notes, diary entries and post hoc confirmation documents provided authenticated valid data to work with.

Secondly, in a more formal or structured pattern of more general evidence emerged from a questionnaire that had been distributed by a consulting company member to the Corridor that had received a rather high rate of response. The Corridor project had already commenced before this study began and so the motivation for Corridor participants was high and their need to supply valid data to gain reliable feedback was also strong. Further, this data was not gathered by me and 
therefore I did not seek or receive any ethics clearance from RMIT, however, it did form documented quantitative evidence that was useful as background information. This Corridor sanctioned type of survey and its informational-value response les in stark contrast to any further attempts to gain additional non-Corridor sanctioned quantitative survey data. Both the said quantitative methods survey data and qualitative data gathered in this research proved useful and complementary to further accomplish another step in the validation process. Even though the interview strategy adopted later for this research was intended to produce a number of critical responses from a limited number of key representative respondents to open-ended questions, it was the more formal or structured questionnaire that guided the trend and nature of the overall replies. Key interviewees tended to concur in general terms with the outcomes and findings of the formal questionnaire although their comments and views turned out to be richer and more detailed. Interviews are often only regarded as verbal reports and therefore subject to bias, poor recall or even inaccurate articulation, however, the previous questionnaire's outcome turned out to be a solid tool to corroborate interview data with information from other sources. When coupled with documentary evidence such as minutes of roundtable meetings, the authenticated evidence form interviews and observations provided powerful and rich triangulation of evidence.

Both my direct and participant observation were critical to understanding the many complex variables embodied within this project, despite the potential bias an internal observer or participant is often argued to hold. The major problems related to participant-observation have to do with the potential biases produced (Becker 1958). However, being a participant of the project's development from its initial stage has helped the author better design and target the various questions in an open-ended fashion, as well as better conceptualize responses and gestures about a complex project which otherwise would have been unlikely for an external-type observer who was not familiar with the project itself nor with a deep understanding of its modus operandi and philosophy. Raelin (2007) also advocates the value of the kinds of epistemology of practice that reflective practitioners bring to bear on action based learning. In a sense, this work is also a product of a massive action learning exercise undertook by all Corridor participants as they engaged in learning activities that they struggled to make sense of. The nature of the Corridor experience was, as this thesis will demonstrate, one of learning and so not only were participants 
engaged in action learning but the author's role as facilitator and intermediary in critical events reported upon in this thesis also followed an action learning trajectory.

The following paragraphs support the way that co-learning, which could also be seen as a form of action learning by participants as suggested by Raelin (2007), also provided validation through action, reflection, and further action cycles. It must be appreciated that all participants are experts in their field and so this forms another level of validation. Cicmil (2006 pp27-37) addresses in a critical and constructive manner the nature of knowledge, skills and competencies that are relevant to practicing project managers in their everyday coping with the complexity of contemporary projects. The critique concentrates on the functionalist/positivist goal of disseminating best practice in project management to large number of practitioners under a kind of commodification of knowledge across management fields by focusing on planning, organizing, coordinating and controlling without reflecting the often complex project's realities as messy, ambiguous, fragmented, and political in character. Cicmil and Hodgson (2005) argue that the widely accepted conventional project management knowledge system tends to reduce or marginalize complex and subtle political and social processes to simplistic "critical factors" or problem areas that arguably can be removed through an appropriate action.

Cicmil (2006) further contends that an interpretative qualitative methodology itself can present the researcher with a significant challenge as it involves much more uncertainty and ambiguity than traditional positivist research, and demand a high level of communicative skills, richness of one's vocabulary, sensitivity to moral and ethical issues, and a wide theoretical repertoire. The Corridor project can be defined as both uncertain and ambiguous in itself in view of the many different existing realities and the apparently ever evolving or mutating situations of very difficult anticipation. Cicmil (2006) stresses that this methodology also requires an enormous amount of both enthusiasm and personal discipline, and a willingness to take risks that inevitably come with interrelating with people in a research situation which has a participative character. In fact, my participation began at the outset of the project - which helped me understand much of what today would be almost impossible to conceptualize without having first interacted from inside - while today acting as an outside researcher - which makes biases even more unlikely to be produced or alternatively offset by having fulfilled both roles. 
Complex projects have been defined by Williams (2002 pp58-9) in terms of the dimensions of structural complexity and uncertainty. Structural complexity is derived from the interaction between the number of elements which make up the project and the interdependence of these elements. Uncertainty is derived from the lack of clarity and agreement concerning project goals and the means to achieve goals. The result is that project elements interact in complex and unpredictable ways and undermine attempts to manage them using a rationalistic project management framework.

Flyvbjerg (2001, p136) argues that the feel for the game is central to all human action of any complexity, and it enables an infinite number of moves to be made, adapted to the infinite number of possible situations, which no rule, however complex, can foresee. Schon (1983) contends that given the complexity of real-world situations, practitioners are not usually able to follow or apply the ready-made prescriptions and propositional knowledge taught on training programs. Real-world situations are always much more complex than the textbooks imply and therefore intuition and experience can make all the difference. These are the reasons why I considered it an advantage having my role as participant of the Corridor project at a given point and therefore a participant observer. Such a role enabled me to really interpret the many insights of a complex project which otherwise an outer observer would unlikely capture and interpret when observing it from a typical processual perspective.

Schon (1983) further contends that practicing managers constantly have to deal with complex situations for which there are no right answers while the way they deal with these situations is rarely through the systematic application of textbook theories, but through sophisticated processes of "reflection-in-action” (thinking on one’s feet), and reflection-on-action (thinking back on events and planning the next move). Having to look back to reflect on the past to better comprehend the current situation and interpret the "whys" of the next moves proved to be of outmost relevance for the author while the very complexity of the various situations meant in fact an effective barrier to develop any biases. 
Winter and Gale (2003) argue that the classical research dissertation is not always appropriate for busy managers on part-time programs because the academic activity of producing a research dissertation is somewhat far removed from everyday practice of managing. They further highlight that studying part-time and keeping focused on a specific field brings about an opportunity for mature students to develop their own experience of managing through a period of more deliberate and more reflective engagement in real-world practice. As Brown and Duguid (2000) state: "learning to be requires more than just information. It requires the ability to engage in the practice in question". Such an engagement in fact comes naturally when playing both observation roles: direct and participant observation.

In terms of the approach to the analysis of data to address the research questions and general aim of the thesis, a combination of a sensemaking and grounded theory approach was adopted. Weick has long been considered an authority on sensemaking and recently (Weick, Sutcliffe, and Obstfeld, 2005) linked its value with that of reflection from experiential learning. The state that "sensemaking involves turning circumstances into a situation that is comprehended explicitly in words and that serves as a springboard into action." they continue to say "The emerging picture is one of sensemaking as a process that is ongoing, instrumental, subtle, swift, social, and easily taken for granted. The seemingly transient nature of sensemaking (“a way station”) belies its central role in the determination of human behavior.” (Weick et al 2005, pp409). This illustrates how the data was treated along with developing themes and meanings through grounded theory.

Grounded theory has also been a long established approach to build theory from the ground up (Glaser and Strauss, 1967) and (Strauss and Corbin, 1998) using real data such as that described above. Meaning is developed from analysis of a range of data sources, bearing in mind and guarding against biases referred to earlier, by undertaking a conscious and sub-conscious process of coding the data and formulating and testing themes of findings against theory and accepting, adapting or building theory to address the research exercise.

\section{6 - CHAPTER SUMMARY}

After presenting the research questions first listed in Section 1.5, and discussing the philosophical stances, merits and usefulness of quantitative and qualitative research approaches, it remains 
clear at this point that given the nature of the Corridor project (uniqueness; size; multiple insights; trans-national configuration; trans-cultural in essence; multi-sector-oriented) a qualitative research approach turns out more inclusive and therefore appropriate than a quantitative method. A qualitative method enables the researcher to better cope with the many subtleties that are hidden or immersed in a number of situations arising out of the globalization process and its impact on the Corridor project. Furthermore, data availability along with the way it might be gathered suggests too that a qualitative approach proves more adequate and flexible to capture the various realities. Finally, the chapter concluded with a section clarifying the data sources used and the way that findings were developed and validated. 


\section{SUB-CASE STUDIES OR SUB-UNITS OF ANALYSIS}

\section{1 - INTRODUCTION}

This chapter presents four different and unique logistic case studies that illustrate and provide data relating to research questions that this dissertation is focused upon. Underlying circumstances and the evolving nature of global and regional coincidental actions refer to the way that distant and heterogeneous global players or firms can collaborate, exchange data and information, and cooperate with one another under the set of described particular circumstances.

The way that each case study's collaboration group responded to the globalizations process for each case study provides concepts that are developed and outlined to explain how these collaboration groups transformed their business approaches. A number of concepts connected with this thesis' contributions interact and are exposed and discussed to bring about a unique view of these case studies which provide answers to the research questions.

The chapter describes each of the four case studies and addresses key concepts that made possible both global and regional companies achieve larger economies of scale to gain competitive advantage. Each case study turns out to be very specific and unique with respect to its operations, strategy, and leadership style. However, each developed their strategy in a similar way that exposes a similar rationale governing issues of collaboration, cooperation, knowledge sharing and transfer, leadership, and culture.

The first case study features an ocean shipping companies’ joint venture (JV) linking organizations with a very different combination of structure, nationality, culture, technology, and vision. The JV comprised two regional firms and two extra-regional companies. Each of these firms followed very different strategies in the way they got together and how they agreed on the fundamental aim of facilitating outsiders to become regional operators and for them to become more international and global in future. The overarching aim from the outset was to avoid incurring heavy investments. 
This case study strongly relates to issues of the role of cooperation, leadership, knowledge, and corporate strategy in driving the business transformation.

The second case involves a multimodal transportation business transformation, through the way that it connected its operations in ports of the Mediterranean Sea in Europe to end destinations in South America's West Coast. More specifically, this project entailed the set-up of a land-bridge between west Mediterranean ports (Spain; France; Italy) and the industrial pole of the SantiagoValparaiso area through the port of Buenos Aires in Argentina by combining ocean, road, and rail transportation on a door delivery mode, that is to the customer's 'door'.

This case study will demonstrate the important role of successfully combining traditional knowledge, physical assets, and management to achieve differentiation and gain competitive advantage.

The third case study refers to the way the longest and most strategic railways in Argentina passed on vital knowledge about how foreign companies could reengineer themselves and become a competitive asset not only at regional level, but also in a global dimension. Without collaboration, cooperation, and leadership by partners in this case study, the business transformations would have not occurred.

This case study illustrates the logistic complementation of knowledge and leadership between the various agents involved that was pivotal in allowing the transformation project to successfully evolve.

The last case study embodies a Buenos Aires up-river operator that realized the urgent need to react to the globalization process by becoming regional and gaining world-class competitive advantage to face threats posed by emerging global maritime terminal operators' structure, technology, management, and investment. 
This provides a typical reactive model where two regional players enter in a JV to face global companies that might easily outperform any of them individually. Aspects of leadership, cooperation and collaboration, strategy and knowledge become apparent.

Each of the four case studies refers to short-term tactical transformation project actions. They are individually presented and developed and outcomes and insights from them are compared to provide findings summarized in the chapter's conclusions.

\section{2- CASE STUDY \# 1: INTRODUCTION}

This section is further complemented by Chapter 2 that sets the context of this study. Before the Corridor was born or even prior the globalization process became unquestionable in South America, the region was serviced by a limited number of shipping companies. Some were regionally-based with limited number of lines linking the region to overseas or regional markets, while just a few were extra-regional and displaying a more diversified commercial base, though none had reached the definition of global shipping company in the sense of operating around-theworld services or being engaged in global logistics.

At that time the concept of door-to-door transportation was already known and implemented across South America though there was practically no integration among the logistics services. Ocean transportation, port operations, customs clearance services, road transportation, rail transportation, fluvial transportation and air-freighting were all separate businesses that were carried out by independent companies showing little or no operational complementation. Forward or vertical integration strategies were by then just pure theory as was horizontal integration. At this point one might easily speculate that company owners or CEOs had no concept of the competition or market expansion that they would soon confront.

The region or the market was protected by cargo reservation laws which tended to favor those ships nationally registered and flying the national flag - provided that a genuine link existed between the port of registry and the ship's flag. The lack of integration trends by then suggests there was no clear or identifiable need to enter into this type of venture. This was due to the relative safety granted by protective laws as well as the inexistence of any level of innovation that 
could enhance or promote a minimum degree of competition. Porter (1980, p301) corroborates this particular situation well by arguing that the essence and decision of the vertical integration must go beyond an analysis of costs and investment requirements to consider the broader strategic issues of integration versus the view of a financial market transaction. The regional companies failed to consider these aspects which later flourished almost naturally within the Corridor and under a very different business environment.

The shipping business in South America was to a great extent cartelized and therefore the competition turned out to be negligible and business complementation was practically absent. Also, a general increase in operational costs was caused by increases in the shipping-related ancillary service costs combined with the shipping business's ageing fleet. Large national companies became larger, despite a tendency towards what would have normally created an unfavorable business scenario, and expanded overseas though without reaching the status of becoming global shipping companies such as Argentina's Elma with more than 45 vessels or Brazil's Lloyd Brasileiro with more than 50 vessels. More importantly, these large national companies failed to catch up with upcoming international trends of lower costs and global logistic reach. These companies grew up and became relatively global thanks to governmental subsidies duly backed by geopolitical visions of those who by then were in charge of the maritime interests within the shipping policies secretariat of both Brazil and Argentina.

Global carriers started to open their services in SAMEC ${ }^{56}$ first - where larger economies of scale became apparent - to later cope with SAMWC ${ }^{57}$ (a region that held strategic value for its proximity to Asia) while those foreign-based liners already servicing South America began diversifying their lines. They did so by adding new ports of call to their schedules, either by launching new lines or combining with other existing liners either through combined services or by making use of extra-regional transshipment ports to cover new and distant markets.

During this transformational and growth process, possibly made confident by the on-going restrictive cargo laws, the regional ship-owners did not react nor realize the by then emerging

\footnotetext{
${ }^{56}$ SAMEC: South America East Coast.

${ }^{57}$ SAMWC: South America West Coast.
} 
global trends of struggling to achieve larger economies of scale or diminish operational costs. Regional ship-owners remained indifferent to competitive newcomers and the trends they brought with them. By contrast, foreign-based lines with enough international experience to be accustomed to dealing with the international shipping free market foresaw what globalization would bring to South America and reacted by extending their services in an attempt to achieve larger economies of scale and remain competitive to face any upcoming market transformation. Gradually, national flag owners had to face not only foreign-based vessels holding a much greater carrying capacity and a much lower slot cost, but also saw their cargo reservation laws being eroding and having less and less impact on securing them a minimum market share that enabled them operate without investing and therefore surviving. This process took place at the same time, and following a similar path in both Brazil and Argentina with the other countries of the region remaining consistent with the two bigger neighbors. To better illustrate the relative size and structure of the companies that make up this case study it becomes necessary to briefly assess each of them in the next section.

\subsection{1. -Structure of the joint-venture companies}

Sources of data and information gathered for this first case and used for analysis of this study from the JV companies can be summarized as follows:

Table 6.1- Sources of Data for Case Study \#1

\begin{tabular}{|l|l|l|l|}
\hline Company & \multicolumn{1}{|c|}{ Source } & \multicolumn{1}{|c|}{ Content } & \multicolumn{1}{|c|}{ Notes } \\
\hline Zim Lines & $\begin{array}{l}\text { General context } \\
\text { information }\end{array}$ & $\begin{array}{l}\text { Data Gathered on } \\
\text { present services and } \\
\text { global structure from } \\
\text { corporate website. }\end{array}$ & Data gathered in 2008 (www.zim.co.il) \\
\hline Hanjin & $\begin{array}{l}\text { General context } \\
\text { information }\end{array}$ & $\begin{array}{l}\text { Data gathered on } \\
\text { present services and } \\
\text { global structure from } \\
\text { corporate website. }\end{array}$ & $\begin{array}{l}\text { Data gathered in 2008 } \\
\text { (www.hanjin.com) }\end{array}$ \\
\hline Maruba & $\begin{array}{l}\text { General context } \\
\text { information }\end{array}$ & $\begin{array}{l}\text { Data gathered on } \\
\text { present services and } \\
\text { global structure from } \\
\text { corporate website. }\end{array}$ & $\begin{array}{l}\text { Data gathered in 2008 } \\
\text { (www.maruba.com.ar) }\end{array}$ \\
\hline Zim Lines & $\begin{array}{l}\text { Operations } \\
\text { specific } \\
\text { information }\end{array}$ & $\begin{array}{l}\text { Business strategy and } \\
\text { the impact of the } \\
\text { upcoming trends on } \\
\text { both the region and } \\
\text { the regional shipping } \\
\text { market. }\end{array}$ & $\begin{array}{l}\text { Regular short, informal and frank } \\
\text { conversations normally held during } \\
\text { lunch time and working periods between } \\
\text { 03/1996 and 12/00. Concepts arising out } \\
\text { of these conversations were written } \\
\text { down and discussed over and over from } \\
\text { time to time in view we were colleagues }\end{array}$ \\
\hline
\end{tabular}




\begin{tabular}{|c|c|c|c|}
\hline & $\begin{array}{l}\text { Operations } \\
\text { Manger for } 6 \\
\text { years at Delta } \\
\text { Line; } \\
\text { Multimodal } \\
\text { Manager for } 10 \\
\text { years at Elma. }\end{array}$ & & $\begin{array}{l}\text { working for the same company. Content } \\
\text { was checked with the source and } \\
\text { validated during } 2007 \text {. }\end{array}$ \\
\hline Multicargo & $\begin{array}{l}\text { CEO for South } \\
\text { America for } \\
\text { more than } 10 \\
\text { years; Country } \\
\text { Manager for } \\
\text { more than } 15 \\
\text { years at } \mathrm{K}+\mathrm{N} \text {, in } \\
\text { charge of ocean, } \\
\text { ground, and air } \\
\text { transportation } \\
\text { departments. }\end{array}$ & $\begin{array}{l}\text { Business strategy to } \\
\text { take the best possible } \\
\text { out of the situation in } \\
\text { terms of market } \\
\text { intelligence as well as } \\
\text { knowledge sharing. }\end{array}$ & $\begin{array}{l}\text { Several meetings, both private and } \\
\text { business, where concepts and trends } \\
\text { were reviewed and opinions exchanged } \\
\text { on an informal fashion throughout 1997, } \\
\text { 98, and 99. I have been in regular contact } \\
\text { and had the opportunity to discuss in } \\
\text { detail various aspects of the potential } \\
\text { contribution of the Corridor, in view of } \\
\text { my position at the Corridor and } \\
\text { Multicargo being a regular customer of } \\
\text { Zim. Content was checked with the } \\
\text { source and validated during } 2007 \text {. }\end{array}$ \\
\hline Maruba & $\begin{array}{l}\text { Operations } \\
\text { Manager for the } \\
\text { River Plate for } \\
\text { more than } 12 \\
\text { years and an } \\
\text { extensive } \\
\text { experience in } \\
\text { marketing and } \\
\text { multimodal } \\
\text { business across } \\
\text { the region. }\end{array}$ & $\begin{array}{l}\text { Business development } \\
\text { strategy connected } \\
\text { with both the Corridor } \\
\text { as an enhancer and the } \\
\text { JV as an enabler to } \\
\text { intend becoming a } \\
\text { global operator. }\end{array}$ & $\begin{array}{l}\text { Regular discussions and exchange of } \\
\text { opinions during regular business } \\
\text { meetings, joint trips, and lunch time on } \\
\text { numerous occasions. Extensive } \\
\text { discussions were involved from } 1996 \text { up } \\
\text { to 2000, period in which notes were } \\
\text { taken and images of situations and } \\
\text { events were kept fresh in my memory. } \\
\text { Content was checked with the source and } \\
\text { validated during } 2007\end{array}$ \\
\hline Di Gregorio & $\begin{array}{l}\text { Brazil marketing } \\
\text { manager for } \\
\text { more than } 20 \\
\text { years. }\end{array}$ & $\begin{array}{l}\text { Leader-follower views } \\
\text { and cultural } \\
\text { differences within a } \\
\text { Corridor roundtable } \\
\text { forums context. }\end{array}$ & $\begin{array}{l}\text { Isolated comments and informal } \\
\text { statements gathered during JV meetings, } \\
\text { coffee breaks, and dinners while } \\
\text { traveling together around South } \\
\text { America. Some comments were written } \\
\text { down while others just kept in mind as } \\
\text { they resulted to be obvious or common } \\
\text { to the on-going business context. } \\
\text { Content was checked with the source and } \\
\text { validated during } 2007 \text {. }\end{array}$ \\
\hline
\end{tabular}

The JV was made up of four shipping companies of different size, service scope, technology and management. Two of them were foreign-based ${ }^{58}$ out of which only one ${ }^{59}$ was already operating in SAMEC. The other two were from Argentina and Brazil though displaying very different structure and strategies: Buenos Aires-based Maruba was a traditional Argentine flag shipping company that was successful under the protective cargo reservation laws during the 1970s and 1980s. Rio do Janeiro-based Di Gregorio was a successful and well established road

\footnotetext{
${ }^{58}$ Zim Lines of Haifa, Israel and HANJIN SHIPPING of Pusan, South Korea.

${ }^{59}$ Zim Lines.
} 
transportation firm in Brazil with extensive services all over the country that wanted to become a ship-operating company as an extension of their well established on-shore operations aimed at securing cargo flows for their on-shore operations and creating synergies between the latter's customer base and their needs to be serviced overseas.

Even though identifying regional firms with even moderate degree of vertical or horizontal integration was by then very rare, these two companies entered into a substantial degree of integration. This possibly may explain why they also entered later into the maritime JV. Maruba also operated tug services in the port of Buenos Aires further to owning a limited number of trucks with which exerted some control on part of their cargoes. Further to that, they also reached a moderate level of internationalization in that they operated a number of shipping lines linking SAMEC with the North Atlantic market, the Asia-Pacific region, SAMWC, and the PNW ${ }^{60}$ region. In this particular case one might conclude that even though it was a company that had already launched itself to the international arena, there were many steps yet to be taken for it to truly become a truly global player - both in terms of geographical scope and the extent of its trans-national value chain.

Di Gregorio owned a fleet of over 600 trucks to service Brazil first and Argentina later, but also expanded their activities into warehousing and cold-storage facilities. The operation of the latter ones might well be considered as horizontal integration, although it can also be regarded as natural assets for a trucking company to carry out their services with a minimum physical backup. This company was by then regarded as within the top $10^{61}$ road transportation firms within the MERCOSUR region with an extensive regional market coverage and significant distribution assets spread all over the coastal cities and inland destinations, which might explain the above mentioned facilities as a natural settings to sustain the firm's expansion rather than strategic moves towards accomplishing an objective of greater dimension.

\footnotetext{
${ }^{60}$ PNW refers to the Pacific Northwest Region that embodies North America's west coast.

${ }^{61}$ Di Gregorio counted on distribution facilities in Sao Paulo, Santos, Paranagua, Sao Francisco do Sul, Rio Grande, Porto Alegre, Rio do Janeiro, Vitoria, Curitiba, Salvador, Recife, Fortaleza, Belem, and Manaos - further to a number of hub warehouses placed in strategically located inland cities where neither railway or barging services were available. Through a network of agents they also had access to key locations within Paraguay, Uruguay, Bolivia, and Argentina.
} 
This company based its corporate growth on Brazil's economic dynamism which showed a consecutive growth and development for a number of years ${ }^{62}$. This might well indicate at this point that this firm wanted to exert some control on the incoming cargos' routing decision making by committing to the launching of new maritime services. However, I had the opportunity to address these issues with the president of both companies who repeatedly confirmed that this kind of integration was not intended nor viewed as a strategic dimension by the time it was adopted. The decision to collaborate evolved purely from a tactical financial transaction opportunity costs basis that appeared to be timely when made. As they repeatedly said, "we are not in a position to keep our assets under-utilized for a long time but that we have to join our structures to make the business sustainable”.

Even though these two companies could well be regarded as the exception and not the rule (in view of their incipient integration degrees at the outset of the globalization process) they never undertook their value chain take-over acquisitions with the intention of catching up with upcoming trends - their decisions were merely triggered by timely situations, absent from extraregional strategic thinking. It also turns out interesting to consider different structures and management styles of the bigger global firms involved in this JV because these global players more consciously took a similar strategic path. The following outlines some relevant features of these global firms taken from their web sites:

a) Hanjin Shipping ${ }^{63}$ : is Korea's largest carrier that operates some 60 liner and tramper services around the globe transporting over 100 million tons of cargo annually. Its fleet consists of some 200 containerships, bulk and LNG carriers. A member of the Hanjin Group, Hanjin Shipping has several subsidiaries including Keoyang Shipping and Senator Lines GmbH, and affiliates including logistics IT specialist CyberLogitec, Pyeongtaek Container Terminal Co., Ltd., ship management company, Hanjin SM (Ship Management), and terminal operation company, HPC (Hanjin Pacific). Hanjin Shipping has a comprehensive global business network with 3 regional headquarters, 200 overseas branch offices, and 30 local corporations, earning about $90 \%$ of its total revenue overseas. Hanjin Shipping's world-class logistics network includes 11 dedicated

\footnotetext{
${ }^{62}$ Brazil's GDP showed an average growth of $2 \%$ during the 80 s and above $3 \%$ during the 90 s while the trade between Brazil and Argentina expanded more than proportionately during said periods of time.

${ }^{63}$ Information available at www.hanjin.com
} 
terminals in Long Beach, Tokyo, Kaohsiung, and Busan among others and six inland logistic bases in such locations as Shanghai, Qingdao, and Port Kelang. 2 more dedicated container terminals are scheduled to open in the near future; one in Busan New Port of the development Phase 2-1 in 2009 and the other in Jacksonville, Florida in 2011. Furthermore, Hanjin Shipping's ship repair yard in China scheduled for completion in 2008 will provide efficient repair services not only for its own vessels but also for other carriers, thereby functioning as a new source of income for the company. In early 2003, Hanjin Shipping allied with COSCO of China, Yang Ming of Taiwan, K-Line of Japan, and Senator Line of Germany to form 'CKYHS Alliance,' the world's largest strategic alliance in this industry sector. The CKYHS Alliance has enabled Hanjin Shipping to sharpen its competitive edge by broadening its service coverage, offering Express Services $^{64}$ and sharing space with the allies to lower costs. To achieve its goal of becoming "the premier total logistics service provider respected by the global community, In addition, Hanjin counts on a number of 3PL ${ }^{65}$ businesses on track and building a ship-repair yard as part of its business diversification efforts.

b) Zim Lines ${ }^{66}$ : has more than 60 weekly services with rapid transit times to 180 ports of call end-to-end multi-modal services reaching destinations on five continents, full-service logistics, including state-of-the-art hubs in Asia, the Americas and Europe, and regional offices throughout the world: four operational headquarters, an extensive regional office network and agents throughout the world.

To reduce the total supply chain costs, Zim has created the ZPL Global Logistics Network with hubs in China, the Caribbean and Europe. These world-class logistics centers offer a full range of services such as freight forwarding, customs brokerage, consolidation and deconsolidation, offdock container terminals ${ }^{67}$, warehousing, trucking and container repair, liner and feeder services connecting Asia, Europe, Africa, North and South America, and the Middle East; end-to-end

\footnotetext{
${ }^{64}$ Express Services are those linking two ports straight with no transshipment or on-carriage services.

${ }^{65}$ The term 3PL refers to is a firm that provides outsourced or "third party" logistics services to companies for part, or sometimes all of their supply chain management function. Third party logistics providers typically specialize in integrated warehousing and transportation services that can be scaled and customized to customer's needs based on market conditions and the demands and delivery service requirements for their products and materials.

${ }^{66}$ Information available at www.zim.co.il

${ }^{67}$ Off-dock terminals are those facilities located outside the ports' primary zones where operations of various kinds can be conducted.
} 
services; and a multimodal network for end-to-end shipments to thousands of inland destinations on six continents, with unique strength in China.

It has local support with extensive customer and logistical services with three well-located hubs. Zim currently operates a fleet of about 100 vessels, deploying a total carrying capacity of over 240,000 TEUs. This includes 13 state-of- the-art Panamx 4,900 TEU vessels added in 2004. Zim offers 60 services to ports of call throughout Europe, the United States and Canada, Central and South America, Africa and Asia. They have established a particularly strong presence on routes between the Far East and the Caribbean, Mediterranean and China.

It becomes apparent from the company profiles outlined above, that both firms rely upon global structures along with a considerable degree of both horizontal and vertical integration. On the one hand there are shipping companies, shipyards, marine terminals, and logistic facilities that form part of the global firms' integration strategy where South America fell totally or partly out of their operations radar. South America appears to be somewhat of a gap in the integrated chain of logistics that is more commonplace elsewhere in the world for these global players-this presented a challenge and a gap to be filled by these organizations in a bid to be truly global.

On the other hand, an opportunity for forming an alliance or JV presents itself with a regional company such as Maruba that showed more daring than the other JV partner - Di Gregorio - in terms of pretending to compete at an international level despite showing both serious weaknesses in those areas where the global firms had undisputable strengths (larger carrying capacity / faster vessels / bigger load factors / lesser consumption / more efficient loading and discharging rates / well functioning JVs around the globe / superior technology). The next section describes and analyses the way these four companies got in touch with one another and evolved until effectively agreeing on entering into a joint-venture on a win-win basis.

\subsection{2.- The corridor as a facilitator}

The situation that unfolded can be illustrated by Figure 6.1. 


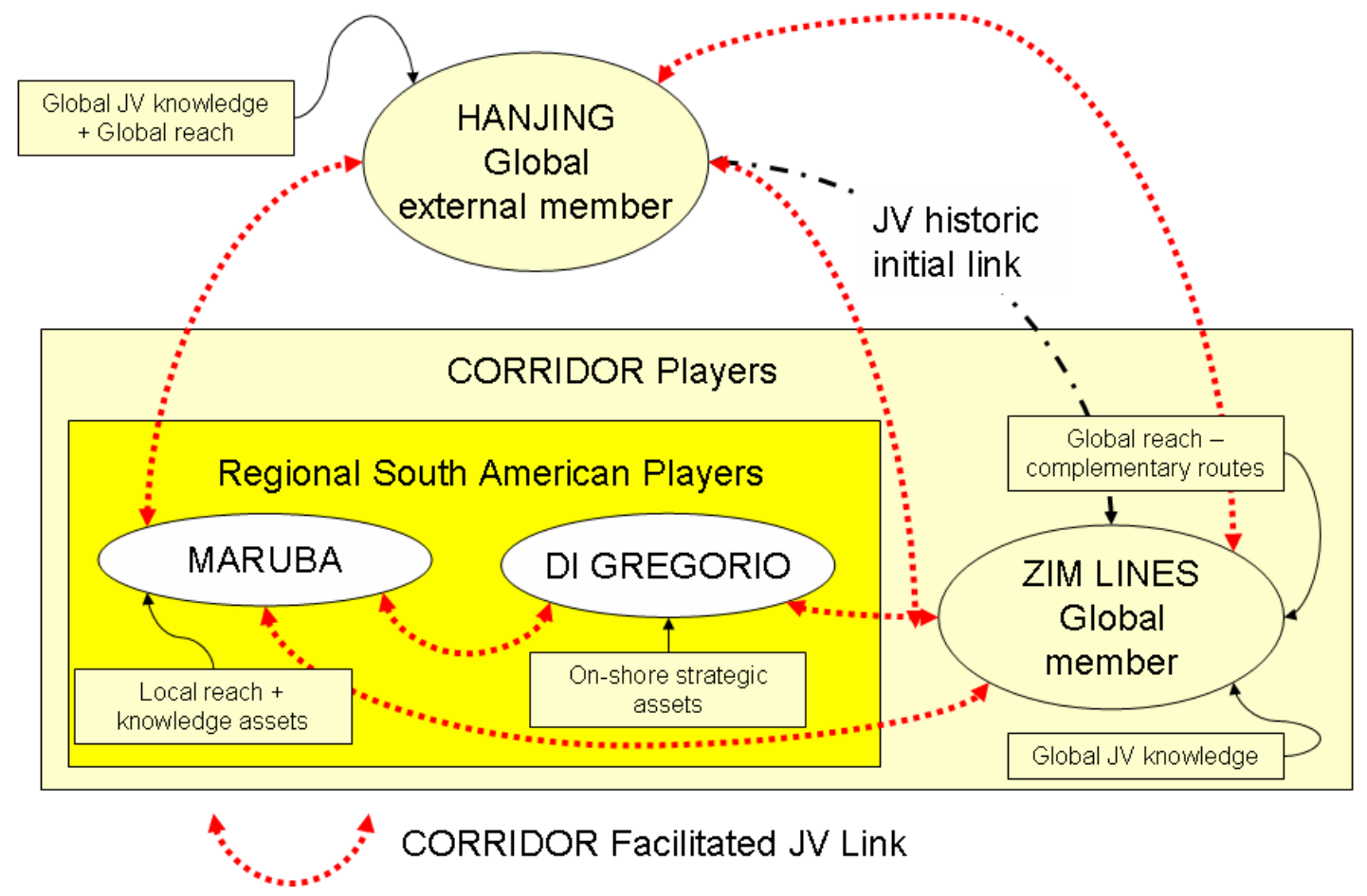

Figure 6.1: Joint Venture Formation Scenario to Gain Win-Win Outcomes from a Rationalization and Effective Asset Re-Alignment Project

Not only were Maruba and Di Gregorio original members of the Corridor but also Zim Lines had well established roots in the region when globalization pressures approached South America. Even though Hanjin was an outsider as it did not service South America, however it had entered into a JV with Zim Lines in different trading areas of the world where the market scales were substantially larger and competition much better developed than in the MERCOSUR. Therefore Hanjin and Zim operated under the same concepts and philosophy with which many JVs, alliances, and partnerships of various types were evolving elsewhere and these matured with the passing of time and as the globalization impact reached closer to the MERCOSUR.

One retrospective view of events suggests that Zim Lines’ executives, who already had experience from their Asia-Pacific's alliances, proposed the germ of the idea that advantages of combining physical resources could be brought into the Corridor roundtable discussions. At this point, there was probably a kind of pre-corridor knowledge sharing and transfer process evolving 
from different global players who subsequently joined the Corridor with business experience of such scenarios and these were eventually introduced to the Corridor.

I suggest that the following is a realistic representation of how the concepts and arguments inherent to putting into practice cooperation and collaboration (combining various resources, initially knowledge sharing, with knowledge transfer introduced later) through discussions with various Corridor and access to other more tangible records of accounts that I have obtained from Corridor players and assembled from my own reflections. These were the pillars of a structure that let these companies to undertake a reengineering process within the Corridor.

My reasoning and analysis for this follows the strategic position each player held at the time of forming the JV:

Zim had on service 12 vessels spread over three different lines with:

o The Mediterranean service ${ }^{68}$ sailing every 10 days (with 4 ships);

o USEC $+\mathrm{G}^{69}$ service (with 4 ships); and

o $\mathrm{USWC}^{70}$ service (with 4 ships), both based upon weekly sailings.

Northbound and southbound vessels utilized a hub port in Kingston, Jamaica. This hub was a centre for directing all kind of operations to reach practically any destination in central and south America, the Caribbean, continental US and Canada, and further combinations toward extraregional ports in the Asia-Pacific region through the Panama Canal. Eastbound and westbound vessels utilized a hub port in Barcelona, Spain from which a long list of ports and inland destinations were accessible along West and North Africa, Eastern Europe, near, middle, and Far East - in addition to the traditional destinations of Western Europe.

Maruba had a similar number of vessels in service out of which only 3 were assigned to their Mediterranean service and this entailed a sailing every 20 to 25 days. Therefore it was one of Zim's competitors in the Mediterranean market. This company also had in service a number of vessels in lines that were out of the scope of interest of the other JV

\footnotetext{
${ }^{68}$ Mediterranean services include all those ports that lie on the Mediterranean sea from Israel up to Spain.

${ }^{69}$ USEC+G means United States East Coast and Gulf. Even though it refers to a service to and from the USA, it also includes Canada's Atlantic ports.

${ }^{70}$ USWC means United States West Coast. Even though it refers to a service to and from the USA, it also includes Canada's west coast ports.
} 
members at the time talks were held. Their operational hub port was Montevideo, Uruguay where a fully containerized service to Patagonian ports was operated based on a monthly sailing. These services were to SAMWC, SAMEC, PNW, and the Asia-Pacific region. None of these services were in principle of any strategic interest to the parties. However, at a later stage - as it will be explained later in this chapter - it became subject to negotiation in their search to gain competitive advantage and extend their geographical scope even further. Both tugboat services and trucking operations were also carried out in the Buenos Aires Province that also provided a given degree of vertical integration.

Di Gregorio had neither vessels of their own nor any chartered. In fact it had no maritime background at all. However, it did have a significant on-shore cargo-volume out of which $75 \%$ was export or import-oriented. This was their main motivation to become involved in a maritime venture that could eventually grant them a given level of control on both the incoming and outgoing cargo flows. It counted on a number of on-shore hubs or inland nodes spread all over Brazil where it conducted all sorts of warehousing and distribution operations to and from the MERCOSUR partners. This company had operations from practically all Brazilian ports and their fleet units were versatile enough as to be capable to carry both container and over-dimension cargoes.

$>$ Hanjin: South America was a pending issue for this shipping company even though it was a huge global shipping company with world class logistic facilities and an impressive degree of both vertical and horizontal integration. It was engaged in a large number of trade lanes, either by operating its own vessels and lines or through JVs and VSA with other shipping companies as large as them. It had enormous inland transportation and port operations capabilities across the globe. Also it deploying the latest maritime technology in terms of effectiveness which meant that by the time it approached the region it possessed enormous economies of scale and therefore the lowest slot cost, automatically leading to gaining competitive advantage. In doing so, it becomes apparent that Hanjin also needed a number of suitable partners in order to deploy its services efficiently with the required number of vessels and it needed sailing frequency to avoid oversupplying the market if it had decided to operate entirely by itself_-resulting in flooding shipping traffic with excessive tonnage. Such an action would have almost automatically made ocean freight rates fall dramatically because the MERCOSUR trade did not at that time, have the 
absorbing capacity for new operators of such an operational volume. This issue was salient to newcomers to the region as they took due care because it was in nobody's interest to destabilize the market by deploying an excess of supply - or a huge over-tonnage situation that would have eroded the freight level to unsustainable levels.

As a general concept, and as I will explain in detail later on, the JV's strongest point was given by having the existing ship-owners rationalizing their fleets (Zim / Maruba) without having to entirely flagging out a significant number of vessels of their respective fleets, but by partchartering their vessels to those who they were sure were not going to bring additional tonnage to the market (Hanjin / Di Gregorio). This process also entailed an orderly process of replacing older and bigger tonnage by newer fuel-efficient smaller vessels.

Thus, what before turned out to be two independent shipping companies each operating a number of vessels holding relatively low load factors, increasing operational costs, and a limited geographical scope, found through the JV, the way toward rationalizing their non-revenue generating assets without having to face any onerous exit costs.

Additionally and incidentally, these very same companies profited from one another in that they gained access to new markets, deployed better technology, developed global and regional management skills through knowledge exchange, and achieved larger economies of scale. These all led to JV partners gaining a new dimension of competitive advantage that could help them face new global trends in a more sustainable way.

The next section will address the advantages for each member with respect to the various operational stages this JV went through. Given the different structure and dimension of each company, it turns out interesting to identify how every company has found the way to build a win-win situation despite the existing heterogeneity.

Perhaps one of the greatest merits of the MERCOSUR Atlantic Corridor resides in having created the necessary ambience to let knowledge spread out among their members, build trust through 
cooperation and collaboration - all leading to undertaking a number of projects under a very special or unique regional business context.

\subsection{3. - J/V Project operational advantages}

It is essential for the reader to understand that the MERCOSUR had by nature unbalanced trade partners that varied according to the rate of exchange at any given time. Large regional country imbalances between imports and exports produced a serious container use situation for the shipping lines. Additionally, regional-world import and exports volumes also produced similar problems. Moreover, there were similar macroeconomic imbalances between MERCOSUR countries whose policies rarely coincided and therefore resulted in non-harmonized patterns of respective currency parities. This caused intra-regional trade imbalances that had to be offset or compensated for extra-regionally.

Typically, a devalued currency would foster exports and discourage imports while systematically promoting import substitution schemes. A revalued currency encouraged imports to grow more rapidly than exports thus producing trade imbalances-unless corrective policies were designed and put into practice to achieve higher industrial productivity. Whatever the case, being regionally or individually incurred, these trade imbalances often had a profound impact on all shipping companies with tight business operations in the region and little shipping activity abroad.

All loaded import containers that were brought into the region had to be re-exported empty or somehow subsidized to capture a lower existing volume of exports to diminish the impact of a negative balance - this known in the industry as "imbalance". The same situation was incurred when a devalued currency resulted in much bigger exports than imports. This caused a different trade imbalance because traded containers had to be repositioned in South America either empty or at relative lower ocean freight rates adjusted to the existing demand at any given moment.

One of the most worrying operational issues for a shipping company to manage is precisely the efficient management of hundreds of thousands of containers spread out all over the world. Most shipping companies held global leasing contracts with the global container leasing firms which 
let them hire and deliver huge numbers of containers around the world. It is with an inadequate supply of these containers, positioned in the right port at the right time, which prevents the shipping company from generating income. This may well result in a financial burden of holding and moving non-performing containers which can easily bankrupt shipping companies.

Most shipping companies hold global leasing contracts with the global container leasing firms which let them hire and deliver huge number of containers around the world. These containers allow a global shipping concept to work on a door-to-door basis, no matter what the origin or the destination may turn out to be. This concept is essential as it demands an accurate administration and operational follow-up for shipping companies to remain competitive. Consequently, the more globally or geographically operational a shipping firm becomes, the better chances it has to combine different balancing situations across the globe. Balances and imbalances may be better and faster offset or worked out by a shipping line that operates multiple trades and manage different demands, seasons, and operational limitations of various kinds. A company that is too committed with a single trade or region that has modest opportunities to efficiently work out the existing trade imbalances (e.g. MERCOSUR), is likely to consequently suffer inefficiencies by being not capable of maintaining high load factors and an effective rotation ratio of their container fleet.

Two further aspects are worth further scrutiny to better conceptualize the reason why the globalization wave reached South America and triggered a structural shipping change throughout the region. Firstly, global shipping lines engaged in the so called "Round-The-World" services (east-west-east) engaged in a continuous pursuit of achieving larger and larger economies of scale. As vessels grew larger and larger in their container carrying capacity, the ports of call tended to reduce in number and adopted the function of not only ports of loading and discharge aimed at servicing the country where they laid, but also ports or hubs where huge number of containers were transshipped to worldwide destinations.

Thus, vessels that used to serve in those trades became obsolete because of their lower relative carrying capacity and were therefore relayed to service markets not actually served by the roundthe-world services. Regions or markets became hidden or outside the radar of the ever-expanding 
shipping companies, all of a sudden it became in their interest to operate in emerging opportunities. South America therefore faced a wave of larger, more efficient, better equipped, and better managed shipping companies with the objective of entering this market. Many entered into JVs and alliances to avoid flagging in tonnage on top of the existing tonnage oversupply. Others overlooked this situation and deployed their entire fleets and on-shore facilities to service their global accounts the way they did it anywhere in the world. Naturally, this situation led to an even more delicate balancing situation as well as depressing the ocean freight level.

Secondly, the main economies of the MERCOSUR found themselves in the process of opening their economies to the world and reengineering a number of state-owned companies as well as generally modernizing their industries. This political process caught the attention of existing companies who foresaw a regional trade expansion business opportunity and acted accordingly. Many shipping companies that were traditional operators in the region started to improve their sailing frequency by flagging in new ships, positioning a more diversified fleet of containers, and calling at new ports. This led to a significant market oversupply so these companies faced the option of restructuring or disappearing. Several aspects are worth discussing in further detail:

$>$ Economies of combined operations: existing volume of cargoes (export and import) did not allow outsiders to deploy entire fleets and enlarge the carrying capacity to a level that would have turned out detrimental to both local and foreign operators so the shipping companies had to start talking to one another to find suitable combinations without harming the market. By the time the MERCOSUR Atlantic Corridor was established, the existing foreign trade ratio for Argentina was a 3 to 1 import ratio while Brazil had a more favorable 2 to 1 export ratio. Consequently while Argentina could be regarded as an import-oriented market holding a currency parity of 1 to 1 PESO/USD, Brazil turned out to be the opposite by having a parity of 2.5 to 1 REAL/USD parity. The Brazilian import ratio was more oriented toward reindustrialization of its economy than to consumption of goods as was the Argentine case. Brazil being a much larger economy and much more export-oriented than its neighbor Argentina caused the region to run out of containers for export, as there was an overall shortage of containers to supply the maritime export demand. Intra-trade between Brazil and Argentina was relatively balanced which meant that the excess of containers laying in Buenos Aires had to be repositioned in various 
Brazilian ports (Rio Grande / Itajai / Sao Francisco do Sul / Santos / Rio do Janeiro / Vitoria / Recife). Therefore and from a balancing standpoint, the more globalized companies (Hanjin / Zim) were useful to the regional ones (Maruba / Di Gregorio) in coping with an increasing imbalance problem to the advantage of all carriers. Imbalances in the Asia-Pacific market could be compensated for with an existing relative higher freight rate market, shorter transit times, better turn-around-time (TAT) ratios, or access to niche markets. For the bigger companies, facing imbalances this effectively turned out to be much easier in view of all their global opportunities to offset their imbalances in one way or the other and through their internal operational accounting systems. Regional firms agreed to flag-out a number of ships while the global firms flagged-in an equal number of vessels. This helped the former rationalize their operations effectively and the latter enter into a new market without bringing tonnage on top of what the market might tolerate (Hanjin), while also rationalizing by flagging out two of their existing vessels (Zim) on the Mediterranean traffic. The overall result was: a similar number of deployed ships; a well orchestrated rationalization scheme; a kind of joint administration; a betterment of the service based on management and technology; and a larger number of operators. Zim's hub ports in Kingston and Barcelona were utilized by Maruba and Di Gregorio where they could offer their customer base new destinations to reach; they also took advantage of the global firms' lower costs to carry out operations at the various terminals, hubs, and inland transportation means far from South America. In exchange, the global companies learned the way to access and do business successfully in the region from an operational, financial, managerial, and legal way. Hanjin also took advantage of Zim's hubs to connect with their round-the-world services while supplying the other members an immense spectrum of ports and inland destinations worldwide at much lower costs that any of the others would ever have access to. Hanjin's global transportation chain turned out to be of value not only for the regional firms but also for another global company like Zim that despite being global knew how to take advantage of Hanjin's larger scales, lower costs, and deeper integration status across the world. Combinations of all types involving ship-repairing, chartering and port operations became gradually apparent as the JV developed.

Economies of internal control and coordination: the cost of scheduling, coordinating operations (ships flagging in and out / bunkering / transshipment / balancing / crewing / 
dry-docking / money pooling / freight rate policy / financial policy) were set up in Sao Paulo, Brazil where Zim had their regional headquarters and information systems for the entire region. Having flagged out a couple of vessels within a pre-established rationalization program within the $\mathrm{JV}$, Zim proceeded to reduce their operations staff in Sao Paulo as well as Maruba who also flagged out two vessels and had their operational staff based there. Di Gregorio and Hanjin had to sign on new staff to fulfill a controlling function. Many operations were internally compared and only the most efficient and less expensive operation was jointly selected for members to permanently use. Ocean terminals, containers repair shops, chartering brokers in charge of supplying vessels, and all type of suppliers were rationalized and selected accordingly.

Economies of Information: integrated operations proved useful not only to reduce the need for collecting some types of information about the market, but also to jointly lower the cost of gaining information from a rather big number of dynamic variables related to the fleet's daily running cost (DRC) $)^{71}$. The operational centre in Sao Paulo, Brazil was also in charge of collecting, calculating, and distributing all the fixed and variable costs further to the setting of a margin which also embodied a permanent feedback from the marketing staff who were testing the market. Establishing individual carrying capacities according to the individual shares and looking into the evolution of both $\mathrm{T} / \mathrm{C}^{72}$ and $\mathrm{V} / \mathrm{C}^{73}$ rates was a function the coordination centre had to fulfill while taking decisions which deemed best for the interests of the members. Zim's intranet was made available to the other members for a fee which meant Zim reduced their operations centre cost structure while the other members also did so by dismantling their own regional systems and enjoying a more economic and efficient system.

Economies of avoiding the market: even though some internal transaction costs were faced by every member, marketing, selling, negotiating, and transaction costs of the market for the individual members could be significantly lowered or internally outsourced with the most efficient member. Maruba took a leading position in integrated logistics within the

\footnotetext{
${ }^{71}$ DRC: daily running cost of a vessel embodies all the cost variables that make up the running of a ship which once divided by 360 days results in a cost per day. It serves as basis to figure out slot cost, margins and prices or freight rates.

${ }^{72}$ T/C: time charter rates mean the on-going rates for hiring a ship on time basis at a given moment. It usually displays a daily amount in USD with which a potential charterer may consider for his calculations.

${ }^{73} \mathrm{~V} / \mathrm{C}$ : voyage charter rates mean the on-going rates for hiring a ship to sail from port A to port $\mathrm{B}$, where the charterer assumes the responsibility to afford all operational charges concerning the voyage/s.
} 
MERCOSUR region because of their knowledge and operational strengths. Maruba's commercial team undertook marketing and fixing deals for the group to share proportionally, instead of having every member engaged in any independent MERCOSUR-oriented sales. Maruba, Zim led the sales effort for the entire group to market the refrigerated cargo segment in view of their knowledge, connections with customers, and understanding of the market variables for the benefit of the group. Having to hire reefer cargo specialized professionals to work independently in every company would have resulted in an enormous waste of resources in terms of very high costs and loss of precious time to become known in the marketplace to cope with possibly the most yielding segment for a shipping company.

The variable costs table from a global shipping company illustrates the way the imbalance impacts on a given cost structure and the rationale behind it is presented in Table 6.2.

Table 6.2 - Shipping company’s variable costs structure for 20 foot containers in South America for Export (above) and Import (below).

\section{VARIABLE COSTS STRUCTURE FOR 20’ CONTAINERS}

\begin{tabular}{ccccccccc}
\multicolumn{10}{c}{ COSTOS EXPORTACION 20. DRY VAN (USD) } \\
POL & LOADING & FEEDING & DRAYAGE & BALANCE & EQPMT & COMM. & TOTAL \\
\hline BUE & 130 & - & - & -160 & 70 & $3,20 \%$ & 40 \\
MVD & 140 & - & - & -120 & 70 & $5,00 \%$ & 90 \\
ASU & 120 & 780 & - & -335 & 80 & $3,00 \%$ & 645 \\
RGB & 350 & - & - & 110 & 70 & $4,50 \%$ & 530 \\
ITJ & 300 & - & - & 70 & 70 & $6,50 \%$ & 440 \\
SNT & 345 & - & - & -200 & 100 & $6,50 \%$ & 245 \\
RIO & 400 & - & - & - & 70 & $6,50 \%$ & 470 \\
\hline
\end{tabular}

\begin{tabular}{cccccccc}
\multicolumn{1}{c}{ COSTOS IMPORTACION 20' DRY VAN (USD) } \\
POL & LOADING & FEEDING & DRAYAGE & BALANCE & EQPMT & COMM. & TOTAL \\
\hline BUE & 125 & - & - & 160 & 60 & $3,00 \%$ & 345 \\
MVD & 150 & - & - & 120 & 70 & $2,50 \%$ & 340 \\
ASU & 170 & - & - & -70 & 80 & $4,00 \%$ & 180 \\
RGB & 285 & - & - & 200 & 70 & $4,50 \%$ & 555 \\
ITJ & 450 & - & - & - & 80 & $4,50 \%$ & 530 \\
SNT & 290 & - & - & -110 & 70 & $4,00 \%$ & 250 \\
RIO & 120 & 750 & - & 335 & 70 & $3,00 \%$ & 1275 \\
\hline
\end{tabular}


The table headings have the following meaning: POL means port of loading along with the abbreviations of the most important ports of call on SAMEC; Loading refers to the cost of loading a container; Feeding is the cost of carrying a container either by feeder vessel or river barge which in this case is Asuncion in Paraguay (barging); Drayage refers to any ground transportation involved to and from any inland location; Balance is what it would cost for a shipping line to reposition a container from a low demand point to a high demand point; Equipment is the cost associated to the hire of the container; Commission is the amount of money a ship-owners pays to their agents for handling containers; and Total represents the final result of the logistic container cost.

The important aspect to bear in mind here is the balance column (5) where a number may be positive or negative according to a set of operational topics inherent to a particular port. Thus, both Buenos Aires and Montevideo on the river Plate show negative export figures and positive import figures. The rationale behind this is that the import freight rate already includes a balance cost which covers the repositioning cost of that container from the River Plate to another port in Brazil - provided that the import market keeps strong and avoided operators cutting rates to compete.

Conversely, the balance cost for the export shows negative values and therefore acts as tool to quote competitive ocean freight rates relating to intended evacuation of that container and avoiding incurring repositioning costs to Brazil, despite these being covered by the import freight. This entails the vessels holding unutilized space on board, or what in other words would mean an excess of supply at a given port at a given moment. Therefore it works as a kind of subsidy that aims at speeding up the TAT concept. The port of Asuncion in the landlocked country of Paraguay offers a different situation. Despite having more imports than exports, the former shows a negative balancing number while the latter shows a much bigger negative figure.

The rationale is that while the import trade to Paraguay is a very competitive one and therefore needs competitive freight rates, the excess of equipment is to a great extent repositioned at no cost on different locations along the Argentine side where there are plenty of exports. However, the situation may change the rationale if we address the 40 footers which are designed to carry 
voluminous and lighter cargoes. Table 6.3 shows the particular case of both Rio Grande (RGB) and Itajai (ITJ) ports in Brazil. These two ports have practically no imports and therefore show a neutral balancing cost on the southbound leg, while on the exports the balancing represents the re-positioning costs from nearby ports holding an excess of 40 footers (e.g. Buenos Aires; Montevideo). The port of Santos (SNT) faces both a high level of competition on the southbound leg and a growing need of containers on the northbound, this process leading to show a negative balancing on the import while keeping it neutral or competitive on the export.

Table 6.3: Shipping company's variable costs structure for 40 foot containers in South America.

\begin{tabular}{|c|c|c|c|c|c|c|c|}
\hline \multirow{2}{*}{\multicolumn{8}{|c|}{$\begin{array}{l}\text { VARIABLE COSTS STRUCTURE FOR 40’ CONTA } \\
\text { COSTOS EXPORTACION 40' DRY VAN (USD) }\end{array}$}} \\
\hline & & & & & & & \\
\hline POL & LOADING & FEEDING & DRAYAGE & BALANCE & EQPMT & coMM. & TOTAL \\
\hline BUE & 130 & - & - & -200 & 120 & $3,20 \%$ & 50 \\
\hline MVD & 140 & - & - & - & 120 & $5,00 \%$ & 260 \\
\hline ASU & 120 & 1350 & - & -485 & 140 & $3,00 \%$ & 1125 \\
\hline RGB & 450 & - & - & 150 & 120 & $4,50 \%$ & 720 \\
\hline ITJ & 350 & - & - & 100 & 120 & $6,50 \%$ & 570 \\
\hline SNT & 460 & - & - & - & 180 & $6,50 \%$ & 640 \\
\hline RIO & 500 & - & - & - & 140 & $6,50 \%$ & 640 \\
\hline \multicolumn{8}{|c|}{ COSTOS IMPORTACION 40, DRY VAN (USD) } \\
\hline POL & LOADING & FEEDING & DRAYAGE & BALANCE & EQPMT & coMM. & TOTAL \\
\hline BUE & 125 & - & - & 200 & 120 & $3,00 \%$ & 445 \\
\hline MVD & 200 & - & - & 120 & 120 & $2,50 \%$ & 440 \\
\hline ASU & 335 & - & - & -100 & 130 & $4,00 \%$ & 365 \\
\hline RGB & 400 & - & - & - & 120 & $4,50 \%$ & 520 \\
\hline ITJ & 380 & - & - & - & 200 & $4,50 \%$ & 580 \\
\hline SNT & 350 & - & - & -150 & 130 & $4,00 \%$ & 330 \\
\hline RIO & 175 & 1350 & - & 485 & 120 & $3,00 \%$ & 2130 \\
\hline
\end{tabular}

A different situation can be observed in Rio do Janeiro (RIO) where there is an historic overstocking of containers, this is because the import balancing cost is heavily charged while on the export side the balance variable is nil. While RIO is not often serviced southbound by the competition, practically all shipping companies are calling at RIO on the northbound leg of the 
journey. This is the reason why the Brazilian importer or foreign exporter can afford paying not only such a high balance costs but also the feeding costs via a third port. Both the frequency of sailings and the available carrying capacity define a given port terminal's competition level and consequently leads to a given balancing policy on the part of the shipping companies. Greater globalized or interconnected a shipping company leads to improved chances for optimizing shipping operations.

Every port has its own stock and balance position further to facing market particulars that are inherent to its own market dynamics and structure. Consequently it becomes apparent that counting on such a detailed matrix of information on a regular basis (not only for the region but also with respect to a long list of extra-regional ports that are somehow connected with the region on worldwide basis) entails a great effort in terms of market information, knowledge sharing and transfer, cooperation, collaboration, and definitely a good amount of trust. Whoever has a well organized and efficiently managed system of global equipment balancing already holds a strategic tool that will make its regional presence more sustainable than those who do not.

Result of a number of informal interviews that were conducted by the author during the JV and right after it started working will be exposed and compared with the author's own opinions and views based on his direct observation and participation throughout the process in the next section.

\subsection{4. - Interviews, comments and insights}

I played two roles relating to this specific case which gave me a parallel view and most importantly, the opportunity to informally discuss a variety of relevant issues to this case study with some of the referents at the time the JV was formed. Firstly, I participated in the negotiations on Zim's behalf as country manager for Argentina. Secondly, I initiated the promotion of the Corridor concept across the region by explaining it and recruiting potential candidates.

Therefore, in this dual role I was able to approach a number of key executives and gather, assess and validate their opinions, views, insights, as well as fears or contradictions relating to issues discussed. I was also aware of potential biases that could potentially arise out of my direct 
participation in one of the JV firms. This is why I laid a greater emphasis on discussing issues with Zim executives to offset personal insights that could excessively reinforce or support sought answers to the research questions.

It is my considered opinion that this JV owes its sentiment to: (a) false expectations of the region's growth and other developments leading to a market over-tonnage/oversupply for both the regional firms and the outsiders and newcomers; and (b) the speed and aggressiveness with which the globalization phenomena expanded into the MERCOSUR. Cooperation or collaboration was neither natural nor common before the above two issues emerged within the region. On the contrary, rivalry, secrecy, pride, distrust, and a cautious optimism on what the regional future might bring did reign in the minds and spirits of both the corporations and their managers.

Cooperation and collaboration appeared as distant issues to be achieved amongst those who were considered as fierce rivals and even liable for the on-going market over-supply and its consequent fall in ocean freight rates. Knowledge sharing and transfer was therefore a totally internal issue. Sharing economic and market information was a matter to be dealt with only by a few within a limited corporate circle of trust. The main objective of the Corridor roundtables was to widen that circle to allow knowledge, cooperation, collaboration and leadership to emerge and evolve.

Edgardo Illia - Zim's director of operations based in Buenos Aires - commented ${ }^{74}$ : "When the over-tonnage became apparent and together with it the fall of freight rates and cargo volumes, we all realized that we were going to face a downsizing process, rather sooner than later. When we started to attend the roundtables I must admit I was totally lost as I used to regard all the attendees as rivals or in the best of cases as colleagues - provided they did not operate or traffic where my company operated. I was close-minded and went through many feelings that had nothing to do with trust build or knowledge sharing. I never felt comfortable with disclosing the real situation my company was going through before others. However, I found a more open-

\footnotetext{
${ }^{74}$ Note that all quoted comments in this thesis are translations from Spanish or Portuguese and have been checked for validity of meaning with each quoted person.
} 
minded behavior coming from my foreign-based principals who obviously did no have any resentment or bad feelings against other players - possibly because of their past global jointventure experiences with Hanjin and others companies. I learnt to share information and conceptualize others' information from my foreign bosses who also used to attend these meetings aiming at identifying potential partners. In other words, I did make up my mind from total indifference and mistrust to a gradual and growing acceptance towards concepts that sounded weird to my ears such as CoP, roundtable forums, knowledge sharing and transfer."

I remember having operated a dozen full-container ships when representing Zim Lines in the River Plate. We all felt proud of our fleet which by then was regarded as being technologically more advanced than that of most of our competitors. We all felt confident about the company's capability to further expand toward SAMWC and from there to the Asia-Pacific market - thanks to the driving force arising out of the ever-expanding MERCOSUR cargo flows.

All of a sudden we started witnessing the arrival of global companies searching for executives with regional business experience while announcing the launching of new services of global reach and state of-the-art technology. The market transformed so quickly that we saw our executives flocking to these new global recruiters while at the same time our cargo flows started spiraling downward.

To summarize, partnering seemed to be our only option to keep active in business as globalization showed no mercy to those lacking significant economies of scale or a given level of integration. Edgardo Illia commented further that the MERCOSUR Atlantic Corridor was for them like being in limbo as skepticism, rivalry, and possibly hidden agendas were suspected to be present; "I found myself very uncomfortable to share a roundtable with people I could barely stand out”.

He further carries on to admit or recognize that once the personal antipathy phase was somehow overcome (or at least tolerated) knowledge sharing started to flow in terms of informal conversations during occasional encounters or coffee breaks right before or after the roundtable forums were held. He also recognized that with the passing of time and the business context 
becoming increasingly threatening, they all started to gradually open their minds and share more vital information in a much more sincere and open way.

Carlos Milione was by then Multicargo’s CEO for South America - an Italian-based freight forwarding company who became one of the main cargo providers for Zim as well as very well known by the other three members of the JV. He was an active participant of the roundtable forums representing his leadership role of this freight forwarding company and their multiple logistic operations across Brazil and Argentina.

When asked about the way Multicargo formulated and implemented their business planning in a volatile and changing context, he replied: "Since we started operating in South America we never applied any linear model as we used to do in Europe but had to limit ourselves to drawing analogies and try to recognize patterns to better face the unexpected. In this regard the roundtable forums turned out to be an exceptional place to listen and conceptualize others' experiences and methods to face the unexpected - further to make strategic contacts in order to prepare the ground for potential alliances”. Mr. Milione's comments seem having a link with Courtney, Kirkland and Viguerie's (1997) true ambiguity concept where there is no solid basis to forecast the future, other than innovating and looking for new patterns. "As a logistic company, we were accustomed to do market intelligence; this is attending to every possible meeting; exchanging information with key market referents; capturing evolving situations and identifying new trends; raising daring comments to provoke others to make their statements and get them contrasted with others' opinions. So I found myself very comfortable attending the forums. However, it was hard for me to understand what the real value was for all those people attending the forums without showing any willingness to share information".

Edgardo Illia was also of the opinion that it was extremely unlikely to start sharing information or data in a sort of public event especially when apparently nobody knew well what they were doing there. "Everybody was very much expectant to see what the others were about to say or what kind of data and information was going to be disclosed. I found those events really closed and useless owing to its rigid formality that focused too much on corporate presentations without really addressing the upcoming trends or current problems. However, I must admit that most of 
my personal progress in terms of contacts and information flow was achieved through informal communications on a face-to-face basis, far from the stage or public exposure. Informal communication channels worked beautifully and actually proved essential in easing the JV that was later implemented”.

Alejandro Faliotti was Maruba's operational link with the other members of the JV as far as joint port operations and inland logistics were concerned all over the MERCOSUR ports. His role was crucial in organizing Sao Paulo's coordination center where three shipping companies and a huge road transportation firm had to bring all their operational and systems data to be coordinated and therefore optimized. Mr. Faliotti's view can be summarized as follows: "What I went through at the Corridor roundtable forums turned out to be much easier than having to deal and negotiate with the JV partners once the alliance was made. The globalization trend spread out all over the region so fast and so aggressively that one had to be blind not to realizing what was coming along with the impact on all and every of us here. The coming of the global carriers along with a tremendous excess of supply and a consequent fall in the ocean freight rates made it clear there was a need for a deep and fast reengineering process of their respective business for all the regional players. In our case it turned out to be simple: Maruba neither had enough economies of scale to compete with the cost efficiencies held by the global carriers, nor the needed horizontal integration to offset the global companies' on-shore logistic activities”.

Mr Faliotti argues that it quickly became a kind of learning organization perhaps because of the fact his company was regional and not global—and therefore was aligned with those who felt threatened by globalization. Garvin (1998, pp48-78) contends who contends that an organization cannot improve without first learning something new and that solving a problem, launching a service, or reengineering a process all require adopting a learning attitude on the part of both companies and individuals.

Mr. Faliotti further commented on the way he perceived the globalization impact on the regional business: "we all of a sudden became open and receptive not only to exchange vital information with the larger firms but also to make available our regional knowledge to the newcomers. I would rather regard this situation as a self defense attitude rather than a genuine desire on the 
part of the regional firms to pursue any managerial excellence criteria or diversification strategy. We also foresaw a unique opportunity not only to learn and capture the knowledge on operations from the global ones, but also to expand our services by making use of their existing global infrastructure at very competitive costs - which for them turned out negligible in terms of volume and for us was simply huge”.

It becomes apparent that the opening learning process for the regional companies was slow and hard at the outset regardless of how skeptical or optimistic their executives might have felt with the on-going system. However, they all seemed to open up and let knowledge sharing and transfer occur at a much faster rate and to intensify cooperation and collaboration as time passed and they faced rapidly approaching globalization forces.

Di Gregorio’s regional manager Mr. Federico Faliotti was in charge of representing the company before the roundtables and for identifying and initiating conversations with those potential JV partners. When asked for his impressions on the way leadership emerged in those forums he replied: "Leadership at the outset was brought to the roundtables by the Corridor brokers who were all the time highlighting the importance for all the attendees to take the lead of their own segments by displaying what their problems, limitations or even opportunities were to cooperate in search of larger economies of scale, associations, partnerships etc. Firstly, there were global companies with existing services in the region to take the lead; secondly there were global firms with little or no presence in the region; and finally the regional firms were the ones playing the role of followers though pretty active in terms of joining committees, teams, and working groups specialized in different segments of activity".

It becomes apparent here that the concept of the CoP was born somehow naturally - though it was indisputably driven by the leadership on the part of the global companies who held more experience and showed themselves much more receptive. "Leadership here had no connection with origin or nationality but that with knowledge and possibly international or global experience. When the time came for the regional companies to expose their weaknesses and strengths it was very clear that the leadership in conducting alliances and partnerships were on 
their side and not on the global firms - who appeared more interested in achieving a wider market coverage than finding managerial quality or assets differentiation”.

Sharp differences were identified when addressing the issue of cultural barriers and assessing whether they impeded or fostered the dialogue among the stakeholders. Mr. Edgardo Illia of Zim and Mr. Alejandro Faliotti of Maruba - both Argentine nationals - were of the opinion that the globalization threat flattened all possible differences in favor of a rapid and straight forward approach to the main topics. The other two JV executives held a different perspective. Di Gregorio’s Federico Faliotti - Brazilian national - categorized their Argentine colleagues as less open-minded and cautious than both his team and Brazilian executives in general. He regarded the Brazilian executives as more willing to share experiences and more open to disclose what it might be considered strategic business data.

Multicargo’s Carlos Milione - Italian national - was of the same opinion than Di Gregorio’s executive though highlighted the fact that despite their apparent initial mistrust on the functioning of the roundtables and the disclosing of key data and information, the Argentine executives were surpassed by the business context that demanded to find urgent alternative ways of action and therefore compelled them to leave any resentment or mistrust aside to give themselves a chance to reengineer by complementing other firm's services, partnering or finding a strategic ally to get integrated with.

One may infer at this point that for the Brazilian firms holding larger economies of scale and a more advanced technology may have granted them a (perhaps limited) sense of confidence to face the globalization wave in better shape with respect to knowledge, cooperation, and leadership.

The next section will draw some preliminary conclusions on this first case study.

\subsection{5. - Conclusions on case study \# 1}

I have participated in various roundtable forums almost from the outset of the Corridor and had the opportunity to witness the difficulty the attendants had to communicate and interact with their 
peers, competitors and participants in general. However, this situation was not too much different from that of any typical professional conference, symposium, or seminar regardless of topic. The following summarizes the case findings:

1. Forum attendees did not come to the forums to learn or selectively pick a piece of information on a specific issue. They attended with the intention to discover a new dimension for their businesses as well as to better understand the context they were operating in. However, and as earlier referred to by a global company representative, leadership was exerted by those who went through similar critical situations in other geographical areas outside the Corridor who had previously engaged in negotiating JVs and alliances of various kinds in business scenarios of perhaps greater cultural complexity - e.g. Asia-Pacific; Middle East.

2. Leadership seems first to have been taken by those global operators with both regional and global experience to be later be followed by global companies that held a lesser-relative degree of commitment to the region. However, an upcoming threatening business environment dominated by a growing excess of tonnage and a depressing ocean freight market seemed to ease all sorts of interactions and communications amongst at least a majority of the attendees. The launching of new global and fully integrated logistic services across the region can be considered as an astonishing experience for all those regional companies and executives accustomed to an almost linear-projected and legallyconstrained business context. This kind of executive was precisely the one that showed a relatively lower level of willingness to share knowledge when compared to those holding international experience or having lived comparable experiences abroad.

3. It was apparent that the openness of people to reveal data and information they believe was important, did not depend so much on their culture or nationality, but that was more related to their company's relative strength and competitive position in a given activity. Relative forces and market positioning appears have to played a role in influencing the executives' minds and attitudes at that time to expose, comment, or communicate their motivations.

4. All interviewees appear to agree with my observations that rivalries and personal mistrust tend to soften over time to eventually become resolved. This process led first to an informal and tacit face-to-face stage of preliminary communication, to later bring about a 
more formal and sometimes explicit knowledge sharing and transfer process in the form of a potential corporate cooperation, integration, alliance, or partnership of any sort.

5. The way CoPs became established and evolved within the forums and their members managed to actually overcome barriers such as: mistrust, envy and antipathy (relationship inhibiting); information ambiguity and knowledge viscosity (knowledge hording); cultural rivalry as well as many other possible relevant leader-follower aspects (commitment barriers). There was something about the way that these CoPs operated that helped build trust, build commitment, develop a general understanding of the threat facing these companies, and to focus a means to counter the threat.

6. There seemed to having been a mother or master vision concept present that eventually, one way or the other, pushed most attendees toward reengineering their businesses or to simply face commercial extinction. The impact of globalization can therefore be regarded as the real driver and trigger for companies of different size, structure, and segment to want to get together in search of a new business dimension. This process also brought about a tremendous business possibility for those secondary stakeholders that had no direct interest in any logistic segment but rendered consulting, engineering, legal, insurance, environmental, and further services. For them, their businesses being already more international in nature, the process the logistic community was going through meant nothing short of a business opportunity in itself to facilitate the upcoming organizational transformations.

7. It can be affirmed that even though the globalization wave was the main driver toward knowledge sharing and transfer that later led to a number of associations, it was also the critical high value traditional knowledge held by a number of regional operators combined with the existing cultural asymmetries between the outsiders and the regional players that helped the latter retain sufficient competitive value to therefore remain active in their respective fields - though with a transformed managerial and operational structure.

8. Leadership seems to have been initially exercised by those holding an extensive level of overseas experience as well as those companies that owing to their size and relative market position, felt secure enough to open up and expose their situations, views, and expectations through knowledge sharing. This sharing process did not naturally turn into a transferring stage until a certain level of trust had been achieved among the attendees. 
9. Globalization as such, seems to have sped up the process for the leaders and immediate followers to engage in a number of transformations that helped them overcome the upcoming challenges. Globalization also induced the laggards, often holding lesser relative market share and a weaker market positioning, to imitate the leaders and take positive action upon their businesses and structures.

Case study 1 illustrated how the Corridor leaders engaged in enterprise and how it acted as a trigger for a transformation project or program of changes. The next case study involves one of the leading participants of the roundtable forums (a global shipping firm with presence in the region) that could be considered an immediate follower though holding a strong market position and large structure (a railway company) - as well as a number of laggards represented by trucking companies of small operative range.

\section{3- CASE STUDY \# 2: INTRODUCTION}

This provides another example of the 'project' being a sophisticated program of work involving a business transformation entailing managing the change initiation as well as one that involved managing networks of players as well as multiple business re-engineering process improvements as outlined in Section 6.1, this involves a multimodal transportation transformation. Table 6.4 provides summary information about the case study.

Table 6.4- Sources of Data for Case Study \# 2

\begin{tabular}{|l|l|l|l|}
\hline \multicolumn{1}{|c|}{ Company } & \multicolumn{1}{|c|}{ Source } & Content & \multicolumn{1}{c|}{ Notes } \\
\hline Zim Line & $\begin{array}{l}\text { Operations Director for 10 } \\
\text { years at Zim; Operations } \\
\text { Manager for 6 years at } \\
\text { Delta Line; Multimodal } \\
\text { Manager for 10 years at } \\
\text { Elma. }\end{array}$ & $\begin{array}{l}\text { Knowledge sharing } \\
\text { and transfer strategy } \\
\text { within the roundtable } \\
\text { forums. }\end{array}$ & $\begin{array}{l}\text { Numerous comments and } \\
\text { exchange of opinions were } \\
\text { registered in view of my capacity } \\
\text { of Corridor promoter and Zim } \\
\text { executive. Notes and comments } \\
\text { were taken and periodically } \\
\text { reviewed and discussed with the } \\
\text { source from 1997 up to 2000. }\end{array}$ \\
\hline BAP & $\begin{array}{l}\text { Marketing Manager for 5 } \\
\text { years after holding various } \\
\text { positions at BAP on traffic, } \\
\text { operations, and quality } \\
\text { control for more than 10 } \\
\text { years. }\end{array}$ & $\begin{array}{l}\text { Reengineering } \\
\text { strategy: how to shift } \\
\text { from follower to } \\
\text { leader while } \\
\text { overcoming apparent } \\
\text { cultural inhibitors. }\end{array}$ & $\begin{array}{l}\text { A limited number of informal } \\
\text { chats held during coffee breaks in } \\
\text { the roundtable forums during } \\
\text { 02/1996 and 06/1996 - while the } \\
\text { land-bridge was designed and put } \\
\text { into practice. Also in my capacity } \\
\text { of Zim executive had access to } \\
\text { some deeper insights on BAP's } \\
\text { expectations and comments }\end{array}$ \\
\hline
\end{tabular}




\begin{tabular}{|l|l|l|l|}
\hline Refinerias De Maiz & $\begin{array}{l}\text { Commercial Director for 5 } \\
\text { years at Refinerias; CEO of } \\
\text { Kraft Foods; and } \\
\text { International Business } \\
\text { Director for Molinos Rio } \\
\text { De La Plata (all of them } \\
\text { large corporations with a } \\
\text { diversified global business } \\
\text { base) }\end{array}$ & $\begin{array}{l}\text { Tangible benefits from } \\
\text { CoP through } \\
\text { knowledge sharing. }\end{array}$ & $\begin{array}{l}\text { during operational coordination } \\
\text { meetings. }\end{array}$ \\
& $\begin{array}{l}\text { Occasional short gatherings during } \\
\text { roundtable forums, where practical } \\
\text { concepts on operational issues and } \\
\text { alternative opinions were } \\
\text { registered while ideas were } \\
\text { exchanged and assessed. Data } \\
\text { gathering took place between } \\
\text { approximately 6 months intervals } \\
\text { from 1997 and 1999. Data was } \\
\text { validated with the source by early } \\
\text { 2008. }\end{array}$ \\
\hline P\&O Ports & $\begin{array}{l}\text { Operations Manager for } \\
\text { more than 6 years and an } \\
\text { extensive experience in the } \\
\text { River Plate port business } \\
\text { for more than 2 decades. }\end{array}$ & $\begin{array}{l}\text { Two short informal conversations } \\
\text { held in 06/1998 as well as my } \\
\text { private observations of the } \\
\text { different roles P\&O adopted } \\
\text { according to who the participants } \\
\text { were. Data was checked with the } \\
\text { source in 2007. }\end{array}$ \\
\hline Zim Lines - BAP & $\begin{array}{l}\text { Corporate strategy. } \\
\text { Senior Managers }\end{array}$ & $\begin{array}{l}\text { Planning and coordination } \\
\text { meetings leading to the exchange } \\
\text { of concepts and opinions inherent } \\
\text { to the Corridor's contribution on } \\
\text { knowledge sharing and transfer. }\end{array}$ \\
\hline
\end{tabular}

This case study involves two direct participants and a number of indirect stakeholders or service providers without whom this project would have been impossible even to formulate. This project was about setting up a combined multimodal transportation system linking two continents and a number of markets spread out across Western and Eastern Europe with Chile in South America.

The SAWC market was (prior to the Case Study \#2 JV formation) normally serviced from the Mediterranean trade by various shipping liners deploying both full-container vessels and multipurpose ships ${ }^{75}$ that called regularly at a number of ports along western Europe (Spain / France / Italy) and a number of ports in Chile - mainly San Antonio or Valparaiso. San Antonio was by definition a container terminal designed to efficiently handle the loading, discharging and transfer of containers from and to full-container vessels.

Valparaiso fulfilled both roles - container and break-bulk cargo terminal. Actually Valparaiso turned out to be more efficient in handling refrigerated and dry break-bulk cargo while the

\footnotetext{
${ }^{75}$ Multi-purpose ships are those designed to carry a variety of cargo types such as containers, loosen pieces of cargo, refrigerated cargo, and in some cases liquid bulk cargo. They were design to complement the full-container vessels servicing only the container segment.
} 
container segment kept predominantly dominated by San Antonio. However, Valparaiso became congested during the export season of reefer cargoes often leading the container segment to entirely being operated from the San Antonio container terminal.

Both San Antonio and Valparaiso lie equidistant to Chile's capital and most important industrial pole - Santiago. Import and export goods, to and from the Mediterranean market, were met through the two mentioned ports by sea-going full-container vessels. Typically these services used to call at the ports of Genoa, Leghorn, Naples, Marseille, Valencia, and Barcelona in Europe further to connecting with Eastern Europe markets by utilizing any of the mentioned ports as hubs or transshipment ports towards SAWC. Even though the SAWC market embodied Chile, Peru, Ecuador, and Colombia, there were few existing liner shipping services because of the relatively lower available cargo volumes when compared to the SAEC market where. Brazil was the principal market driver of the SAEC that attracted many shipping companies even further south into the River Plate by adding greater economies of scale.

Brazil's GDP was by then 5 times Argentina's, while the latter's GDP was 3 times that of Chile. This lesser relative GDP scales on the SAWC along with the absence of a huge or leading regional GDP explains why the SAWC trade was less serviced than the SAEC. One may infer that this was the reason why the globalization wave focused first on SAEC to only later reach certain specific segments on SAWC.

This market situation (along with the Panama Canal costs ship-owners had to face to have access to the Pacific Ocean) led to a level of ocean freight rates of greater relative cost as compared to the existing levels on SAEC's Atlantic Ocean. While the trade between the Mediterranean and SAEC market offered a sailing every 2.5 days, the Chilean ports were called at every 8 days. This situation led to less competition and consequently to a higher relative level of ocean freight rates, longer transit times, congestion surcharges, as well as recurrent waiting times at the transshipment ports in Europe.

The alternative to competing with this situation was to set up a door-to-door service linking both west and east European ports and inland locations with Buenos Aires port in the River Plate to 
further continue on a land-bridge mode up to the industrial pole of Santiago. Detrimental factors for the SAWC market included the relative lack of a competitive sailing frequency along with a higher relative cost and longer transit times through the Panama Canal to reach Chilean ports. However, this presented an attractive window of opportunity to exploit finding a land-bridge alternative.

The Mendoza market in Argentina was of critical importance in the development of the economics of a land-bridge as it provided strong and ever-growing cargo flows that could help in utilizing the containers that had returned empty to Mendoza from Chile having exported regional goods. While most imports to Chile demanded 40 foot containers - used for lighter cargoes exports from Mendoza mostly required 20 foot containers - designed to carry heavier cargoes. Therefore a growing imbalance of empty containers threatened the whole logistic rational of the land-bridge which was eventually worked out by adapting the relative heavier cargo flows ex Mendoza to partly fit into the 40 foot containers.

This sub-utilization of the equipment was aimed at achieving an acceptable equipment turnaround-time that enabled the shipping companies to profit from the horizontal integration without running the risk many ship-owning firms incurred in the past when dealing with long TAT ratios and large numbers of lost equipment on worldwide basis. This efficient utilization of the equipment counted on the cooperation of the interested parties who accepted reengineering their operations and lowering their margins to remain competitive enough to capture a new promising market. Thus Zim Lines, BAP, and a number of contracting parties showed a high degree of corporate cooperation and leadership to make the land-bridge competitive. The next section will explain in better detail the way this land-bridge worked and the rationale behind it.

\subsection{1. -Structure of the alliance}

Figure 6.2 illustrates the nature of this case study alliance. 


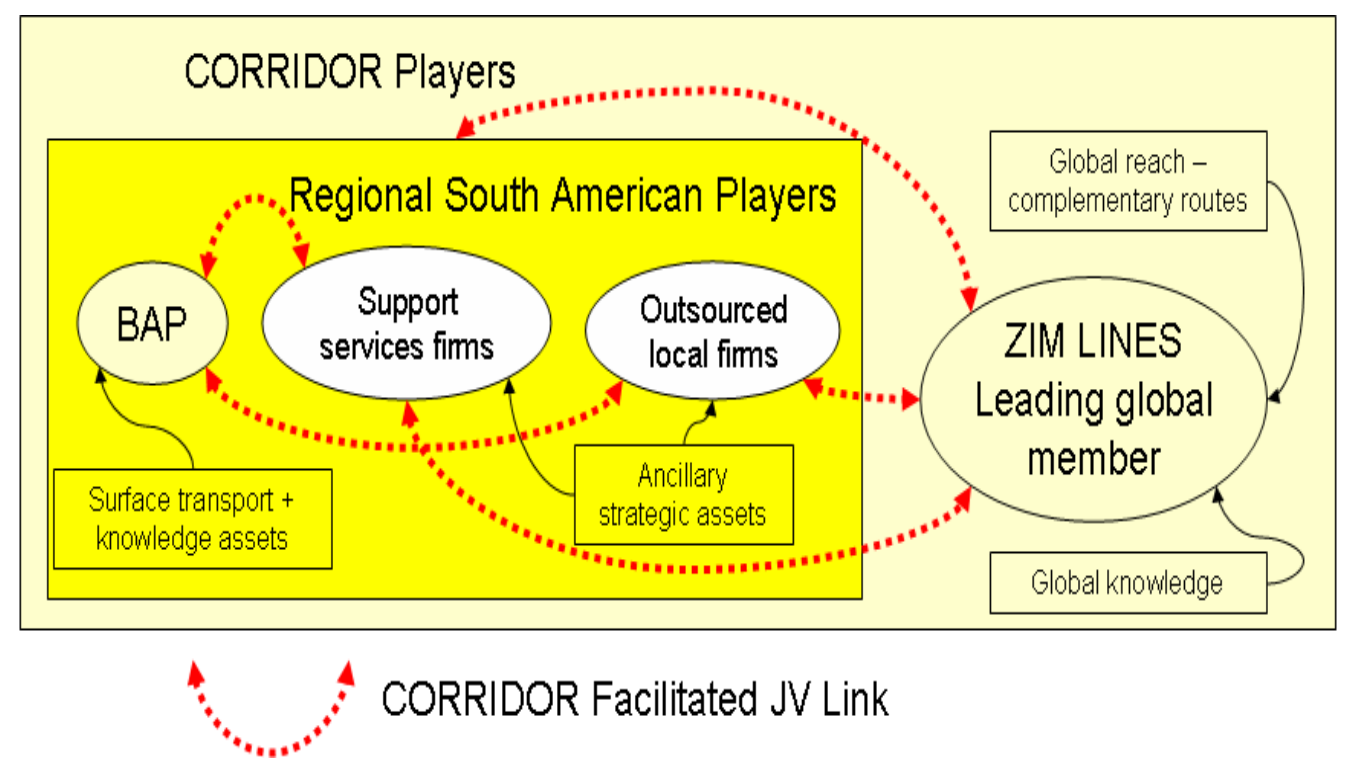

Figure 6.2 : Case Study 2 Alliance Relationships

Two firms were involved from the beginning of the project while a number of companies that fulfilled different roles gradually joined to set up a strategic alliance for the duration of the project. One of the leading companies was Zim Lines whose structure and scope of services was duly exposed in the first case study. Zim Lines was in charge of providing ocean transportation services from the entire Mediterranean market up to its regular port of call in Buenos Aires, Argentina. While western Mediterranean ports were serviced directly, both eastern Mediterranean and northern African ports were serviced either via Barcelona or Marseille. The port of Haifa in Israel was not only used as a second transshipment port but also as the entry gate to Chile for the entire inland Eastern Europe market. Therefore the land-bridge represented a huge market to focus upon and to develop by taking advantage from the by then on-going inefficiencies on SAWC market.

The other leading company was by then named BAP railways or Buenos Aires al Pacifico rail services. The Buenos Aires and Pacific Railway (BAP) was one of the Big Four broad gauge British-owned companies that built and operated railway networks in Argentina. This company provided railway services from their Buenos Aires railhead up to their terminal in Mendoza city about 1000 kilometers west of Buenos Aires and lying on the Andes Cordillera. 
Linking road transportation services between the port terminal and Buenos Aires railhead was an operation almost completely outsourced to a number of independent domestic operators, further to BAP that also provided a number of trucks of their own to assure a minimum safety level of cargo flow operations. Trucking services from Mendoza's railhead to Chile and back were entirely outsourced to a number of bi-national trucking companies from both Argentina and Chile.

In addition to the road transportation component of the alliance, a number of ancillary services were either outsourced or integrated in the alliance including ship-agency services, warehousing operations, crane operations, customs brokerage, insurance services, cargo surveys, and others.

Possibly the strength of the alliance laid in the complementing role of the various players to render a competitive service on a sea-road-rail mode aiming at outperforming the traditional direct ocean shipping services linking the Mediterranean market and the Chilean ports. The landbridge achieved a lower level of freight rates and therefore a more competitive service profile. An important feature was the existing transit times were shortened by $1 / 3$ thanks to holding a better frequency of sailing ex Europe as well as shorter distances and no Panama Canal to get through.

Europe is to Argentina what the Asia Pacific region is to Chile in terms of distances and access to logistic services and operational combinations. The economies of scales between Chile and Argentina will always make the latter being better serviced than the former.

An additional advantage was given by the fact that by the time the alliance was set up and the land-bridge established, shipping companies rendered their services on a port-to-port basis without involving themselves in additional services that could have granted them both more control on the cargo-routing decision making and possibly achieving higher margins. Therefore horizontal integration was practically non-existent as the market remained untouched for so long that every segment did develop by looking inwards and without watching global trends in similar markets. 
The way this group of shipping companies rendered their services in SAWC by the early 90s, was representative of an on-going business environment characterized by their firms, shipping agencies, ground transportation companies, warehousing firms, port terminal operators, customs brokers and many other ancillary services facing little competition within a cartelized business context, modest innovation, fleet ageing, protective cargo legislation, no clear market leadership of any group, and very little natural integration.

On the railways side, Argentina had South America's most extensive railway network that despite connecting with the gauges of all the neighboring countries (Chile / Bolivia / Paraguay / Brazil) concentrated only on carrying domestic cargoes with no projection outside their domestic market. Analogies can be found in both Chilean and Brazilian networks, for reasons of geopolitical nature and beyond the scope of this thesis.

Therefore it becomes apparent that the land-bridge can well be regarded as the initiator of a new concept that brought about a complete new horizon for developing land-bridges within the MERCOSUR region. The following section will bring to the reader's attention some operational particulars of both the land-bridge and its players further to their opinions and views on the way the Corridor has played its role in paving the road of a good part of the alliances and JVs across the region.

\subsection{2. - The corridor as a facilitator}

Once the globalization process affected the region, practically all on-shore transportation operators as well as the ancillary services found themselves non-integrated, working isolated from one another, and functioning within a world where concepts such as strategic alliance, innovation, differentiation, and diversification were not seriously considered. This situation happened to be common on both sides of the Cordillera.

The very first example was Zim Lines that operated a number of lines with 12 vessels that soon had to partly flagged-off to reengineer their structure as it was explained in Case Study \#1. Secondly it was the railway company that focused all its efforts on the domestic market as if a 
country's economy (in this case Argentina) was immune to any kind of foreign-based economic competitive trend. They believed in a sort of linear market model with no outer economic interference to successfully expand by strengthening their services either regionally or even internationally. This was exemplified by their reliance on all necessary legacy infrastructure elements that the British had envisioned and designed a long time ago in the country—plenty of rolling stock; direct access to leading industries; direct access to port terminals; access to strategically located markets and industrial poles; fully operational rail-heads; cranes; trucks; etc.

A similar situation can be illustrated by a number of road transportation services which, before the land-bridge was launched, did not even try to complement their services with that of the various railway companies to better cope with the available market segments. Each trucking company focused on their base Province and dedicated themselves to render services to and from their respective Provinces.

This situation hindered the operators from realizing how low entry barriers were to their markets or their weaknesses relative to larger scaled competitors. Among the ancillary services the shipagencies were the ones who showed a total passivity towards innovative service trends. This was despite the fact that they generally represented a business sector which had the closest links with the shipping business. One would have expected this kind of access to knowledge of emerging global trends to have penetrated the consciousness of these ship-agencies.

Edgardo Illia - Director of Operations at Zim Lines in Buenos Aires - highlighted the fact that for them meeting logistic operators of different kinds (other than ship-owners) was a truly enriching experience; "Being myself fully committed with the shipping business itself and with the company I represented, sharing views and opinions on the upcoming trends with different sectors' colleagues, as well as witnessing the way the attendees tended to relate to one another regardless of the sector they were engaged in, was truly demonstrative of the Corridor potential. The inhibition I experienced when facing competitors on the shipping business vanished almost completely when talking to railways, trucking companies, feeding or river barge firms”. 
Walter Markevich was BAP’s Marketing Manager and was perhaps a dynamic activist in pursuing some sort of complementation that might enable his company increase the cargo volumes and intend reaching some innovation degree. "I was personally convinced that with the coming of the large global carriers to the region and the relative loss of importance the domestic market held to our company, we were compelled to look for some sort of reengineering. By the time we joined the Corridor and started attending the roundtable forums we did not have a clear idea of what we were looking for or what to expect from it. We just intended to experiment with offering some approaches to a number of shipping companies to see whether they might be interested to outsource their inland door to door services through our railway network. Nothing very innovative I presume. However, one of these shipping firms brought up the idea of a landbridge and all continued from there as we all know. I would say we pretty much followed the attitude and behavior others were taking by approaching colleagues and competitors to exchange views and opinions”.

When asked about the best way to contact potential allies and if tacit or explicit information was as a result, best to their ends. Both Edgardo Illia and Walter Markevich replied that exchanging tacit information delivered in an informal fashion was definitely best used to sound out potential firms. Face to face contact was primarily of paramount importance to establish the relationship that enabled them to later look into detailed facts and figures. Cultural barriers to knowledge transfer were not specifically identified but were categorized simply as cultural differences that had an impact on timing and decisiveness of engagement, but appeared to have no tangible effects on conceptual or central issues.

In this regard Walter Markevich emphasized that when approaching regional companies to exchange opinions and views on a specific market situation, he only later realized that they actually were sounding each other out without being really aware of it. In this exercise he noticed a certain resistance on both their colleagues and themselves to freely expose their arguments though he attributed this phenomenon to be a rather natural human behavior and not too much of cultural inhibitors or barriers of any kind. 
Refinerias De Maiz is an Argentinean top ten exporter of agricultural products. Marcelo Rosatto, Commercial Director, was the company's representative at the roundtables and an active participant in all those topics concerning regional logistics operators and available physical infrastructure. He stated that "In my capacity of exporter I never attended the roundtable forums to find a business partner with whom I could formalize a strategic alliance. On the contrary, I felt free to expose what our operational limitations were and claim from the participants to help us overcome those problems. Therefore I've never gone through any sense of cautiousness or mistrust on the part of the attendees when sharing information. I must admit that the complexity of the problems that were often exposed resulted for me a bit difficult to comprehend as they were entirely logistic issues beyond my scope of interest. I was in fact trying to make sure these companies found the solutions to our global problems and not the other way around".

When asked about how he would regard the roundtable forums should his company have to look for a partner he replied, "It was not my case but if I had to propose an event or (business knowledge exchange) system for my company to attend and try to identify a suitable firm to get it vertically integrated in with, no doubt it would be the Corridor roundtables. However, I imagine their apparent positive attitude toward sharing data and keeping open and frank in their views and perspectives may have been influenced by the on-going business context. My company is a leading exporting firm and therefore I do not imagine the Corridor roundtables being of any potential other than a suitable forum to expose my company's operational bottlenecks and naturally demand solutions from them. I do not see an agricultural exporter of our size committing to a vertical integration process with a company we came to know in a forum of this kind because of two things: a) we do not need it as the degree of integration in a business like ours is in fact pretty low, and b) we have enough global contacts to undertake an integration strategy should we decide to".

When asked about the extent to which he came to comprehend what was discussed or exposed in the forums, he explained that he was aware that he was witnessing a rather rich or complex knowledge transfer process amongst the logistic companies though he stated he was not really qualified to follow the discussions. Even though it becomes apparent that, knowledge stickiness was a factor for Refinerias De Maiz, the Corridor roundtable meetings became useful for it to 
investigate various business problem solutions that otherwise would have incurred a waste of resources (in terms of time and personnel).

A clear leadership role seemed evident for many of the participants in the sense that companies who exerted control on the cargo routing decision-making, were the ones showing leadership in the discussions, while the all other firms rendering supporting services regardless of their size and structure seemed to be the followers.

This was apparently the case of the railway company BAP which, despite counting on a huge federal structure within Argentina as well as regional connections, had little influence on the cargo routing. BAP's marketing manager defined the role his company adopted during the roundtable forums: "It turned out to be a bit embarrassing I must admit but we had no option. In terms of leaders and followers we definitely were followers despite having lots of physical and human resources at our disposal. The reality showed us we had no control on the cargo routing though we could become an essential part of the chain should we follow the shipping companies and ally to one or two of them to better operate our rolling stock".

For the railway company it becomes apparent that the roundtable forums gave them the opportunity to not only get in touch with potential allies but to also better face the impact of globalization, and to also realize (and most importantly to conceptualize) their real role in the market.

P\&O’s operations manager Carlos Barbero had a partly coincident opinion as regards who led and who followed in the discussions: "We sometimes adopted a clear leadership position while in other occasions we found ourselves half way in topics we had nothing to say but, owing to our structure and economic importance for the region, we could not be seen as followers".

He commented on the various topics they had a say in by making a clear distinction between those they had control over and those they had little or no control over: "Naturally Zim was our client in Buenos Aires and we were compelled to listen and follow their directives. However, even though the railway company had direct access to the berths to operate alongside ships and make 
the whole land-bridge operations really efficient, it was up to us to permit this operation by negotiating with the port railway union. So sometimes we just felt ourselves to be followers with a say on our client's planning, while other times we were clearly the leaders".

P\&O's terminal operations manager opinion on the Corridor as a facilitator seems to be not that positive or at least a bit ambiguous: "I personally do not believe in companies exchanging information and key data. I believe the roundtables were a good place to interact and develop personal relationships at a corporate level, but by no means proved useful to develop a solid business strategy or a place where someone could pick up a challenging idea”.

However, the land-bridge seemed gathering all the variables of a challenging concept while the port terminal also seemed having benefited from additional traffic which otherwise would never have likely been realized. When asked about the leader-follower relationship and the real value of the Corridor itself, Zim's operations director stated: “I do not really know whether we were leaders or followers. I can only say we were all immersed in a kind of anxiety that led us to find ideas, concepts and elements that could help us identify a way of achieving larger economies of scale while remaining competitive. We were aware of what was coming and that we had to somehow reengineer to get by. I must admit that we could have approached the railway company long before meeting them at the roundtable. However I doubt very much that an approach would have evolved the way it eventually did as the Corridor gave us the unique opportunity to exchange lots of tacit information that later turned into explicit. Face to face contact was also of paramount importance to face hidden agendas, preconceptions of any kind, and to build trust. At the end of the day we all were members of the same Club".

It becomes apparent that there is no single current of opinion as the way each executive felt the pressure of the globalization impact. It also has to do also with the size and structure of their organizations as well as the role their own organizations were taking while in the roundtable forums. The next section will address some preliminary conclusions. 


\subsection{3. - Conclusions on case study \# 2}

A number of elements can be identified from the comments of the interviewees as regards what they perceived and the way they felt while attending the roundtables:

1. Lack of inhibition: inhibition to expose, interact, and eventually make data and information available tended to significantly soften and even disappear when interacting firms were not directly competing but rather offering complementary services. A shipping company, a shipping agency, a railway company, and a number of small-size local range road transportation operators did not offer much resistance to interact and generated a positive outcome. At this point one might infer that the information complexity or its attached potential knowledge viscosity did not impede players from reaching a win-win situation despite the unique nature of their respective businesses. In other words, the above might well suggest that cooperation and collaboration amongst the interested parties must have played a role to make inhibition negligible.

2. Lack of focus: despite the railway company being a rather large and well established organization, its management did join the Corridor without being really aware of their reasons to do so or the goals to be attained. This suggests a possible link with their stateowned management style and that consequential inertia was involved - all were immersed in a kind of lack of strategic thinking and therefore motivation. I had the opportunity to witness this aspect to happen rather often with state-owned or provincial-owned firms who seemed more interested in writing reports on their political views on the forums than to interact and exchange information and opinions. This aspect no doubt needed special attention from the Corridor brokers who were in charge of fostering direct contact and dialogue as to let knowledge emerge and flow amongst the members.

3. Cultural cautiousness: this is an aspect that even though it cannot be regarded as a knowledge transfer inhibitor in view of an existing rather homogeneous regional cultural profile, it might have slowed down the interaction of both competitors and firms from complementing logistic fields. The information registered from the interviews suggests that there was an intermediate stage where the participants took their time to perceive certain signals to get them processed and reflect upon these and to only later let an avenue of acceptance and further dialogue continue. 
4. Enhancer roles: the participation of a multinational agro-exporting company whose main interests were based on improving their own export logistic channels rather than looking for partners or potential associates to vertically integrate with, became a driver to the forums. Although the forums were thought of as entities for companies and individuals to share and transfer their knowledge and leadership to emerge, in my opinion this led to a general betterment of the Corridor roundtable system. The presence of a powerful outsider seems to have exerted additional pressure for the logistic players to innovate and come up with solutions while their interaction performance was being watched or somehow monitored by participants. Some of these logistic players found themselves compelled to present themselves as being active and resourceful before those who were possibly their main client or could become a potential customer in the near future. The above suggests that a passive attitude on their part while in the forums could have turned out counterproductive and that the presence of an outsider might be regarded as a powerful incentive or driving device to generate positive outcomes within the respective CoP.

5. Knowledge stickiness: difficulty in entirely understanding the topics that were regularly discussed on the roundtables seems to find very clear distinctions by participant segment. Outsiders tended to focus on their own interests without really interpreting the value of the exchanged information, while the complementing segments also found difficulty in absorbing their colleagues' knowledge despite the fact they operated under comparable logistic parameters. The Corridor brokers also had a task here of easing the information flow and forming interdisciplinary CoPs capable of letting interdisciplinary knowledge sharing and transfer occur for the benefit of all.

6. Leader-follower relationship: it seems to depend more on who held a greater relative power over the cargo-flow decision-making process and what their relative market positioning was at a given point, than on the company's structure, investment endowments, or managerial expertise. A sense of security on the part of those global companies seems to have dominated the leadership scene at the outset of the process. This suggests a direct link between the leadership group's influence stemming from their confidence of power and motivation to engage in knowledge sharing.

7. Direct interaction: face-to-face interaction seems to be the preferred and most efficient way to initiate a business relationship leading to a later exchange of information, views, 
and opinions - either tacit or explicit. Direct informal contact suggests it might also be a better way to deal with information stickiness.

8. Indifference: a certain degree of indifference becomes apparent in those global corporations that attended the forums in their quality of well-established and financially solid entities aiming more at following the trend of the systems rather than being part of it (e.g. P\&O PORTS).

This case study seems to bring to light a few new elements the previous case study did not expose so clearly. These are: enhancement impact of an outsider organization may have on the roundtables; the relatively low cultural barriers that might slow down the knowledge flow; and the indifference on the part of large corporations. These all seemed to be problems needing to be closely monitored and obviated in the Corridor to allow a more efficient and possibly faster interaction to happen — given the threatening business context. Leadership, knowledge, and interaction seem to share some common factors with the previous case.

\section{4 - CASE STUDY \# 3: INTRODUCTION}

The railway cargo transport segment, during the early 1990s, was a very much isolated or nonintegrated business in the various countries that made up not only the MERCOSUR Corridor Project but also the entire region. Brazil, Chile, Argentina, Bolivia, Uruguay, Paraguay were all countries where to a lesser or greater extent the railway played a significant and important role in their respective national economic development. Table 6.5 describes the following sources of information.

Table 6.5- Sources of data for Case Study \# 3

\begin{tabular}{|l|l|l|l|}
\hline Company & Source & Content & \multicolumn{1}{c|}{ Notes } \\
\hline $\begin{array}{l}\text { Mercosur Trade } \\
\text { Center }\end{array}$ & $\begin{array}{l}\text { President; Director } \\
\text { of Sao Paulo-based } \\
\text { Sebrae; Director of } \\
\text { Sao Paulo-based } \\
\text { Fiesp; Director of } \\
\text { Olam. }\end{array}$ & $\begin{array}{l}\text { Internationalization strategy } \\
\text { and consulting role. }\end{array}$ & $\begin{array}{l}\text { Occasional and formal meetings } \\
\text { were held from 02/1998 to } \\
12 / 2000 \text {. Notes were taken and } \\
\text { messages were exchanged on } \\
\text { concepts, processes, and } \\
\text { methodologies on CoPs, } \\
\text { knowledge sharing and } \\
\text { stakeholders integration. Data } \\
\text { was validated with the source by } \\
\text { early 2007. }\end{array}$ \\
\hline P\&O Ports & $\begin{array}{l}\text { Operations Manager } \\
\text { for more than 6 }\end{array}$ & $\begin{array}{l}\text { Observation role on ALL's } \\
\text { learning and knowledge }\end{array}$ & $\begin{array}{l}\text { Several short informal } \\
\text { conversations were held and }\end{array}$ \\
\hline
\end{tabular}




\begin{tabular}{|l|l|l|l|}
\hline & $\begin{array}{l}\text { years and an } \\
\text { extensive experience } \\
\text { in the River Plate } \\
\text { port business for } \\
\text { more than 2 } \\
\text { decades. }\end{array}$ & internalization process. & $\begin{array}{l}\text { tended to confirm my own } \\
\text { observations. Specific notes } \\
\text { were not needed but that my } \\
\text { others' notes were confirmed } \\
\text { and subsequently highlighted fir } \\
\text { future reference. Data was } \\
\text { validated with the source in } \\
\text { 2007. }\end{array}$ \\
\hline Multicargo & $\begin{array}{l}\text { CEO for South } \\
\text { America for more } \\
\text { than 10 years; } \\
\text { Country Manager } \\
\text { for more than 15 } \\
\text { years at K+N, in } \\
\text { charge of ocean, } \\
\text { ground, and air } \\
\text { transportation } \\
\text { departments. }\end{array}$ & $\begin{array}{l}\text { Observation role on ALL's } \\
\text { absorptive capacity. } \\
\text { held and impressions and } \\
\text { insights were corroborated on } \\
\text { regular basis at both the } \\
\text { roundtable forums and on } \\
\text { business meetings between 1996 } \\
\text { and 1997. Content was checked } \\
\text { with the source and validated } \\
\text { during 2007. }\end{array}$ \\
\hline $\begin{array}{l}\text { Regional } \\
\text { Procurement } \\
\text { Manager with more } \\
\text { than 15 years of } \\
\text { work at Skanska. }\end{array}$ & Corridor potential outcomes. & $\begin{array}{l}\text { A single informal lunch meeting } \\
\text { was held by late 1999 while } \\
\text { working for the Corridor to } \\
\text { ascertain Skanska's actual } \\
\text { involvement and views. Data } \\
\text { was validated with the source by } \\
\text { late 2006. }\end{array}$ \\
\hline
\end{tabular}

This case study embodies two independent railway companies that operated in a single country but that, at a given point in time, were jointly sold to a major railway conglomerate of a bigger regional player. These two railway networks were Argentina's BAP - previously described - and Ferrocarril Mesopotamico. While the former run westbound to Mendoza and Chile, the latter run northbound connecting Argentina’s northeast with Brazil’s southeastern region.

The new owner was Sao Paulo-based America Latina Logistica (ALL) which, while also already being a railway operator in Brazil, acquired these two strategic networks in Argentina with the aim of playing a regional role in railway logistics. Thus, ALL counted on the necessary network to duly interconnect the major industrial poles of the region by linking Sao Paulo and other coastal Brazilian industrial areas (Sao Bernardo; Curitiba; Rio Grande; Porto Alegre etc) with Buenos Aires and Mendoza in Argentina, as well as with Santiago’s industrial pole in Chile. Later, ALL acquired a new railing network connecting Sao Paulo with other industrial areas further north in Brazil until reaching certain locations which made the railway unbeatable in terms of cost per ton $/ \mathrm{km}$. 
The stages of ownership are illustrated in Figure 6.3 below.

\section{Stage 1: Argentine \\ Federal Government owned}

Stage 2: Argentine

owned but privatized

Stage 3: Merged and owned by Brazilian railway AMERICA LATINA LOGISTICA (ALL) Headquarted in Brazil
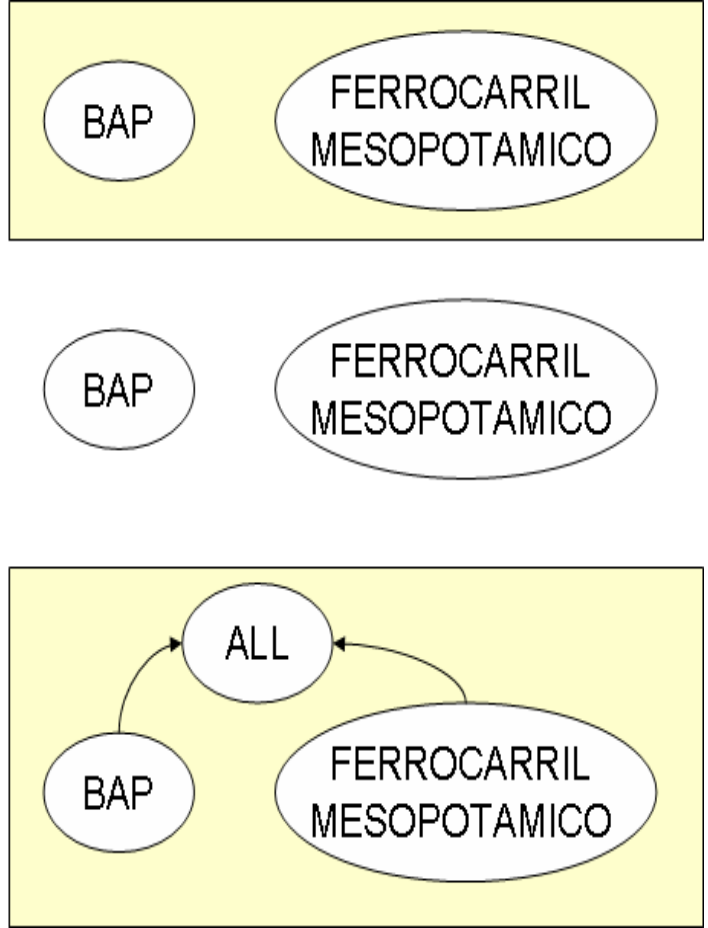

Figure 6.3 - Three stage ownership of case study railway organizations

Even though these two Argentine-owned railways were in private hands by the time they were sold to Brazil-headquartered ALL, they belonged to the federal government - perhaps for too long as they carried over all the operational problems one could possibly expect to deal with in a public company (e.g. lack of investment; obsolete rolling stock; overstaff; inadequate information systems; inefficient marketing and sales structure; highly unionized; highly politicized; etc).

Most of these topics were not even intended to improve as the sale to private Argentine owners was just a political move to later pursue a better sale to a foreign-based regional operator - ALL company. Thus, ALL inherited a long list of operational problems in Argentina which tended to aggravate ALL's own operations as it was not an international or even regional operator, but just a domestic operator within the vast Brazilian geography.

Consequently both ALL and their newly acquired networks in Argentina shared many common problems. While the Brazilian network showed itself to be more efficient in all those 
operationally-related aspects of the business, the Argentine networks not only had to be completely reengineered from an operational standpoint, but also had to open up their vision of the business together with their new Brazilian owners. Each company entering the ALL umbrella organization shared a totally domestic vision in their respective markets or countries.

Further to sharing many of the operational problems and despite being pretty active in their respective inland distribution segments, the two networks failed in accomplishing any serious degree of horizontal integration as far as trucking, warehousing, customs clearance; rail-head operations, or distribution services were involved. An additional condiment to this apparent difficult situation was given by the little or practically no control on the cargo flows decisionmaking process other than the domestic portion which they used to profit from based on the two countries’ long distances and a rather captive domestic market.

While the barging segment in the Parana-Paraguay waterways in Argentina was exclusively foreign-oriented, both the coastal barging and the Amazon River barging services in Brazil were to a great extent coping with the overseas markets. Little domestic coastal barging was carried out in Brazil despite the enormous distances that might make it highly advisable in view of its evident transportation economics.

The next section will address in more detail the challenges ALL had to face and the nature of their strategic plan I had the opportunity to contribute too.

\subsection{1 - Regional expansion and strategic plan}

The following lists the main challenges this company had to face when incorporating their two new westbound and northbound services in Argentina;

* Senior management holding a significant amount of tacit knowledge of a technical and operational nature, as well as a great difficulty in transfering this knowledge - mainly due to their immediate previous bad experience attributed to fear and mistrust on the part of the existing management with respect to the new private Argentine owners; 
* Non-existent marketing knowledge other than the one exclusively related to the domestic markets linking Buenos Aires industrial pole with Mendoza city in the west and Argentina's eastern cities reaching up to the border with Brazil.

* No agreement of any sort or operational complement with any of the Buenos Aires port terminals or with Buenos Aires up-river terminals which together offered more than a dozen opportunities to expand and grow beyond the domestic market.

* Overstaffing and a very strong presence of in-house union representatives as a result of having belonged to the government for too long.

* High level of inter-provincial politics with respect to the social role a railway had to fulfill with a great number of stakeholders in every single Province the railways went through.

Outdated locomotives, wagons, and poorly functioning or suitable rolling stock in general which made its operations highly inefficient.

* Non-operational rail-heads, inadequate transfer facilities and a high need of cranes and further transfer equipment.

Based on today's world market principles and capitalistic visions, one may easily infer at this point that there was a complete lack of vision, or at least a severe lack of talent and leadership, to allow these three strategic railway networks to even cope with the domestic markets. However, the domestic markets were by then generous for carriers that operated them. The region in general appeared to be closed or not receptive to foreign investors to enter their market and so this made the whole system fertile for inefficient operators to develop within a cartelized business context.

All this naturally tended to quickly change once the globalization wave of change approached. Both the legal framework and regional market favored opening up to foreign investment leading the whole business system towards needing more integrated and efficient companies. Thus, ALL had not only to renovate both their inherited assets and structure, but also to reengineer their vision and management with respect to the way they should be coping with the globalization process both in Brazil and Argentina. 
At this point, identifying and defining the way to influence the cargo routing decision-making processes became key drivers to further formulate a strategic plan that could enable the organization to get rid of its typical dependence on the shipping line that a railway company normally held. It was and still is the shipping line that primarily keeps contact with the exporter and importer and therefore exerts a high level of decision-making on the cargo-routing aspect. This only became apparent to ALL once clearly exposed in the roundtables. Once this phase had been completed, then recruiting the right people to formulate and implement a business plan was of paramount importance for the company in their intent to gain control of the cargo flows.

The Corridor roundtables proved to be of great use for an entirely domestic-oriented company with no international experience. It allowed the exchange of data and information along with countless face to face interactions ALL executives had with other segments' executives. Having experienced the way others were reengineering themselves to rationalize their services, optimize their assets, and restructure their management made them understand the main variables of the regional logistic market along with the international potential.

The regional railway company subsequently adopted a strategic plan that went beyond expectations, despite their management's modest exposure to international competition. As well as quickly advancing on all kinds of horizontal integration initiatives with trucking companies, warehousing facilities, and port terminals, they also engaged in setting up their own international structure instead of entirely relying on potential agreements with the shipping companies.

Such a structure meant initially to appoint a number of agents worldwide to sell door-to-door container services as a way to exert some control on the cargo routing by contracting the services of a number of shipping lines. At the outset this strategy turned the railway firm into a direct competitor for the shipping lines servicing Saec, as they were selling both export and import ocean freight services and inland transportation beyond the MERCOSUR region. 
However, over time, the railway became another significant $\mathrm{NVOCC}^{76}$ in the market enabling the shipping companies reduce their marketing staff in those locations where the railway's commercial structure had substantially grown. The second stage of their strategy was based in setting up their own regional commercial office in the main overseas markets to better monitor their growth and exert controlling functions on their appointed agents.

While their appointed agents were spread out all over the main global markets (European Union / $\mathrm{NAFTA}^{77}$ / Asia-Pacific region / Latin America), it kept a reduced number of regional headoffices aiming at monitoring the market trends and conducting negotiations with the port terminals, trucking companies, inland depots, and other suppliers as well as deepening the relationship with their appointed agents and shipping companies.

It becomes apparent that there was a significant shift in the way ALL operated and how it decided to cope with the new business scenario. From being first a domestic company to later focus on the region with neither regional nor international structure or experience and gain access to the global market cannot be considered as a natural managerial process.

On the contrary, it was the synergies found in the roundtable forums along with a knowledge transfer process that took place among the various sectors' representatives. Those interactions seem successful in that they proved useful to let them undertake a deep reengineering process of their business and a radical change of vision. The next section will address some executives' views and opinions on the influence the Corridor roundtables actually had in the entire process.

\subsection{2. - The corridor as a facilitator}

Roberto Cristaldi - Mercosur Trade Centre President (MTC) and consultant to ALL during the acquisition process, highlighted the learning attitude of all the railway firm's executives that used to attend the roundtable forums. "ALL executives possibly had one of the most willing attitudes toward incorporating knowledge and interacting with peers from other companies and segments.

\footnotetext{
${ }^{76}$ NVOCC: Non-vessel operating common carrier means the kind of company that handle other companies' cargoes but that doesn't own or operate a vessel as a typical ship-owner does.

${ }^{77}$ NAFTA (North American Free Trade Association): it means a regional free trade association that includes Mexico, USA, and Canada).
} 
They integrated with whatever committee they were invited to participate and join with no reserves in exposing their on-going market positioning and the challenges they thought they were compelled to face”.

Buenos Aires port terminal operator P\&O Terminals also attended the roundtables regularly. Their operations manager - Carlos Barbero - had the following opinion on ALL's participation during the roundtables; "I remember they gave me the impression of being an organization that grew too fast and too much in pursuit of defining their goals and objectives. They were aware of the upcoming trends though did not conceptualize what to do or whom to talk to. However, after attending a number of gatherings and witnessing what others had to say and what some effectively accomplished in terms of JVs and alliances, I believe they did remarkably well. Clearly, it is difficult for me to imagine how they could have possibly taken the right strategies without having had the possibility to benchmark and interact with other firms and people. It is simply unthinkable”.

Multicargo's CEO Carlos Milione had also played the role of witnessing the railways executives' behavior further to having acted as one of the various overseas agents in charge of marketing their services abroad. In his quality of being a supplier of advice and knowledge and attendant to the Corridor roundtable forums he remarked that the regional business context resulted alike for all. However, some were more aware than others of the globalization impact and its possible ramifications. "In the particular case of ALL, it was a great opportunity for them to absorb others' ideas and reengineering proposals regardless of how innovative or challenging they looked. In a threatening scenario like this one, one may easily assume that an organization tends to wait and see rather than jumping into the scene and going through a learning experience against the clock. In this regard I believe these people had the right attitude toward learning, cooperating, and inter-acting with peers and competitors in a really constructive way”.

I had the opportunity to act as consultant for this company while they were attending the roundtable forums. One of the outstanding aspects that describe the way this company has benefited from belonging to and sharing the roundtable outcomes is given by the general upgrade they went through as regards their human resources. ALL soon realized that their marketing and 
sales teams were completely domestic-oriented and that a quick and in-depth human resources internationalization process was badly needed if they pretended to successfully cope with overseas agents, port terminals, and shipping companies.

As such, they identified that the best type of executive they needed to carry out their internationalization strategy was that of a shipping company. In other words marketing and operations executives capable to identify what the international trend in the cargo flow decisionmaking process was, as well as the sales and operations executive having the ability to develop business under an integrated transport mode business (road-ship-rail).

It became apparent to me, as a witness of the process and also being an ocean shipping executive, that a company this big would have never accomplished such a level of awareness of what their weaknesses and needs were, if it were not by the interactions incurred throughout the roundtable forums. The roundtables seemed having proved a fertile ground to capture knowledge, get it internalized and its outcomes channeled toward formulating overseas goals. Without these roundtable interactions, this knowledge would have made little sense.

ALL's President - Eduardo Oliver - admitted needing to reengineer their entire commercial structure which he would have never embarked on if it were not by the experiences and learning process his company went through by attending the roundtables.

Skanska Latin America, a Buenos Aires-based engineering company of Swedish origin, also attended the roundtable forums. They were not a logistic firm but that they attended the forums to better understand the trends some of their clients had to face for them being able to anticipate their future as engineering contractors.

Their regional procurement manager - Martin Gerbino - made the following comments from his non-logistics perspective; "Being ourselves an engineering company and therefore accustomed to dealing with rather complex projects in a very competitive environment, I never expected such a large number and variety of logistic operators interacting and dealing face to face, exchanging information, and to some extent selling themselves on a personal basis to a future potential 
employers. I had here some aspects I did understand and some others I frankly did not - for example why the employers exposed themselves with perhaps those who were their best executives to be head-hunted. On the other hand, I admire the way they intended to get the best from the situation they were going through by doing their best to comprehend others' businesses and market situations. I do not imagine engineering firms doing the same, although must admit that this situation might change if we had to reengineer the whole sector as they had to do".

MTC’s president - Dr. Roberto Cristaldi - had a more cautious view of the role played by this type of participant. He underlined that even though there was an initial sentiment of general skepticism on the potential outcomes a roundtable forum might generate, step by step an apparent anti-business or non-cooperative environment started turning into cooperation and collaboration going from modest to significant. "I personally felt more comfortable to integrate with those groups or study commissions where I came to know somebody from before. I admit that knowing somebody beforehand could have facilitated in the exchange of views and information in my case”.

\subsection{3. - Conclusions on case study \#3}

From the way this case study evolved and by following the comments of the interviewees, as well as my own views from direct participation as a consultant, it becomes apparent that this large regional company found itself isolated while holding a huge potential that needed new goals to be formulated and pursued. Initially it was focused almost entirely on the domestic market to later expand into the region: with no global back-up; having inherited a rather heavy and inefficient managerial and operational structure; lacked a suitable plan or strategic alternative; and needing to increase its load factors to balance an ever-increasing cost base. These were some of the many challenges that seemed to lead this group to join the Corridor.

However, all the above can be considered by no means to be necessary and sufficient conditions to see it as an obvious or natural step joining the forums. The above evidence suggests that ALL comprehended the delicate situation it was about to face as well as it having reduced its scope of action and a great degree of exposure across South America. 
ALL's attitude toward joining the roundtable forums (participate in numerous commissions or CoPs, exchange data and information, and most importantly to try to conceptualize the way other logistic segments were reacting to better face the upcoming globalization) suggests perhaps a daring attitude that was tightly connected with its leadership strategy that the previous case studies did not illustrate.

An active participation in the roundtables and a positive attitude toward exchanging information turned out to be an adequate channel for ALL to reengineer its business almost completely. What transpires from this case study is that it illustrates how the Corridor roundtables facilitated ALL to develop a vision, its leadership, and a positive attitude towards first capturing the value of others' information and to later internalize it to draw a unique global strategy.

What seems to be different in this case study when compared to the first two is that through the knowledge sharing and transfer process, ALL managed to internalize and adopted a different set of dynamics. It was others' exchange of information, views, and opinions that ALL benefited from without having to really enter into any type of formal alliance. Their participation in the roundtables suggests that it was right there where it nurtured its ideas and where it came to learn from and adapt to practices that more international-oriented, better experienced or more talented businesses tended to demonstrate.

Strategies that ALL could only find in the fertile ground and develop at the roundtable forums include: integrating other modes of transport to their existing regional railway network; hiring executives from the maritime segment to better sell their newly multimodal services; appointing overseas agents to exert control over the imports cargo flows; and setting up overseas regional offices for promoting and controlling functions.

It is hard to imagine how a company with such an extensive structure and having inherited a significant burden of managerial problems (typical of a state-owned organization) could have reengineered so quickly and innovatively without being an active member to a Corridor-like entity. Capturing third parties' knowledge, to get their input internalized, and imitating to a great extent what others were doing, seems to well represent lessons to be learned from this case study 


\section{5 - CASE STUDY \# 4: INTRODUCTION}

This case study describes how a former Buenos Aires port operations leading organization decided to sell the business to a large foreign multinational corporation and withdraw from the market to later set up elsewhere in the region and become a competitor to that foreign multinational corporation. Table 6.6 summarizes the data and information concerning this case study.

Table 6.6- Sources of Data for Case Study \# 4.

\begin{tabular}{|l|l|l|l|}
\hline Company & Source & Content & \multicolumn{1}{c|}{ Notes } \\
\hline $\begin{array}{l}\text { MERCOSUR } \\
\text { TRADE }\end{array}$ & $\begin{array}{l}\text { President; Director of } \\
\text { Sao Paulo-based } \\
\text { Sebrae; Director of Sao } \\
\text { Paulo-based Fiesp; } \\
\text { Director of Olam. }\end{array}$ & Corporate strategy. & $\begin{array}{l}\text { Occasional and formal meetings were } \\
\text { held from 02/1998 to 12/2000. Notes } \\
\text { were taken and messages were } \\
\text { exchanged on concepts, processes, } \\
\text { and methodologies on CoPs, } \\
\text { knowledge sharing and stakeholders } \\
\text { integration. Data was validated with } \\
\text { the source by early 2007. }\end{array}$ \\
\hline BAP & $\begin{array}{l}\text { Marketing Manager for } \\
\text { 5 years after holding } \\
\text { various positions at } \\
\text { BAP on traffic, } \\
\text { operations, and quality } \\
\text { control for more than 10 } \\
\text { years. }\end{array}$ & Integration strategy. & $\begin{array}{l}\text { Concepts and personal views and } \\
\text { comments were occasionally gathered } \\
\text { in view of both their secondary } \\
\text { involvement in the forums and their } \\
\text { later set-up of a rail-head within } \\
\text { ATZ. Notes were taken during 1998 } \\
\text { and data was validated in 2006. }\end{array}$ \\
\hline ATZ & $\begin{array}{l}\text { Masiness Development } \\
\text { after holding different } \\
\text { positions within } \\
\text { Murchison's structure } \\
\text { during 20 years. }\end{array}$ & $\begin{array}{l}\text { Search for strategic } \\
\text { partners. }\end{array}$ & $\begin{array}{l}\text { Many informal meetings were held } \\
\text { throughout 1998, 1999, and 2000 in } \\
\text { view of my role at the Corridor and in } \\
\text { my capacity of a potential customer } \\
\text { for ATZ (Zim). Notes were taken at } \\
\text { different intervals and gathered data } \\
\text { was validated by early 2008. }\end{array}$ \\
\hline
\end{tabular}

Bill Murchison was for decades the owner of one of the most traditional and notorious stevedoring company operating in Buenos Aires port right up to the outset of both the globalization and privatization process - which in the case of Argentina arrived almost simultaneously during the late 1980s and early 1990s. Murchison Stevedoring Co. (hereafter referred to as Murchison) accounted for $50 \%$ of both the break-bulk and container segments.

Buenos Aires port terminal is the country’s largest port but by then was neither a modern terminal nor fully segmented one. This port was for long periods of times categorized as a dirty 
port, that is, a port demonstrating a number of inefficiencies among which the most notorious are described as the following characteristics, being:

o Highly unionized and therefore extremely costly in terms of manpower and utilized operational times;

o Federally protected by national legislation and therefore immune to any sort of reform or opening process toward foreign investment;

o Cartelized and therefore facing no price or service differentiation of any kind; and

o Lacking of technological renovation and consequently showing very inefficient in terms of cargo handling and transfer ratios.

Once the port privatization process began with a number of global players arriving in the region and the globalization process was underway, MURCHISON managed not only to remain in the business scene but also to become partner to P\&O PORTS (henceforth referred to as P\&O). P\&O is one of the world's largest port operating conglomerates that was awarded the contract to operate possibly the most strategic terminals of Buenos Aires Port. This partnership agreement established that Murchison should remain as a partner for a limited number of years after which it should sell out its stake to $\mathrm{P} \& \mathrm{O}$ to let $\mathrm{P} \& \mathrm{O}$ control the terminal's full operation. $\mathrm{P} \& \mathrm{O}$ also established a condition by which Murchison could not become a port operator in Argentina in the container segment for a number of years as a natural way to preserve the business $\mathrm{P} \& \mathrm{O}$ had so heavily invested in. Figure 6.4 illustrates how this case study situation evolved. 
Stage 1: Before globalization wave

Stage 2: Argentine port privatization

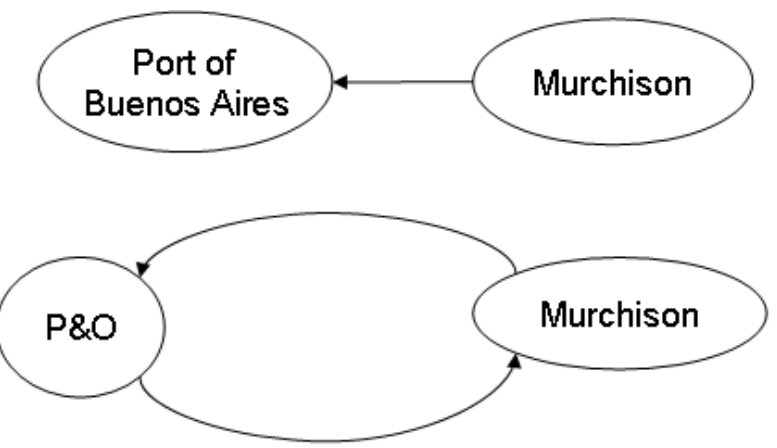

Stage 3: Murchison transformation into ATZ \& move to BA hinterland port supported by SARDIA, ALL, BAP and a range of other regional players

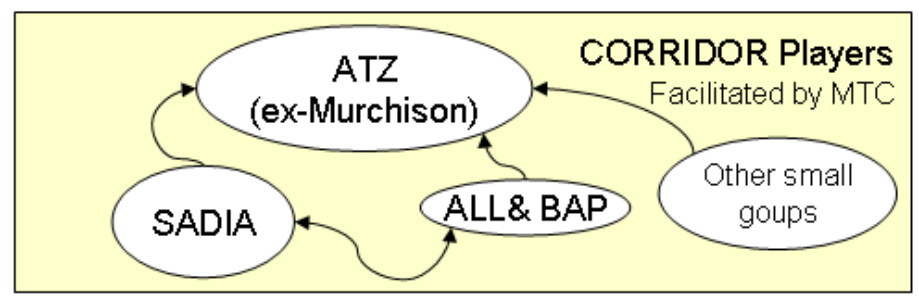

AMERICA LATINA LOGISTICA (ALL), AUTO TERMINAL ZARATE (ATZ) MERCOSUR TRADE CENTRE (MTC), BUENOS AIRES AND PACIFIC RAILWAY (BAP)

Figure 6.4 - Case Study 4 Three Stage Development

In Stage 1 the Port of Buenos Aries was serviced by a number of operators including Murchison. In Stage 2 the port was privatized and Murchison continued its role but dealing with P\&O. Even though Murchison Co. had to ultimately sell out and withdraw from what is been for so long its own territory, to allow a world-class operator to come in and renovate the whole system, they did not disappear at all. Right after selling out they acquired a significant land parcel along the Parana River lying 70 km up-river from Buenos Aires.

A new terminal was built and developed there to meet the automobile industry export and import needs which by then turned out to be a promising business in the long run, given the evergrowing strategic business alliance between Argentina and Brazil. This triggered Stage 3 in which Murchison transformed from a stevedoring company with specialized local, regional and segment-specific knowledge to investor in the new terminal and principal transformation agent in which new port facilities were developed supported by other supply chain members including ALL and BAP (see Case study 3) and Sadia through the active facilitation of the Corridor supported by the MTC. 
Auto Terminal Zarate (ATZ) was by then set up to first operate an automobile terminal to later expand into a $\mathrm{RO} / \mathrm{RO}^{78}$ terminal as well as expanding into the dry bulk segment as a way to generate higher berth occupancy ratios while strategically diversifying their investment. One may assume at this point that while ATZ was becoming capitalized and operational in both the RO/RO and dry bulk segments, it was also getting ready to launch their new full-container terminal from the very moment the ban was over. In order to do so ATZ formalized a JV agreement with a huge foodstuff Brazilian company - Sadia - who despite having no experience in the regional port business foresaw its potential for further development not only in this specific activity, but also in their foodstuff exports and distribution system throughout Argentina. This meant that more than one third of their exports would later become the main destination for Sadia's products.

This case was chosen to be included to illustrate how the Corridor facilitated not only a unique example of knowledge exchange between potential and actual rivals but also how a business opportunity was opened through this mechanism.

The next section will address the way these two players actually interacted and what they saw and learned from each other's opinions on other stakeholders.

\subsection{1. -Buenos Aires' Hinterland potential: A matter of knowledge and vision}

It becomes clear at this point the Murchison's expertise in the port business was born and developed much earlier than the Corridor had been established. Having decided to invest and operate a new terminal specialized in those non-conflicting segments with their former partners $\mathrm{P} \& \mathrm{O}$, while nourishing the concept of a nearby new-brand container terminal, are aspects that cannot be attributed to the Corridor.

These are aspects that are naturally based on the knowledge and vision of the company's owner and managers. It was precisely this level of knowledge that allowed Bill Murchison's firm to first partner with a global player, then to later expand into other segments and challenge $\mathrm{P} \& \mathrm{O}$ on their

\footnotetext{
${ }^{78}$ RO/RO: roll-on/roll-off facilities are those where self-propelled on-wheels cargoes are operated. Not only automobiles but also trucks, road-making machinery, side-booms, etc.
} 
own terms. What remains to be ascertained is the identity of the party that actually benefited most from the original JV.

Was it P\&O PORTS gaining from Murchison in that the former gained the regional knowledge of uses and customs of the trade through a process which let them explore and perhaps capture their ideas and concepts that they managed to develop throughout the years they were bound in the JV? Or did Murchison gain from P\&O in terms of global vision, technology, management, and operations? While $\mathrm{P} \& \mathrm{O}$ brought to the region very sophisticated information systems to efficiently design loading and discharge pre-planning operations, global positioning system (GPS) tracking devices for chassis and containers, container freight station (CFS) management, and port-designed handling and loading equipment. Murchison contributed by: making available their long-standing relationship with customers (shipping companies and shippers); having an efficient union management; having effective coordination with all the multimodal players along with a deep understanding of the political context that for long periods of time led the country's economy to face stop-and-go economic processes.

One might possibly infer that both have benefited from each other though from different angles. However, Buenos Aires' hinterland still today hides an enormous potential in terms of port business development as a result of an historic dependence on Buenos Aires for most of the country's foreign trade. Similarly, the northeastern strap of Brazil is not serviced by the global shipping companies or world-class port operators. Traditional or regional knowledge therefore is of decisive importance for both the regional companies to reorganize and compete, and the global firms to avoid or limit local competition.

It becomes apparent that besides the very particular characteristics that the Buenos Aires' hinterland might hold, which on first sight one may infer is at the advantage of the Argentine firm, both companies held a significant amount of knowledge to share and transfer to each other. Knowledge viscosity levels and absorption difficulties remained low for both parties given their respective expertise in the field. 
Unlike the previous case study where the railway company had everything to benefit from the Corridor, the situation seemed here to have been quite the opposite. Knowledge, leadership, change, vision, cooperation, and collaboration, all played a fundamental role to make their contribution before the Corridor roundtable forums became established.

While P\&O focused on both upgrading and reengineering their business by installing the latest port handling technology and infrastructure aiming at achieving larger economies of scale and gaining competitive advantage, ATZ capitalized on all those agro-industries that for decades had been asking for a competitive alternative port to Buenos Aires - a port which they have always regarded by a wide array of hinterland industries as being expensive, inefficient, and corrupt.

In this regard I had the opportunity to assess ATZ's SWOT analysis where perhaps one of the outstanding features was precisely the anger and frustration a rather large number of industries located in the hinterland had stated against the dominant and centralized role that Buenos Aires port had played for decades. A search for revenge on the part of a wide array of industries was duly capitalized upon by a company that no long had been profiting from such a centralization scheme. What these industries failed to realize, and did not want to experiment with, was that $\mathrm{P} \& \mathrm{O}$ had come to reengineer the whole port system along with other global players who undertook the same process in the nearby terminals.

The growth of a number of Chilean ports outside the main Valparaiso port clearly illustrates the potential of Buenos Aires hinterland as it can be attributed to the above mentioned Argentine industries that by all means looked for operational alternatives in their permanent search for both remaining competitive in the international markets and keeping their imports of raw material and equipment as inexpensive as possible. 


\subsection{2. - The corridor as a facilitator}

Both ATZ (ex Murchison) and P\&O Ports knew perfectly well what they were after and what their possibilities were to successfully develop their own business either prior to or during the nurturing of the Corridor. $\mathrm{P} \& \mathrm{O}$ had shown themselves indifferent and even somehow arrogant during the roundtable forums - possibly based on their size, financial resources, and a formidable global structure.

ATZ, on the other hand and even though it could not be compared to P\&O in terms of size or resources, was the owner of a very deep regional knowledge arising out of long years of having been in touch with worldwide ship-owners in their capacity of being the Buenos Aires port main stevedoring firm. Such an exercise supplied them with the necessary global vision to anticipate how the globalization would modify the way to do business in the region as well as to leaving their port knowledge and regional expertise untouched.

Sadia was also a member to the Corridor despite having no direct intervention on the logistic subjects that were exposed and analyzed during the regional sessions. Very much alike Argentina-based Refinerias De Maiz, their main task was to make sure their various logistic channels linking Brazil with Argentina remained open and efficiently run.

MTC not only represented a number of companies that effectively joined and participated in the Corridor, either as primary or secondary stakeholders, but also some specific trade and industrial federations based in Sao Paulo, Rio do Janeriro, and Porto Alegre, Brazil. MTC’s President Dr. Roberto Cristaldi described Sadia as a company that despite having played a secondary role within the Corridor, became more and more involved as the globalization was transforming the regional business scenario: “it was a company more interested in establishing a political presence rather than actively participating in the various commissions that focused on a number of topics that were not of their direct interest. However, once the barging companies started being absorbed by some ocean shipping companies while the port terminals changed ownership along with the by then existing coastal feeding services, they must apparently have seen their transportation and distribution channels threatened". 
BAP's marketing manager's opinion tends to concur with the above opinion in that SADIA was regarded as a secondary stakeholder until reaching the stage when it allied with ATZ. From then on it changed its attitude and started interacting and exchanging information in the various CoPs it had joined. Sadia had approached BAP together with Murchison to establish a fully-dedicated operational rail-head capable of storing and distributing from nearby their terminals into the centre and centre-west region of the country.

ATZ's business development manager - Maria Quiroga - had the function to look for strategic alliances that might lead her company to better cope with the huge export and import potential the interior held for so long. To that purpose the Corridor seems to have played a suitable role as the most qualified and reliable partners at hand; "we actually never attended the roundtable forums in search of knowledge, people, or concepts that we might later adapt to or make good use of, but that we rather concentrated on interacting with potential allies that could help us challenge the dominant position we lost to $P \& O$ in Buenos Aires. We always knew to a great extent what the globalization was like and the effects it could bring attached. We had the necessary skills to adapt to that situation and the Corridor resulted being the ideal place for us to select the right partners". When asked about the attitude taken by P\&O Ports when attending both the roundtables she made clear their impression on P\&O's indifference to the on-going activities. "It was evident for $P \& O$ that they suspected we were getting ready to strike back as practically all attendees or primary stakeholders were engaged in making their own arrangements in terms of merging, reengineering, downsizing, joint-venturing or whatever. And we were not the exception to that rule. Besides, they had both the size and structure that made their participation more political than practical as they were not in need to restructure, reengineer or identifying a potential partner to expand into the region.”

When asked about which, in her opinion, where the outcomes sought by both ATZ and P\&O at the forums she stated that for ATZ the roundtables provided them with a very unique opportunity to interact, exchange views and opinions, and identify potential allies to help them expand into the multimodal business within their hinterland as well as benchmarking opportunities beyond their hinterland. The JV with Sadia demonstrates that the Corridor roundtable forums proved to be strategic for ATZ's development plans. 
As far as $\mathrm{P} \& \mathrm{O}$ was concerned she believed their main interest was to show commitment with the Corridor's original idea and fundamental concepts before their customers (shipping lines and major shippers), as well as benchmark with other terminals to find out and compare what they were doing and to present themselves before potential new customers who happened to be primary stakeholders of the Corridor.

As a direct participant I had the opportunity to witness the existing close relationship between Sadia and ALL and the quite open way they dealt with one another in view of their long existing relationship in Brazil that later projected into Argentina. The railway company used to carry many of Sadia's products across the vast Brazilian geography and get them door-to-door delivered by utilizing a large number of both trucking contractors and its own fleet, while Sadia's competitive logistics within Brazil was due to ALL’s wide network and relative lower operational costs for long distances.

It is been my perception that as well as the previous business relationship maintained by these two firms, a certain degree of cultural affinity may have helped the two companies understanding each other more rapidly than if had they been of different nationality. However, when the latter situation actually arose between an Argentine and Brazilian firm, the most common of the situations, by no means tended to hinder the interaction and its consequent knowledge exchange from happening but that it tended to slow it down at the beginning to later catch up with those of a closer affinity, suggesting the existence of fairly low cultural barriers amongst the Corridor players.

MTC’s Dr. Cristaldi highlighted the salient point that the main cultural difference he perceived during all those years of attending the forums and dealing with those professional federations he represents. He observed that cultural difference was not evident from codes, values, or more daily aspects such as feeding habits or life styles.” It was only a matter of timing by which Brazilians looked more reflective than their Argentine counterparts who tended to be more resolute on the decision-making process though perhaps less strategic. Other than that, I see no major barriers as far as culture is concerned". 
None of the interviewees showed discrepancies with the above mentioned opinion nor defined the term culture as a real inhibitor or barrier to surpass, but that highlighted the existing high degree of integration among the Corridor members long before the Corridor was established. The next section will address the case study conclusions by focusing on the role taken by the two main players.

\subsection{3. - Case study conclusions}

The evolution of this case study along with the comments and opinions gathered from the interviews along with my own view, suggest that both ATZ and Sadia brought with them a considerable level of both knowledge and leadership in their respective fields. ATZ was a successful port terminal developer and operator in Buenos Aires port that right after selling out their stake to a global leading company, set up a new and entire port terminal to challenge their old partners. Sadia was a leading Brazilian foodstuff producer and exporter of both regional and international reach holding an increasing market position in Argentina.

None of the two companies had apparently joined the Corridor to pursue the objectives they later set, but that initially played a kind of political presence with the intention to sound out potential synergies with a limited number of contractors (ATZ), and to control or reassure their logistic channels were kept operational and competitive (Sadia). However, the Corridor seems having facilitated these companies achieving higher benefits from their association through roundtables. ATZ, for example, found a solid partner to carry on with their expansion strategy while Sadia also found the ideal partner to consolidate their presence into what was becoming their main exporting market.

The Corridor roundtables facilitated these companies to get together as well as exchange vital data and information that later led to a successful JV on a win-win basis. Regardless of the JV partners and their respective leadership and visions on the upcoming transformations the region was going to face, the strategic outcomes arising out of their participation on the roundtables seems having a direct link with their own businesses’ strategic positioning. 
Even though the two corporations joined the Corridor with different objectives, both found a fertile ground to develop contacts and synergies to grow beyond their primary goals and without coming across with substantial cultural barriers. Both tacit and explicit knowledge sharing and transfer seems having been fluid between the parties. The relative easiness with which the contacts were made and developed in the right direction with respect to the main players and a great number of logistic contractors, does suggest that the two main actors must have been very much aware of the upcoming threats and that an urgent transformation was needed, the business context pressure being of paramount importance.

Perhaps being both well established companies in South America, they also knew that a crisis might bring about opportunities to take the place others would leave open, especially by taking advantage from the apparent cultural advantage outsiders could not count on. The relative homogeneity with which the various players approached the Corridor together with a rapidly changing business environment, seems to have played a key role in making knowledge sharing and transfer take place, leadership to emerge, and cultural barriers to be lowered to allow associations and alliances of various types to be shaped.

Thus, the Corridor looks for this particular case study like having played an essential role not only in making the two corporations contact each other, but also in having created a knowledge exchange-oriented forum for them to explore each other and nurture the germ of strategic corporate collaboration and cooperation, possibly witnessing the way others had undertaken this attitude. This case study suggests that the roundtables forums and their respective CoPs were crucial to letting leadership, knowledge, cooperation, and collaboration interact and bring change and innovation. This also allowed synergies to play a new dimension in leading to strategic alliances which otherwise would have probably taken too long to happen.

\section{6. - CHAPTER SUMMARY}

This chapter presented 4 case studies that involved the Corridor roundtable discussions and associated CoPs for companies forming associations and business transformations. These case studies were selected to illustrate how change may be seen to have been instigated as a 'project' or at least program of activities that: 1 ) brings together potential independent actors with a 
common interest and rationale to collaborate; 2) explains the rationale and motivation and process of the Corridor's involvement and impact in facilitating this transfer; and 3) focuses on the role of knowledge transfer, trust and commitment building, and leadership in implementing these transformations.

These four case studies are of a business network development and management type identified by (Artto and Kujala, 2008) as falling within a management of a business network type of PM study. In fact they extend that emerging field of study by providing insights into the genesis of the formation of a business network as well as a radical transformation of the way those businesses dealt with severe problems they faced.

The four case studies show a number of similarities as well as differences that vary according to the type of segment dealt with, the market positioning of the involved companies, their global structure, size, expertise, and a number of other variables. There are a number of variables that happen to be common to all of them, supporting the idea that the Corridor roundtable forums have been instrumental toward allowing cooperation, collaboration, knowledge, and leadership turn up and produce realistic alternatives before what was regarded a global trend and an uncertain business context. These can be summarized as follows:

1. The relative easiness or willingness to communicate and interact on the part of a vast majority of the stakeholders, either primary or secondary, suggests that a stronger pressure was exerted on the region by a threatening uncertain business context than from an implicit level of cooperation amongst the participants. This immediately present driving motivator seems to have offered the Corridor as looking like a promising and appropriate entity to channel their worries, anxieties, and corporate ideas toward a productive and sustainable end. Typical variables often found on the CoPs such as rivalry, antipathy, envy, jealousness, information ambiguity, or cultural barriers were not identified as real knowledge detractors.

2. Leadership in terms of gaining trust, commitment and shaping a vision for a preferred future seems to have a direct link with those firms showing previous overseas experience from business contexts being more cultural diverse and operational constrained. Not only did the Corridor leaders' extrapolation of knowledge and experience turn out to be 
contagious for others Corridor members (followers) to undertake a more active interaction with peers and competitors, but also their pro-active attitude toward searching for potential allies and transformations helped them achieve larger economies of scale and gain competitive advantage.

3. Immediate followers followed the example of the truly global firms but held a lesser relative degree of commitment to the region though showing a certain level of global reach and operations. There also seems to have been an influencing factor to either become a leader or a follower. Leaders tended to be actually holders of a greater relative influence on the cargo-routing decision making along with their relative market positioning at a given point in time. A game seems to have evolved where members try to ascertain who most influenced the relative market forces in order to define leaders and followers. This was not so much a matter of a corporation's size, structure, or deployed assets across a predetermined geography.

4. Openness to interact and consequently share and transfer knowledge within the forums seemed dependent more on a company's relative strengths and competitive position at a given point, and not so much on what one might assume related to cultural or nationality aspects. Those firms enjoying perhaps a stronger perception of their present market positioning and/or what the future might bring in terms of business opportunities or market structure transformations tended to be knowledge facilitators. Such phenomena may have impacted contagiously on others who by then had been seen only as followers or somehow indifferent to the on-going business context.

5. Similarly, the interaction and communication process (and the driving and proclaimed voluntary nature of the various $\mathrm{CoPs}$ ) found participants' actual motivation more impacted by the rapidly mutating business context than on the executives' own initiative in their search for answers. This suggests that the region had to reach a critical tipping point at which participants' own market structure and business development were at stake for them to react and support these kinds of professional interactions. Once again, an extreme business environment situation along with the upcoming globalization seemed being the driving force for many actors to join the Corridor and support their functioning.

6. The existing traditional knowledge on the part of the regional companies along with the cultural asymmetry between the regional players and those from abroad, tended to help the 
regional players to accrue knowledge value in their own companies. Even though the level of traditional knowledge varied according to the geographic scope and type of logistic services in question, it did help in creating a given level of dependence or knowledge market which many regional operators tended to conceptualize and profit from. Additionally, the existing cultural gaps between those extra-regional operators (especially the newcomers) and the regional firms also acted as a kind of value accumulation on the part of the latter. This helped regional companies to build a level of dependence that outsiders had to recognize as a vulnerability they had to address to be able to expand into the region. The Corridor roundtable forums reinforced the regional players' awareness of these two powerful variables that eventually played into their favor, as well as on the vision and minds of the outsiders who foresaw the need to include these variables into their plans.

7. The globalization in itself and the Corridor members' pro-active attitude did have an impact on those laggard firms in that it helped them comprehend and internalize the imperative necessity to update and restructure to face the future. This process suggests that the roundtables effectively influenced those who owing to their relative lesser economies of scale or market positioning had not taken any leadership position nor opened up toward a fruitful potential interaction.

8. Cooperation and collaboration showed little inhibition or impediments among complementing companies. These saw in one another a potential business opportunity to adapt and reengineer to cope with uncertainty. Once more, the roundtables seemed to have helped these companies gain a winning awareness of their joint potential and of their need to reengineer to better utilize their assets and management.

9. Lack of focus was an element that became apparent both in those state-owned companies, where goals and motivation were absent variables, and those corporations holding little or none internationalization. In both cases, the Corridor seems to have played a role of paramount importance not only in letting knowledge sharing and transfer taking place, but also in somehow redefining the leader-follower relationship. The creation of a knowledge market, where precise knowledge was an appreciated commodity, eventually helped triggering the appearance of other crucial variables such as: cooperation and collaboration; 
leadership; and change and innovation. These resulted in a renewed business and benefits results focus.

10. Regional cultural asymmetries tended to highlight some differences in styles and timing. However, and possibly because of the upcoming business context transformation, they were never a real barrier for the players to reengineer other than to perhaps slow down the interaction process. The Corridor seemed to have highlighted first the cultural asymmetries between the regional and the extra-regional players to later dissolve those intra-regional differences in favor of a rapid and innovative adaptation process.

11. The active commitment and participation on the part of the outsiders did have a driving influence on many regional firms in that they saw themselves compelled to back the knowledge sharing and transfer mechanism. They not only attended Corridor roundtables to learn and capitalize from others, but also they came to realize they had also became part of the forums in that they were needed as someone else's strategy to enter into a JV, agreement or partnership.

12. Knowledge stickiness became apparent in those non logistic-oriented corporations holding non common objectives and those logistic complementing companies of relative lesser market share and influence. Some of them turned out to be just observers while others were regarded as laggard players. In all cases, Corridor-born synergies played a significant role in improving this situation. Corridor officers were active brokers and monitors of the process and these activities became a recurrent need to allow firms both to profit from and to contribute to the roundtables. This suggests that the argument of a threatening business context may not necessarily drive companies to take action to share and contribute knowledge but that it might also depend more on each company's history and current competitive positioning.

13. A face-to-face interaction seems to have been the preferred channel to let tacit knowledge flow and to bridge apparent cultural asymmetries among the leading primary stakeholders. This process was soon imitated by followers, both primary and secondary stakeholders, to later expand (though to a limit) to those classified as laggards or showing indifference to a system they just adhered to for political reasons.

14. Indifference was a factor that despite having played a negative role during the initial stage of the Corridor life cycle, turned out to be partly offset by the roundtable attendants' own 
dynamics that turned on-going indifference into productive synergies leading to a deep involvement of some corporations. This suggests that even though some companies had shown indifference or lack of initiative, they ended up by setting up alliances or agreements beyond their own fields of activity and possibly their expectations too.

There were some aspects that did not prove pivotal in hindering Corridor players' knowledge transfer between and leadership from emerging and spreading more widely amongst Corridor players. However, these aspects do deserve further brokerage efforts through the Corridor for it to produce greater regional benefit. These aspects include participant indifference, laggards, potential cultural asymmetries, corporate cautiousness, and lack of focus. 


\section{BARGE TRANSPORTATION SUB-CASE STUDY}

\section{1 - INTRODUCTION}

This chapter relates to the waterways supply market evolution and the way the project stakeholders had to deal with the particular business context it faced to overcome (either in a purely transactional management mode or transformational style) the many constraints that emerged. The response by the players to the business environment, particularly radical changes in focus on supply chain management towards value chain management (which exemplifies the Winter et al (2006) research direction 3) seemed to have played a more decisive role than the management styles deployed by the different actors. The specific project focused upon in this study comprised the construction and operation of fully dedicated barge convoys to carry iron ore supplies from Brazil to Argentina commencing in 2003 and phased over six-years. The first phase comprised development and acceptance of an innovative logistics design solution and its realisation through the construction of new barges and leasing of existing barges. The project had in-built replication possibilities to roll out a series of similar projects.

As with the previous case studies, this chapter exposes a similar rationale governing issues of collaboration, cooperation, knowledge sharing and transfer, leadership, and culture. It also emphasizes the way the globalization process impacted the region and somehow facilitated the creation of the Corridor to allow different players to network and exchange knowledge. However, this case study differs from the previous chapter's four case studies in that a different type of traditional tacit knowledge plays a specifically critical role in making this river transportation transformation project a reality. The way that this traditional knowledge resource was shared and used is shown to be a crucial element of the transformation. Also, the geographical setting where this project is put into practice, along with its operational complexity, turns it into a unique case study.

Another important difference is given by the participation of a major multinational company that has no business or specific interest in any logistic segment. However, this firm happens to be a primary stakeholder in a supply chain that involves Corridor logistics firms. All the other 
stakeholders are, to a greater or lesser extent, logistic-related firms doing business in the region each of them possessing a highly valued quota of traditional knowledge..

This case study will demonstrate how a supply chain management (SCM) problem triggered a business transformation with a more complex and demanding scale than was the case with case studies 1-4. The manner in which this SCM transformation was carried out further illustrates and helps explain how the Corridor was pivotal in managing this radical change management project.

It continues with a description of the project stakeholders by highlighting the role played by those regarded as instrumental to the project, and addresses in a separate section the various negotiation steps it took for the project to kick off. It ends with the actual contribution of the Corridor to the project completion and the chapter's final conclusions. Table 7.1 describes the following sources of information.

Table 7.1: Sources of information.

\begin{tabular}{|c|c|c|c|}
\hline Company & Source & Content & Notes \\
\hline Southmark Logistics S.A. & $\begin{array}{l}\text { Project Manager with } \\
\text { an extensive project } \\
\text { logistic experience } \\
\text { acquired throughout } \\
30 \text { years of regional } \\
\text { experience. }\end{array}$ & $\begin{array}{l}\text { Business } \\
\text { networking strategy } \\
\text { and personal } \\
\text { insights on } \\
\text { knowledge sharing. }\end{array}$ & $\begin{array}{l}\text { Informal chats, gatherings and } \\
\text { lunches in view of working } \\
\text { together for the same company } \\
\text { and based on a long-lasting } \\
\text { knowledge of this person's } \\
\text { feelings, views, and } \\
\text { professional opinions and } \\
\text { understanding of the region. }\end{array}$ \\
\hline Mercosur Trade Center & $\begin{array}{l}\text { President; Director of } \\
\text { Sao Paulo-based } \\
\text { Sebrae; Director of } \\
\text { Sao Paulo-based } \\
\text { Fiesp; Director of } \\
\text { Olam. }\end{array}$ & $\begin{array}{l}\text { Role played by } \\
\text { traditional } \\
\text { knowledge and } \\
\text { leadership styles on } \\
\text { the part of the } \\
\text { project } \\
\text { stakeholders. }\end{array}$ & $\begin{array}{l}\text { Occasional and formal } \\
\text { meetings were held from } \\
02 / 2004 \text { to } 12 / 2005 \text {. Notes } \\
\text { were taken and messages were } \\
\text { exchanged on concepts, } \\
\text { processes, and methodologies } \\
\text { on knowledge sharing and } \\
\text { stakeholders collaboration } \\
\text { process. Data was validated } \\
\text { with the source by early } 2007 .\end{array}$ \\
\hline Navisur & $\begin{array}{l}\text { Buenos Aires-based } \\
\text { ship-agent for the } \\
\text { Paraguayan barge } \\
\text { operator holding an } \\
\text { extensive operational } \\
\text { experience of more } \\
\text { than } 20 \text { years in South } \\
\text { American fluvial } \\
\text { operations. }\end{array}$ & $\begin{array}{l}\text { The Corridor as an } \\
\text { adequate arena for } \\
\text { complex project } \\
\text { development }\end{array}$ & $\begin{array}{l}\text { Regular short, informal and } \\
\text { frank conversations normally } \\
\text { held during lunch time and } \\
\text { while attending the roundtable } \\
\text { forums during } 2005 \text {. } \\
\text { Occasional exchange of views } \\
\text { and professional opinions were } \\
\text { made when developing the } \\
\text { logistic design. Content and } \\
\text { conceptual aspects were }\end{array}$ \\
\hline
\end{tabular}




\begin{tabular}{|l|l|l|l|}
\hline & & & $\begin{array}{l}\text { revised and checked with the } \\
\text { source by late 2007. }\end{array}$ \\
\hline Wachovia & $\begin{array}{l}\text { Miami-based project } \\
\text { director for the } \\
\text { Americas with a solid } \\
\text { experience of more } \\
\text { than 30 years } \\
\text { developing logistic } \\
\text { projects in ports, } \\
\text { shipping, and } \\
\text { multimodal integration } \\
\text { projects. }\end{array}$ & $\begin{array}{l}\text { Business strategy } \\
\text { across the } \\
\text { Americas. } \\
\text { Managing Director. } \\
\text { breakfast where an informal } \\
\text { chat was held and a few short } \\
\text { and project focused comments } \\
\text { produced. Concepts were } \\
\text { checked with the source and } \\
\text { validated during 2007. }\end{array}$ \\
\hline Southmark Logistics S.A. & $\begin{array}{l}\text { Musiness developer } \\
\text { and task } \\
\text { coordinator. }\end{array}$ & $\begin{array}{l}\text { A complete version of the } \\
\text { contract in Spanish language } \\
\text { can be obtained by contacting } \\
\text { info@southlog.com }\end{array}$ \\
\hline
\end{tabular}

\section{2 - INTRODUCTION TO CASE STUDY 5}

Iron ore and magnesium reserves all over Bolivia's Mutun region are estimated to reach 40 billion tons according to recent prospects carried out in the region ${ }^{79}$, without even considering those located in Brazil's Matto Grosso Do Sul area, which turns out to be another large source of supply of these key raw material utilized in the steel industry. Mutun's iron ore deposits are considered to be the world's largest ${ }^{80}$.

While Brazil is a significant producer and exporter of this strategic mineral, Argentina lacks it and is therefore increasingly dependent on Brazil as a supply-source to manufacture steel tubes, rolls, and slabs to be exported all over the world. Traditionally, this role is been mostly dominated by a single industrial Argentine multinational group whose supply sources were spread all over the globe, as a way to avoid too much concentration that might negatively impact on the production cycle should anything go wrong on the supply chain. Thus, and beyond any quality assessment on the product itself, their supply sources were utilized as per needed and by making good use of the opportunity cost concept.

About one quarter of the supply needs was consequently allocated to each of the following historical supply origins:

$>$ Matto Grosso Do Sul - Brazil: this source of supply was rated as supplying a good product quality vis-à-vis a poor logistic performance. Its main advantage - product quality - often

\footnotetext{
${ }^{79}$ Will Evo stick?". Mines and Communities Website. Retrieved on 2008-03-29

80 "Serrania Mutun, Chiquitos Province, Santa Cruz Department, Bolivia". mindat.org. Retrieved on 2006-10-20.
} 
turned out to be of relatively good value as compared to alternative that involved barge convoys sailing 2,400 km along the Parana-Paraguay waterways across 4 countries in order to reach final destination, where on time and on budget performance often proved to be just an aspiration. Therefore, the steel plant's critical stocks often had to increase to offset occasional shortages of iron ore which would ultimately make other sources gain a relative higher share of the required supply.

$>$ Newcastle and Fremantle - Australia: a lower relative quality iron ore was regularly supplied on ocean-going vessels in shipments of up to 40,000 tons on "Panamax" type ships of 60,000 tons of deadweight. Even though these vessels held more capacity and were therefore underutilized owing to the on-going River Plate draft limitations (that are explained later in this chapter) - this resulted in higher ocean freight rates per ton. However this still turned out useful in offsetting any supply shortfalls arising out of the waterways' recurrent logistic bottlenecks and service interruptions. A full shipment from Australia generally supplies 40 to 45,000 tons with a 30 days sailing time, whereas a full convoy on the waterways often supplied a tonnage ranging from 18 to 30,000 tons (depending on seasonal draft) and a sailing time ranging from 15 to 60 days - depending on a number of operational variables. It was clear by then that the waterways' variability in reliability of supply was high and this certainty resulted in both performance outcomes in terms of low loading volumes and poor sailing times - without even considering port congestions at the Brazilian river terminals as well as a modest loading speed. River draft variability was subject to a dynamic seasonality that made planning risky and tricky. Constraints related to the existing regulatory framework as regards convoy fractioning ${ }^{81}$, night navigation ban, and safety controls also played its role in tightening the operational window even further.

> Mobile, USG - United States: although the product was of high quality and the sailing time and/or certainty of physical delivery resulted much better than the two options previously mentioned, the existing draft limitations on the River Plate limited the size of the vessels to the underutilized "Panamax" type and therefore logistics economies of scale were only partly accomplished. Consequently, this supply source was in being very similar to that of the

\footnotetext{
${ }^{81}$ This means splitting or separating cargos into smaller loads to lower the barge draft (depth from the waterline to the underside of the barge) or to cope with narrow unobstructed channels. Fractioning is critical where the barge is travelling over rocky and shallow waters or through narrow navigable channels within the river. Fractioning cargoes produce inefficiencies in carrying capacities but are necessary to avoid grounding risks.
} 
Australian option in terms of operations and productivity. However, Mobile turned out to be an efficient supply source capable of quickly offsetting a shortfall of any other sources owing to both its relative geographical proximity and ample ship tonnage availability (unlike far away located Australian ports and problematic river services). These variables were reinforced by an existing easy access to the commercial or negotiating channels to reach both shipping companies and qualified shipbrokers capable of giving the right response at shortnotice. However, this supply source also started allocating an increasing degree of preference to those purchasing orders coming from China and India - two markets that represented markets of much larger volumes and higher prices than the River Plate. Australian suppliers also showed the same tendency with respect to the Chinese market.

> Sepetiba, Tubarao, Carajas - North Eastern Brazil, product quality here was not the best though both product delivery response by the producing mines and sailing times offered a result being close to optimal. However, continuous labor strikes, unannounced port lock-outs, and the ever-existing draft limit on the River Plate - affecting all ocean sources alike restricted this source of supply to no more than one fourth, and often even less than the total required.

It is interesting to point out that all the players at this stage, both on the raw material supplying side and the receiving party, were large, well-established, and powerful multinationals financed with an interesting array of capital from: Argentina / Australia / Italy / Brazil / UK / USA. Therefore, these corporations' markets represented the world itself whereas the River Plate market was just a marginal destination of a much lesser relative weight when compared to potential buyers in the Asia-Pacific region. On the river logistic side, the waterways were dominated by a number of traditional barge operators displaying great variability as regards barge and tugboat technology, management, operational versatility, financial standing, and reputation, all which led the Argentine steel company to exploit those asymmetries by playing one river operator off against another as a way to negotiate lower freight rates and short-term contracts in their favor. 
Therefore, the Argentine group historically supplied their needs of iron ore from four regular sources across the world by combining a number of variables which they always tried to optimize according to the on-going contextual circumstances:

- $\quad$ Level of critical stock to avoid production shutdown;

- Total transit times from supply source to destination’

- $\quad$ Mix of freight rate levels and type of services;

- $\quad$ Mix of product quality to allow a competitive end product;

- $\quad$ Draft limitations and its ratio to the critical stock level.; and

- Operational constraints of any nature, such as strikes, lock-outs, stranding, collisions, etc.

Ocean shipping operators supplied their vessels in a normal fashion within a context of relative stability and predictability. Time-charter rates varied within a range that was considered reasonable and ship-owners did not hesitate to engage into one-year contracts for carrying iron ore from Australia, the US gulf, or northeastern Brazil in view of the return trip being often backed-up by grain shipments from the River Plate up to the origins’ nearby locations. Both ships hiring rates and bunker prices - two critical interrelated cost factors for any shipping company remained under control throughout most of the 1990s.

All the above might be considered as the normal on-going circumstances that historically had governed the supply-chain of this organization with respect to a commodity or raw material. These were not seen as a strategic factor in a supply chain but rather a commonplace input. Consequently, the supply-chain management of this group could be regarded as sensible and reasonable within an international context of relative stability where suppliers did not fail to honor their delivery commitments; no supply source was a major or dominant player; the everexisting operational constraints were always, to a lesser or greater extent, under control; demand for steel products and its price levels were relatively stable; logistic suppliers were manifold; and logistic bottle necks of any kind were mostly manageable.

However this business scenario, being of relative certainty with no drastic or major changes during the 1990s, suddenly started to experience a structural shift for the following reasons: 
1. China's economy started to demand an increasing volume of various commodities, among which two were to be critical for the steel making firm's supply-chain (iron ore and soy). An increasing demand of iron ore made this firm's historical suppliers in Australia shifting their preferences and supply to Chinese buyers on the basis of higher prices and long-term commitments based on price adjustments. China's proximity and its shorter financial cycle also played its part. China's growing demand for soy products - of which not only Argentina but also Brazil, Paraguay, and Bolivia were by then (and still are) large exporters - changed the commercial priorities of the barge operators in the waterways. The barge operators gave up carrying iron ore to load a much better profit-yielding product (soy and its byproducts) which despite being seasonal offered them the typical commercial advantages of a product mix that was heavily demanded on the other side of the world. Even though the barge operators did not disappear and kept carrying iron ore along the waterways'2,400 km of waterways, their commitment, compliance, and reliability left much to be desired. Service informality then loomed large and became a commonplace.

2. Even though the Gulf War triggered oil prices soaring in the early 1990s and introduced a certain element of uncertainty on the river and shipping markets worldwide, this crisis alone neither impacted on the market structure of the shipping companies nor on that of the river barge operators. Bunker costs were duly adjusted according to their respective bunker adjustment clauses and suppliers kept carrying iron ore in a regular fashion. However, the situation in 2004 seemed different with different causes with respect to oil prices despite these having also soared in a significant way and within a context of growing global uncertainty. The difference can be explained by the pressure China's economic expansion exerted a critical and drastic business scenario change through:

1. China becoming the first priority as a long-term customer;

2. Ship tonnage supply being mostly committed to China;

3. River tonnage supply giving preference to supply China; and

4. Ocean time-charter rates and river freight rates tending to increase sharply owing

a. to supply shortage and excessively high bunker rates which made operators retreat and cancel new-buildings orders; and 
b. Short-distance sailing being preferred to long-distance sailing by the shipping companies as a way to reduce their financial exposure to a global price of USD 50 per barrel time-charter rates.

From my own company and the steel-producing company's business intelligence sources, it is clear that steel prices also exhibited a growing trend throughout 2004, which if compared to the previous year's average of USD 300/ton, resulted in an increase of more than $70 \%$. This evolution made the steel-producing company increase their physical assets by investing in an entire new plant to cope with the growing global demand, while the fluctuations in the oil price made barge operators becoming cautious by not engaging in new buildings.

\section{3 - SUPPLY MARKET STRUCTURE}

The waterways market linking Brazil, Argentina, Bolivia, Paraguay, and Uruguay were until the 1990s, mostly dominated by both Argentine and Paraguayan barge companies with significant capital investment in barges, tugboats, river terminals, handling equipment, and specialized management. This was historically so when the currency parity of the Argentine Peso to the US Dollar became 1 to 1 , at which time the country's economy started to open to the world.

Thus, Argentina received a significant flow of foreign direct investment in practically every infrastructure segment or economic activity of interest such as railways, ports, electricity, hydrocarbons, water, grains, foodstuff, mining, and power generation. Both private and public national firms saw a bright opportunity for capital investment thanks to the high valuation that their assets enjoyed, based on the existing 1:1 USD to Argentine Peso currency parity. Barging companies were no exception and a deep and rapid process of capital concentration took place, first from the USA and later from Chile - two aggressive investors in the country at that time.

Traditional barge operators with interests all along the waterways started to retreat, sell out their assets, and either reengineer their businesses or disappear from the market. Most of the few existing Paraguayan operators sold off their companies and gave up the river business too. By the end of the 1990s the market was mainly operated by a strong and financially solid (though unskilled) foreign barge company whose only competitors were a bunch of small independent 
persistent operators from both Argentina and Paraguay. Figure 7.1 illustrates the type of business with barges being positioned.

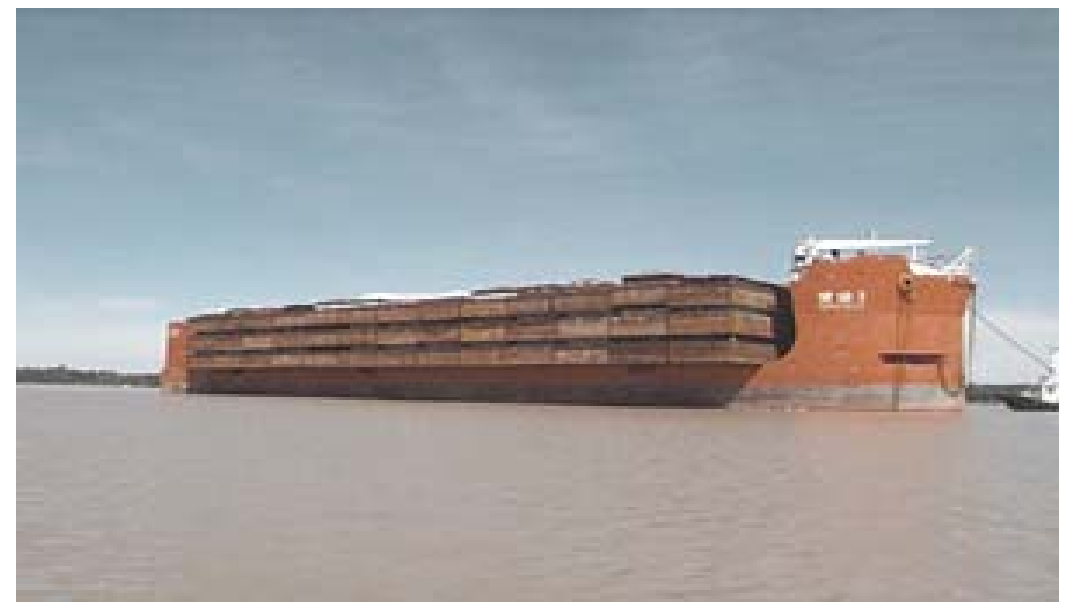

Figure 7-1: Barge carrier positioning barges in the River Plate.

The capital concentration phenomena brought about a deepening of the situation that had been taking place with respect to historic market informality during the soy export season. Unlike the 1980s and early 1990s, the market structure changed with a single player emerging to take the lead with respect to whether or not to commit with long term contracts to carry iron ore from Corumba (Brazil) to San Nicolás (Argentina).

Even though this barge operator engaged in one year contracts, it was normal practice to break down a convoy and split it to carry soy products at the expense of leaving the steel company without these badly needed supplies. While on the one hand the penalties for breach of contract were often substantially lower than the additional income soy freight rates generated; on the other hand the soy exports seasonality did not hinder the barging company from operating informally. This was because competition was not relevant given the large iron ore volumes required.

Therefore, the steel firm's critical stock was fed from Australia, USG, or northern Brazil, as a way to cope with the market informality and supply uncertainty that dominated the waterways in those days. Figure 7.2 illustrates barges being transported. 


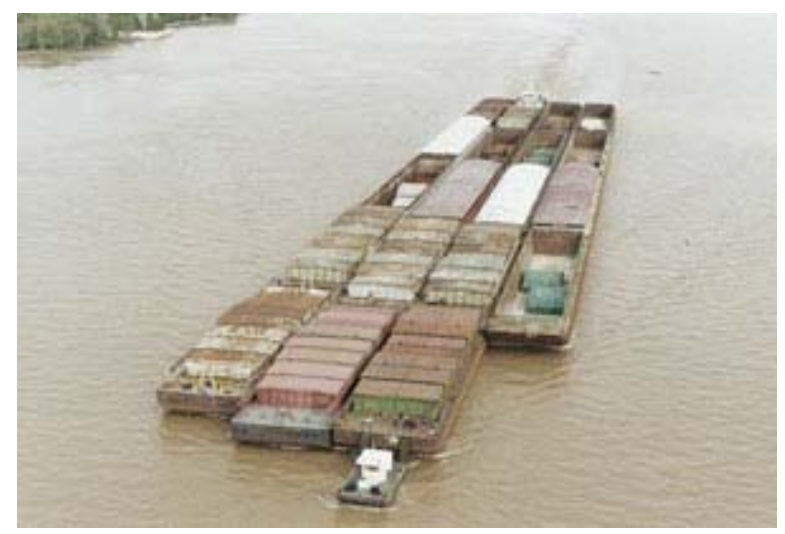

Figure 7.2 : Typical barge convoy represented by multi-purpose barges based on informality and poor performance.

It is useful to visualize some navigation distances, convoy formation structures, and draft limitations to allow the reader better understand the formidable operational constraints the waterways presented:

1. Total river navigation distance: $2,450 \mathrm{~km}$;

2. Convoy formation structure: 12 barges on $3 \times 4$ mode basis 200 meters in length, 45 meters in width; carrying capacity of up to 2500 tons each = total 30,000 tons;

3. Tugboat pushing power of 4,000 HP equipped with bow-thruster and having an optimal operational draft: 10 foot 6 inches;

4. Draft limitations: March through September 12 foot - September through December 9 to 8.5 feet - January to February 8 to 6 feet.

Further operational particulars:

- Critical passages ${ }^{82}$ marking trends in the river are found at various spots all along the waterways. However, there are 500 kilometers on the Alto Paraguay river (km. 0 to 500) that show certain particulars that are worth highlighting;

- $\mathrm{km} .485$ rocky bottom: barge convoys could not dredge the river bottom by just pushing forward and making use of the tugboat power, or becoming fractioned to facilitate individual barge (rather than barge convoy) passage.

\footnotetext{
${ }^{82}$ A critical passage is one that shows a lower draft than average or where a convoy needs to make special maneuvers to avoid a physical obstacle during it navigation.
} 
- km. 415 rocky and sandy bottom: barge convoys could not dredge the river bottom though could be fractioned and facilitate passing carefully right on top of the sandy bottom by leaving rocks on either side.

- $\mathrm{km} .184$ rocky and sandy bottom: idem km. 415, though facing strong currents that posed serious constraints to a safe maneuvering of both tugboat and barges.

The above clearly shows that every single voyage had to be carefully planned in advance and get the convoys loaded with as much cargo as possible, though always considering what data nature provides to the experienced eye about hazards. Often highly skilled operators could 'read' warning signals displayed at a given moment with respect to a spot lying some $800 \mathrm{~km}$ away. Therefore, data readings at a given moment often led to a disastrous round trip operation for all those who participated in the sailing adventure because of inherent uncertainty of weather, river and other relevant factors that need to be expertly 'read'. Even if data readings were correctly interpreted and expertly put into practice by the barge operator, much was left to the operator's tacit knowledge on how to successfully navigate these turbulent waters for a safe arrival. This kind of operation suggests the importance of traditional knowledge that in view of its very particular characteristics is critically strategic.

\section{4 - DESCRIPTION OF STAKEHOLDERS}

The project comprised a highly customized design that was developed to suit a series of complex logistic constraints of a steel-manufacturing company belonging to a large transnational industrial conglomerate. Project participants were located in Argentina and Paraguay. Geography influenced the project logistical design, as it is an extremely complex process to transport steelmaking raw materials by river barge over 2,400 kilometers across national borders along one of the world's largest river systems. River barge transport remains the most financially viable mode of transporting such materials. My reflection on this experience and feedback provided as evidence from participants interviewed both named in various tables and many others in less formal exchanges confirms that an important facet is that case study participants shared a past history of business interaction and collaborated through the Atlantic Corridor Project e- 
collaboration $^{83}$ network (Arroyo and Walker, 2004). This collaboration cemented trust and the necessary relationship commitment and goodwill that provided the underpinning for attempting to transform the way the collaborating firms participated in a value/supply chain. This innovative approach represents an interesting example of a project (that could be seen as part of a program or portfolio of projects) that helps explain how a business transformation was delivered through a series of projects in a portfolio of change initiatives.

The specific project focused upon in this study comprised the construction and operation of fully dedicated barge convoys to carry iron ore supplies from Brazil to Argentina commencing in 2003 and phased over six-years. The first phase comprised development and acceptance of an innovative logistics design solution and its realisation through the construction of new barges and leasing of existing barges. The project had in-built replication possibilities to roll out a series of similar projects. The following proposal as illustrated in Table 7.2 was accepted to describe the broad scope of the six-year project (with the prospect of a larger follow-on project):

Table 7.2: Project Proposal Scope

\begin{tabular}{|c|c|}
\hline $\begin{array}{l}\text { One fully-dedicated barge convoy twelve } \\
\text { months a year }\end{array}$ & $\begin{array}{l}\text { Building } 12 \text { barges of } 2500 \text { tons each }= \\
30.000 \text { tons capacity }\end{array}$ \\
\hline $\begin{array}{l}\text { One suitable tugboat of "pusher” type basis } \\
4500 \text { horse power }\end{array}$ & $\begin{array}{l}\text { Port Loading at: Ladario, Corumba, Sobramil } \\
\text { / Brazil }\end{array}$ \\
\hline $\begin{array}{l}\text { Speed } 5 \text { to } 6 \text { knots up-river and } 8 \text { to } 9 \text { knots } \\
\text { down-river }\end{array}$ & Port Discharge at: San Nicolás / Argentina \\
\hline $\begin{array}{l}12 \text { days sailing up-river; } 11 \text { days sailing } \\
\text { down-river; } 2 \text { days loading/unloading; } 1 \text { to } \\
2 \text { days open }\end{array}$ & $\begin{array}{l}\text { Gross operational time: } 25 \text { to } 27 \text { days; } 1 \text { trip } \\
\text { every month. }\end{array}$ \\
\hline $\begin{array}{l}\text { Total annual volume } 250,000 \text { tons }+ \text { or }- \\
10 \% \text { basis } 6 \text { years contract }\end{array}$ & 24 hours loading / 24 hours unloading \\
\hline $\begin{array}{l}\text { Barge dimensions basis } 65 \text { metres length by } \\
15 \text { metres width }\end{array}$ & $\begin{array}{l}\text { Building of new barges basis USD490,000 } \\
\text { each }\end{array}$ \\
\hline
\end{tabular}

The first barge unit required 90 days to be built with a maximum of 30 days for every subsequent built barge. A group of 12 leased barges was sourced, surveyed, approved and chartered from one

\footnotetext{
${ }^{83}$ Participants from remote and central regions across the MERCOSUR agreed to meet on-line twice monthly in a virtual meeting place with the aim of finding ways to solve logistical problems within a 24-hour period.
} 
of the smaller barge operators. Figure 7.2 illustrates a typical barge convoy. A reliable river operator was introduced into the project team and persuaded to operate the convoys under strict efficiency standards throughout the six-year contract duration. Three potential shipyards were approached in Argentina, Brazil, Paraguay and invited to bid for the barge-building contract using steel sheets for the barges to be supplied by the steel-manufacturing company from one of its plants in Brazil.

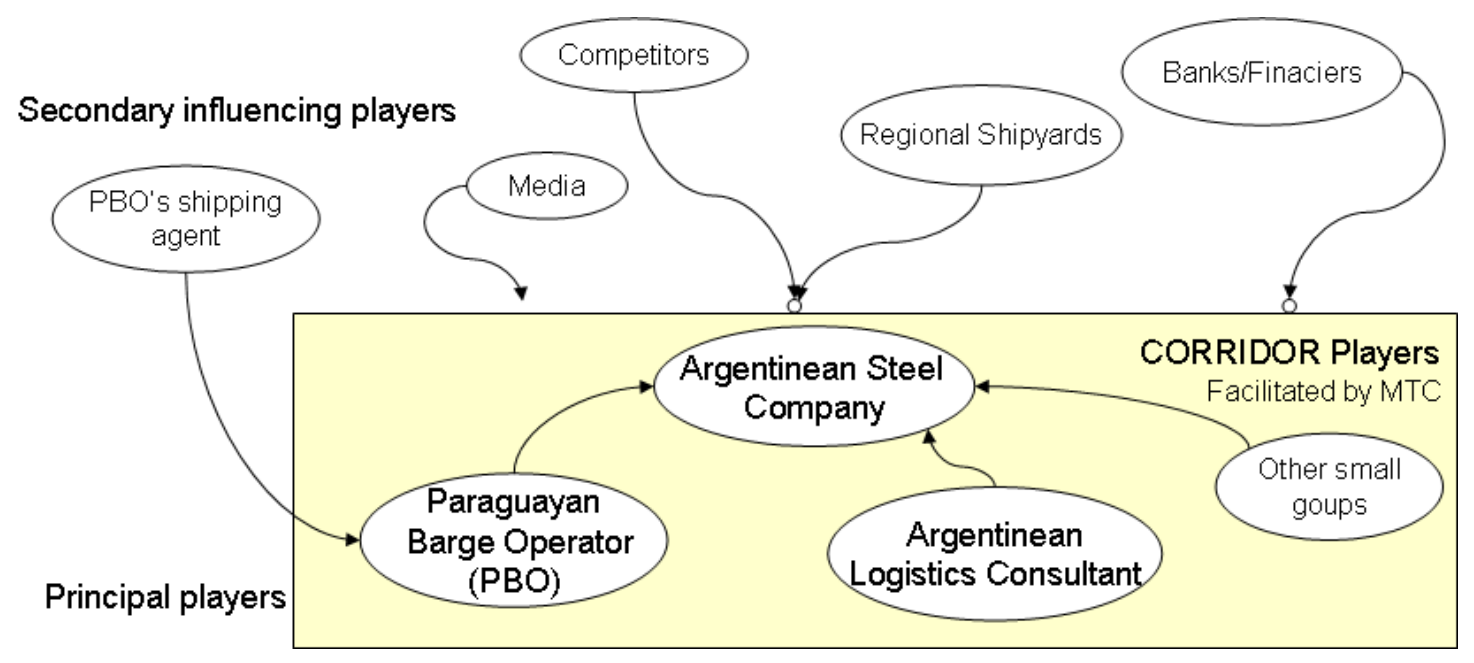

Figure 7-3: Joint Venture Showing Critical Primary Players and Instrumental Secondary Players

Figure 7.3 illustrates the formation of the JV that was facilitated by the MERCOSUR Trade Centre (MCT). It principally involved three principal players who formed the JV with several other instrumental secondary players that influenced the final JV design.

Although this project and therefore this case study addresses a number of players which made possible the implementation of the project itself, only those who played a key or critical role will be analyzed from a Corridor contribution perspective. Those having fulfilled a secondary role will only be mentioned to let the reader identify both the number of participants embodied in the project, as well as the different dimensions that made up the project, leading to a rather complex decision-making scenario.

Critical and instrumental players are described as follows:

a) Critical players or primary stakeholders: 
o The steel manufacturing company is based in Buenos Aires with 4,000 employees and having an annual turnover well in excess of USD 1 billion. This firm belongs to a much larger industrial conglomerate with annual sales for USD 3.2 billions and 16,000 employees worldwide.

o The logistic consulting company is a Buenos Aires-based firm specializing in the formulation and implementation of relatively high complexity logistic designs. It has offices throughout Latin America to provide industrial conglomerates with assistance in their logistic campaigns in landscapes characterized by difficult physical access. It specialized in oil, gas, power, and mining, infrastructure for customers represented by large transnational corporations with projects undertaken throughout the region. This company can be said to possess a great volume of tacit knowledge which provides them with a competitive advantage position as described by Porter (1985) of low inimitability along with an ever-increasing market focus positioning.

o The barge operating company is based in Asunción, Paraguay. This is one of the various river transportation firms that went through a reengineering process during the 1990s by disposing of most of their floating assets and shore facilities. However, this operator kept running a very efficient and rather small operation based on deep knowledge, solid planning, and sound strategy. It can be said this operator was probably the only left on the waterways skilful enough that to undertake a project of this nature as well as being daring enough to challenge larger and more resourceful global operators.

b) Instrumental players or indirect stakeholders:

o The barge operator's ship agent is based in Buenos Aires and could be considered the barge owner's right hand in Argentina. A barge owner himself during the 1990s and ex-partner of the Paraguayan barging company, this ship agent has become a shrew and intimate translator of the various meanings and codes that are often hidden behind people's facades, attitudes, phrases, and cultural behaviors. The ship agent acted mainly as a cultural bridge and managerial interpreter between the Argentine steel transnational and the Paraguayan barge operator, as both the current operational and regulatory framework on the waterways resulted being well known fields by the Paraguayan operator. This actor played more of a role as a cultural enhancer than as a technical advisor on operational topics one might assume were of tactical relevance. 
o Main competing shipyards in Argentina, Paraguay, and Brazil played their respective cards by actively lobbying both critical and instrumental players alike. Technical skills, social contacts, governmental lobbing, and community impact were some of the variables cleverly utilized by the shipbuilders and the client, suggesting a fluid interaction of conversation and knowledge exchange at the corridor roundtable forums.

o Main competitors were wary of capital concentration providing too much dominance over the waterways market. Competitors' retaliation to impede the accomplishment of the project was not less significant than their seemingly short-sighted strategy of excessively relying on their prevailing market dominance. The Corridor also provided a dynamic scenario for interactions to occur between all competitors who were members.

o Banks were purely instrumental in approving or rejecting the project from a financial standpoint, although their understanding of the waterways business may be regarded as important.

o The media also became instrumental in supporting through editorials and articles lobbying in the press and TV the location where barge-building was going to take place as well the facilities wherefrom the steel was going to be supplied for the shipyard to build the barges.

\section{5 - INSIGHTS ON THE PROJECT LOGISTIC DESIGN}

The project's logistic design was formulated by the Buenos Aires-based consulting company on the basis of carefully assessing the existing contextual conditions and the steel manufacturing firm's strategic position at that given moment. Such an assessment entailed a deep review and study of the following aspects:

1. Australia’s increasing commitment to supply China's growing demand for iron ore;

2. USG suppliers' growing preference to meet China's increasing demand for iron ore;

3. Northeastern Brazilian suppliers’ preference to supply both China and India's expanding markets for iron ore;

4. Inauguration of a new plant in the River Plate to cope with a steady increase of demand for steel-made end products from all over the world - Asia-Pacific region inclusive;

5. Existing relative indifference on the part of the steel producing firm with respect to becoming more actively involved in controlling their own waterways-based supply-chain; 
6. High levels of capital concentration of river transportation companies operating on the waterways linking the Brazilian suppliers with the Argentine buyer, leading to both price increases and service informality;

7. High levels of on-going variability and future uncertainty scenario for oil prices impacting negatively on ocean-going vessels' time charter market;

8. Steady increases in demand volumes for soy products arising out of China's formidable growth leading to a river transportation imbalance and a general rate increase in barging services; and

9. Slow reaction and limited innovation capacity on the part of the Argentine steel-producing company despite its global structure and well reputed international management.

In view that the waterways market was to a great extent dominated by a major player and that no independent barge operator was at that time in condition to successfully compete on a sustainable basis, the logistic design illustrated in Table 7.2 was formulated and submitted to the steel company for their evaluation. It was intended to both bring an achievable solution to the multinational and to make their executives visualize the opportunities lying in the long run in terms of market dominance, ship-building, and supply the derivable outlined in Table 7.2.

The building project schedule was expected to be 90 days for the first unit and a maximum of 30 days thereafter for every barge, bringing the total construction time up to a maximum of 12 months. In the interim period a group of 12 barges was located, surveyed, approved, and chartered from one of the smaller river companies that were still making business on the waterways. Figure 7.3 illustrates a typical example of one of these second hand barges.

The steel-manufacturing firm signed a contract for six years, this being a requisite for the financial institution to even start talks and carry on with the process. A reliable river operator was brought into the deal and convinced to operate the convoys under strict efficiency standards throughout the duration of the contract and despite the well-known operational constraints of the fluvial system itself. Three potential shipyards were approached in the three countries (Argentina ; Brazil ; Paraguay) and were invited to bid for the building contract. The steel sheets for building 
the barges was agreed to be supplied by the steel-manufacturing company from one of its plants in Brazil in view of it having the most adequate sheet cuttings and lowest cost.

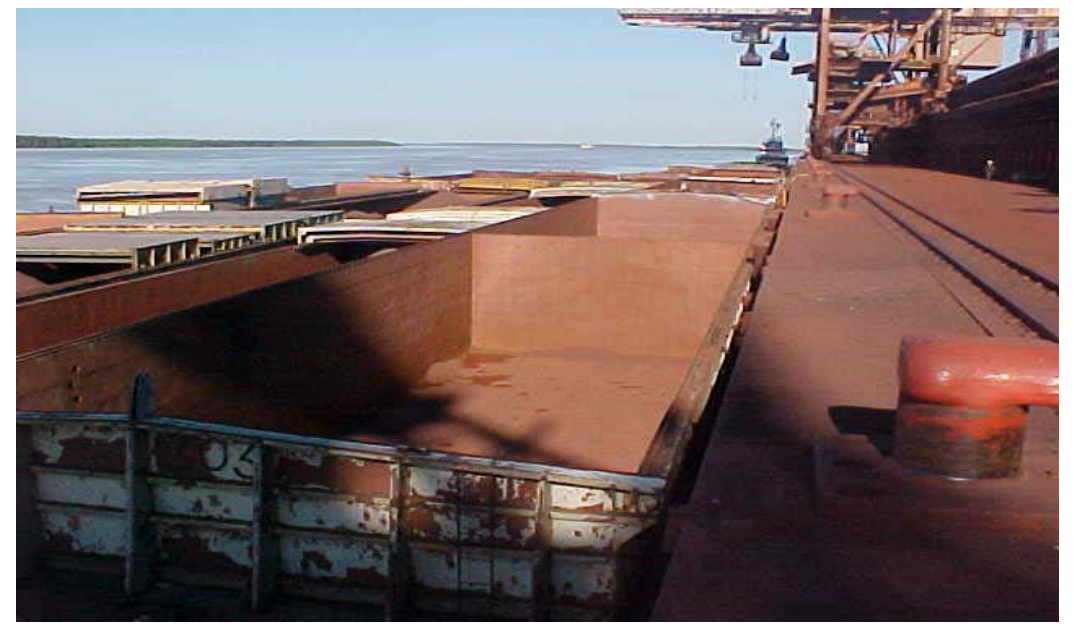

Figure 7-4 : Second Hand Barges Getting Ready to Start Loading Operations Alongside Berth in Corumba, Brazil, During the Interim Period .

The moment this information appeared in the media, things started to change and the project ran into serious danger of running aground and therefore obstructing the realization of a second larger contract upon which negotiations were already being held. It is interesting, and aids making sense of the context of the emergence of this project, to break down and later analyze from a leadership standpoint - item by item - how the sequence of events was finally influencing the players' decision-making and shaped their final agreement - it reflects a somewhat typical project multi-headed stakeholder quandary that needs to be resolved. It also illuminates the reality of those involved in PM that reflects the lived reality of being involved in PM activities in all its uncertainty, planned responses as well as coping mechanisms to achieve results.

A Memorandum of Understanding (MOU) on a six-year contract was made public on the media by March 2004. Details of the events that followed are summarized as follows:

1. The dominant and largest river company lodged a formal claim on very old pending issues against the smaller river operator and proceeded to seize some of the barges that had been chartered to the steel-making firm for the transition period of 12 months - until the new- 
buildings were launched and ready to operate. Thus, the first shipment of 25 to 30,000 tons of iron ore resulted in a failure.

2. The steel-making company's shipping manager threatens the large river operator with no longer chartering their ocean-going vessels from Northeast Brazil - as they also provided shipping services from overseas - unless an amicable solution to this issue was found.

3. No threat was made however, with respect to the on-going barging operations of this dominant player as despite being informal and therefore inefficient, the steel-producing firm needed to maintain raw material supplies in any way it could to avoid having to make use of critical stocks at San Nicolas port terminal at their plant in Argentina.

4. The Paraguayan operators reinforced their distrust of the smaller river operator by sustaining belief that one never knows what legal or financial problem lies behind this firm's floating equipment assets: maritime liens; embargoes; outstanding moneys; material deficiencies; unsettled casualties, etc. In the meantime, the Asunción-based company had positioned a tugboat in San Nicolas while waiting to proceed with the voyage, thus incurring in heavy running costs.

5. The Paraguayan operator offers to personally talk to the smaller river operator in order to convince him to pay out the pending moneys, lift the embargo, and carry on with the river adventure while getting capitalized with the still pending 12 months charter. Instead, the smaller operator proposed that the Paraguay-based company advance the said pending monies in order to immediately free the barges and to jointly exploit other extra-contract barges that were also trapped by the embargo to carry other party's cargo with their tugboat. After some robust negotiations, the deal was accepted and the first convoy departs from San Nicolas towards Asunción - though a high degree of distrust is retained by the Paraguayan operator.

6. While performing the $3^{\text {rd }}$ day of navigation a new embargo emerged and resulted in 6 out of 12 barges being seized and detained at an intermediate river port. This time the plaintiff was the Argentine Government that claimed reimbursement of some loans that were destined to construction but that were in fact utilized on running their daily operations. Lawyers had to be appointed and guarantees were submitted to let the convoy proceed. However, the efficiency degree with which the whole transition process of the river operation had been planned was already void, and deserved an urgent reengineering if economies of scale were to work on their side instead of becoming a over burdensome. 
7. The dominant river operator let the market know they had allocated a huge building order for 40 barges in each of the potential shipyards the steel-making firm was negotiating with. The most likely shipyard - located in Paraguay - acknowledged not only having read the news but also being in advanced negotiations to build 40 barges as informed by the media. Whether this was a distractive operation or a strategic move to keep control of the waterways market was by then unknown to the players.

8. The financial party started to delay the final signature for releasing the funds in view of the recent events which brought about uncertainty and made public their concern on whether or not the appointed shipyard would have enough building facilities to carry out the job on time and within budget, these being two variables of paramount importance in a project characterized by efficient operations based on low margins.

9. The steel-producing company instructed their lawyers to speed up the proceedings of some old claims for barge sinking and total loss they went through on several occasions against the dominant river player which they found to be responsible for. Clearly, this acted as retaliation.

10. The media gave ample nationalistic coverage to the fact that an Argentine company was placing a construction order not in Argentina but in Paraguay, and to supply steel sheets from their Brazilian subsidiary and not from any of their various domestic facilities.

11. The Paraguayan operator finally lost the support of their financial partner and had to call for the services of another bank, which demanded less attractive conditions causing margins to lower even further - though still feasible.

12. The Paraguayan shipyard - though the best qualified - was eliminated by political motives and thus two Argentine shipyards became short-listed. One turned out to be very knowledgeable and counting on respectable facilities and availability to start works immediately while guaranteeing a strict construction schedule compliance; the other one was once a large yard specialized in building railway wagons and whose CEO happened to be an old school mate of the steel-making company's owner and chairman. In spite of not counting on the right facilities and giving no certainties with respect to construction timing or delivery dates, they were invited to participate in the bidding process. 
13. The new bank, despite knowing little about naval construction engineering, became the first party to oppose the appointment of this new shipyard by alleging their lack of expertise and commitment.

14. The Paraguayan operator confidentially let the others know that should the bank pay a visit to this shipyard's job-site the deal was going to be over again, as it was clear that their facilities did not match the basic needs of a safe construction process.

15. The steel-making firm's managers had no other option than considering this shipyard's proposal in view it was somehow favored from the very top of the organization.

16. Such a proposal delayed over and over and made the Paraguayan operator give a final notice to the steel-making firm by alleging they could no longer wait for a final decision if an operational scheme based on efficiency and productivity was to be accomplished.

17. Finally, the inexperienced shipyard delayed too much in matching the necessary technical requirements and their offer was dismissed, despite of having assured to be by then, the most competitive. Despite the fact that they were previously disqualified, the bid was won by the Paraguay-based shipyard while the steel sheets supplies were agreed to be sent off from an Argentina-based facility as a way to cope with the media and pacify potential critics by referring to the fact that the whole project remained as a MERCOSUR deal, with no extraregional participants. However, at the very last minute the board of the steel-producing company decided to proceed and place an additional construction order with the most qualified Argentine shipyard and therefore commit to the fully dedicated operations philosophy to be applied on a $2^{\text {nd }}$ convoy.

18. By-products of these first two contracts resulted in a potential third identical contract being awarded to the same Paraguayan barge operator through the consulting company with shipyard works completion monitoring and the carriage of steel sheets being awarded to the consulting firm.

19. Related business possibilities emerged for the consulting firm with respect to developing a fully-dedicated convoy for a large soy exporter further to a new river traffic for the same steel-manufacturing firm.

These simplified vignettes of the complex and energetic interactions between stakeholders provide a glimpse of the complexity of the situation. Delivering a business transformation of this 
nature is fraught with uncertainties, should-be obvious (but often ignored) risks and politics of stakeholder management that can only be summarized and alluded to. The lived experience is far too complex to describe in terms acceptable to academic studies because so many of the issues trigger emotional and therefore highly personal, contextual and tacit responses and reactions. The point of highlighting the above is to illustrate the complexity, richness and tentative nature of variables involved. Such studies are necessarily filtered by personal observations and experiences of those contributing evidence. However, despite these limitations, the lived experience advances our knowledge of repertoires of behavior and responses to such situations and so it is this value that constitutes the value of these business transformation project management studies.

\section{6 - THE CORRIDOR AS A FACILITATOR}

Two driving forces for this joint venture become clear from this case study; one is the global dimension of the primary stakeholder (steel manufacturing company) and the other one the complexity of the logistic operation in itself that made traditional knowledge of strategic relevance. One might assume that the steel company, holding a business presence in so many countries around the world and having access to countless data bases and logistic experiences to feed from and benchmark with, would not regard the Corridor as a potentially valuable development tool.

However, not only did the primary stakeholders interact during the roundtable forums and exchanged knowledge until eventually giving birth to a knowledge-based logistic design, but also the secondary stakeholders took part in the process and became interested to form part of what was about to take place. Even though a number of functional aspects such as financial engineering, insurance, port operations and barging services were often contracted by the steel company on diverse geographical settings, the knowledge specificity and viscosity of the logistic design along with its very unique geographic and operational particulars embodied by the waterways, seem to have found a fertile ground for these various actors to get together and become interested in one another's needs, potential, and proposals.

Carlos Candia, Project Manager of the Buenos Aires-based logistic consultancy company, attended the roundtable forums long before this project became a reality and therefore had the 
opportunity to keep face-to-face contacts and dialogue with various members who later brokered him to some of the senior executives that played decisive roles in this project. In one interview with me he said (translated from Spanish) "I always attended the roundtable forums as I considered them a unique opportunity toward exercising a realistic knowledge sharing and transfer, as well as a suitable arena for networking in search of future business opportunities. However, an aspect I immediately realized and caught my full attention on the river supply problems the steel company was going through, was its high complexity, a great number of variables to consider, the risks involved, and perhaps its very uniqueness given its inimitability or difficulty to replicate anywhere in the world".

When asked on the way the steel company's executives reacted to the globalization impact he replied; "It was amazing to see the contrast between the apparent available resources of a globally established and well reputed international company (where one might assume there will always be an alternative strategy to follow), and the lack of vision or anticipation of their regional executives to come up with a solid plan to break their logistic dependence on a single barge operator".

According to Carlos Candia the Corridor proved fruitful for this large company in that their executives had the opportunity to witness the truly dynamic character the Corridor held during the peak of the globalization impact over the region, as well as the speed at which knowledge sharing and transfer took place in its different forms. In his opinion, the roundtable forums for the steel company seemed to have taken the leadership role of that of their company's most experienced managers, who as well as holding a recognized leadership by peers and subordinates, also find themselves in a pyramidal structure where levels and ranks are respected and followed. "The multinational's managers looked so structured and accustomed to be told what to do and how to carry on as if they did not have any initiative on their own, which was a weird supply chain situation as it was the Corridor the one that precisely adopted a leadership role and acted as a truly motivating tool for them and others".

MTC’s President Roberto Cristaldi also interacted with several of their executives as well as participated in numerous roundtables while integrating various CoPs. He observed that these 
executives had taken the typical attitude of those more interested in assessing others' ideas and concepts rather than developing their own formulas. "I have seen this attitude many times amongst executives of the larger companies who were convinced their firms' potential volumes or purchasing power was large enough to adopt a come-to-see-me and shall assess your proposal attitude - instead of timely reacting and putting into practice their own plans to weather the storm”.

Roberto Cristaldi has a coincident opinion with Carlos Candia in that the Corridor proved an efficient arena to open up these executives’ minds towards becoming more receptive, challenging, realistic, and perhaps inspirational to face an uncertain scenario. He also highlighted the very specific role traditional knowledge played not only with respect to the barging operation, but also from a financial standpoint. "I believe that the uniqueness of the waterways operations is very clear and out of discussion. However, there seems to have been also a financial scheme that was not readily available to be developed by just any bank or financial institution as it demanded to truly comprehend the operational risks involved and the very particularities of a 2,400 $\mathrm{km}$ of fluvial transportation. In this regard I believe the Corridor played a fundamental networking role for the members to profit from as well as it meant a tremendous innovation incubator in the sense that companies attended not just in search of new businesses, but that were just compelled to touch base with the right party to face the new scenario".

Mariano Gramajo was the operations representative of the Paraguayan river operator in Buenos Aires. His main observations are related to the framework within which the project developed as well as the various stages that were needed up to accomplishing the final logistic design. He argues that it is difficult for him to imagine this project having moved forward in a more traditional setting such as business or informational meetings held at the ship-owners association or chamber of commerce or some sort of internal follow-up management gatherings, or under a typical management by objectives scheme arising out of a particular company need.

He said: "Firstly, there is a rapidly changing business environment that is undoubtedly impacting both on the region and upon all the players. Secondly, the business context uncertainty only rivals the almost countless variables a 2,400 km all-year-round river operation embodies. 
Thirdly, the magnitude of the traditional knowledge along with its complexity is so large that an independent or outer development becomes simply unthinkable”. To this end, Mariano Gramajo believes the roundtable forums proved to be critical for a subsequent assembling of the different parts and pieces of a very complex logistic puzzle.

Carlos Arizaga was the steel company’s Procurement Managing Director and head of the waterways project within his organization. He eventually submitted the project for the approval of the board (once the consultancy firm had rounded off the logistic design). He headed a division of more than 20 engineers in charged of the many logistic segments needed to keep various plants running across Argentina. He admitted that his team was more focused on setting new key performance indicators and improving or securing their supplies from the various origins, rather than on innovating on new supply channels or creating the conditions for new logistic suppliers to emerge and grow along them. When asked about this particular logistic design he was categorical in his response; "The parties to talk to were so many, their interests and points of view so diverse, and the traditional knowledge so deep and attached to every single player, that I find an alternative setting to develop a project this complex simply unrealistic”.

I requested the river operator representative's opinion on the above comments. He stated that had this individual and his team taken a more modest attitude or deployed a better receptivity with respect to those holding the specific operational knowledge, the project would not have been so time-consuming. "With this I confirm my own suspicions in that representing a large multinational may give oneself negotiating power and a high self-esteem I suppose. This person along with his team took a long time to evolve and understand that here the key to the project was accepting others' views and experience coming from so many years of working on the waterways, and not that much engineering performance ratios and indicators that proved successful somewhere else. I am convinced this was always about who held knowledge and not so much about relative power or global dimension”.

I had the opportunity to participate in the logistic design developed by the consultancy company (which brought together the primary and secondary stakeholders and shaped the logistic design in itself) while at the same time attending the roundtable forums as a Corridor member. In my 
opinion not only the Corridor resulted key to let people interact and participate in different CoPs, but also was a suitable setting to replicate proposals and countless situations by third parties who were not part of the project but that possessed a specific piece of knowledge applicable to different stages of the project. Their own opinions and views (based on their past experiences) on a number of different functional aspects (e.g. loading speed at Brazilian river terminals / controlling procedures by the national coastguards / port congestion impact on freight rates / casualty probability / seasonality impact on round-trip performance) helped immensely to dissipate the doubts or fears of the steel company's management before a scenario that they could not dominate, anticipate, or even entirely assimilate.

Even though most of time this whole exercise was not an easy journey, such a process provided the various players with the possibility to double check with those who were not parties to the project on certain operational issues, which otherwise would have possibly meant the unfeasibility of the project owing to potential doubts with respect to operational or financial aspects, hidden agendas, or simply lack of trust. A complete breakdown and full exposure of the critical variables along with the input coming from both the stakeholders and collaborators helped the project to avoid running the risk of becoming inconclusive.

Ruben Conde, shipping financial expert, was in charge of designing the financial engineering that made possible the different pieces coming together. Even though he did not regularly attend the roundtable forums, he had the opportunity to witness its functioning and corroborate that his comments on specific financial issues that were brought up from an operational perspective, helped to consolidate the players' visions that otherwise would have probably led to nowhere. He said: "I found the Corridor's dynamics very interesting and challenging. But perhaps most importantly was the participation of those who were not central to the project but that based on their experience contributed to dissipate doubts and increase transparency and trust amongst those who were critical to the project".

In my quality of direct participant and observer I agree with those who commented on the uniqueness of this project as well as the great number of variables that had to be considered on a 12-month basis operation. In this regard I would like to add that a river operation holds a tight 
connection with its geographical environment which is very unlikely to extrapolate in view of both the existing topography and the traditional knowledge that often comes attached to it.

Even though I had to operate convoys on both the Amazon basin in Brazil and the Orinoco basin in Venezuela, I find them both unique and therefore different when compared to each other or with the Parana-Paraguay waterways. One can always find certain analogies though they often turn out to be the exception and not the rule. In those locations I also found traditional knowledge to be absolutely critical while both the operational variables (tug boats; barges; terminals; cranes; berths; buoys; people; culture; etc) and the geographical particulars (depths; tides; currents; sedimentation; seasonality; rains; fog; temperature; etc) which tended to be also unique.

The next section will address the case study conclusions based on the axis of both the role played by traditional knowledge and the Corridor's dynamics in allowing a project of this nature to be formulated and put into practice.

\section{7 - CHAPTER SUMMARY}

There are three aspects that clearly stand out in this case study that perhaps did not play such a dominant role in the previous examples. One is represented by the fact that once this project was designed, formulated, and eventually put into practice, the worst of the globalization impact on the region had already been experienced or absorbed. Therefore, the driving motive of this project does not find its sources on the first globalization wave over the region, but on a sort of globalization impact byproduct that brought about a specific supply-chain problem to a major regional player.

The traumatic moments that hit each and every national and regional logistic player and compelled them to reengineer their business or give up, were over by the time the steel company had engaged in the roundtable forums. It can therefore be argued that the Corridor as a system, as well as its varied array of members, were pretty much conversant with its modus operandi and had witnessed or taken part of the many alliances and JVs that effectively took place. Consequently the Corridor counted on a certain level of maturity acquired during the 1990s by the time the steel company joined the system in the late 1990s. 
The second element is given by the high level of traditional knowledge a project like this one needed to develop and become a reality. Had not the Corridor counted on those few critical regional players having a thorough understanding or deep knowledge of their business context, it is extremely unlikely this project would have progressed the way it did - regardless of the contextual or global circumstances. Bringing together potential independent actors with a common interest or rationale to collaborate, exchange views and impressions, and transfer knowledge to produce a remarkable business transformation on the primary stakeholders, would have been unthinkable without identifying the existence of a solid traditional knowledge platform to grow from.

This project knowledge specificity and viscosity and its apparent dependence on traditional knowledge, also suggests that a certain degree of maturation amongst the Corridor members as well as the roundtable forums' functioning, was needed to overcome the many managerial and operational barriers along the process. One may assume this project to have failed had it been undertaken by the early 1990s when the system was just initiating and little experience was available in terms of interactions, networking, knowledge sharing and transfer, cooperation, collaborations, etc.

The way stakeholders (direct and indirect) and outsiders collaborated toward bringing data and information on their own functional experiences shows that further to traditional knowledge, face-to-face interaction along with a given accomplished maturity degree of the system in itself, have also held a triggering effect towards the project completion. It seems clear that trust, commitment, leadership, motivation, collaboration, and innovation have all played their part within a common setting.

Even though it was not the globalization impact of the early1990s (the one event that triggered the reaction of the steel manufacturing firm), a comparable outer symptom was aroused by China and India's economic expansion — which had a similar effect on the main stakeholder when compared to the logistic companies at the outset of the Corridor. A critical element for the steel manufacturing firm's decision to join the Corridor was their being unable to rely upon in-house 
resources (or perhaps lacking of vision, initiative, or leadership) so they needed to search for knowledge, innovation, and possibly leadership to deal with this deep rooted knowledge-based project.

The third aspect that stands out in retrospective reflection of my role as participant and catalyst for change, was that rather than there being a highly formulated strategic plan painstakingly designed at the outset (that according to Mitzberg, Ahlstrand and Lampet (1998) can be classified as fitting the planning or positioning school of strategy where a more stable and predictable environment prevails) the project followed an emergent and learning school of strategic thought — with elements of the entrepreneurial, cognitive, cultural, power, and environmental schools. Indeed to follow their typology of strategic decision making that this case study best exhibits, the way the project unfolded is more in line with the configuration school which is best suited to dealing with turbulence, uncertainly, a need to be agile and responsive. The transformation in PM approaches that this case study illuminates is how different the approach adopted to deal with the many primary and secondary players and the economic environmental realities that this approach exhibits when compared to how this industry segment would traditionally be approached. The Corridor and its facilitation not only influenced critical elements of knowledge exchange and co-generation, and its brokering activities as a business opportunity incubator but it also appears to have facilitated a more pragmatic and configurationally strategic approach that all parties adopted.

In my privileged role of direct observer I can affirm that when the impact of the Asia-Pacific economies had taken place, the Corridor members had already to a great extent reengineered their businesses to cope with the new business context that gradually impacted the region during the 1990s. This entailed a great deal of business transformation along with vision, knowledge sharing and transfer, and leadership on the part of the members with respect to both their own internal or regional reengineering, and their alliances and partnerships with foreign-based companies. Such a degree of evolution has apparently helped the steel company to soon realize they had to open up and push its executives to interact within the roundtable forums in search of knowledge to transform their business limitations in strategic opportunities. 
To this end and given the project complexity, the maturity accomplished by the Corridor by the early 2000s seems to have helped the steel company transform a threatening supply-chain context into a more predictable operational context leading possibly to, a dominant market position in the waterways. Further to securing their logistic supply sources, the Corridor proved efficient in finding ways to enlarge economies of scale to gain competitive advantage.

The next chapter will develop a cross study analysis of all the case studies presented aiming at discussing the findings that seem to be common to all, highlighting the concepts where the Corridor seems to have been successful, and making reference to those aspects the Corridor needs further development or a different strategy to improve its overall performance. 


\section{CROSS-CASE STUDY ANALYSIS}

Chapter 6 and 7 presents data, findings and insights from a variety of triangulated sources. These findings were then considered from reflection and analysis, both from a grounded data up theory and from a top down literature perspective, led to overarching themes to emerge. These help explain the role of the Corridor in this context and to address the research questions.

\section{1 - INTRODUCTION}

This chapter starts by addressing the end of a sort of linear dynamic growth model that the various logistic segments experienced right before the globalization reached the region. It describes Porter's (1985) five competitive forces as a suitable vehicle for companies to expand and invest as well as his three main strategies to gain competitive advantage. A cross-case study analysis is made with respect to the way Porter's (1985) three main strategies helped companies to face the upcoming business context along with the Corridor's contribution along the process. The chapter further continues with a cross-case study analysis of Porter's (2001) value chain model and the actual contribution of the Corridor to the different roles played by both the primary and support activities.

Another cross-case study follows and embodies the different aspects that both transactional and transformational leadership types influenced each project as well as the way the Corridor turned out to be critical to help bringing up leadership to the benefit of the stakeholders. A final crosscase study on knowledge management highlights the importance and influence of Nonaka's (1991) SECI cycle as well as relates Dixon's (2000) knowledge transfer types to each case study aiming to better comprehend the constructive role both the Corridor and the roundtable forums played at that time. The chapter ends with the conclusions where both the achievements and failures of the system are highlighted in order to open up a new avenue of discussion in the following chapter.

\section{2 - CROSS-CASE STUDY COMPETITIVE FORCES ANALYSIS}

The moment the globalization process hit the region and together with it the market opened up to new options, the growth dynamics changed and the need to adopt some sort of business 
transformation strategy became apparent on the part of the stakeholders - either primary or secondary.

Case Study 1 (shipping JV project) explained the drastic transformation a number of shipping companies had to go through to remain competitive. Case Study 2 (land-bridge project) involved two companies that had to face a significant organizational transformation to achieve a level of innovation capable of facing a new non-linear dynamic business scenario. Case Study 3 (regional railways project) details the ways for a solid national company to reengineer and become first regional and later international to plan how to keep control and respond to demand and achieve the necessary economies of scales to remain competitive. Case Study 4 (port terminal project) was perhaps the most relevant example of the way a linear dynamics business environment can drastically turn into a non-linear one that leads to uncertainty and ambiguity with respect to planning the future. Case Study 5 (river transportation project) clearly illustrates the way outer regional uncertainties can constructively trigger a profound organizational transformation even within a supposedly rigid and hierarchical multinational company.

It is in this respect that all five of these case studies easily identify with Courtney, Kirkland, and Viguerie's (1997) argument that in turbulent and rapidly changing circumstances, traditional strategic planning horizons are dangerously constricting and confining, leading to a true ambiguity situation (level 4 of their model). This situation provides no basis to forecast the future but rather, it requires drawing analogies, recognizing patterns, and putting into practice non-linear emergent and recursive models. So it becomes hard to imagine how long it would have taken for all these firms to effectively internalize, assess, discuss, and benchmark with others the various alternatives to better face a threatening scenario. Clearly a mechanism that provides a safe space or $b a$ to explore situations, scenarios and options is both necessary and desirable.

Figure 8.1 illustrates the struggle that was going on by the case study organizations as they tried to balance forces acting upon them with strategies and actions they could take and how they could shape the future by managing the delicate balancing act. 


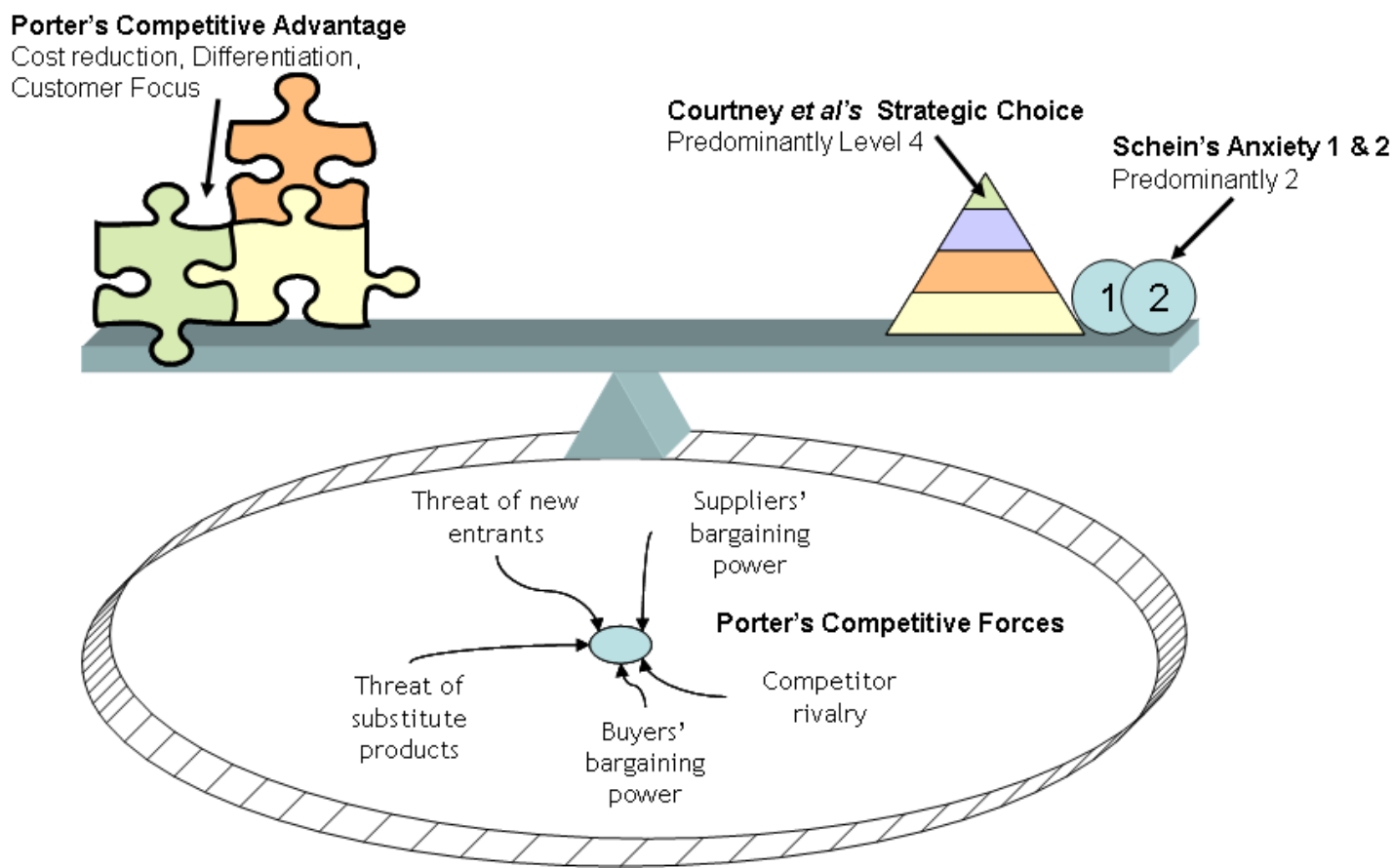

Figure 8-1: The Balancing Act

The Corridor at this point seems at least to have shortened networking times and provided a unique setting for companies to benchmark with one another, capture others' ideas or thoughts, imitate constructive attitudes, and erode potential rivalries to slowly shape the minds and spirits of the members to let cooperation, collaboration, knowledge, and leadership to emerge. Had not the Corridor project existed, one could well assume that the globalization impact would have hindered many firms from achieving larger economies of scale on time to remain or even gain a more competitive profile.

Even though the Corridor gathered a wide array of companies together in these transformation projects with a variety of visions and interests (also represented in the five case studies), it is interesting to highlight its potential role to bridge the existing gaps with the two types of anxieties as described by Schein (1993) in Chapter 3. Anxiety 1 holders (fear associated with a company's inability or unwillingness to learn something new in view of its difficulty degree) while anxiety 2 holders (fear, shame, or guilt for not learning something new). Both anxiety types seem to have had their differences eroded owing to the existing cross-business learning dynamics along with 
the concept of a growing uncertainty level that acted as a triggering effect for members to open up and show more receptive.

Porter (1998, pp8-11) argues that even though industry profitability in the short run depends on the balance between supply and demand, it is industry structure what determines long-term profits projections. In his opinion, it is industry structure that makes competitors add supply as well as reducing it every time an excess of capacity brings down the price levels to make a sector unprofitable. This is precisely the primary impact the region experienced from the globalization wave and what acted as triggering effect to drive the formation of the Corridor.

Porter's (1985) five competitive forces, which he describes as determining the ability for a firm to invest in a certain activity are precisely the fundamental concepts that supported the Corridor's essence which became commonplace in the five case studies. Entry of New Competitors and Threat of Substitutes are two concepts that are embodied in the case studies when facing the arrival of much larger-scaled and therefore lower cost-based global operators - with the exception of the river transportation project where the triggering concept was perhaps more identifiable with the already existing global market’s interdependence that suddenly and unexpectedly led this firm to undertake an aggressive business transformation.

The Bargaining Power of Buyers and Suppliers as well as the Rivalry Amongst Existing Competitors also are variables that Porter defines as critical considerations to companies' expansion and investment decisions. Coincidentally, the strength of these competitive forces reflect the fears and concerns of those firms that gradually joined the Corridor in search of alternative courses of action in a business environment that they could no longer control or even anticipate. These forces find similar expressions in all of the case studies with the exception of the last one, in terms of companies struggling to joint venture, rationalize, and reengineer to attain larger economies of scale while retaining their market positioning. Case Study 5 responds more to a structural economic transformation brought by the globalization process as well as an implicit desire to exercise leadership, and not so much to Porter's (1985) competitive forces strengths. 
The Corridor's contribution at this point seems to have been to facilitate the conditions for companies to actively interact and share their corporate views and concerns by effectively brokering a rather ample array of interests. Clearly, knowledge sharing and transfer stand out as privileged components in all the case studies.

Porter (1985) emphasizes that there are three main strategies for companies in order to gain competitive advantage: cost leadership / differentiation / focus. These three elements are present in the five case studies though adopting different forms, the Corridor being the facilitator for these strategies to spread and produce outcomes.

1. Cost Leadership: This became a critical variable for the shipping companies to attain during the JV process in case study 1 . It would have been very unlikely for them to individually face the larger firms deploying global economies of scale if they had not joined resources to rationalize their fleets and services in pursuit of greater efficiencies and broader geographical reach. The land-bridge case study would have never been able to successfully compete with the existing and well established full-container liners in the pacific if it was not for the shipping company's ample geographical reach (Western and Eastern Europe / Middle East / North Africa) that provided the land-bridge scheme with the necessary cargo volume to lower its operational costs and gain competitive advantage. Enlarging economies of scale was also crucial for ALL in Case Study 3 to effectively compete with both regional and extra-regional ground and river transportation firms. Case Study 4 also needed to first achieve larger economies of scale to allow it to later lower its operational costs - though pretending to face a global contender located just 70 kilometers away. The barging Case Study 5 JV formation would not even be considered to become a JV prospective option for the steel manufacturing company unless lower, or at least comparable, unit costs would not have been attained to make the waterways operations and construction a viable project. The Corridor seems to have fulfilled the role of an impartial setting for members to explore alternative ways to face the globalization as well as a fertile ground for knowledge, leadership, and culture to bridge gaps and let business transformation to occur.

2. Differentiation: Even though the modality of JV and VSA were well known in the northern hemisphere by the time the globalization wave hit the region, none of the 
shipping companies described in Case Study 1 (as well as in general) was allied or operationally combined with other market players but that all remained independent and disconnected from world trends. In this regard Case Study 1 shows a remarkable degree of differentiation in view of the unique and pioneering structure of the JV which by the time it was initiated happened to be a leading case for other to follow. Case Study 2 turned out to be not only unique, as it combined various modes of transport by bringing together two continents, but that also it was regarded as a leading case by most of the regional and global operators who happened to first observe it and then to later put into operations their own versions of the model. Case studies 3 and 4 also show a high degree of differentiation in that both formulated and implemented strategies that were not often seen even in more developed markets: a railway company that became regionally operational and globally commercial, as well as a national port terminal operator that challenges a world class operator by taking advantage from its deep knowledge of the market and customers. Despite the apparent geographical disadvantages of the waterways in terms of supply distance and operational constraints, Case Study 5 is a truly tailor-made logistic design where a higher degree of differentiation becomes hard to imagine.

3. Focus: Porter (1985) argues that focus means producing products and services that fulfill the needs of small group of consumers. As such, the 5 case studies focus on very well determined and specific market segments far from what a retail market strategy might look like. Focus was perhaps the most sought after strategy amongst the Corridor members when attending the roundtable forums, as a way to reengineer their businesses and survive while going through a transformation.

It becomes apparent that the linear dynamic concept was no longer valid in terms of business continuity or contextual circumstances. Anxieties seem to have been at first ignored by most stakeholders while they did recognize the seriousness of the globalization process and its associated potential impact. Although Porter's competitive forces find some similarities and differences in the case studies presented, they all seem to play a decisive role in shaping the firms' strategies to face the future. To summarize by referring to Figure 8-1, a combination of Porters' (1985) competitive advantage theory was balanced by consideration of the turbulent and 
uncertain context (as described earlier from Courtney et al (1997) and any influence of Schein's (1993) anxiety and awareness of uncertainty theory) explain the dynamics of the situation facing these case study firms. It is in this respect that the Corridor seems to have facilitated their crosslearning and managerial networking for them to profit from. The next section will address Porter's value chain model in relation to the case studies.

\section{3- CROSS-CASE STUDIES VALUE CHAIN ANALYSIS}

Porter's (1985) value chain model recognizes both primary and support activities. The way these interacted within the Corridor does not find a homogeneous response since each case study shows its own characteristics and dynamics. Table 8.1 illustrates a summary of these element related to each case study and the explanation of this assessment follows.

Table 8.1: Value Chain Analysis - Relevance of Porters' Elements to Case Studies

\begin{tabular}{|l|l|l|l|l|l|}
\hline Value Chain Elements to Case Studies & $\mathbf{1}$ & $\mathbf{2}$ & $\mathbf{3}$ & $\mathbf{4}$ & $\mathbf{5}$ \\
\hline Primary Activities: & & & & & \\
\hline Inbound Logistics & $* * *$ & $*$ & $* *$ & $*$ & $*$ \\
\hline Operations management & $* * *$ & $* * *$ & $* * *$ & $* * *$ & $* *$ \\
\hline Outbound Logistics & $* *$ & $* *$ & $* *$ & $*$ & $*$ \\
\hline Marketing and Sales - access to markets, supply chain & $* * *$ & $* * *$ & $* * *$ & $* * *$ & $*$ \\
\hline Support Activities: & & & & & \\
\hline Firm's Infrastructure & $* * *$ & $* *$ & $* *$ & $* *$ & $* *$ \\
\hline Human Resources - knowledge and skills & $* *$ & $* *$ & $* * *$ & $* *$ & $* * *$ \\
\hline Technology Development & $* *$ & $* *$ & $*$ & $* *$ & $* *$ \\
\hline Procurement transformation options & $*$ & $* *$ & $*$ & $* *$ & $* * *$ \\
\hline Where $*$ low, $* *=$ medium, $* * *=$ High & & & &
\end{tabular}

Case Study 1 identifies itself with Inbound Logistics (bunkers / turn-around-time of containers); operations (towing / cargo handling / hiring of vessels / combined operations around the world); and Marketing and Sales (better transit-times / more accurate scheduling / broader scope of services / expanding intermodal transportation / greater sailing frequency).

The firm's infrastructure along with an upgrade of the overall technological profile of the JV, possibly are the two most relevant contributions of Porter's value chain model (2001) to support activities to better understand the value of the Corridor. While the first embodies around-theworld offices, trucking equipment, warehouses, transfer facilities, and container manufacturing 
premises in distant geographies (which provides them with a tangible competitive advantage), the second focuses on more comprehensible and integrating information systems technology. Unlike Case Study 5, traditional knowledge was not here a dominant factor despite human resources played a role of importance in letting the global firms better interpret the regional market variables and have access to it through the regional executives.

It becomes apparent that a combination of Porter's primary and support activities held a vital influence in the organizational transformation of these companies to face the globalization. The Corridor seems to have facilitated some of the generic strategies and create the necessary conditions for knowledge to spread, leadership to emerge, to bridge gaps in mutual understanding, and business transformation to take place based on both achieving larger economies of scale and gaining competitive advantage.

Inbound Logistics had a lower relative relevance in Case Study 2 as only part of the land-bridge design involved maritime transport and therefore a close tracking of the containers from the moment they were delivered to the customers (which only happened in Chile and for a limited period of time). However, the other two primary activities became as relevant as in Case Study 1 as both Operations (coordination of ship-rail-truck mode / standardization of equipment / rolling stock / ground transportation contracting and equipment) and Marketing and Sales (enlarged sales point / faster transit-times / better frequency of sailings / faster round-trip) were critical parts of their business transformation process.

A certain degree of traditional knowledge within the Human Resources area is one specific support activity that stood out on the part of the railway segment since this business transformation project found no parallel or form/type of imitation for a long period of time after it was launched. Traditional knowledge in this sense refers to individual tacit or uncodified knowledge that is transferred at the individual level through HR practices of mentoring, coaching and facilitation of knowledge exchange between people. This might well indicate that traditional knowledge acted as a barrier for new entrants until such knowledge leaked and spread toward new players. Naturally, both the Firm's Infrastructure and deployed Technology also were of 
relevance in view of the project's internationalization level (Europe / Middle East / North Africa / South America) and the successful interaction of their information systems technology.

The Corridor facilitated not only the companies to interact and benefit from each other by sharing knowledge and promoting a strong leadership trend to prevail over those holding lesser knowledge or market positioning to become influential, but it also brokered the formation and development of a number of CoPs aiming at fostering innovation and business transformation. Such a process brought about in some cases a high degree of innovation, despite initial doubts or a given level of skepticism, this eventually led to an effective business transformation process.

Case Study 3 shows a different profile with respect to Porter's (2001) value chain. Inbound logistics represented a complete commercial responsibility over the containers turn-around-times in view of ALL's international commercial structure, while it only held operational (cost-based impact) involvement and therefore responsibility over the regional market - leaving the ocean leg to the contracted ship-owners. Outbound logistics were also a factor since being a regional railway company they had to service many export-oriented customers only at domestic level. Therefore, this is a distinctive aspect if compared to all the other case studies. Operations as well as marketing and sales were as highly relevant as before, especially when considering both their extensive regional operations and broadened overseas sales points together with a conceptual understanding of the importance of exerting control on the cargo-routing decision making. These two activities provided ALL with an interesting degree of innovation which eventually led the company to a substantial business transformation by re-orienting their understanding of the surrounding business context.

A rather high level of traditional knowledge on the part of both the executives and lower layer staff made the human resources field to stand out as the most relevant among the support activities. Once again, both the existing level of traditional knowledge and its retention by the railway company seem to have played a critical role in deterring overseas new entrants from taking control or limiting their scope of action. Contrary to the previous case studies, IT systems were not that relevant in view of the company's pre-existing satellite systems that made new investment or upgrades unnecessary. Regional information systems proved to work well before 
ALL joined the Corridor and developed this project - leaving ship-shore interface in the hands of the shipping companies who by then proved to be in a better position to do so more effectively than any other party. The firm's infrastructure became of paramount importance in achieving larger economies of scale and gaining competitive advantage by moving toward attaining a tighter control over the cargo routing decision-making - an aspect no operator could have undertaken seriously without committing with an appropriate international network.

The Corridor might at this point be attributed to have created the necessary awareness in the minds of ALL executives and operational staff because they had to engage in a profound reengineering process if they wanted to have a chance to face what the globalization wave might bring to the region. Uncertainty seems to have exerted a great deal of pressure on the company's decision makers to interact and share knowledge at the roundtable forums - where the germ of innovation seems to have spread successfully.

Case Study 4 differs from the previous case studies in that leadership was a more dominant issue than anything else since the main stakeholder neither lacked financial capital nor was unaware of the negative impacts the globalization wave might introduce to the region. Strong leadership was always evident even before Murchison joined P\&O Ports (a world-class operator) and loomed large from the moment they were compelled to retreat and start all over again just 70km away from where they had exerted an almost undisputed leadership for so many years.

In this particular case, ATZ’s traditional knowledge (market / regional customers / culture / fidelity / uses and customs) was clearly not enough to deter P\&O Ports from controlling Argentina's main port stevedoring operations - which suggests there might be a link between traditional knowledge and a lower-relative standardization degree ${ }^{84}$. However, it seems to have played a decisive role in capturing and therefore exerting control over a significant share of cargoes that used to naturally fall within Buenos Aires’ port hinterland.

\footnotetext{
${ }^{84}$ The higher the standardization of the operations (containers / customs / roads / bridges / controls / etc) the lesser relevance traditional knowledge has. The lesser standardization the higher importance traditional knowledge takes especially in the far hinterland where improvisation along with uses and customs play a clear entry barrier for those global companies holding higher degrees of global standardization.
} 
The concept of leadership is immersed into Porter's (2001) value chain model in that Operations and Marketing and Sales turned out to be instrumental to first detain and later counterbalance an overwhelming superiority based on P\&O’s brutal economies of scale, state-of-the art technology, almost unlimited financial resources, and globally-oriented human resources. It becomes apparent that $\mathrm{P} \& \mathrm{O}$ could have easily surpassed ATZ's regional influence within its very same hinterland in view of their supremacy in both the primary and support activities. However, ATZ's leadership and their regionally-focused traditional knowledge dynamics seem to have delivered value to their strategy and marked a clear line dividing the areas and segments each player could best manage.

The Corridor was useful in bringing closer two parties that were active in different though complementing segments (port operations and foodstuff exporter) with apparently no common interests other than the typical shipper-port relationship. ATZ was not particularly interested in capturing anyone else's ideas or in getting transferred a specific piece of knowledge for them to internalize and project to the future. This contracts with the previous case studies and to what most stakeholders have done while in the roundtable forums. ATZ already possessed traditional knowledge, vision, talent, experience, and a truly genuine wish to keep leading the way they did for so long in the recent past. Consequently it was in search of a strategic partner who could provide them with a substantial cargo volume throughout the year as well as financial support to back up a challenging project and make it sustainable toward the future.

Case Study 5 embodied two distinctive particulars: a) a typical scenario where traditional knowledge became critical to the success of the barging project; and b) an astonishing lack of leadership and apparent passiveness on the part of the steel manufacturing company's management. The Corridor can be attributed to have created the needed ambience for a number of players to interact and essay different possible alternatives aiming at producing a potential business transformation scenario. Clearly, knowledge sharing and transfer, leadership, cooperation, and collaboration, all played their part in modifying the on-going market conditions and develop a tailor-made solution in the form of a project. The support activities were also of vital importance in that Human Resources became essential in the transfer of knowledge amongst the stakeholders (mainly from those holding knowledge to those who at the outset showed 
passive or indifferent), this being an aspect that was not present in the previous cases. The Firm's Infrastructure also was critical to this project as its optimization and continuous betterment truly reflected the very need of the project in itself, this being an aspect that finds coincidences with the previous cases.

It becomes apparent that the value chain model provides a useful framework to help interpret how things were changing in these cases. Both the primary and support activities described in the case studies, and the fact that the Corridor played a decisive role in not only allowing knowledge to spread and cross-business interaction to take place but also to foster and broker them through the roundtable forums as CoPs, played an important role. The value chain framework allows us to see what value elements each participant can recognize as a gap that they might fill or seek from other JV partners. This helps to explain the particular constellation of collaborating firms that emerge.

\section{4 - CROSS-CASE STUDY LEADERSHIP ANALYSIS}

The 5 case studies will now be analyzed here from a leadership perspective by assuming that management and leadership cannot occur in the same person (Bennis \& Nanus 1985; Zaleznik 1977; pp62-70) or as argued by Yukl (2002 pp5-6) and that the two styles value different functions and each has distinct concerns. It also considers as valid Bass \& Stogdill's (1990 pp383-86) view that a transformational style does not replace a transactional style but that it may complement it.

The impact of how trust was generated and developed, as noted in Chapter 3 section 3.8 relating to culture, also deserves some attention as it impacts and is impacted upon by leadership styles and resulting transactional or transformational leadership approaches taken by case study participants. This forms a backdrop to explaining how leadership styles developed and how trust impacted upon outcomes. Figure 8.2 illustrates a model adapted from (Walker, Bourne and Rowlinson, 2008, p80). Calculative trust moved towards relational trust allowing leadership styles as indicated. Trust was built from initially being based upon integrity of actions, then over time in each of these case study transformational projects benevolence towards parties was tested and when both integrity and benevolence was well tested and accepted at the mature stage of 
these projects the main trust issue was ability of individuals involved and organizational ability to deliver promised results.

While Figure 8.2 implies a uniformity of trust within entities, various parties held different perceptions of trust that also varied over time. However, Figure 8.2 does provide a useful background to understanding the degrees of transactional and transformational leadership exercised by JV parties.

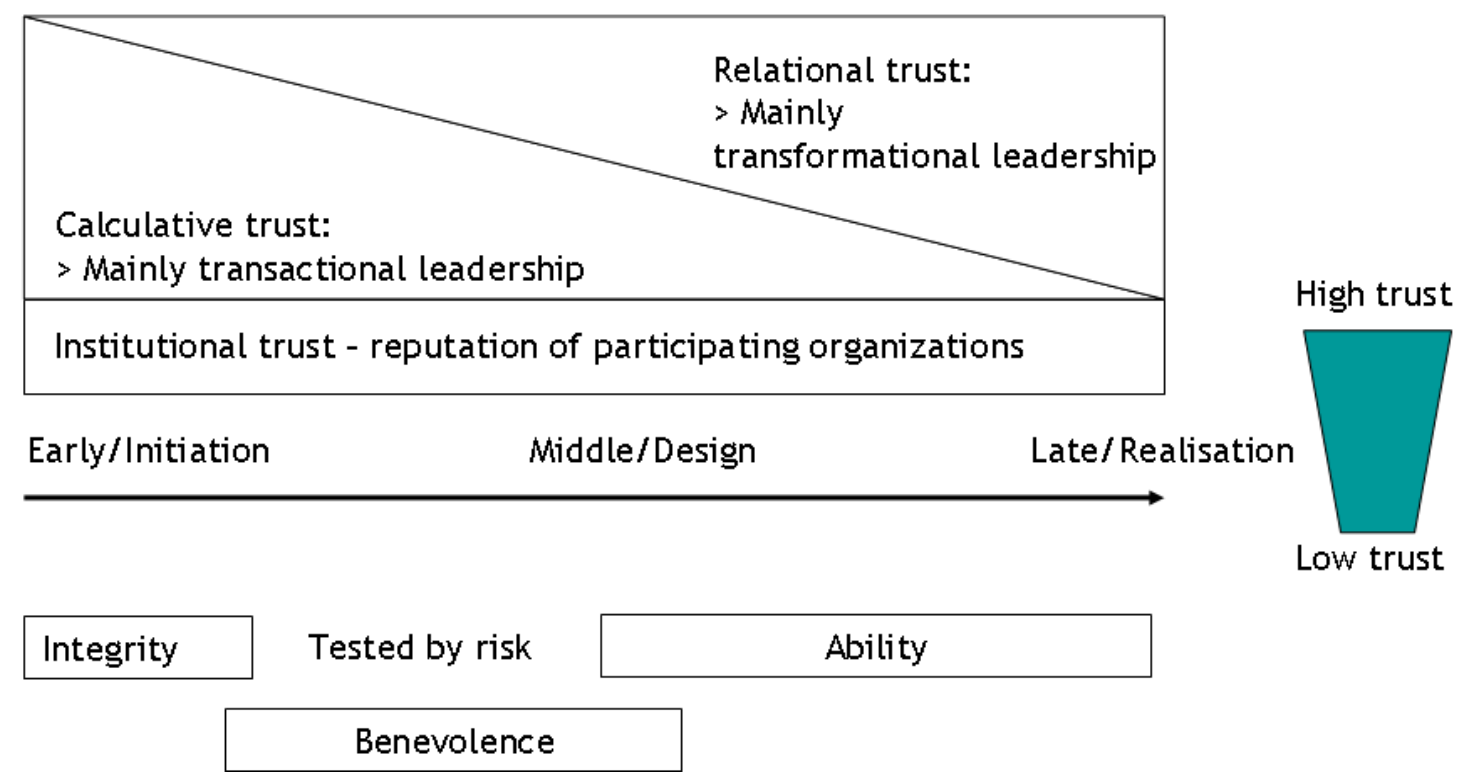

Figure 8.2 - Trust and Leadership Form and Style Influences

Case Study 1 involves a number of shipping companies that by the time they initiated negotiations showed a negligible degree of vertical integration - an aspect which later they changed in order to exert a tighter control on demand and consequently to achieve larger economies of scale. This case study entails the formation of a JV by a number of regional companies that foresaw at a given point in time that they needed to drastically reengineer their organizational structure to lower operational costs and gain overall efficiencies to face worldclass contenders. Both transformational and transactional leadership styles were present as every organization had something to profit from one another, which meant that all participants had something to give up as well. Contribution details for managerial resources, information 
technology means, corporate internal communications, suppliers’ optimization, and standardization of operational procedures were just a few of a long list of functions that needed consensus amongst the members. Those who held the largest structure and market positioning (Zim) as well as the deepest regional knowledge on customers and systems (Maruba) can be regarded as putting into a practice a Transformational leadership style while the other two became Transactional followers.

Case Study 2 also found its two main stakeholders (Zim \& BAP) showing too little vertical integration at the outset of the project. However, this tended to change rapidly in view that this very project was based on a consistent vertical integration process by which each transport mode provider was going to coordinate and optimize its operations with the other two to make the landbridge project work (ship-truck-rail mode land-bridge). Further to a growing vertical integration strategy, this project entailed a market development strategy by expanding sales points all over Europe, part of the Middle East, and the entire North Africa for which a transformational leadership seems to have played a critical role. Once more such a role was taken by the company holding the larger structure and more solid market positioning (Zim) together with a more versatile attitude toward facing volatile or uncertain scenarios - suggesting this company may have taken advantage of its already existing international network wherefrom knowledge could have been absorbed and duly imported to the benefit of both stakeholders and project.

The railway company adopted the role of a follower, though was also active in negotiating many operational aspects that demanded a certain level of good management - suggesting perhaps more a Transactional leadership type showing a certain degree of knowledge-based power distance. So the first 2 case studies look similar with respect to both the leadership style they put into practice and the variables they were based on.

Contrary to the previous case studies, the $3^{\text {rd }}$ one shows an aggressive horizontal integration strategy on the part of the Brazilian railway that became regional by acquiring other railway networks both within Brazil and Argentina - with the possibility to further expand into bordering countries as well. This case study entailed not only a profound business transformation example but that also suggests that such a process would not have been possible without counting on a 
genuine leadership with Transformational particulars. A standard managerial or Transactional leadership style seems unlikely to have attained such a strategic shift—especially when also considering this firm's international marketing strategy which led them to exert a tighter control on the demand to challenge global ship-owning companies.

Case Study 4 also shows a clear Transformational leadership style in that ATZ was determined to lead even before breaking its JV with P\&O Ports and initiate a brand new project up-river. However and contrary to the previous case studies, ATZ became active by engaging in an innovative horizontal integration strategy by joining Sadia as well as a growing vertical integration strategy (both forward and backward) by fixing agreements with suppliers to achieve larger economies of scale and gaining competitive advantage by better covering its vast hinterland - suggesting a strong presence of Transformational leadership style concept though most likely supplemented by the Transactional leadership style component.

Case Study 5 seems at first sight to be similar to ATZ's case in that it takes a typical Transformational leadership style to carry out a project that innovative in a rapidly changing business context. However, the number and variety of stakeholders that took part in the design and development of this project, as well as their diverse relative market positioning and structure, demanded more of the cold calculative mind of a strategist or sound manager-more likely to be reflected in a Transactional style - and not so much in a team or individual capable of performing beyond expectations. This case study seems to have required a greater leadership capability that adjusts or matches the present circumstances of the steel manufacturing firm to the potential solutions brought to the scene by a number of small knowledge-based companies indicating that a cost-leadership strategy might demand first a Transactional leadership style profile to be perhaps supplemented by a Transformational attitude at a given point of the project.

It becomes apparent that the Corridor was an entity that not only gathered different interests, views, opinions, and necessities from all over the region, but that it also can be argued to have served as a nurturing place for talent to spread, leadership to become contagious, and knowledge to foster companies to change and innovate. The uncertainty brought by the globalization triggered the establishment of the Corridor and facilitated the formation of regional roundtable 
forums. Leadership became a byproduct of the roundtable forums. The Corridor along with its CoPs can be regarded as crucial in having facilitated companies to network with one another and for leadership to emerge. Either a Transactional or Transformational style - or a combination of both - seem to have a logical connection with the firm's market positioning strategy and therefore with its infrastructure both across segments and locations.

\section{5- CROSS-CASE STUDY KNOWLEDGE MANAGEMENT ANALYSIS}

Knowledge forms a crucial basis for developing business strategy (Zack, 1999) and also has relevance to culture and behavior as an appropriate vehicle toward the creation and sharing of knowledge (Markus, 2001). Additionally and as argued by Nonaka (1991), knowledge has first a cycle or process to fulfill within an organization to be capable at a later stage to produce outcomes and help strategies to become real.

The evidence suggests that, the Nonaka (1991) SECI cycle seems to have been adopted by all the stakeholders in the five case studies in a quite similar pattern: Socialization generally came first as a realistic step for companies to know one another as well as sound and compare their respective needs and willingness to open up and cooperate with data and information - an aspect which also helped in bridging cultural and behavior gaps. Externalization followed when companies found the need to benchmark with one another and also to develop sound potential alliances and partnerships to help them share their visions on their respective business segments. It also facilitated the stakeholders to bring up and contrast their views, opinions, and understanding on what the globalization process might cause to the region and their businesses. This last aspect became the Combination stage of the SECI cycle.

Finally, Internalization resulted useful for companies to let change and innovation emerge (based on the final personalized interpretation of data and information within the organization) and consequently open new avenues for the companies to reengineer and transform their structures to better cope with the upcoming business context.

The Corridor together with the roundtable forums proved to be fruitful settings for the SECI cycle to evolve and facilitate knowledge sharing as a first instance. However and even though each of 
the case studies involved operating companies only within the logistics universe, both their receptiveness to knowledge and the complexity of the case studies individually considered posed significant barriers to a uniform or homogeneous knowledge transfer process. Although it seems to be clear at this point that the globalization process had a profound impact on the region and that uncertainty became a decisive motivational factor for the firms to gather and interact, their timing to elaborate a realistic diagnose on the situation and react accordingly showed significant differences amongst the stakeholders.

This difference may be explained by the existing diverse degree of knowledge stickiness amongst the stakeholders as well as the barriers to an effective internal knowledge transfer within their respective firms (Szulanski. 1996). While the case studies did not show any sign of heterogeneity with respect to the sources of knowledge stickiness (mostly represented by lack of motivation), the situation became different as regards the existing barriers to internal knowledge transfer. These barriers were represented by both a diverse degree of absorptive capacity on the part of the stakeholders and the causal ambiguity held by some of the case studies’ participants.

Stickiness of knowledge became a problem that deserved a continuous brokerage and follow up on the part of the Corridor with respect to the majority of the regional members that entered into any type of association with foreign companies, or held a significant international exposure (typically the JV, land-bridge, and railway examples). The waterways project also required a facilitation or brokerage assistance on the part of the Corridor in helping make the steel manufacturing company internalize the project's traditional knowledge content that was in the hands of a few small players with no comparable market positioning. Contrary to this trend, ATZ (port terminal operator) did not face any knowledge stickiness issues with their Brazilian partner despite the fact one was a consumed port operator while the other a world-class exporter (which indicates that knowledge stickiness not always had a connection with the recipient's absorptive capacity but that it may have other ingredients such as trust, vision, or just talent).

Lack of absorptive capacity and causal ambiguity played different roles in the case studies. Case Study 1 showed no lack of absorptive capacity as all the members of the JV were already shipowners who despite holding different relative market positioning and influence-they all 
comprehended well the main variables of the business and the point at which they could exert leadership. However, the regional companies tended to fall into a certain degree of causal ambiguity given perhaps by a natural resistance on the part of their management to really understand the inherent cause and effect loops. Case Study 2 also showed causal ambiguity on the part of the railway company as they probably felt they were loosing control over the demand made by a much larger, global and therefore resourceful company. Such a process might well be assumed as a natural resistance or indifference toward a new business model that proposed a new axis of leadership leading to change and innovation - aspects which often find resistance on the part of the weaker or follower party.

Case Study 3 showed a considerable degree of knowledge stickiness leading to increasing difficulties to make their newly controlled rail network in Argentina to interpret and put into practice the main operational and managerial concepts embodied in its business regional transformation. Having kept their activities and resources in a domestic environment without major regional or international exposure for so long, seemed to have hindered the Argentine leg of the business from developing a rapid absorptive capacity and adjust to the new business context.

Case Study 4 experienced neither absorptive capacity nor causal ambiguity despite the challenging and complex circumstances surrounding this project - which suggests a strong leadership on the part of ATZ and a high level of trust (knowledge transfer facilitator) amongst the partners' management. Case Study 5 entailed both a high degree of knowledge stickiness and a significant degree of resistance to internalize knowledge on the part of the steel manufacturing company. Contrary to the previous case studies, causal ambiguity became apparent amongst the steel manufacturing firm's front line employees and management who faced serious barriers toward conceptualizing the business transformation model embodied in the project.

The types of knowledge transfer as explained by Dixon (2000) seem to find a perfect match for each of the case studies and therefore set interesting differences with respect to the way knowledge is passed from one team on to the other - suggesting a richness of the diversity of transformation projects the Corridor contributed to facilitate. 
Case Study 1 was the typical Serial Transfer model where Zim's global experience in setting up JVs in overseas markets was repeated and put into practice by following previously proven patterns. Case Study 2 was well represented by the Strategic Transfer type where both tacit and explicit knowledge were timely combined aiming at producing a significant business transformation. Case Study 3 falls into the Near Transfer category by which a source team transfers knowledge to a receiving team for a similar task and different location. Non-routine tacit knowledge transfer between two teams seems to have been the most appropriate to classify Case Study 4 as Far Transfer. Expert Transfer embodies the last case study in view of the infrequency and uniqueness of its tasks. Case Study 5 has some unusual knowledge transfer features. In some senses knowledge transfer was characterized by Expert Transfer in that there was a level of expertise transferred from both the Corridor experiences but also from companies involved, however, much of this was totally new to all players and so it may be more accurate to conclude that this was an example of emergence of knowledge through interaction and shared perceptions.

Whatever the degree of knowledge stickiness, recipient's absorptive capacity, and knowledge transfer type, the Corridor seemed to have played a critical role in making companies socialize first to later create the necessary conditions for knowledge to spread and transfer in different forms. The Corridor's role to facilitate interaction of parties also affected the level of trust building of relationships as illustrated in Figure 8.2. Its role throughout the transformation provided an institutional setting to maintain institutional trust as all parties were part of the same Corridor entity. It had high perceived integrity at the initiation stage and its benevolence in maintaining its positive facilitating role allowed risk testing to demonstrate that it had the ability to positively influence partners. Each partner was able to similarly develop closer trust in each other through the same mechanisms.

Even though it became apparent that business uncertainty exerted, to a lesser or greater extent, a triggering effect on the case studies' stakeholders to gather and interact, it was the Corridor and its round table forums and CoPs the ones that fostered knowledge transfer across the many logistic segments. 


\section{6- CHAPTER SUMMARY}

The Corridor together with the roundtable forums, CoPs, and a permanent brokerage and followup of the many diverse situations seem to have created an adequate setting for the named concepts to flow and thus generate outcomes for the companies to benefit from. Porter's (1985) competitive forces and strategies was not only useful to help determining the existing similarities and differences in the case studies, but also to explain the real implications and the importance the Corridor held as a true facilitator for companies to network and produce significant business transformations.

As the globalization wave drew closer to the region, a linear dynamic growth model tended to gradually dissipate bringing about the element of uncertainty - a concept here regarded as possibly the most important triggering variable for both the Corridor to get established as a valid forum and the companies to join in search of alternative channels that helped them reengineer and face the global trend. In terms of strategic choice under uncertainty, using the Courtney et al (1997) model of four approaches, each case chose to try to shape the future; there was some evidence of reserving a right to participate and to some extent to adapt to the future. Mostly, the Corridor allowed strategy to emerge and evolve through its knowledge facilitation and scenario exploration and testing by JV partners. Porter's value chain model also became useful to better comprehend and explain the various ways companies undertook to enlarge economies of scale to become competitive and face a changing and unpredictable business scenario. Both the primary and support activities as per Porter's model marked clear differences as well as similarities amongst the case studies depending more on both the firm's market positioning and the firm's infrastructure than any other variable. These two aspects set parameters with respect to the way leadership and knowledge flew and allowed transformations to take place by letting other aspects to come up and contribute with the setting up of a number of projects (collaboration / cooperation / culture).

Even though each case study showed different types of leadership or even a combination of the two most accepted or popular currents of thought, the Corridor seems to have played a crucial role in both letting companies interact in order to build trust first, and fostering project stakeholders and outsiders to expose and cross-assess their views, opinions, and practical 
experiences at a later stage in a kind of contagious manner. Such a process turned out to be useful for companies to socialize, benchmark with one another, and become more aware of the globalization impact on the region - all done within a specialized and unrivalled corporate setting.

Recipient's absorptive capacity was improved, causal ambiguity reduced, and knowledge stickiness reduced in each case study through the Corridor's facilitating KM role and aim to generate innovative outcomes. Even though each case study showed a different reaction with respect to their knowledge needs, they were all intertwined and consequently immersed within a growing knowledge market under the auspices of the Corridor. Each case study adopted its own characteristics and showed its own degree of innovation. Perhaps one of the main attributes of the Corridor that became clear from the analysis of data is its having facilitated not only the gathering and professional interaction of those firms belonging to the same field of activity seeking to become more competitive, but also it having provided the members with the possibility to interact and benchmark with those firms of other fields.

Such a process helped the members to test, contrast, and consolidate their thoughts and opinions on a certain issue, as well as to dissipate a doubt or just discard a business transformation proposal that before interacting with others looked feasible or realistic. The case studies seem to have also shown that for companies to remain competitive within a region where its business structure is about to show great discrepancies in terms of economies of scale and resources amongst the players, some sort of vertical or horizontal integration becomes imperative to enlarge economies of scale and gain competitive advantage.

Possibly the most significant pending deliverable the Corridor failed yet to develop is given by the rather modest participation and contribution of the public sector in the roundtable forums and general business interaction. This also becomes apparent by the lack of connectivity between the regional needs in terms of business and infrastructure, and the governmental planning both at national and regional levels. Rivalry and cultural differences manifested by hidden agendas between the national governments - and to a lesser degree between federal and state governments - became a challenge for the Corridor to work on. Private businesses seemed to have overcome and reached significant business transformations by regionally integrating with a global 
projection. However, national administrations are yet to become full members to the Corridor to both contribute with and benefit from its knowledge market dynamics.

This chapter indicates that the Corridor turned out to be useful perhaps in facilitating richness and variety in a number of situations where the transforming threads became represented; mainly by collaboration, cooperation, knowledge, and leadership. Innovation occurred once these were combined with other elements such as culture, trust, and uncertainty. While this chapter has focused upon a more general management analysis of the cases and how the Corridor could be viewed in this light, it has not focused upon PM implications. The next chapter will focus on the Project Management implications for the Corridor in the future as a way to improve what has been developed so far. 


\section{CORRIDOR'S PROJECT MANAGEMENT IMPROVEMENT NEEDS}

\section{1 - INTRODUCTION}

This chapter briefly addresses, from a PM perspective, those aspects where the Corridor project needs improvement to better face business scenarios going through turbulent times. It starts by highlighting the way the absence of the public sector during the early stages of the Corridor has impacted on the potential deliverables as well as on the Corridor's CoPs and brokers. It draws upon concepts of stakeholder engagement; refer to Bourne and Walker (2005) for more on this PM perspective in terms of the need to generate value and benefits to players in JV's as well as their clients and customers. It also draws upon theory on trust and commitment not only for building collaborative relationships but also for high levels of input into enabling effective KM and situated learning (as discussed in Section 3.7).

The bureaucracy is yet to join the Corridor and could add value through their government-togovernment and region-to-region influence. An important gap in the composition of Corridor players was the exclusion of public sector players at a national or regional level. It remains to be seen whether that could or would have been an asset or liability to the way that the Corridor operated and it represents a gap in our understanding how the case study's project deliverables could have been improved or harmed if public sector players actively joined the Corridor and addressed concepts such as power distance, decision-making timing, and project coordination through integration management. This section further continues with a brief analysis of the impact that the public sector could have had on Porter's (1985) competitive forces to end with some implications related to Nonaka's (1998) SECI cycle as well as on performance monitoring aspects. The chapter ends with a chapter summary

\section{2 - PUBLIC SECTOR: POTENTIAL PROS AND CONS}

Both the roundtable forums and the various CoPs that were created to later evolve as a supporting mechanism towards an effective regional networking were based almost entirely on the participation of private stakeholders. The public sector played a minor and secondary role as it had never really become involved in the process despite the fact the very survival of an entire economic sector was pretty much at stake. There was no active participation of the public sector 
in the form of official representatives, delegates, or even observers other than occasionally a port representative who as a secondary stakeholder limited himself to attend the forums and take notes to possibly draw up an internal report on the minutes.

From a project management perspective it may be affirmed that having counted on the active presence and participation of the public sector's various agencies at an early stage of the Corridor formation (road and rail transportation agencies / waterways and ports secretariats / air transportation agency / coast guard / frontier police / customs house / environmental department / strategic planning ministry / etc) both at federal and state levels, might have proved to be an additional challenge to overcome by the Corridor in terms of project interface coordination through integration management. As projects become more complex, drawing on knowledge and skills from more areas of expertise - and thus, more subunits of the parent organization as well as more outsiders - the problem of coordinating multidisciplinary teams becomes more troublesome (Meredith \& Mantel 2009, p267). And together with it, uncertainty tends to increase in a business context where stakeholders desperately looked for responses that placed them beyond these uncertainties.

If government players at national and regional levels would have actively contributed to the Corridor's facilitation effort so that high levels of trust and commitment was generated, then some of the obstacles of dealing with uncertainty about government policy or its reaction to various initiatives may have been reduced. This could have moved strategy from having to deal with the most complex situations described by Courtney et al (1997) at the $4^{\text {th }}$ level of ambiguity to one where more stable plans could have been made, for example at level 3 where a range of futures can be explored using scenarios or even level 2 where a limited number of potential futures can be identified. Further, greater knowledge exchange from this sector could have proved useful in reducing uncertainty. However, risk management involving government players can prove to be a two-edged sword as I later elaborate upon. 
Both the federal and state agencies normally plan their infrastructure investments within a 5 to 10 years horizon, which would have turned out to be possibly useless and even negative when compared to the stakeholders' by then current needs and strategic urgencies in a context of gross turbulence and uncertainty. The concept of survival would have possibly held very different meanings for a private firm and a state or federal agency. As discussed earlier in Section 3.2, different uncertainty types require different strategic responses (Courtney et al., 1997).

Government instrumentalities, particularly long established ones such as that existing in Latin America at the time when the Corridor was being developed, tended to be bureaucratic and lacked the flexibility in outlook to focus on benefit realized rather than ordained processes that had been long prescribed. This is not unusual and as Hodgson (2004) argues, this rigid mindset is apt to transfer the 'iron triangle' norms in being considered 'good or best PM practice' when agility may be needed. Others have also drawn attention to this potential scenario (Styhre, 2006). The concern, or fear, that I observed was that while there could be distinct advantages for government inclusion in the Corridor, it may have come at a cost of required highly processdriven mechanistic and processes that were favored at the time by government instrumentalities.

Additionally, there is a considerable power distance in South America between the public and private sector as discussed in Section 3.8 of the literature review. This can be better visualized by the existing differences between those people who lay down the rules and the ones who obey them, with little intermediate steps - a scenario that in multidisciplinary teams within a project setting might well become counterproductive. Rivalry amongst the regional countries’ authorities to favor one region's development plan against another one - although never experienced so far at this level - might well be expected to take place and consequently to lead to a slowdown process on the private sector as far as business transformation is concerned.

Following Porter's (1985) analysis with respect to the role the competitive forces play for companies to decide on expansion and investment, it becomes apparent that the concepts of Entry of New Competitors and Threat of Substitutes are aspects very much identified with a short term scenario and not so much with a longer term scope of action as it would be more appropriate if a federal or sate agency were to be involved. 
However, a shorter term scope of action on the part of the public sector might well have had a direct impact on the Bargaining Power of Buyers and Suppliers as well as the Rivalry Amongst Existing Competitors-for example in expanding an existing port terminal, allowing a free zone ${ }^{85}$ to operate, or opening border passages between two countries to promote regional trade. Thus, depending on the on-going circumstances, such an impact could have been a constructive element in that it would have helped stakeholders anticipate a given scenario and therefore formulate better focused strategies that ultimately would bring about a kind of transformation under a lower level of uncertainty. Conversely, a deeper knowledge of the private stakeholders' plans, visions, and needs might well contribute to better planning in physical infrastructure projects by the federal agencies.

The modest or limited participation of the public sector in the Corridor's networking scheme has provided the Corridor stakeholders with practically no support on aspects pertaining to policy formulation which would have helped them foresee some upcoming developments as to let them adjust or coordinate their strategies within a pre-defined framework. It also becomes apparent that if a truly active participation of the public sector had taken place from the outset of the Corridor project, there would have been a significant gap between the private sector's short run needs and the public sector's long term focus - given their different priorities as well as exposure degrees to the globalization impact. One might infer here that various agencies might have felt tempted to regulate the incipient business transformation process with perhaps an excess of jealousy by laying down an endless set of rules and regulations as a way to intend controlling the market variables as they did in the recent past. Notwithstanding, a certain level of internal competition amongst the sate and federal agencies might have also led to a general betterment of the system.

Despite the potential negative effects the public sector could have had on the early stages of the project, it is been a pending issue for the Corridor to effectively engage the various federal and state agencies to actively participate in the roundtable forums as well as to get them integrated in

\footnotetext{
${ }^{85}$ A Free Zone is a designated area where storage of imported goods, physical transformation to goods, and tax deferment schemes are allowed to promote the development of a specific sector or logistic corridor.
} 
the various CoPs. It seems to be highly desirable for the Corridor to also recruit public officials capable of bringing their long-term views as well as many relevant agencies as possible to help the companies set their strategies in a more predictable business environment in terms of infrastructure development and policy formulation. Policy formulation by federal agencies might well help private operators to raise entry barriers to their respective segments and make their businesses less vulnerable to future trends. It is conceivable that government players, once their interests would be aroused for fostering local or regional industry, that they may take on a role akin to that of project sponsor or champion as described in Section 3.9.

It becomes apparent that the Corridor project was born as the globalization threatened many companies to interrupt the kind of linear growth they had somehow become used to enjoying thanks to some governmental protection embodied in an overall regional protectionist trend. Having counted on an active, regular, and decisive participation of the public sector from the very beginning of the Corridor project, would have likely become deeply counterproductive. As the Corridor can be regarded as a mother project that continuously nestles a rotating number of subprojects going through the four stages of a project's life cycle (while the Corridor yet keeps away from its phase-out stage), it becomes highly advisable for the Corridor and their stakeholders to recruit more governmental officials and agencies that can ultimately bring value to every stage of a project's life cycle from conception to termination or phase-out. Active public sector agency participation could also be useful for them to formulate shorter term plans as well as the private sector adjusting their decisions' timing to what the public sector might deliver. Should the Corridor succeed in doing this, it is likely that the projects' deliverables and sustainability might tend to substantially increase in the future.

\section{3 - CONSIDERATIONS OF KNOWLEDGE AND PERFORMANCE}

Another aspect of relevance where the Corridor should do better in future is also related to public sector participation. However in this regard it involves the SECI cycle as a way of softening the public officials' natural resistance to interact and contribute data and information that might be beneficial in shaping new alliances or setting new business trends. 
The Corridor has not only failed in recruiting public officials and institutions to join the roundtable forums but also has been unsuccessful in making their brokers truly understand the importance of gaining the public sector's support and contribution during each stage of a project's life cycle. There was an almost complete absence, as a by-product of this failure, of key micro or macro economic performance indicators (KPI) in particular being required of the Corridor's brokers and the stakeholders as a consequence of possibly having no such entity or public organism to report to. Thus a potential focus for optimization may have been lost.

Even though one may expect the Corridor to have set their own performance parameters, it is more likely that a Corridor-like organization had felt more compelled to report to the public sector in the form of KPIs. Projects were regarded as successful or unsuccessful as they move forward and overcome the various stages until accomplishing some sort of integration or alliance. Gaining the public sector's participation from an earlier stage would have possibly compelled the Corridor to define a number of leading activities and processes to later set some basic standards that could have proved what the Corridor's contribution to the economy was, and thus to gain the support of the government agencies.

From a productivity standpoint the Corridor showed a certain lag in the decision-making process once the globalization wave had passed. This experience is similar to that of many large firms when intending to define which projects to approve and which ones to discard during stable times. This was partly attributable to the absence of uncertainty acting as a truly triggering feature - which was precisely what gave birth to the Corridor in the first place: many more projects than management expected; inconsistent determination of benefits; competing projects; interesting projects that did not contribute to the overall strategy; projects whose costs exceeded their benefits; and lack of tracking by the Corridor executives. This is similar to project program management strategic decision making where projects are more effectively chosen to be undertaken based on a more coherent strategy. This has been a subject of much discussion in emerging views of how program project management is developing (Archer and Ghasemzadeh, 1999; Norrie, 2006; PMI, 2006a; 2006b). 
Improvement seems to be needed in all those concepts related to the absence of KPIs as a way to monitor effective outcomes, an internal benchmark procedure within the Corridor as a project quality enhancer, and a more practical relationship between the various CoPs, and the public sector as a policy setting entity and certainty builder.

\section{4 - CORRIDOR PM AND PUBLIC SECTOR ASPECTS CONCLUSIONS}

The Corridor has moved forward as a master project that has produced, and keeps on generating, an increasing number of sub-projects that show different needs and potentialities. As such it becomes apparent that it has overcome an extremely threatening scenario from the impact of the globalization wave and all the implications that has for business sustainability that has been discussed in this thesis.

This has been undertaken without the support of the public sector to help companies achieve a significant transformation process. Such a process has led companies to formulate and implement a wide array of strategies to gain competitive advantage while enlarging economies of scale that helped them face the globalization impact. Throughout this process the public sector remained as a passive witness, the Corridor, CoPs, and brokers being responsible for not having encouraged the effective incorporation of both federal and state agencies.

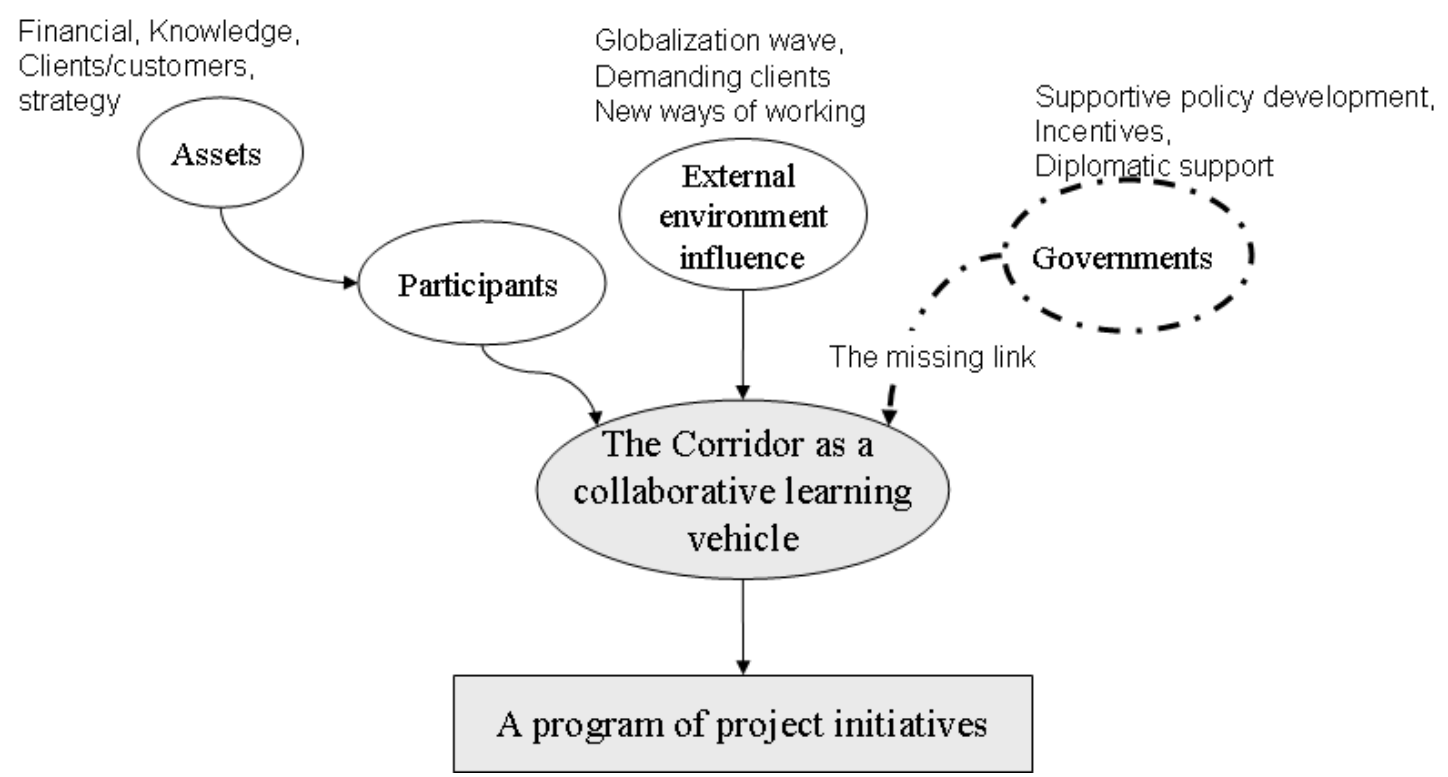

Figure 9.1: The Government Participant Missing Link 
Figure 9.1 illustrates the missing link of Government participation as an actor, along with the Corridor participants (that bring various assets and characteristics with them) and the external environmental influences (that have also been illustrated through the case study chapters context and literature review chapters). Government participation presents a missing link that could have on the one hand added strength to the facilitation of the Corridor and on the other hand created some tensions that would present challenges to the Corridor.

The same immediateness and urgency that dominated the corporate world during the early 1990s, seems to have also taken its toll on the Corridor as the public authorities were practically overlooked, if not ignored, during this period. The public sector might have provided the various logistic segments with better tools to struggle against the uncertainty of the external environment and therefore achieve better outcomes in their respective projects. For example they could have offered developmental policy support, incentives or diplomatic intervention and support at global trade talks and negotiations.

However, early participation might have also turned out to be highly detrimental for the Corridor project as a whole in view of the response speed the ongoing business context demanded vis-à-vis the often slow pace decision making by the governments. Although this is a territory where no experience has so far been registered, one can only speculate on the outcomes and various situations. Notwithstanding that speculation, which is not suitable to be elaborated upon without presenting supporting evidence, it becomes apparent that the conditions for the public sector to actively join the Corridor project seem to be nowadays far more suitable for a proper adjustment and interaction of both the public and private industry sectors.

Without too much uncertainty on the horizon ${ }^{86}$, as compared to the early turbulence of the globalization wave discussed earlier, and having left the globalization impact well behind, it is likely that the Corridor can only benefit from the public sector participation in that it might bring about new knowledge dimensions, help setting standards, and give stakeholders a sense of

\footnotetext{
${ }^{86}$ The unfolding global economic recession that is unfolding however at the time of writing this thesis could and does present a new and different kind of turbulence and uncertainty should be acknowledged as potential modifying this assertion, particularly if government financial guarantees or facilitating financing of projects becomes an essential enabler..
} 
support and planning that so far was never experienced. Projects growing under the Corridor's umbrella are currently more likely to find regional governmental agencies offering helpful responses to enquiries and requests for knowledge and to create more certainty in solving issues.

\section{5 - CHAPTER SUMMARY}

This chapter focused upon an important issue relating to those aspects, from a project management perspective, where the Corridor could perhaps improve its structure and actions to more fully engage with the government sector.

It started with discussions of the advantages and disadvantages of a more intense public sector involvement given the Corridor's history. The added support and knowledge that governments could have contributed was identified and a model was presented in Figure 9.3 to illustrate the perceived potential gap. This now naturally leads to the final chapter of this thesis. 


\section{FINAL CONCLUSIONS}

\section{1 - INTRODUCTION}

This final chapter commences by describing the most important structural aspects arising out of the case studies and presented theory, that leads the reader to better conceptualize how the Corridor project became a viable undertaking in facilitating positive transformational change in partner businesses. It continues with a summary of the principal general conclusions by addressing each research question posed in Section 1.4 and Chapter 5 thus summarizing and bringing a number of concrete responses that find common ground in all the 5 of the case studies and presented theory. This is followed by acknowledgement of this thesis' limitations with respect to aspects that were not included in this thesis for scoping reasons as well as limitations relating to the author's own views and experience. It also highlights some areas deserving further study as well as the potential for other Corridor-like type of organizations to develop in the future.

\section{2 - BUSINESS TRANSFORMATION CONCLUSIONS}

Artto and Kujala (2008) identify management of a business network and its interface between managing a project network as an emerging and valid field of PM study. This thesis is based on just such an aspect of 'project work'. It therefore validly falls within the scope of a research study in the PM discipline. Additionally, as highlighted in Chapter 3 Section 3.8, the Corridor could be seen as a form of Project Management Office (PMO) in the way that it facilitated joint ventures and other forms of collaboration and business transformation. Chapter 4 focused upon the Corridor as a knowledge management agency that presents it as a facilitator of knowledge work in a PM knowledge chain setting. As noted in Section 1.8, Winter et al (2006, p642) discuss emerging trends in PM research and highlight three directions of research for practice that is needed that relates specifically to business transformation type projects and to the approach undertaken in this thesis. These are: Direction 2 (projects as social processes); Direction 3 (value creation as the prime focus); and Direction 4 (needing broader conceptualizations of projects having multiple purposes). They also stress in their Direction 5 a need for PM theory in practice through practitioners being reflective learners. This research work meets these criteria because it investigated the Corridor as a social process. It is also conceptualized not only as a transport 
logistics enhancer (which is one lens through which it may be viewed) but as a program of projects that facilitated business transformations. It also draws upon the disciplines of economics, knowledge management, business process re-engineering through business transformation in addition to supply and value chain analysis all within a trans-national context. Thus it addresses Research Direction 3 and 4. Finally, in undertaking this research as a series of selected illustrative case studies in which the researcher was a participant observer, as recommended by Coghlan (2001), it undertakes deeply contextual research work in an emerging PM area addressing the Winter et al. (2006, p642) Research Direction 5 of the author of the research being a reflective practitioner. The basis of this research as being appropriate as a topic for a DPM thesis is therefore established. This was discussed in Section 5.1 in the research aims, propositions and questions. Finally, as Hodgson and Cicmil (2006b; 2006a) argue, the 'lived experience' of project managers and those engaged in project work is an under-researched and much needed are for PM research. By being a participant observer and reflective practitioner, I have been able to draw upon deep and rich insights from my experience and thus add a valuable contribution to PM research.

This thesis has been developed by considering a number of variables, identified as constituting principal elements of the five case studies as well as being the main pillars upon which the Corridor project evolved to produce (in different combinations) similar outcomes across each of the case study transformation projects. These pillars (collaboration, cooperation, knowledge sharing and transfer, leadership, and culture) all play different roles and exert a specific influence on case studies presented.

It becomes apparent from discussion of the thesis' project context presented in Chapter 2 that the globalization phenomenon has found a fertile ground in South America. This phenomenon strongly impacted its business structures in view of the region's unawareness of the global trends as well as the belief by its business community that a kind of linear growth business context could be sustainable without having to engage in business transformation, reengineering, or integration strategies of any kind. Chapter 2 provided the background context to understand how during all this process the above mentioned variables became critical for the Corridor to evolve as a mother project and let a growing number of sub-projects to also evolve and produce innovative 
project deliverables within the logistic discipline. It can be affirmed that a profound business transformation process has taken place across the region, uncertainty being the greatest motivator and the potential threat brought by globalization the real triggering element. These two concepts have undoubtedly contributed to the Corridor to become established and allow companies to join it in a defensive action. There were a number of structural aspects that transpire from both the case studies results and theory presented in Chapter 3 and 4 that brought about a greater number of variables to interact and facilitate this large business transformation to take place.

Table 10.1 summarizes ten main business transformation drivers/inhibitors through the Corridor's facilitation role together with brief comments drawn from the analysis of the case studies.

Table 10.1: Corridor: Transformation Facilitation Role Drivers and Inhibitors

\begin{tabular}{|c|c|}
\hline Driver/Inhibitor & Comments \\
\hline $\begin{array}{l}\text { 1. Lack of strategic } \\
\text { vision by companies in } \\
\text { this region } \\
\text { INHIBITOR }\end{array}$ & $\begin{array}{l}\text { This tended to make them favor a short-term or tactical financial } \\
\text { transaction scenario instead of projecting themselves toward a longer term } \\
\text { integration business context } \\
\text { Corporate decisions showed no concern for the long run but and } \\
\text { focused on very specific short run yielding business niches with little or no } \\
\text { strategic value. }\end{array}$ \\
\hline $\begin{array}{l}\text { 2. Slowness to react to } \\
\text { the emerging global } \\
\text { trends } \\
\text { INHIBITOR }\end{array}$ & $\begin{array}{l}\text { This related to the need for regional firms to achieve larger economies } \\
\text { of scale and to gain competitive advantage to better face the arrival of } \\
\text { much larger and more resourceful foreign-based operators. } \\
\text { A clear example of what globalization was going to bring concerned } \\
\text { those global shipping operators that held a much larger carrying capacity } \\
\text { along with a much lower slot cost than their global competitors who also } \\
\text { had a much more advanced technological and managerial profile that } \\
\text { tended to establish an unbridgeable operational gap. }\end{array}$ \\
\hline $\begin{array}{l}\text { 3. False expectations } \\
\text { about the region's } \\
\text { economic growth } \\
\text { sustainability } \\
\text { INITIALLY AN } \\
\text { INHIBITOR THEN A } \\
\text { DRIVER }\end{array}$ & $\begin{array}{l}\text { There was an erroneous perception on the speed and aggressiveness } \\
\text { with which the globalization phenomena hit and expanded across the } \\
\text { region. } \\
\text { This process had a dual impact. It was first manifested by complacency } \\
\text { followed by a realization that a strategy for survival needed to be } \\
\text { developed. The later made cooperation and collaboration an urgent } \\
\text { necessity to spread across the regional corporate world. This realization } \\
\text { contributed to gradually erasing counterproductive corporate attitudes and } \\
\text { personal feelings such as rivalry, secrecy, distrust, pride, jealousy, } \\
\text { complacency, and hidden agendas. }\end{array}$ \\
\hline $\begin{array}{l}\text { 4. Excess of confidence } \\
\text { in the regional market } \\
\text { protective laws. }\end{array}$ & $\begin{array}{l}\text { This resulted in failure to realize the global operators' structural } \\
\text { advantage, strategic focus, and political power to create their own legal } \\
\text { framework to operate in. The regional operators failed to visualize how } \\
\text { low in fact their businesses' entry barriers turned out to be, as well as how } \\
\text { overwhelming the difference in operational scales were between them and } \\
\text { the global operators. }\end{array}$ \\
\hline
\end{tabular}




\begin{tabular}{|c|c|}
\hline $\begin{array}{l}\text { 5.Lack of stakeholders' } \\
\text { inhibition to cooperate } \\
\text { when necessary } \\
\text { A DRIVER }\end{array}$ & $\begin{array}{l}\text { This seemed to have played in favor of transforming the Corridor } \\
\text { project into a win-win project generator entity by means of cooperation } \\
\text { and collaboration, suggesting that information complexity and its attached } \\
\text { potential knowledge viscosity did not present barriers that were too high to } \\
\text { be overcome. }\end{array}$ \\
\hline $\begin{array}{l}\text { 6. Reluctance of to ex- } \\
\text { state-owned } \\
\text { companies to embrace } \\
\text { modern management } \\
\text { practices. } \\
\text { INHIBITOR }\end{array}$ & $\begin{array}{l}\text { Lack of motivation, distrust, little or no collaboration, lack of } \\
\text { leadership, and political agendas on the part of transitional ex-state own } \\
\text { organizations were all variables that might have encouraged or accelerated } \\
\text { the arrival of foreign-based operators in some particular segments. }\end{array}$ \\
\hline $\begin{array}{l}\text { 7. Active and strategic } \\
\text { participation of both } \\
\text { CoPs and brokers } \\
\text { DRIVER }\end{array}$ & $\begin{array}{l}\text { This eased knowledge flow for the benefit of both outsiders and those } \\
\text { holding direct interests in a particular business segment. Not only was a } \\
\text { technical or professional role needed for these two players to facilitate } \\
\text { networking but also, and perhaps most importantly, their abilities to find } \\
\text { alternative ways to make knowledge stickiness, lack of absorptive } \\
\text { capacity, cultural barriers, indifference, envy, and further inhibitors to } \\
\text { dissipate and were eventually removed. }\end{array}$ \\
\hline $\begin{array}{l}\text { 8. Extremely low degree } \\
\text { of either vertical or } \\
\text { horizontal integration } \\
\text { INHIBITOR }\end{array}$ & $\begin{array}{l}\text { There was an amazing initial unawareness of how to conceptualize } \\
\text { these strategies as effective tools to face the global threat by achieving } \\
\text { larger economies of scale and gaining competitive advantage. }\end{array}$ \\
\hline $\begin{array}{l}\text { 9. A transactional } \\
\text { leadership style } \\
\text { INHIBITOR }\end{array}$ & $\begin{array}{l}\text { This turned out to be dominant throughout the years of regional linear } \\
\text { economic growth against a modest presence of a transformational } \\
\text { leadership on the part of a few companies that by then showed a certain } \\
\text { level of internationalization and therefore contact with other realities. } \\
\text { Despite this process brought about a drastic aggiornamento }{ }^{87} \text { on the part } \\
\text { of the regional players toward achieving innovative ideas and proposals. } \\
\text { The lack of transformational leadership might have been identified as a } \\
\text { regional weakness (low management entry barriers) and accelerated both } \\
\text { the Corridor formation and the coming of the global operators. }\end{array}$ \\
\hline $\begin{array}{l}\text { 10. The outstanding role } \\
\text { played by traditional } \\
\text { knowledge in } \\
\text { helping some } \\
\text { regional players } \\
\text { build defensive } \\
\text { strategies } \\
\text { DRIVER }\end{array}$ & $\begin{array}{l}\text { This factor set higher entry barriers for deterring global operators from } \\
\text { becoming entirely dominant in a number of segments and market places } \\
\text { within a complex and strange context for the global players as described in } \\
\text { Chapter } 2 \text { - Context. }\end{array}$ \\
\hline
\end{tabular}

The way that the above influenced the general conclusion of the thesis follows as a response to each of the research questions first posed in Chapter 1 section 1.4.

\footnotetext{
${ }^{87}$ literally meaning "bringing up to date" but according to the Wikkipedia it has a meaning of "an attempt to learn from the world and read the 'signs of the times'..see URL http://en.wikipedia.org/wiki/Ressourcement accessed on Feburary 28th 2009.
} 


\section{3 - GENERAL CONCLUSIONS}

Based on the research idea, aims, and objectives as detailed in Chapter 1 and Chapter 5 , the research proposition is that the MERCOSUR Atlantic Corridor Project can be seen as:

... an instrumental entity where regional stakeholders can find a useful system and adequate tools to formulate and implement a business reengineering strategy to achieve larger economies of scale. Two principal tools that has been demonstrably used are those used by a Project Management Office (PMO) and a CoP

As such, the system entails stakeholders to engage in a deep business transformation journey aiming at reducing existing structural asymmetries and gaining regional competitive advantage. To this end six research questions were raised and have been responded to individually and according to the findings from the case studies.

Table 10.2: Response to Research Question 1

\begin{tabular}{|l|l|}
\hline Question 1 & $\begin{array}{l}\text { What are the variables granting the Corridor project the ability to generate } \\
\text { trust first and facilitate knowledge transfer among stakeholders? }\end{array}$ \\
\hline Main Themes & $\begin{array}{l}\text { Trust facilitators and inhibitors - fear, responsiveness, collective action, } \\
\text { shared vision, CoPs }\end{array}$ \\
\hline Comments & $\begin{array}{l}\text { The Corridor became a safe haven in which knowledge could be created, } \\
\text { exchanged and used in the sense that it was a ' } b a \text { ' or special knowledge } \\
\text { management space (Nonaka and Konno, 1998; Nonaka, Toyama and } \\
\text { Konno, 2001). Knowledge work as a shared enterprise helped develop trust } \\
\text { and commitment to the business transformation. }\end{array}$ \\
\hline
\end{tabular}

The model presented in Figure 3.4 in Section 3.8 provides a useful theoretical background to view the how trust was gained and maintained by using the GLOBE cultural dimensions to visualize and measure the cultural climate. Also a model of how the Corridor developed trust was presented in Figure 8.2 in Section 8.4. Trust is considered to be a critical element for companies to interact and exchange not only data and information on a given project but also to exchange views, impressions, feelings, and visions on a given situation. Aspects such as attitudes, behaviors, codes, and most importantly cultural signals are sent out during this process for others to capture, interpret, and duly codify. Trust, as discussed in Section 3.7, is possibly the most 
important variable for a company to start seriously assessing the possibility of undertaking a strategic alliance.

However, and as it became apparent in the case studies, the main themes of trust facilitators and inhibitors turned out to be the same though the speed at which they developed and the structural circumstances became unique in essence. The globalization process had provoked most of the firms to reappraise their relationships with other firms they interacted with. While some of these were earlier considered as rivals they came together and become united in the Corridor's roundtable forums across the region. The real magnitude of the global threat became overwhelming for these companies and therefore brought about an adequate space where all these firms could actively interact, exchange visions and opinions; let cultural codes spread and work, all leading to an active and unprecedented trust generation process. This peculiar space was similar in nature to the concept of $b a$ Nonaka and Konno (1998) as a learning space where players interact and create exchange and use knowledge. It may be affirmed that the global threat brought about by globalization and its associated corporate fear have accelerated the process by which all the constituting variables of trust worked better and faster toward accomplishing trust first and consolidating an innovative business transformation later.

Even though the global threat can also be attributed to having facilitated knowledge to become shared and transferred amongst the stakeholders, it was rather the way the corporate interaction was carried out which allowed knowledge to flow that in turn generated trust and commitment. Face-to-face tacit knowledge sharing was promoted at the roundtable forums from the very outset of the Corridor project aiming at enhancing trust to emerge and flatten cultural barriers. Both the CoPs and their brokers (the Corridor facilitators who brought various players together) turned out to be critical in working out whatever asymmetry they came across in order to facilitate knowledge to flow and trust to gradually consolidate. The Corridor's aim was that the sooner this process had shown some results the more it encouraged others to feel inclined to imitate behaviors that generated those results. 
Table 10.3: Response to Research Question 2

\begin{tabular}{|l|l|}
\hline Question 2 & $\begin{array}{l}\text { How do Corridor stakeholders share vital internal information and join } \\
\text { forces to face extra-regional threats? }\end{array}$ \\
\hline Main Themes & $\begin{array}{l}\text { Shared tacit knowledge and collaboration through CoPs, JV's Partnerships } \\
\text { and Alliances - collective action, shared vision, CoPs, understanding and } \\
\text { empathy, value chain integration, sticky knowledge, Knowledge Brokers. }\end{array}$ \\
\hline Comments & $\begin{array}{l}\text { The Corridor facilitated a space where trust generated through empathy and } \\
\text { closer understanding of how players could add value to collectively gain } \\
\text { competitive advantage. }\end{array}$ \\
\hline
\end{tabular}

Section 3.8 and Chapter 4 of the literature review is particularly relevant to this question. Once the globalization was duly internalized and conceptualized as a potential threat by the stakeholders, and the interaction proved useful in allowing trust to emerge and expand, the stakeholders tended to assess what was best for their businesses in terms of alliances, partnerships, JVs, or any other type of resources combinations. This occurred either vertically or horizontally. Porter's supply chain model and competitive forces helps to conceptualize the various pros and cons stakeholders held by the time they attended the roundtable forums by addressing these potential combinations from a functional perspective as discussed in Chapter 3 and in Chapter 8

Both the Corridor as a master entity and the CoPs as knowledge enhancers and facilitators were crucial in setting up the framework within which strategic information was made available. Both entities were committed to making stakeholders aware of the importance for stakeholders to transform their structures towards gaining competitive advantage as well as introducing one another as a way to facilitate potential business synergies that could lead them to achieve larger economies of scale. However, it was up to the CoP's brokers to actively promote both the exchange of tacit and explicit knowledge amongst not only the representatives of a similar segment but also complementing or even competing segments. Such a process was of paramount importance for stakeholders to combine their assets and different types of knowledge that later brought about a number of sub-projects. The brokers also played a decisive role in dismantling project inhibitors such as those problems associated with knowledge stickiness, low absorptive capacity, anxiety, and causal ambiguity, which otherwise would have hindered a significant number of projects from being realized. This reduced the stickiness of knowledge through helping create both the motivation to learn and exchange knowledge as well prepare a fertile 
rather than barren environment in which knowledge work can take place (Szulanski and Jensen, 2004).

Table 10.4: Response to Research Question 3

\begin{tabular}{|l|l|}
\hline Question 3 & $\begin{array}{l}\text { Why do Corridor stakeholders show their current attitudes and } \\
\text { motivations towards committing themselves with partnerships, alliances } \\
\text { and joint-ventures? }\end{array}$ \\
\hline Main Themes & $\begin{array}{l}\text { Commitment to collaboration - Fear responses, competitive advantage, } \\
\text { business cooperation theory }\end{array}$ \\
\hline Comments & $\begin{array}{l}\text { The Corridor facilitated an environment where change in the prevailing } \\
\text { business paradigm was essential this also links into the concept of } \\
\text { managing business networks as a valid PM research (Artto and Kujala, } \\
\text { 2008) topic. See Chapter 2 Corridor context and Chapter 3 Sections 3.2, } \\
\text { 3.3 3.4 and 3.6 }\end{array}$ \\
\hline
\end{tabular}

Chapter 3 Sections 3.2 discussed introduced concepts and theories of competitive advantage with Figure 3.1 providing an illustration of the Corridor's role. Section 3.3 focused upon value chain theory with Section 3.4 providing a discussion of the role of the Corridor in terms of the resourced based view of the firm. The case studies clearly demonstrated that the main motivation for the stakeholders to join forces and commit to new ventures is given by the overwhelming threat posed by much larger and resourceful corporations. It may well be affirmed, considering theory presented and discussed, that it was more a matter of survival than anything else. However, it should be highlighted that even though most companies have undertaken some kind of business transformation during a relatively short period of time, it seems to have been reinforced by the Corridor as an adequate setting for the logistic companies to join and interact. Had not the Corridor existed, it is hard to imagine the way the regional logistic sector would have reacted before the arrival of global operators. One may infer at this point that a number of companies would not have had the opportunity to understand and conceptualize the magnitude of the threat and therefore sell out, becoming subcontracted, downsized, or just disappeared. Artto and Kujala (2008) have argued that managing a complex of coordinated organizations within a value chains is a legitimate and little-researched field of PM study, so this aspect is particularly salient to PM research.

The Corridor was not only useful as a master project coordinator and enhancer of alternatives, but was also a fundamental motivator or compass for firms who needed some sort of guidance and 
follow up to truly visualize how the region was going to experience the globalization impact as well as what the potential defensive options were feasible. Motivation proved to be a concept as contagious as the fear provoked by the globalization threat. The creation of the Corridor and its evolvement into a sustainable entity provided a tipping point (Gladwell, 2000; Kim and Mauborgne, 2003) which influenced the participants to use the Corridor's framework as a way to activate business process re-engineering, business transformation and other forms of change management within their organizations that enhanced productivity.

Table 10.5: Response to Research Question 4

\begin{tabular}{|l|l|}
\hline Question 4 & $\begin{array}{l}\text { How does culture play a role in easing or impeding the development of } \\
\text { the Corridor vision throughout the region? }\end{array}$ \\
\hline Main Themes & $\begin{array}{l}\text { The impact of organizational and trans-national culture on commitment } \\
\text { and knowledge work- Culture theory, trust and commitment. }\end{array}$ \\
\hline Comments & $\begin{array}{l}\text { The Corridor facilitated trust and knowledge transferred as discussed } \\
\text { earlier above. Section 3.5 on Leadership and strategy indicated how the } \\
\text { response to the threat faced by Corridor participants required a change } \\
\text { in leadership style. Section 3.6 then discussed business cooperation } \\
\text { theory which is relevant to this research question. And this led into } \\
\text { Section 3.7 where under the KM discussion relating to knowledge } \\
\text { 'stickiness' theory (Szulanski, 1996) and how culture impacts upon that } \\
\text { to provide a fertile ground to facilitate knowledge work between parties. }\end{array}$ \\
\hline
\end{tabular}

The Corridor consists of a number of companies from countries that share not only common historical roots and geographical nearness, but also values, codes, aspirations, as well as only two languages through which people can understand one another across an entire region. The relative cultural homogeneity of the region certainly helped in avoiding major constraints in the formation of new projects. Culture is not revealed here as an impediment to new undertakings but, on the contrary, it has generally proved to be an enhancer for new projects. However, certain differences related to power distance become apparent in the way projects are run in Brazil and Argentina though without serious implications in terms of knowledge sharing and transfer or project completion. Power distance could have possibly become a factor if the public sector would have taken an active role from the early stages of the Corridor with respect to not only the private sector stakeholders but also with intra-regional authorities. Aspects of superiority, dominance, rivalry, and possibly distrust amongst the regional agencies - although not yet experienced - 
might well be factors to consider upon a more active interaction between the private and public sectors at regional level within the roundtable forums.

Table 10.6: Response to Research Question 5

\begin{tabular}{|l|l|}
\hline Question 5 & $\begin{array}{l}\text { What factors hold a more relative influence on stakeholders to allow vertical } \\
\text { or horizontal integration to occur? }\end{array}$ \\
\hline Main Themes & $\begin{array}{l}\text { Change management, value chain, reappraisal, changed world view from } \\
\text { myopic to global through exposure to global players' practices. Leadership } \\
\text { style and influence. }\end{array}$ \\
\hline Comments & $\begin{array}{l}\text { The Corridor facilitated a change in leadership style due to the need for } \\
\text { parties to exchange knowledge and form alliances or joint ventures. The } \\
\text { industry structure and leadership style influenced impediments to changing } \\
\text { organizational structures to form joint ventures but the external shock } \\
\text { provided the necessary impetus (discussed in Chapter 2) to radically change } \\
\text { the value chain. }\end{array}$ \\
\hline
\end{tabular}

Section 3.2 discusses the industry structure and factors influencing organizational structure changes. Section 3.6 discussed business cooperation theory which is relevant to this research question along with the discussion of the region's context in Chapter 2.

The trust building process started as a natural consequence of participants having already identified the potential impact that globalization was going to have on the region and decided to find a way to reconfigure their businesses in some way or other but they had only a vague idea of how to do this. It becomes apparent from the representative case study examples of the many projects spawned by the Corridor that the main factors were given by the stakeholders' determination to reengineer their businesses to better face the new context. Such a process entailed an active process of knowledge sharing and transfer, cooperation, collaboration, and most importantly leadership. The latter turned out to be of paramount importance not only with respect to the leading attitude some companies adopted toward an integration strategy but also as a model to follow by many others holding a lesser market position and influence on other stakeholders. There was a strong relationship between a relatively small number of leading companies that took the initiative to reengineer and engage to various degrees of integration, and a greater number of followers. They were seduced both by the leaders' firm attitude and access to an open knowledge market that tended to undertake proactive behaviors in becoming integrated. Many lone runners became integrated to defend their market position or even expand their scope 
of services based on combined larger economies of scale, wider geographical scope of services, higher productivity, and enhanced knowledge, all within an innovative spirit that only the global threat seems to have put in place.

The case studies illustrate how the Corridor provided the $b a$ discussed earlier that allowed participants to explore how they could contribute to an integrated joint venture through their specialized input into a re-engineered value chain. This led to unexpected opportunities and forms of vertical and horizontal business integration.

Table 10.7: Response to Research Question 6

\begin{tabular}{|l|l|}
\hline Question 6 & $\begin{array}{l}\text { What is the actual awareness degree among stakeholders with respect to } \\
\text { sustainable development and its future relevance over the region? }\end{array}$ \\
\hline $\begin{array}{l}\text { Main } \\
\text { Themes }\end{array}$ & Change management, value chain, reappraisal, changed world view. \\
\hline Comments & $\begin{array}{l}\text { Initially before the Corridor facilitated a change in participants forming } \\
\text { alliances or joint ventures, the industry appeared complacent, parochial and } \\
\text { ignorant of technical advances in a range of logistics and business } \\
\text { technologies that posed the external shock that provided the necessary } \\
\text { impetus (discussed in detail in Chapter 2) to radically change the value chain. } \\
\text { What is remarkable about the evidence from the case studies presented is that } \\
\text { Corridor evolved as a peculiar and unique response by businesses operating in } \\
\text { a vast region of the world where local knowledge turned out to be a highly } \\
\text { valuable strategic asset. The resulting experience of reconfiguring business } \\
\text { may prove to be a valuable lesson learned with respect to the recent global } \\
\text { financial crisis. }\end{array}$ \\
\hline
\end{tabular}

Chapter 2 provides a detailed context to the region as well as its players. There was a remarkable degree of myopia in anticipating the impact of globalization upon this far flung region of the world economy, notwithstanding the fact that many of the participants in the case studies were highly business-literate individuals with high level qualifications that would have prepared them for understanding theories related to the nature of the firm, competitive advantage and the context of their region.

The case studies demonstrated: (1) how unprepared the region was to face the globalization impact; and (2) uncovered a false sense of security granted by protective laws and political 
regimes. These misconceptions were regarded by the companies as sustainable at that time. However, the Corridor project not only contributed to accomplish a major business transformation process but also to install in the minds and spirits of the regional logistics players that their only way towards sustainable business or to be globally competitive was to undertake a permanent business transformation. This entailed a constant change and innovation process facilitated through corporate networking, sharing and transferring knowledge, cooperation, collaboration, and leadership. The Corridor is regarded as a fundamental tool for the general betterment of the regional logistic sector as well as the most adequate entity to anticipate trends that help companies make their businesses sustainable. The situation is summarised using a PM perspective and illustrated in Figure 10.1.

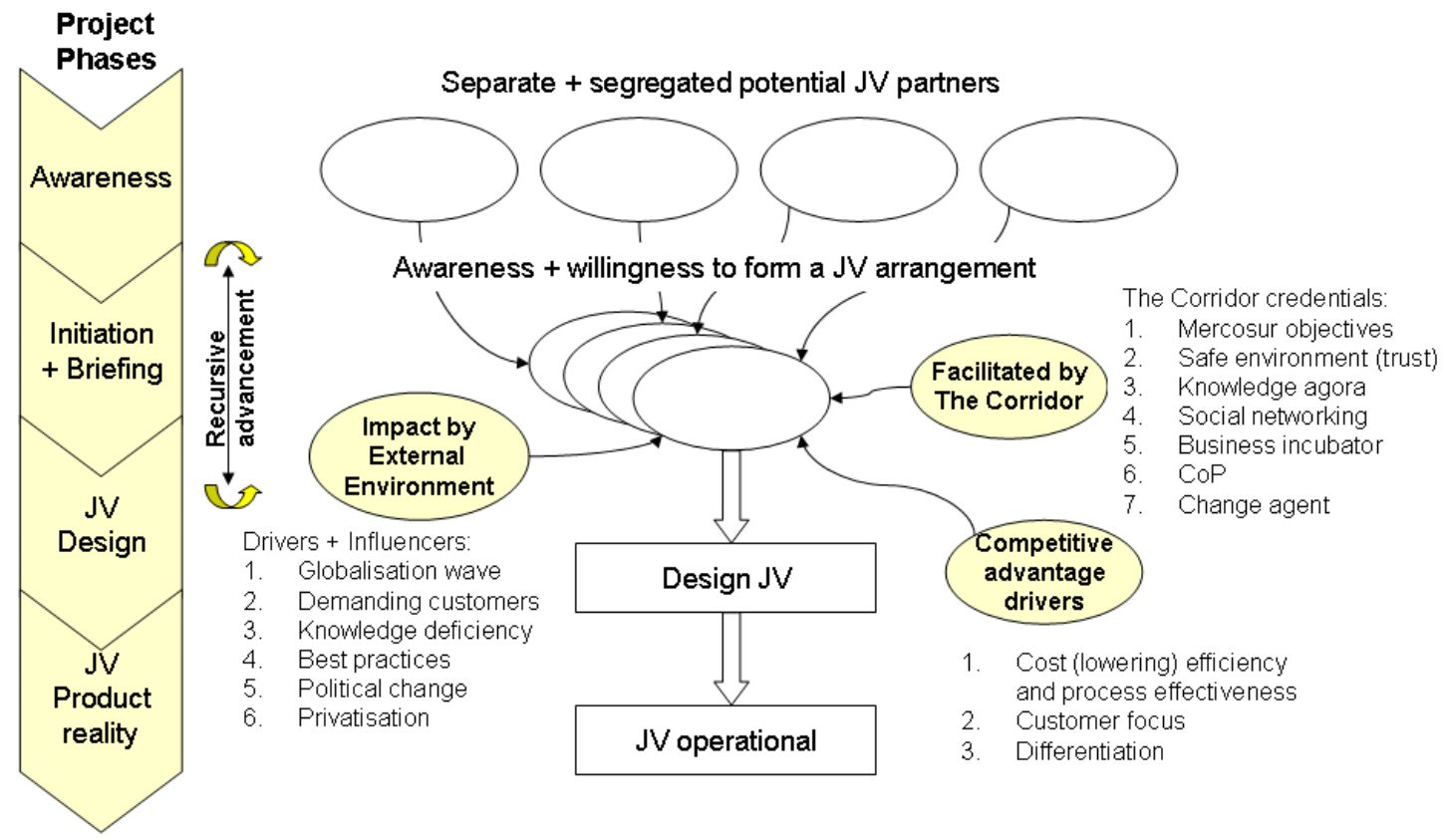

Figure 10.1: View of the Corridor's Role in JV Development

Figure 10.1 illustrates the formation of a JV through using a PM perspective based on the research findings. The Corridor project business transformation process passed through an awareness gestation phase then through to initiation and briefing where various forms, combinations of roles and ways of collaborating were discussed and decided upon. This was followed by the actual design of the JV and that passed into the reality of the JV. The thesis focuses upon the end of the awareness phase as it recursively slipped into the initiation and 
briefing phase and then into the start of the design of JVs and how they may be formed. Figure 10.1 also summarizes how the external environment impacted upon this process, how competitive advantage helped shape the drivers for change and how the Corridor facilitated the shaping of JVs and alliances.

At the initial stage, the separate parties joined together under the facilitation and auspices of the Corridor. This entity was sanctioned by advancing the MERCOSUR ideals and it was established as a safe (trustworthy) place in which representatives of member firms could meet and exchange knowledge and to solve problems. This helped the engaged firms to form a CoP and later more intimate groups coalesced into JV type arrangements and so the Corridor also had a role as a business incubator and change agent.

The role of the Corridor fulfils an important stakeholder engagement role. Stakeholders included the MERCOSUR member states and their constituencies, the firms that joined the Corridor as well as customers and clients of these firms. The Corridor, therefore, helped to draw together stakeholder representatives through the roundtable discussions. Stakeholder engagement is an important element of designing value into any project procurement arrangement (Walker, Bourne and Rowlinson, 2008). The project phases during which all this intense interaction took place was the awareness, initiation and recursive design and development of the JV.

\section{4- LIMITATIONS, RECOMMENDATIONS, AND FUTURE POTENTIAL}

This thesis research methodology was based on a qualitative approach where personal interviews and both my direct observation and participation in most of the cases were crucial to determine what the findings of the study turned out to be. A quantitative approach might have accurately identified some useful economic data relating to business failures, trends in changed company formation and changes in profits or losses sustained over the studied period. However, these can never explain what was actually happening on the ground, what was motivating and influencing such changes and how it all transpired the way it did. The research questions are framed around gaining an understanding about issues that could not effectively be studied using quantitative data. I had the opportunity as an active participant and reflective practitioner with strong connections to the players and a deep understanding of the 'soft' variables at play such as culture, 
geography, and business practice, to be able to gather qualitative data to help explain subtle issues at the micro level as well as my understanding of the Corridor's role at the macro level.

A quantitative approach might have been perhaps more accurate in establishing certain parameters or identifying some trends which otherwise may be influenced by my past professional experience, cultural origin, and personal views on certain topics. Even though this may pose a limitation on the depth of this thesis' findings, I have paid special attention to these aspects in order to avoid misinterpreting the findings.

The justification for using a qualitative rather than quantitative research design was the need to access deep and rich data and insights provided by participants and my own role as a reflective practitioner. This kind of research design makes replication impossible (as much of the data and insights are highly idiosyncratic) even though the approach could be mimicked for other similar research projects by others in a similar fortunate position to have similar access. In this sense it contributes and adds to insights about how a practitioner can develop reflective skills.

This thesis does not delve into the role or influence that the public sector might have played or exerted on the Corridor as an entity and all of the case studies, but that limitation does refer to this aspect as a pending issue subject to further study. The absence of this analysis raises a question mark as regards the interaction of the public and private sectors from a project management perspective as well as its potential impact on the business transformation trend the Corridor has so vigorously commenced.

This study did not address the IT component by which the many roundtable forums were integrated and their insights and results (tacit and explicit knowledge) circulated amongst the stakeholders. Doing this was beyond the scope of the DPM thesis that would have led to developing an entirely new thesis. Consequently this project opens up the possibility to deepen insights further from a communications technology perspective.

A clear limitation and therefore a potential recommendation for future study is the geopolitical perspective the Corridor holds with respect to its clearly dynamic dimension in terms of 
innovation and expansion. Innovation leads to cope with new ideas and concepts that can be added to the betterment of the various sectors and the region as a whole, and expansion entails adhering new countries and segments needing business transformation.

Further study is recommended on the comparative analysis of different regions of the world where structural asymmetries can be found in various regions where production and consuming poles can be brought together in far more efficient ways (e.g. Australia/New Zealand and south east Asia; southern Europe and North Africa; USA/Canada and central America \& the Caribbean; eastern Europe and the Middle East). Maritime affairs in the ground of fisheries, off-shore oil and gas, wind energy, sea-bed mining, marine environment, tourism and even Antarctic interests might find it useful to be integrated within a Corridor-like organization and to simultaneously tackle a number of supranational aspects of economic and geopolitical dimensions.

The Corridor's future potential is therefore defined by its capacity to generate not only defensive strategies to overcome a volatile and uncertain business context, but also to transform realities by means of cooperation, collaboration, culture, knowledge, and leadership put all together to work. The Corridor model may turn out to be useful anywhere structural asymmetries are found and trading practices need substantial improvement.

Finally, the recent global financial crisis has not been considered at all because the data gathering and validation phase of this thesis preceded that crisis. In many ways it bears similarities, as a massive shock, to the economic system in the way that the globalization wave triggered changes facilitated by the Corridor. It would be interesting for future research to be undertaken to track and better understand the way that the Corridor partners may be a) better prepared to cope with the consequences of this crisis and b) what form that coping and adjustment mechanism may take, particularly as government agencies may be a significant player in that process as appears to be the emerging case as governments move towards direct interventions.

\section{0-5 INSIGHTS AND SUGGESTED CORRIDOR IMPROVEMENTS}

The Corridor has been shown to be an unusual, even a unique, entity. This thesis has not proposed to assign a particular term that can describe beyond being a form of Strategic CoP as 
the title conveys. The Corridor is a commercial entity that was initially formed to facilitate business transformations, improvements in business processes and knowledge exchange to overcome common (to participants) challenges posed by the globalization wave and the implications of the MERCOSUR. However, its role grew more significant as a market for knowledge exchange and its role as brokering firm collaboration, supply chain integration, formation of radical business transformation through JVs and forms of alliance. It became a unique business incubator and model for managing complex programs of business change projects. While this may imply a role akin to a strategic PMO, the Corridor never had the authority to impose strategy and indeed there was not an intention to do so. While it was able to draw out from participants involved in its transformation projects in particular, sound practices that could be shared and adapted by other participants it was not an authority that could demand anything. It resembled a kind of midwife that delivers babies but it also (to use that analogy) was a matchmaker that brought firms together to conceive new possibilities. Knowledge and skill was the currency used in facilitating these transformations. Trust and commitment in members was inspired by the form of leadership adopted that fitted the culture.

In this regard, the Corridor is best viewed as a form of $\mathrm{CoP}$ but it takes a unique place in the continuum of CoPs from wholly anarchic ones based on disparate individuals with a common passion to a calculative corporative CoP that has been established by some form of recognized powerful authority that retains influence over its members.

The Corridor's strength is its tacit and collegial support that it has managed to gain so that commitment is generally affective rather than normative or continuance (Meyer and Allen, 1991). Two major improvements have been suggested in this thesis that the Corridor could consider. First, is that the possibility of government participation should be explored and enacted so long as the special authority of the Corridor through its trust is not compromised. Second, the IT supporting infrastructure that was for scope reasons excluded from the thesis could be reviewed as advances in this area have been radical over the past decade. 


\section{6- CHAPTER SUMMARY}

This chapter concludes the thesis. It has presented structural conclusions relevant to the thesis and also restated the research proposition and summarized the main factors that underpin it.

Responses to the six research questions posed was also summarized and explained using Figure 10.1 to presented a model of the formation of JVs through the Corridor's facilitation taken from a PM perspective. The research limitations were explained and further research gaps were identified for future researchers to address. The final section ended with a speculative comment relating to the emerging global financial crisis that gripped the world during the end of 2009 while this thesis was being written up and so is beyond the scope of further research. 


\section{REFERENCES}

Adler, L. (1979). “La Strategie de la Simbiose”, Harvard L’Expansion vol.14 pp58-98.

Adler, P. and Adler, P. (1994). “Observational techniques”, in N. Denzin and Y. Lincoln (eds) Handbook of Qualitative Research, Thousand Oaks CA: Sage, pp377-92.

Agencia Brasil (2000). Comunicado de Brasilia, na reuniao de presidentes da América do Sul. http://www.radiobras.gov.br/abr/integras/00/integra_0109_3.htm (October 16, 2000).

Alterini, A.A. \& Boldorini M.C.(1995). El Sistema Juridico en el MERCOSUR; 1, Estructura General - Instrumentos Fundacionales y Complementarios ; Abeledo-Perrot, Buenos Aires.

Anderson, J. C., Narus, J. A. and van Rossum, W. (2006). “Customer Value Propositions in Business Markets.” Harvard Business Review. 84 (3): 90-99.

Archer, N. P. and Ghasemzadeh, F. (1999). "An integrated Framework for Project Portfolio Selection.” International Journal of Project Management. 17 (4): 207-216.

Arranz Pena, N. \& Fernandez J.C. (2002). Business Cooperation: from theory to practice.

Palgrave MacMillan , New York.

Arroyo, A. C. and Walker, D. H. T. (2004). A Latin American Strategic Community of Practice Experience. 18th ANZAM Conference, Dunedin, New Zealand, 8-11 December, ANZAM: 11 pages on CD-Disk proceedings.

Arroyo, A. and Walker, D. H. T. (2008). Business Transformation Through an Innovative Alliance. In Procurement Systems - A Cross Industry Project Management Perspective. Walker D. H. T. and S. Rowlinson eds. Abingdon, Oxon, Taylor \& Francis: 423-444. Arroyo, A. C. and Walker, D. H. T. (2009). A Latin American Strategic Organisational Transformation Project Management Experience: The Motivation to Transform Business. Proceedings of the European Academy of Management EURAM: Renaissance \& Renewal in Management Studies, Liverpool UK, May 11-14 20pp pages on CD-Disk proceedings.

Artto, K. A. (2001). Management of project-orieted Organization - Conceptual Analysis. Project Portfolio Management - Strategic Management Through Projects. Karlos Artto M. M., \& Taru Aalto. Helsinki, Project Management Association of Finland: 5-22.

Artto, K. and Kujala, J. (2008). “Project Business as a Research Field.” International Journal of Managing Projects in Business. 1 (4): 469-497. 
Atkinson, J. W. (1957). “Motivational determinants of risk-taking behavior.” Psychological Review. 64 (6, Part 1): 359-372.

Auguste, B.G.; Hao, Y.; Singer, Y. \& Wiegand, M. (2002). The Other Side of Outsourcing; McKinsey Quarterly, 1, 52-63.

Bass , B.M. \& Avolio , B.J. (1994) . Improving organizational effectiveness through transformational leadership ; Sage, Thousand Oaks ; pp13-19 .

Bass , B.M. \& Stogdill , R.M. (1990) . Bass \& Stogdill's Handbook of Leadership ; Theory, Research , and Managerial Applications ; $3^{\text {rd }}$ edn ; The Free Press , New York ; pp383-86

Becker, H.S. (1958). Problems of inference and proof in participant observation. American Sociological Review, 23, 652-660.

Bennis , W.G. \& Nanus , B. (1985) . Leaders: The strategies for taking charge. New York : Harper \& Row .

Blomquist, T. and Müller, R. (2006). Middle Mangers in Program and Project Portfolio Management: Practices, Roles and Responsibilities. Newtown Square, PA Project Management Institute.

Boske , L.B. (2001) . Maritime Transportation in Latin America and the Caribbean. Lyndon B. Johnson School of Public Affairs . The University of Texas at Austin ; Policy Research Project Report \# 138 ; pp226-8 .

Brown, J.S. and Duguid, P. (2000). The social life of information. Harvard Business School Press.

Bryan , L.L. \& Hulme , R. (2003) . "Managing for improved corporate performance.” The McKinsey Quarterly, Number 3 .

Bryan, L. (2004). “Making a Market in Knowledge.” The McKinsey Quarterly. August 17: 1-6.

Bourne, L. and Walker, D. H. T. (2005). "Visualising and Mapping Stakeholder Influence.” Management Decision. 43 (5): 649-660.

Buchanan, N.S. (1940). The Economics of Corporate Enterprise. New York, Holt; p209.

Burton, S. \& Steane, P. (2004). Surviving Your Thesis. Routledge Taylor \& Francis Group London and New York;

Burton-Jones , A. (1999) . Knowledge Capitalism . Oxford, Oxford University Press.

Cappelli, P. (2000). “A Market-Driven Approach to Retaining Talent.” Harvard Business Review. 78 (1): 103-111. 
Cardoso, F.H. (2006). The Accidental President of Brazil, A Memoir; Public Affairs, New York; 173-7.

Cartwright, C. (2006). The journey to a "best in class" - Corporate Project Office. members P. M. C. Melbourne, PMI Melbourne Chapter.

Cartwright, C. and Walker, D. H. T. (2008). Case Study - Developing a Centre of Excellence (CoE). In Procurement Systems - A Cross Industry Project Management Perspective. Walker D. H. T. and S. Rowlinson. Eds, Abingdon, Oxon. Taylor \& Francis: Chapter 11. Christenson, D. (2007). Using Vision as a Critical Success Element in Project Management Doctor of Project Management, DPM, School of Property, Construction and Project Management. Melbourne, RMIT.

CIA World Factbook (2002). http://www.cia.gov/gov/cia/publications/factbook.htm

Cicimil, S. (2006). "Understanding Project Management Practice Through Interpretative and Critical Research Perspectives”. Project Management Journal, vol.37, No 2, 27-37.

Cicmil, S. and Hodgson, D.E. (2005). Knowledge, action, and reflection in management education - The case of project management. BBS Teaching and Research Review, Issue 7, September 2005.

Cleland, L. \& Ireland, L. (2002) . Project Management, strategic design and implementation . $4^{\text {th }}$ edn. ; McGraw Hill , New York ; pp455-64 ; 559-60.

Coffey, P. (1998). LATIN AMERICA-MERCOSUR; Kluwer Academic Publishers ; pp257-59.

Coghlan, D. (2001). “Insider Action Research Projects: Implications for Practising Managers.” Management Learning. 32 (1): 49-60.

Coghlan, D. and Brannick, T. (2005). Doing Action Research in Your Own Organization. Second. Edition, London, Sage Footprint.

Coghlan, D. and Brannick, T. (2007). Doing action research in your own organization. SAGE, London.

Cohen, D. and Prusak, L. (2001). In good company : how social capital makes organizations work. Boston Harvard Business School Press.

Cohen, R. \& McCluskey, I. (1996). Keep it in the Neighborhood Forget NAFTA - South America is busy Building its Own Powerful trading Bloc, Called MERCOSUR; Time Magazine , August 26 $6^{\text {th }}, 1996$.

Cohen, W.M. \& Levinthal, D.A. (1990). “Absorptive Capacity: A New Perspective on Learning and Innovation,” Administrative Science Quarterly 35: PP128-152. 
Collins, T.M. \& Doorley, T.M. (1992). Les Alliances Strategiques (Paris:Inter- Editions)

Conner, K. R. and Prahalad, C. K. (1996). “A Resource-based Theory of the Firm: Knowledge Versus Opportunism.” Organization Science: A Journal of the Institute of Management Sciences. 7 (5): 477-501.

Cooke-Davies, T. (2002). “The "Real" Success Factors on Projects.” International Journal of Project Management. 20 (3): 185-190.

Courtney, H., Kirkland, J. and Viguerie, P. (1997). “Strategy Under Uncertainty.” Harvard Business Review. 75 (6): 67-81.

Crawford, J. K. (2002). The Strategic Project Office.New York, USA Marcel Dekker AG.

Crawford, L. (2006). Project Governance - The Role and Capabilities of the Executive Sponsor. PMOZ - Achieving Excellence, Melbourne, Australia, 8-11 August, PMI -Melbourne Chapter: 1-11 CD-ROM paper.

Creswell, J.W. (1998). Qualitative Inquiry and Research Design: Choosing Among Five Traditions, thousand Oaks CA: Sage.

Cyert, M. \& March, J.G. (2006). A Behavioral Theory of the Firm. Blackwell Publishing; Massachussets.

Davenport, T.H. \& Prusak, L. (2000) . Working Knowledge. How organizations manage what they know. Harvard Business School Press Boston; pp93-106 .

Davenport, T.H. (1993). Process Innovation: Reengineering Work through Information Technology. Harvard Business School Press, Boston, Massachussets.

David, F.R. (2003) . Strategic Management Concepts; Prentice Hall , New Jersey , $9^{\text {th }}$ edition; pp157-185.

Davidow, W.H. \& Malone, M.S. (1992). The Virtual Corporation: Structuring and Revitalizing the Corporation for the $21^{\text {st }}$ Century. Harper-Collins, New York.

Davidson, K. (1987). “Do Megamergers Make Sense?” Journal of Business Strategy 7 , no 3:45.

Dawson, P. (1997). "In at the deep end: conducting processual research on organizational change”. Scandinavian Journal of Management, 13 (4): 389-405.

De Jonquiers, G. (2001). "Popular Trend is at Odds with Global Free Trade: Regional trade Agreements"; The Financial Times, London; November 30 ${ }^{\text {th }}, 2001$. 
Denzin, N.K. and Lincoln, Y.S. (2000). “The discipline and practice of qualitative research", in N.K. Denzin and Y.S. Lincoln (eds.) Handbook of Qualitative Research, $2^{\text {nd }}$ edn., Thousand Oaks CA: Sage, pp. 1-28.

Dixon, N. M. (2000). Common Knowledge. How Companies Thrive by Sharing What They Know. Harvard Business School Press, Boston, Mass.

Donaldson, T. and Preston, L. E. (1995). “The Stakeholder Theory of the Corporation: Concepts, Evidence, and Implications.” Academy of Management Review. 20 (1): 65-91.

Doz, Y. L. and Hamel, G. (1998). Alliance Advantage - The Art of Creating Value Through Partnering. Boston, Harvard Business School Press.

Drucker, P. F. (1999). “Knowledge-Worker Productivity: The Biggest Challange.” California Management Review. 41 (2): 79-94.

Durell, P. (2005). Arising Tide Lifts all Boats ? TMF Admiral. Economic Commission for Latin America and the Caribbean (1988). "The Impact of Structural Changes in Liner Shipping on Caribbean Ports”, FAL Bulletin, 142 (April 1988).

Eisenhardt, K. M. and Brown, S. L. (1999). "Patching Restitching Business Portfolios in Dynamic Markets.” Harvard Business Review. 77 (3): 73-82.

Eisenhardt, K. M. and Martin, J. A. (2000). “Dynamic Capabilities: What are They?” Strategic Management Journal. 21 (10/11): 1105-1121.

Elashmawi, F. \& Harris, P.H. (1998). Multicultural Management 2000 : essential cultural insights for global business success. Gulf Publishing , Houston, Texas. p87

Elkington, J. (1997). Cannibals with Forks.London Capstone Publishing.

Evans, D. (1995). How to Write a Better Thesis or Report. Melbourne: Melbourne University Press.

Ferdows, K. (1997). “Making the Most of Foreign Factories.” Harvard Business Review. 75 (2): 73-88.

Fernie, S., Green, S. D., Weller, S. J. and Newcombe, R. (2003). "Knowledge Sharing: Context, Confusion and Controversy.” International Journal of Project Management. 21 (3): 177187.

Ferreira, L.D. and Merchant, K.A. (1992) "Field Research in management accounting and control: a review and evaluation”, Accounting, Auditing and Accountability Journal, 5 (4): 3-34.

Flick, U. (2002). An Introduction to Qualitative Research, London: Sage. 
Flyvbjerg, B. (2001). Making social science matter: Why social inquiry fails and how it can succeed again. Cambridge: Cambridge University Press.

Frankel, E.G. (1980). Regulation and policies of American Shipping. Massachusetts Institute of Technology; Ausburn Publishing, Boston ; pp130-140.

Garvin, D.A. (1998). “Building a Learning Organization”. Harvard Business Review on Knowledge Management. Harvard Business School Press, Boston, MA; pp48-78.

Ghoshal. S. (1996). “Theories of Economic Organization: The Case for Realism and Balance”. Academy of Management Review.21 (1):58-73.

Gibson, K. (2000). “The Moral Basis of Stakeholder Theory.” Journal of Business Ethics. 26: 245-257.

Gladwell, M. (2000). The Tipping Point.London Abacus.

Glaser, B.J. and Strauss, A.L. (1967). The Discovery of Grounded Theory: Strategies for Qualitative Research. New York Aldine Pub. Co.

Glesne, C. (1999). Becoming Qualitative Researchers: An Introduction, $2^{\text {nd }}$ edn, New York: Longman.

Graduate School of Business, RMIT 2002 - Welcome to the Doctor of Project Management ; Information Package ; pp2-3

Greiner, L. E. (1998). “Evolution and Revolution as Organizations Grow.” Harvard Business Review. 76 (3): 55-68.

Greiner, L. E. and Schein, V. (1988). Power and Organisation Development. New York Addison-Wesley Management.

Gubrium, J.F. and Holstein, J.A. (2000). “Analyzing Interpretive Practice“, in N.K. Denzin and Y.S. Lincoln (eds) Handbook of Qualitative Research, 2nd edn, Thousand Oaks CA: Sage, pp. 487-508.

Gupta, V., Hanges, P. J. and Dorfman, P. (2002). “Cultural Clusters: Methodology and Findings.” Journal of World Business. 37 (1): 11-15.

Hamel, G. and Prahalad, C. K. (1994). Competing for the Future. Boston Harvard Business School Press.

Handler, T. J. and Magee, F.(1999). Multiple Styles for Implementing a Project Office, Gartner Group,: 4.

Handy, C. (1992). The Age of Unreason. Harvard Business School Press, Boston, Massachussets. 
Hansen, M. T., Nohria, N. and Tierney, T. (1999). "What's Your Strategy for Managing Knowledge?” Harvard Business Review. 77 (2): 106-116.

Haralambides, H.E. (2000). “A Second Scenario on the Future of the Hub-and-Spoke System in Liner Shipping”; paper presented to the Latin ports \& Shipping 2000 Conference \& Exhibition, Miami, FL.

Herriott, R.E. and Firestone, W.A. (1983). Multisite qualitative policy research: Optimizing description and generalizability. Educational Researcher, 12, 14-19.

Hersey, P. \& Blanchard, K. (1982). Management of Organizational Behaviors. Englewood Cliffs.; NY.

Hersey, P.; Blanchard, K. \& Johnson, D.E. (1996). Management of Organizational Behavior: Utilizing Human Resources ( $7^{\text {th }}$ ed.) Upper Saddle River, NJ, Prentice Hall.

Hobbs, B. and Aubry, M. (2007). “A Multi-Phase Reserach Prram Investigating Project Management Offices (PMOs): The Results of Phase 1.” Project Management Journal. 38 (1): 74-86.

Hodgson, D. E. (2004). "Project Work: The Legacy of Bureaucratic Control in the PostBureaucratic Organization.” Organization. 11 (1): 81-100.

Hodgson, D. and Cicmil, S. (2006a). Making Projects Critical.Basingstoke, UK Palgrave MacMillan.

Hodgson, D. and Cicmil, S. (2006b). Are projects real? The PMBOK and the legitimation of project management knowledge. Making Projects Critical. Hodgson D. and S. Cicmil. Ed. Basingstoke, UK. Palgrave MacMillan: 29-50.

Hoecklin, L. A. (1995). Managing cultural differences : strategies for competitive advantage.Wokingham, England ; Reading, Mass. Addison-Wesley.

Hoffmann, J. (1998)." Concentration in Liner Shipping: Its Causes and Impacts for Ports and Shipping Services in Developing Regions”. Economic Commission for Latin America and the Caribbean. Santiago, Chile; p12.

Hofstede, G. (1991). Culture and Organizations: Software of the Mind.New York McGraw-Hill. Hofstede, G. H. (1980). Culture's Consequences: International Differences in Work-Related Values.Beverly Hills Sage.

Hofstede, G. (1991). Culture and Organizations: Software of the Mind.New York McGraw-Hill. House, R., Javidan, M., Hanges, P. and Dorfman, P. (2002). "Understanding Cultures and Implicit Leadership Theories Across the Globe: An Introduction to Project GLOBE.” Journal of World Business. 37 (1): 3-10. 
Ingebretsen, M. (2003). “Win Project Battles Managers Enlist Project War Rooms.” Journal.: 2630

International Agreements: Argentina’s Menem to propose single currency for MERCOSUR”; 15th International Trade Reporter 422; March 11 ${ }^{\text {th }}, 1998$.

International Agreements: US exports to MERCOSUR members tripled in 1990-1996, ITC paper says” 15th International Trade Reporter 1432, August 19, 1998 “International Agreements: MERCOSUR Trade Volume Seen Rebounding in 2000,” ; 17th International Trade Reporter 1454 ; September $21^{\text {st }}$, 2000.

James, B.G. (1985). “Alliance : The New Strategic Focus”; Long Range Planning, vol.18,3; pp76-81.

Jesuino, J. C. (2002). “Latin Europe Cluster: From South to North.” Journal of World Business. 37 (1): 81-89.

Jewell , M. and Walker , D.H.T. (2004) . Community of Practice Perspective Software Management Tools : A UK Construction Company Case Study . Knowledge Management in the Construction Industry : A Socio-Technical Perspective . Kazi A. S.

Jones, T. M. and Wicks, A. C. (1999). “Convergent Stakeholder Theory.” Academy of Management Review. 24 (2): 206-221.

Kim, W. C. and Mauborgne, R. (2003). “Tipping Point Leadership.” Harvard Business Review. 81 (4): 60-70.

Kogut, B. (1988). “Joint Ventures: Theoretical and Empirical Perspectives”, Strategic Management Journal, vol.9, pp319-32.

Kotter , J.P. (1998) . "What Leaders Really Do” . Harvard Business Review on Leadership . Harvard Business School Publishing, Boston ; p40 .

Kotter, J. P. (1995). “Leading change - Why Transformation Efforts Fail.” Harvard Business Review. 73 (2): 59-67.

Krass, P. (2001). “Why Do We Do It?” Across the Board (May-June 2001):23.

Larkin , T.J. \& Larkin , S. (1999) . "Reaching and Changing Frontline Employees” ; Harvard Business Review on Effective Communication ; Harvard Business Scholl Publishing , Boston ; p152 .

Lave, J. and Wenger, E. C. (1991). Situated Learning - Legitimate Peripheral Participation.Cambridge Cambridge University Press. 
Lewin, K. (1951). Field Theory in Social Science: Selected Theoretical Papers. New York: Harper \& Row.

Livingston , J.S. (2000) . "Pygmalion in Management”. Harvard Business Review on Managing People; Harvard Business School Publishing , Boston ; pp55-57 .

Livingston, J. S. (1969). “Pygmalion in management.” Harvard Business Review. 47 (4): 81-89.

Llewellyn, S. (1993). "Working in hermeneutic circles in management and accounting research: some implications and applications”, Management Accounting Research, 4:231-49

Lucey, T. (1997). Management Information Systems; Continuum, London; Chapter 2.

Malfroy, J. (2004). Conceptualisation of a Professional Doctorate Program: Focusing on Practice and Change ; The Australian Educational Researcher Volume 31 Number 2; pp68-70.

Markides. C.C.(1998). “To Diversify or Not to Diversify”. Harvard Business Review on Strategies for Growth. Harvard Business School Publishing, Boston; pp79-96.

Markus, L.M. (2001). “Toward a Theory of Knowledge Reuse: Types of knowledge reuse situations and Factors in Reuse Success..”.Journal of Management Information Systems; Vol.18, Nbr.1; pp57-93.

Mayer, R. C., Davis, J. H. and Schoorman, F. D. (1995). “An Integrated Model of Organizational Trust.” Academy of Management Review. 20 (3): 709-735

McDermott, R. (1999). "Why Knowledge Technology Inspired but Cannot Deliver Knowledge Management.” California Management Review. 41 (4): 103-117.

McNiff, J. and Whitehead, J. (2000). Action Research in Organizations. London Routledge.

Meyer, J. P. and Allen, N. J. (1991). “A Three-Component Conceptualization of Organizational Commitment.” Human Resource Management Review. 1 (1): 61-89.

Miles, M.B. and Huberman, A.M. (1994). An Expanded Sourcebook: Qualitative Data Analysis, $2^{\text {nd }}$ edn, Thousand Oaks CA: Sage.

Mintzberg, H. (1987). “Crafting Strategy.” Harvard Business Review. 65 (4).

Mintzberg, H., Ahlstrand, B. W. and Lampel, J. (1998). Strategy Safari : The Complete Guide Through the Wilds of Strategic Management. London; Financial Times/Prentice Hall. Mullin , R. (1996) . "Knowledge Management : A Cultural Evolution” . Journal of Business Strategy, September-October , 56-59 .

Muto, S. (2001). “Seeing a Boost, Hospitals Turn to Retail Stores”, Wall Street Journal (November 7, 2001):B1 \& B8.

Nahapiet, J. and Ghoshal, S. (1998). "Social Capital, Intellectual Capital, and the Organizational Advantage.” Academy of Management Review. 23 (2): 242-266. 
Neumann, W.L. (2003). Social Research Methods: Qualitative and Quantitative Approaches, $5^{\text {th }}$ edn, Boston MA: Pearson.

Nichols , R.G. \& Stevens , L.A. (1999) . "Listening to People” . Harvard Business Review on Effective Communication . Harvard Business School Publishing, Boston ; pp8-14 .

Nonaka, I. (1991). The Knowledge-Creating Company. Harvard Business Review, Boston; Harvard Business School.

Nonaka, I. (1998). “The Knowledge Creating Company”. Harvard Business Review on Knowledge Management; Harvard Business School Publishing, Boston, MA. pp21-45.

Nonaka, I. and Konno, N. (1998). “The Concept of 'Ba': Building a Foundation for Knowledge Creation.” California Management Review. 40 (3): 40.

Nonaka, I. \& Takeuchi, H. (1995). The Knowledge-Creating Company: How Japanese Companies Create the Dynamics of Innovation. Oxford University Press, New York.

Nonaka, I. \& Teece D.J. (2001). Managing Industrial Knowledge: Creation, transfer and Utilization; SAGE, London.

Nonaka, I., Toyama, R. and Konno, N. (2001). SECI, Ba and Leadership: A Unified Model of Dynamic Knowledge Creation. Managing Industrial Knowledge - creation, transfer and utilization. Nonaka I. and D. Teece. Ed. London. Sage: 13-43.

Norrie, J. L. (2006). Improving Results of Project Portfolio Management in the Public Sector Using a Balanced Scorecard Approach. Doctor of Project Management, School of Property, Construction and Project Management. Melbourne, RMIT University.

Oliver, R.L. (1997). Satisfaction: A Behavioral Perspective on the Consumer. Boston MA: Irwin McGraw-Hill.

O'Neill, G. (2006). "Book Reviews - Pay without performance: The unfilled promise of executive compensation; The false promise of pay for performance: Embracing a positive model of the company executive " Asia Pacific Journal of Human Resources. 44 (3): 374-380.

O'Neill, G. and Berry, A. (2002). "Remuneration of Australian Executives: A Practitioner Review." Asia Pacific Journal of Human Resources. 40 (2): 228-245.

Oum, T. H., Park, J.-H. and Zhang, A. (2000). Globalization and Strategic Alliances - The Case of the Airline Industry.Oxford Pergammon.

Penrose, E. (1995). The Theory of the Growth of the Firm. Oxford University Press ; Oxford.

Pettigrew, A. M. (1997). “What is a processual analysis?” Scandinavian Journal of Management. 13 (4): 337-348. 
Pinfold, J. F. (2001). "The Expectations of New Business Founders: The New Zealand Case." Journal of Small Business Management. 39 (3): 279-285

PMI (2003). Organizational Project Management Maturity Model (OPM3) Knowledge Foundation.Newtown Square, Pennsylvania PMI.

PMI (2004), A Guide to the Project Management Body of Knowledge (PMBOK Guide); Project Management Institute, Third Edition; Four Campus Blvd, PA.

PMI (2006a). The Standard for Portfolio Management.Newtown Square, PA Project Management Institute.

PMI (2006b). The Standard for Program Management.Newtown Square, PA Project Management Institute.

Porter, M. E. (2001). “Strategy and the Internet.” Harvard Business Review. 79 (3): 63-78.

Porter, M.E ; Mariti, P.; et Smiley, R.H. (1983). “Cooperative Agreements and the Organization of Industry”. The Journal of Industrial Economics., vol.31, 4, pp437-51.

Porter, M.E. (1980). Competitive Strategy. Techniques for analizing industry and competitors. Free Press; London.

Porter, M.E. (1985). Competitive Advantage ; creating and sustaining superior performance. Free Press , London.

Porter, M.E. (1989). The Competitive Advantage of Nations. Free Press; London.

Prahalad, C. K. and Hamel, G. (1990). “The Core Competence of the Corporation.” Harvard Business Review. 68 (3): 79-91.

Rad, P. (2001). “Is Your Organization a Candidate for Project Management Office (PMO)?” AACE International Transactions. PM.07.01-04.

Rad, P. F. and Levin, G. (2002). The Advanced Project Management Office: A Comprehensive Look at Function and Implementation.Boca Raton, Florida St Lucie Press imprint of CRC Press.

Raelin, J. A. (2007). “Toward an Epistemology of Practice.” Academy of Management Learning \& Education. 6 (4): 495-519

Reason, P. \& Bradbury, H. (2001). Handbook of Action Research. Participative Inquiry \& Practice; Sage London; p38.

Sadler, I. (2007). Logistics and Supply Chain Integration.Thousand Oaks, CA Sage.

Scapens, R.W. (1990). "Researching management accounting practice: the role of case study methods”; British Accounting Review, 22 (3): 259-81. 
Schein, E. H. (1993). "How can Organisations Learn Faster? Lessons from the Green Room.” Sloan Management Review. 34 (2): 85-92.

Schlier, W.; Hunter, R.; Harris, K. \& Berg, T. (1998). Enterprise 2003: The Technology-Enabled Enterprise; Gartner Research; pp6-7.

Schon, D. (1983). The reflective practitioner: How professionals think in action. New York: Basic Books.

Senge, P. M. (1990). The Fifth Discipline - The Art \& Practice of the Learning Organization.Sydney, Australia Random House.

Sense, A. J. (2007). "Structuring the project environment for learning.” International Journal of Project Management. 25 (4): 405-412.

Sense, A. J. (2008). "Conceptions of learning and managing the flow of knowledge in the projectbased environment.” International Journal of Managing Projects in Business. 1 (1): 3348.

Shank, G. (2002). Qualitative Research: A Personal Skills Approach, Upper Saddle River NJ: Pearson.

Sherer, S. A. (2005). "From supply-chain management to value network advocacy: implications for e-supply chains.” Supply Chain Management. 10 (2): 77-83.

Sieber, S.D. (1973). The integration of fieldwork and strategy methods. American Journal of Sociology, 78, pp1335-1359.

Silverman, D. (1985). Qualitative Methodology and Sociology: describing the Social World, Aldershot: Gower.

Silverman, D. (2000). Doing Qualitative Research: A Practical Handbook, London: Sage.

Skyrme, D.J. (1999) . Knowledge Networking: Creating the Collaborative Enterprise ; Butterworth-Heinemann, Oxford ; pp.52-59 .

Smith, N.L. (1990). Cautions on the use of investigative case studies in meta-evaluation. Evaluation and Program Planning, 13(4), 373-78.

Spender, J.-C. (1996). "Making Knowledge the Basis of a Dynamic Theory of the Firm.” Strategic Management Journal. 17 (Winter Issue): 45-62.

Stake, R.E. (1986). Quieting reform: Social science and social action in an urban youth program. Urbana: University of Illinois Press.

Storck, J. and Hill, P. A. (2000). “Knowledge Diffusion Through "Strategic Communities".” Sloan Management Review. 41 (2): 63-74. 
Strauss, A. and Corbin, J. (1998). Basics of Qualitative Reserach.2nd.Thousand Oaks, CA. SAGE Publications Inc.

Styhre, A. (2006). “The bureaucratization of the project manager function: The case of the construction industry.” International Journal of Project Management. 24 (3): 271-276.

Sveiby , K.E. 2003-11-24 . www.sveiby.com

Szulanski , G. (2003) . Sticky Knowledge Barriers to knowing in the Firm . Thousand Oaks , CA ; Sage Publications .

Szulanski, G. (1996). "Exploring internal stickiness: Impediments to the transfer of best practice within the firm.” Strategic Management Journal. 17 (Winter special Issue): 27-43.

Szulanski, G. (1996). "Unpacking Stickiness: An Empirical Investigation of the Barriers to Transfer Best Practice Inside the Firm,” Academy of Management Journal 17 : pp437-441.

Szulanski, G. and Jensen, R. J. (2004). “Overcoming Stickiness: An Empirical Investigation of the Role of the Template in the Replication of Organizational Routines.” Managerial and Decision Economics. 25 (6-7): 347-363.

Tannenbaum, R. \& Schmidt, W.H. (1958). How to Choose a Leadership Pattern; Harvard Business Review, 46 (4), 95-101.

Teece, D., Pisano, G. and Shuen, A. (1997). "Dynamic Capabilities and Strategic Management.” Strategic Management Journal. 18 (7): 509-533.

Turner, J. R. (1999). The Handbook of Project-based Management: Improving the Processes for Achieving Strategic Objectives.2nd.London, UK McGraw-Hill.

Van Klink, H.A. (1997). “Creating Port Networks: the case of Rotterdam and the Baltic Region” ; International Journal of Transport Economics, vol24 , \#3, Oct 1997.-

Vivacqua, P.A. \& Stehling, S.M.F. A Logistic Project for South America’s Integration. Paper presented at OCDE Seminar Intermodal and Logistics Transportation Networks. Mexico City , June 1997.

Walker , D.H.T. ; Maqsood , T. and Finegan , A. (2004). The Culture of the Knowledge Advantage (K-Adv)-An Holistic Strategic Approach to the Management of Knowledge . Knowledge Management in the Construction Industry: A Socio Technical Perspective. Kazi , A.S. Helsinski , Finland , Idea Group Publishing , pp3-8. 
Walker, D. H. T. (2004). The Knowledge Advantage (K-Adv) Unleashing Creativity and Innovation, Unpublished report draft manuscript. Melbourne: 183.

Walker, D. H. T. and Christenson, D. (2005). “Knowledge Wisdom and Networks: A Project Management Centre of Excellence Example.” The Learning Organization. 12 (3): 275-291.

Walker, D. H. T. and Hampson, K. D. (2003). Enterprise Networks, Partnering and Alliancing. Procurement Strategies: A Relationship Based Approach. In Walker D. H. T. and K. D. Hampson Ed. Procurement a Relationship-based Approach, Oxford, Blackwell Publishing: Chapter 3, 30-73.

Walker, D. H. T., Arlt, M. and Norrie, J. (2008a). The Role of Business Strategy in PM Procurement. In Procurement Systems - A Cross Industry Project Management Perspective. Walker D. H. T. and S. Rowlinson Ed London, Taylor \& Francis: Chapter 5.

Walker, D. H. T., Bourne, L. and Rowlinson, S. (2008b). Stakeholders and the Supply Chain. In Procurement Systems - A Cross Industry Project Management Perspective. Walker D. H. T. and S. Rowlinson Ed. Abingdon, Oxon, Taylor \& Francis: 70-100.

Walker, D. H. T., Wilson, A. J. and Srikanathan, G. (2004). The Knowledge Advantage (K-Adv) For Unleashing Creativity \& Innovation in the Construction Industry. Brisbane CRC in Construction Innovation http://www.construction-innovation.info/images/pdfs/2001-004A_Industry_Booklet.pdf

Walker, R. (1985). Applied Qualitative Research, Aldershot: Gower.

Weick, K. E., Sutcliffe, K. M. and Obstfeld, D. (2005). "Organizing and the Process of Sensemaking.” Organization Science. 16 (4): 409-421.

Wenger, E. \& Snyder, W. (2000). Learning Communities, Workshop 5, KEFair 98. http://www.tmn.com

Wenger, E; McDermott, R. \& Snyder W.M. (2002). Cultivating Communities of Practice. Harvard Business School Press, Boston, Massachusetts.

Werner, O. and Schoepfle, G.M. (1987). Systematic Fieldwork: Foundations of Ethnography and Interviewing, Newbury Park CA: Sage, vols 1 and 2.

Williams, T. (2002). Modelling Complex Projects. London: Wiley.

Williamson, O.E. (1985). The Economic Institutions of Capitalism. The Free Press, NY.

Winter, M. and Checkland, P. (2004). Management and managing: Making sense of managing in organizations. 
Winter, M. and Gale, A. (2003). Developing a new kind of dissertation for a flexible professional development program. Manchester Centre for Civil and Construction Engineering, UMIST, UK.

Winter, M. and Thomas, J. (2007). Understanding the Lived Experience of Managing Projects: The Need for More Emphasis on the Practice of Managing. Manchester Centre for Civil and Construction Engineering, UMIST, UK.

Winter, M., Smith, C., Morris, P. W. G. and Cicmil, S. (2006). "Directions for future research in project management: The main findings of a UK government-funded research network.” International Journal of Project Management. 24 (8): 638-649.

Yin, R.K. (1981a). The case study as a serious research strategy. Knowledge: Creation, Diffusion, Utilization, 3, 97-114.

Yin, R.K. (1981b). The case study crisis: Some answers. Administrative Science Quarterly, 26, 58-65.

Yin, R.K. (1994). Case Study Research Design and Methods. Sage London.

Yukl , G. (2002) . Leadership in Organizations ; Upper Saddle River , New Jersey , $5^{\text {th }}$ edn. ; pp5-6 ; 348-352 ; 386-388

Zack, M.H. (1999). Knowledge and strategy (Knowledge Reader). Butterworth-Heinemann, USA.

Zaleznik , A. (1998) . “Managers and Leaders : Are they Different ?” . Harvard Business Review on Leadership . Harvard Business School Publishing ; Boston ; p62 . 


\section{GLOSSARY}

ALADI: Latin American Association of Economic Integration

ALL: America Latina Logistica

ATZ: Auto Terminal Zarate

BAP: Buenos Aires al Pacifico

CAFTA: Central America Free Trade Agreement

CARICOM: Caribbean Community

CET: Common External Tariff

CDS: Construction Differential Subsidy

COE: Centre of Excellence

COP: Communities of Practice

DPM: Doctor of Project Management

ECLAC: Economic Commission for Latin America and the Caribbean

ELMA: Empresa de Lineas Maritimas Argentinas

FO: Fuel Oil

GATT: General Agreement on trade and Tariffs

GPS: Global Positioning System

HCO: Health Care Organization

KM: Knowledge Management

KPI: Key Performance Indicator

MERCOSUR: Southern Common Market

MTC: Mercosur Trade Centre

NAFTA: North America Free Trade Agreement

NMC: Northern Maritime Corridor

NVOCC: Non-Vessel Operating Common Carrier

ODS: Operating Differential Subsidy

PMBOK: Project Management Body of Knowledge

PMO: Project Management Office

PNW: Pacific North West

RBV: Resource Based View 
RO/RO: Roll-on / Roll-ff

SAMEC or SAEC: South America East Coast

SAMWC or SAWC: South America West Coast

SECI: Socialization, Externalization, Combination, Internalization

3PL: Third Party Logistics

TCE: Transaction Cost Economics

TMM: Transportadora Maritima Mexicana

TAT: Turn-Around-Time

TEU: Twenty Equivalent Units

USEC+G: United States East Coast and Gulf

USEWC: United States West Coast

VSA: Vessel Sharing Agreement 


\section{APPENDICES}

\section{APPENDIX A: EXTRACT FROM THE TREATY OF ASUNCION}

\section{Treaty of Asunción 30 I.L.M. 1041 (March 26, 1991)}

Appendix I: Treaty of Asunción.

SOUTHERN COMMON MARKET (MERCOSUR) AGREEMENT (Original: Spanish)

Treaty Establishing a Common Market between the Argentine Republic, the Federal Republic of Brazil, the Republic of Paraguay and the Eastern Republic of Uruguay.

The Argentine Republic, the Federative Republic of Brazil, the Republic of Paraguay and the Eastern Republic of Uruguay, hereinafter referred to as the "States Parties'

CONSIDERING that the expansion of their domestic markets, through integration, is a vital prerequisite for accelerating their processes of economic development with social justice, BELIEVING that this objective must be achieved by making optimum use of available resources, preserving the environment, improving physical links, coordinating macroeconomic policies and ensuring complementarity between the different sectors of the economy, based on the principles of gradualism, flexibility and balance,

BEARING IN MIND international trends, particularly the integration of large economic areas and the importance of securing their countries a proper place in the international economy.

BELIEVING that this integration process is an appropriate response to such trends,

AWARE that this Treaty must be viewed as a further step in efforts gradually to bring about Latin American integration, in keeping with the objectives of the Montevideo Treaty in 1980,

CONVINCED of the need to promote the scientific and technological development of the States Parties and to modernize their economies in order to expand the supply and improve the quality of available goods and services, with a view to enhancing the living conditions of their populations, 165

Appendix I 167

166 Appendix I

REAFFIRMING their political will to lay the bases for increasingly close ties between their peoples, with a view to achieving the above-mentioned objectives, HEREBY AGREE AS FOLLOWS:

\section{CHAPTER I: PURPOSES, PRINCIPLES AND INSTRUMENTS}

\section{Article I}

The States Parties hereby decide to establish a common market, which shall be in place by 31 December 1994 and shall be called the "common market of the southern cone” (MERCOSUR). This common market shall involve:

The free movement of goods, services and factors of production between countries through, inter alia, the elimination of customs duties and non-tariff restrictions on the movement of goods, and any other equivalent measures;

The establishment of a common external tariff and the adoption of a common trade policy in relation to third States or groups of States, and the co-ordination of positions in regional and international economic and commercial forums;

The co-ordination of macroeconomic and sectoral policies between the States Parties in the areas of foreign trade, agriculture, industry, fiscal and monetary matters, foreign exchange and capital, services, customs, transport and communications and any other areas that may be agreed upon, in order to ensure proper competition between the States Parties;

The commitment by States Parties to harmonize their legislation in the relevant areas in order to strengthen the integration process.

Article 2

The common market shall be based on reciprocity of rights and obligations between the States Parties.

Article 3 
During the transition period, which shall last from the entry into force of this Treaty until 31 December 1994, and in order to facilitate the formation of the common market, the States Parties shall adopt general rules of origin, a system for the settlement of disputes and safeguard clauses, as contained in Annexes 11. III and IV respectively to this Treaty.

Article 4

The States Parties shall ensure equitable trade terms in their relations with third countries. To that end, they shall apply their domestic legislation to restrict imports whose prices are influenced by subsidies, dumping or any other unfair practice. At the same time, States Parties shall co-ordinate their respective domestic policies with a view to drafting common rules for trade competition.

Article 5

During the transition period, the main instruments for putting in place the common market shall be: (a) A trade liberalization programme, which shall consist of progressive linear and automatic tariff reductions accompanied by the elimination of non-tariff restrictions or equivalent measures, as well as any other restrictions on trade between the States Parties, with a view to arriving at a zero tariff and no non-tariff restrictions for the entire tariff area by 31 December 1994 (Annex I);

(b) The co-ordination of macroeconomic policies which shall be carried out gradually and in parallel with the programmes for the reduction of tariffs and the elimination of non-tariff restrictions referred to in the preceding paragraph;

(c) A common external tariff which encourages the foreign competitiveness of the States Parties;

(d) The adoption of sectoral agreements in order to optimize the use and mobility of factors of production and to achieve efficient scales of operation.

Article 6

The States parties recognize certain differentials in the rate at which the Republic of Paraguay and the Eastern Republic of Uruguay will make the transition. These differentials are indicated in the trade liberalization programme (Annex 1). Article 7

In the area of taxes, charges and other internal duties, products originating in the territory of one State Party shall enjoy, in the other States Parties, the same treatment as domestically produced products.

Article 8

The States Parties undertake to abide by commitments made prior to the date of signing of this Treaty, including agreements signed in the framework of the Latin American Integration Association (ALADI), and to co-ordinate their positions in any external trade negotiations they may undertake during the transitional period. To that end:

(a) They shall avoid affecting the interests of the States Parties in any trade negotiations they may conduct among themselves up to 31 December 1994; (b) They shall avoid affecting the interests of the other States Parties or the aims of the common market in any agreements they may conclude with other countries members of the Latin American Integration Association during the transition period;

(c) They shall consult among themselves whenever negotiating comprehensive tariff reduction schemes for the formation of free trade areas with other countries members of the Latin American Integration Association;

(d) They shall extend automatically to the other States Parties any advantage, favour, exemption, immunity or privilege granted to a product originating in or destined for third countries which are not members of the Latin American Integration Association. 


\title{
Appendix II Protocol of Ouro Preto Regarding the Institutional Structure of MERCOSUR
}

\section{(December 17, 1994)}

\author{
SUPPLEMENTARY PROTOCOL TO THE ASUNCION AGREEMENT ON THE \\ INSTITUTIONAL STRUCTURE OF THE MERCOSUR
}

-OURO PRETO PROTOCOL — The Argentine Republic, the Federative Republic of Brazil, the Republic of Paraguay and the Oriental Republic of Uruguay, hereinafter referred to as the "Party States:” In compliance with Article 18 of the Treaty of Asunción of March 26, 1991; Considering the importance of the advances already achieved and the implementation of a joint customs union as a stage in the creation of a common market;

Reaffirming the principles and objectives of the Treaty of Asunción and mindful of the need for special considerations towards the lesser developed countries and regions within the Mercosur; Mindful of the dynamics implicit in the entire integration process and the consequent need to adapt the institutional structure of the Mercosur to changes that have taken place;

Recognizing the outstanding job performed by the existing administrative bodies during the transition period;

Agree:

Chapter I

Mercosur Structure

Article I

The institutional structure of Mercosur shall comprise the following administrative bodies:

I-The Common Market Council (“CMC”);

185

Appendix 11187

Il-The Common Market Group (“CMG”);

111-The Mercosur Commerce Commission ("MCC”);

IV-The Joint Parliamentary Commission (“JPC”);

V-The Socio-Economic Consultative Forum ("SECF”)

VI-The Administrative Secretariat of Mercosur (“ASM”)

Sole paragraph-Any auxiliary organs deemed necessary to attain the objectives of the integration process may be created under the terms of this Protocol.

Article 2

The Common Market Council, the Common Market Group and the Mercosur

Commerce Commission are intergovernmental organizations with decisionmaking

powers.

Section 1

The Common Market Council

Article 3

The Common Market Council is the highest administrative body of the Mercosur.

It is charged with political leadership of the integration process, and with making

348

those decisions necessary to ensure that the objectives established by the Asunción

Agreement are met and that the common market becomes fully established.

Article 4

The Common Market Council shall consist of the Ministers of Foreign Relations and Economics, or their equivalents, from each Party State. 
Article 5

The Common Market Council's Chairmanship shall rotate among the Party

States, in alphabetical order, for six-month terms.

Article 6

The Common Market Council shall meet as often as it deems necessary, but no

less than once every six months, with participation by the Presidents of each

Party State.

Article 7

The Ministers of Foreign Relations shall coordinate the meetings of the Common Market Council, and other Ministers or authorities at the cabinet level may be invited to participate.

Article 8

The following are functions and powers of the Common Market Council:

I-To ensure the implementation of the Treaty of Asunción, its Protocols and the agreements signed within its framework;

Il-To formulate policies and promote necessary actions that will constitute the common market;

Ill—lb assume the legal personality of Mercosur;

$\mathrm{tV}$ - To negotiate and sign agreements on behalf of Mercosur, with third countries, groups of countries and international bodies. Said functions may be delegated by express mandate to the Common Market Group under the conditions set by Article XIV, paragraph VII;

V-To rule on proposals submitted to it by the Common Market Group; VT-To organize the

Ministers' meetings and rule on agreements submitted to it from those meetings;

$\mathrm{Vu}$ - To create, modify or eliminate whatever administrative bodies it deems appropriate;

VIII - To clarify the contents and scope of its Decisions, when deemed appropriate;

IX-To nominate the Director of the Mercosur Administrative Secretariat; $\mathrm{X}$-To issue financial and budgetary Decisions;

XI-To approve the internal operating procedures of the Common Market 


\section{APPENDIX D: EXTRACT OF THE CORRIDOR'S PROTOCOL OF INTENTIONS}

\section{Protocol of Intentions Atlantic Corridor for Mercosur ANEX I}

1 . South America is unequally occupied in demographic and economic terms, an enormous inland space almost empty. involved by a coastal belt of demographic density much superior.

Particularly, the Atlantic coast of the continent harbors most part of M I RCOSUL'S economy and population.

In this coastal belt three sub regions are identified:

The north-northeast region of Brazil. with a population of about 50 millions, and a GNP (Gross National Product) close to 60 billion dollars.

The South-Southea.st brazilian coastal region, with a population of about 90 millions of inhabitants, and GNP close to400 million dollars.

The region of River Plate, (Great Buenos Aires), and Uruguay, with population close 1020 millions of inhabitants, and GNP close to 70 billion dollars.

2 . These regions, are distributed alongside the Atlantic coast of South America. through considerable distances. For example, the state of MaranhAo. north of Brazil, and Estuário do Prata, (River Plate), measure nearto $5000 \mathrm{Km}$.

Despite this fact, the transportation is made basically by truck. As a consequence. the costs are very high, turning diflicult the interchange among the several sub-regions, nearly obstructing, for instance, an effective integration of the north-northeast region with the economic block of

MERCOSUL

Obviously, the ports and the coastal navigation should constitute the main element of role among these regions. And also, each port should perform the active linkage of development pole in its own region of influence.

3 . Considering these facts as of crucial importance for the balanced development of the MERCOSUL, the group of entities who sign this document, decided to form an organization aiming at addressing these questions in an integrated torm, In this respect, they propose to undertake the following activities:

a- Promote the creation of a real "MERCOSUL Atlantic Corridor" integrated by ports and terminals connected among themselves with a regular shipping lines system.

b - Attract the private investment for ports, navigation, and for installations of support to the system.

c- The efficient integration of these ports, with their own regions of influence, through efficient railroads, highways and river networks.

d . Gradual reduction of the transportation costs, based on the concept "door to door", through the transference from the intra coastal highway network to the coastal navigation of huge volumes of cargo.

e- Optimization of economic exchanges among the different regions involved, in special between the extreme points northnortheast and River Plate.

f- The transformation of ports, into poles of regional economic development and promoting centers of commerce and investment for their regions ofinfluence

$\mathrm{g}$. The creation of port-industrial zones in the coast of South America, through the attraction of industrial projects which benefit from the presence of ports.

$\mathrm{h}$. The planning of ecological urban and social development, of the port zones, aiming the protection and recuperation of the environmental property, the balanced development of the local infra-structure and the planned incorporation of the population of the port cities to the process.

4- Aiming the opcrationalization of theses goals, the constitution of the following institutional mechanisms are intended: a - A consortium formed by the mentioned entities, associated to private companies and business federations, in special to those belonging to the zone of influence ofthe various ports.

b . A South American center of port development, formed by universities and research centers of the regions involved.

c- The consortium will be organized in the terms of a non profit private society. Its activity will be, mainly carried out through partnerships and associations with investors and companies willing to participate, taking as example the "modus operandi" of the operational consortium of the CENTER- EAST corridor.

$\mathrm{d}$. The South American Center of Port Development will have as objective the formation of personnel, training, technological development and rendering services in the different fields involved in the project.

5- Based on this fact, the undersigned entities, undertake through this document, the commitment to develop jointly, their best efforts with a view to implement these intentions.

Rio de Janeiro, 05 September. 1996 